\title{
Search for the Trilepton Signal of the Minimal Supergravity Model in DØ Run II
}

\author{
D I S S E R T A T I O N \\ der \\ Fakultät für Physik \\ der \\ Ludwig-Maximilians-Universität München
}

vorgelegt von

Meta Binder

geboren in

Schäßburg, Rumänien

München, den 24. Juni 2005 

Erstgutachterin: Prof. Dr. Dorothee Schaile Zweitgutachter: Prof. Dr. Martin Faessler

Tag der mündlichen Prüfung: 22.09.2005 

To my nieces and nephew:

Birgit

Stefanie

Johannes 

$\ll$ You are

never given a wish without also being given the power to make it true.

You may

have to work for it,

however. $\gg$

Richard Bach 



\begin{abstract}
A search for associated chargino neutralino pair production is performed in the trilepton decay channel $q \bar{q} \rightarrow \widetilde{\chi}_{1}^{ \pm} \widetilde{\chi}_{2}^{0} \rightarrow \ell^{ \pm} \vee \widetilde{\chi}_{1}^{0} \mu^{ \pm} \mu^{\mp} \widetilde{\chi}_{1}^{0}$, using data collected with the D $\varnothing$ detector at a center-of-mass energy of $1.96 \mathrm{TeV}$ at the Fermilab Tevatron Collider. The data sample corresponds to an integrated luminosity of $\sim 300 \mathrm{pb}^{-1}$.

A dedicated event selection is applied to all samples including the data sample and the Monte Carlo simulated samples for the Standard Model background and the Supersymmetry signal. Events with two muons plus an additional isolated track, replacing the requirement of a third charged lepton in the event, are analyzed. Additionally, selected events must have a large amount of missing transverse energy due to the neutrino and the two $\tilde{\chi}_{1}^{0}$.

After all selection cuts are applied, 2 data events are found, with an expected number of background events of $1.75 \pm 0.34$ (stat.) \pm 0.46 (syst.). No evidence for Supersymmetry is found and limits on the production cross section times leptonic branching fraction are set.

When the presented analysis is considered in combination with three other decay channels, no evidence for Supersymmetry is found. Limits on the production cross section times leptonic branching fraction are set. A lower chargino mass limit of $117 \mathrm{GeV}$ at $95 \% \mathrm{CL}$ is then derived for the mSUGRA model in a region of parameter space with enhanced leptonic branching fractions.
\end{abstract}





\section{Zusammenfassung}

Eine Suche nach assoziierter Chargino-Neutralino-Paarproduktion wird im Trilepton Zerfallskanal $q \bar{q} \rightarrow \widetilde{\chi}_{1}^{ \pm} \widetilde{\chi}_{2}^{0} \rightarrow \ell^{ \pm} \vee \widetilde{\chi}_{1}^{0} \mu^{ \pm} \mu^{\mp} \widetilde{\chi}_{1}^{0}$ durchgeführt. Es werden Daten verwendet, die mit dem D $\varnothing$ Detektor bei einer Schwerpunktsenergie von $1.96 \mathrm{TeV}$ am Tevatron Beschleuniger am Fermilab gesammelt wurden.

Eine optimierte Selektion wird für alle Ereignismengen angewandt, einschließlich der Daten und Monte Carlo Datensätze für Standardmodelluntergrund und Supersymmetriesignal. Ereignisse mit zwei Myonen und einer zusätzlichen isolierten Spur, anstatt der Identifikation eines dritten geladenen Leptons, werden selektiert. Selektierte Ereignisse haben zusätzlich einen großen Betrag an fehlender transversaler Energie aufgrund des Neutrinos und der beiden $\widetilde{\chi}_{1}^{0}$.

Nach allen Selektionsschnitten, werden 2 Datenereignisse gefunden und ein Untergrund von $1.75 \pm 0.34$ (stat.) \pm 0.46 (syst.) Ereignissen erwartet. Kein Hinweis auf die Existenz von Supersymmetrie wird gefunden. Daher wird eine Schranke für den Produktionswirkungsquerschnitt und das leptonische Verzweigungsverhältnis berechnet.

Wird die vorliegende Analyse mit drei anderen Zerfallskanälen kombiniert, ergibt sich auch kein Hinweis auf die Existenz von Supersymmetrie. Daher werden Schranken an den Produktionswirkungsquerschnitt und das leptonische Verzweigungsverhältnis gesetzt. Eine untere Schranke für die Charginomasse von $117 \mathrm{GeV}$ bei $95 \% \mathrm{CL}$ wird für das mSUGRA Modell bestimmt, in einem Parameterraum mit erhöhten leptonischen Verzweigungsverhältnissen. 



\section{Contents}

1 Introduction 1

2 Theoretical Background $\quad 3$

2.1 The Standard Model . . . . . . . . . . . . . . . . . . . . . 3

2.1.1 Limitations of the Standard Model . . . . . . . . . . . 5

2.2 Supersymmetry ........................ 6

2.3 The Minimal Supersymmetric Standard Model . . . . . . . . . . . . . 8

2.3.1 R-Parity ..................... 8

2.3.2 Mass Spectrum . . . . . . . . . . . . . . . . 99 9

2.3.3 Soft Supersymmetry Breaking . . . . . . . . . . . . . . 9

2.4 The Minimal Supergravity Model (mSUGRA) . . . . . . . . . . . . . . . 11

2.4.1 Supersymmetry and Electroweak Breaking . . . . . . . . . . . 11

2.4 .2 Parameters of mSUGRA . . . . . . . . . . . . . 12

2.4.3 Constraints of the mSUGRA Model . . . . . . . . . . . . . 13

2.5 Phenomenology of mSUGRA . . . . . . . . . . . . . . . . . 14

2.5.1 Squarks and Sleptons . . . . . . . . . . . . . . . . . . 14

2.5.2 Gauginos, Higgsinos and the Higgs Bosons . . . . . . . . . . . 16

2.5.3 Creation and Decay of Supersymmetric Particles . . . . . . . . 18

2.5.3.1 Decays of Squarks and Sleptons . . . . . . . . . . . 18

2.5.3.2 Decays of Neutralinos and Charginos . . . . . . . 19

2.5.4 The Trilepton Signature at the Tevatron . . . . . . . . . . . 20

2.5.4.1 Cross Section . . . . . . . . . . . 21

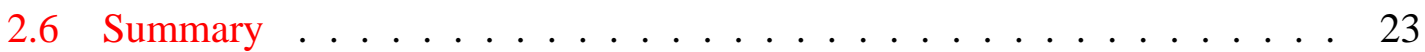

3 Experimental Overview $\mathbf{2 5}$

3.1 Fermilab Accelerator Chain . . . . . . . . . . . . . . . . . . . 26

3.2 DØ Detector . . . . . . . . . . . . . . . . . . 28

3.2.1 Tracking Detectors . . . . . . . . . . . . . 30

3.2 .2 Calorimeter ........................ 32

3.2 .3 Muon System . . . . . . . . . . . . . . . . . . . . . . . 33

3.2.4 Trigger and Data Acquisition System . . . . . . . . . . . . . . . 35

3.2 .5 Muon Trigger . . . . . . . . . . . . . . . . . . . 37

3.2 .6 Luminosity Monitor . . . . . . . . . . . . . . . . 38 
4 Monte Carlo and Data Samples 39

4.1 Event Simulation . . . . . . . . . . . . . . . . . . . . . 39

4.2 Monte Carlo Samples . . . . . . . . . . . . . . . . . . . . 40

4.2 .1 SUSY Signals . . . . . . . . . . . . . . . . . . . . . . . . . . . . . . .

4.2.2 Standard Model Backgrounds . . . . . . . . . . . . . . 42

4.3 Data Samples . . . . . . . . . . . . . . . . . . . . . 43

4.3.1 Trigger Selection and Efficiency . . . . . . . . . . . . . . . 44

4.3.2 Integrated Luminosity _ . . . . . . . . . . . . . . . . 44

4.4 Monte Carlo Normalization to Data . . . . . . . . . . . . . . . . 45

4.5 Event Reconstruction and Physics Objects . . . . . . . . . . . . . . . . . 46

4.5.1 Muon Particles: Identification and Efficiency . . . . . . . . . . . 47

4.5.2 Muon Momentum Smearing for Monte Carlo Samples . . . . . . 49

4.5.3 Jets and Missing Transverse Energy $\left(\mathbb{E}_{T}\right) \ldots \ldots \ldots$

4.6 QCD Background Estimation . . . . . . . . . . . . . . 52

5 Trilepton Analysis $\quad \mathbf{5 7}$

5.1 Motivation of Selection Cuts . . . . . . . . . . . . . . 57

5.2 Event Selection . . . . . . . . . . . . . . . . . 62

5.2.1 Muon Transverse Momenta: $p_{T}^{\mu_{1}}, p_{T}^{\mu_{2}} \ldots \ldots$. . . . . . . 63

5.2.2 Invariant Dimuon Mass: $M_{\mu \mu} \ldots \ldots \ldots$. . . . . . . . . 64

5.2.3 Missing Transverse Energy: $\mathbb{E}_{T} \ldots \ldots \ldots 6$

5.2 .4 Additional Isolated Track: $p_{T}^{t r} \ldots \ldots . \ldots . . \ldots 67$

5.2.5 Invariant Mass: $M_{\mu t r} \ldots \ldots \ldots$. . . . . . . . . . 69

5.2.6 $p_{T}$-Balance: $\Sigma_{p_{T}} / p_{T}^{t r} \ldots \ldots \ldots \ldots \ldots$

5.2.7 Transverse Mass $M_{T}$ and $\operatorname{Sig}\left(\mathbb{E}_{T}\right): \ldots \ldots \ldots 71$

5.2.8 Combination of $\mathbb{E}_{T}$ and Track: $p_{T}^{t r} \times \mathbb{E}_{T} \ldots \ldots \ldots 73$

5.3 Comparison of Data and Background Events . . . . . . . . . . . . . 75

5.4 SUSY Points: Chargino and Slepton Mass Scan . . . . . . . . . . . . . . 77

5.5 Systematic Uncertainties . . . . . . . . . . . . . . . . 81

6 Results $\quad \mathbf{8 5}$

6.1 Limit for $\sigma \times B R(3 \ell) \ldots \ldots \ldots \ldots$. . . . . . . . . . . . . . . . . . . 85

6.2 Event Displays of the Two Candidate Events . . . . . . . . . . . . . . . . . 89

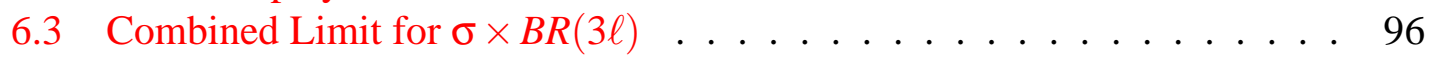

7 Summary and Conclusions $\quad 99$

A The Compressed Data Format: ThumbNail 101

$\begin{array}{ll}\text { Bibliography } & 103\end{array}$

$\begin{array}{lr}\text { Acknowledgements } & 108\end{array}$

$\begin{array}{lr}\text { Curriculum Vitae } & \mathbf{1 1 0}\end{array}$ 


\section{Chapter 1}

\section{Introduction}

The questions concerning the origin of our universe have long been thought of as metaphysical and outside the realm of physics. But tremendous progress in experimental techniques allows us to study the universe nowadays. The large scale structures of the universe are investigated with space telescopes. The tiniest building blocks of matter - the quarks and leptons - are analyzed with large accelerators. The results obtained from both areas of active research provide a better understanding of the creation and evolution of our universe.

A corner stone in this field is Elementary Particle Physics which is the study of the building blocks of matter and the interactions between them. These particles, the quarks and leptons, can be grouped according to certain symmetry principles and their interactions. The theory of the Standard Model describes these particles and their interactions remarkably successful. But it also contains a variety of questions which are left unanswered and can not be explained within the Standard Model itself. For example, the origin of electroweak symmetry breaking is not known and the origin of dark matter, which seems to provide the majority of mass in our universe, is not described by the Standard Model. So it seems, that the Standard Model will have to be extended.

Do we really know what lies beyond the Standard Model? How do we know what we will find at higher energies? Even if we don't know what physics to expect at higher energies, the principles of physics in the explication of the Standard Model should apply within a new theory and in addition, some of the open questions should be answered. The theory of Supersymmetry [1] is such a theory.

Supersymmetry is a novel type of symmetry. It relates the properties of the bosons to those of the fermions. Its validity in physics follows from the common belief in unification. There is no Grand Unified Theory without Supersymmetry. In such theories the electromagnetic, weak and strong force are unified. Although such a single unified force cannot be observed directly, there are several predictions which can be verified at low energies.

The Minimal Supersymmetric Extension of the Standard Model is a Grand Unified Theory. It predicts many unrelated phenomena. Among them: the unification of coupling constants, the existence of dark matter and the electroweak symmetry breaking at a scale 
far below the unification scale. The predictions of this model can be probed at today's accelerators.

Supersymmetry is a theory with a spontaneously broken symmetry. The mechanism of Supersymmetry breaking is mediated to the particles of the Minimal Supersymmetric Standard Model via gravitational interactions. The underlying theory is called minimal Supergravity and is minimal to that extent that only five parameters are needed to describe the supersymmetric particles and their interactions. The choice of these parameters at an high energy scale, defines the production and decay of the supersymmetric particles at low energy scales. This dissertation focuses on the predictions of minimal Supergravity at an energy scale of $1 \mathrm{TeV}^{1}$ at the Fermilab Tevatron Collider.

The theoretical background is presented in chapter 2, where first a very brief description of the Standard Model (section 2.1) is given, followed by a motivation for Supersymmetry (section 2.2) and a short discription of the Minimal Supersymmetric Standard Model (section 2.3). The theory of minimal Supergravity is presented section 2.4. The phenomenology of the minimal Supergravity model is given in section 2.5, emphasizing the properties of the supersymmetric signatures under investigation in this dissertation.

A particularly promising process to search for Supersymmetry at the Fermilab Tevatron Collider is the trilepton signal. The data events are collected with the DØ detector. The experimental overview of the Tevatron Collider and the D $\varnothing$ detector, is given in chapter 3. The data sample is compared with the Monte Carlo simulated samples of the Standard Model background and the trilepton signal. The simulation and reconstruction of events is described in chapter 4.

The trilepton signal has a clean signature in the detector, with no hadronic activity expected in the event. It is a multi-lepton final state, together with the characteristic missing transverse momentum from the lightest supersymmetric particles (LSPs) which are stable and escape detection. The Standard Model sources with comparable event toplogies are reliably predictable. The search strategies and the actual event selection is presented in chapter 5 . The obtained results are discussed in chapter 6 followed by a summary and conclusions in chapter 7 .

The presented dissertation performs a test of the theory of Supersymmetry, which is known as a theory "Beyond the Standard Model". Although the mass scale of Supersymmetry depends on many parameters, there is good reason to expect the observation of supersymmetric particles at the center-of-mass energy provided by the Tevatron. There, the trilepton events may be the most promising channel to look for Supersymmetry.

\footnotetext{
${ }^{1}$ In this dissertation the convention $\mathrm{c}=\hbar=1$ is always assumed.
} 


\section{Chapter 2}

\section{Theoretical Background}

\subsection{The Standard Model}

The Standard Model of the electromagnetic, weak and strong interaction [2, 3, 4] is the foundation of the current understanding of all elementary particles. It describes all forces, but gravitation. Half-integer spin fermions act as the building blocks of matter with integer spin bosons acting as mediators of forces. Table 2.1 lists the fermions together with the generation they belong to and the relevant quantum numbers. The fermions are grouped

\begin{tabular}{|c|c|c|c|c|c|c|c|c|c|}
\hline \multirow[t]{2}{*}{ Fermions } & \multicolumn{3}{|c|}{ Generations } & \multirow{2}{*}{$\begin{array}{r}\text { Charge } \\
\text { Q }\end{array}$} & \multirow{2}{*}{$\begin{array}{r}\text { Weak } \\
\text { isospin }\end{array}$} & \multirow{2}{*}{$\begin{array}{l}\text { Color } \\
\text { charge }\end{array}$} & \multirow{2}{*}{$\begin{array}{c}\text { Lepton } \\
\text { number } \\
\text { L }\end{array}$} & \multirow{2}{*}{$\begin{array}{c}\text { Baryon } \\
\text { number } \\
\text { B }\end{array}$} & \multirow[t]{2}{*}{ Spin } \\
\hline & $1 \mathrm{st}$ & 2nd & $3 \mathrm{rd}$ & & & & & & \\
\hline \multirow[t]{2}{*}{ Leptons } & $v_{e}$ & $v_{\mu}$ & $v_{\tau}$ & 0 & $+\frac{1}{2}$ & 0 & 1 & 0 & $\frac{1}{2}$ \\
\hline & $e^{-}$ & $\mu^{-}$ & $\tau^{-}$ & -1 & $-\frac{1}{2}$ & 0 & 1 & 0 & $\frac{1}{2}$ \\
\hline \multirow[t]{2}{*}{ Quarks } & $\mathrm{u}$ & $\mathrm{c}$ & $\mathrm{t}$ & $+\frac{2}{3}$ & $+\frac{1}{2}$ & $\mathrm{r}, \mathrm{g}$ or $\mathrm{b}$ & 0 & $\frac{1}{3}$ & $\frac{1}{2}$ \\
\hline & $\mathrm{d}$ & $\mathrm{s}$ & $\mathrm{b}$ & $-\frac{1}{3}$ & $-\frac{1}{2}$ & $\mathrm{r}, \mathrm{g}$ or $\mathrm{b}$ & 0 & $\frac{1}{3}$ & $\frac{1}{2}$ \\
\hline Bosons & \multicolumn{3}{|c|}{ Coupling } & $\begin{array}{c}\text { Charge } \\
\text { Q }\end{array}$ & $\begin{array}{r}\text { Weak } \\
\text { isospin }\end{array}$ & $\begin{array}{l}\text { Color } \\
\text { charge }\end{array}$ & $\begin{array}{c}\text { Lepton } \\
\text { number } \\
\text { L }\end{array}$ & $\begin{array}{c}\text { Baryon } \\
\text { number } \\
\text { B }\end{array}$ & Spin \\
\hline Photon & Ele & nag. & $\gamma$ & 0 & 0 & 0 & 0 & 0 & 1 \\
\hline \multirow[t]{3}{*}{ Weakons } & \multirow{3}{*}{\multicolumn{2}{|c|}{ Weak }} & $W^{+}$ & +1 & +1 & 0 & 0 & 0 & 1 \\
\hline & & & $Z$ & 0 & 0 & 0 & 0 & 0 & 1 \\
\hline & & & $W^{-}$ & -1 & -1 & 0 & 0 & 0 & 1 \\
\hline Gluons & \multicolumn{2}{|c|}{ Strong } & G & 0 & 0 & $\begin{array}{l}1 \text { color }+ \\
1 \text { anti-color }\end{array}$ & 0 & 0 & 1 \\
\hline
\end{tabular}

Table 2.1: Elementary fermions and bosons, known in the Standard Model, and their assigned quantum numbers. Anti-fermions, which are not listed, have inverted numbers of the signs of charge, weak isospin, color charge, lepton and baryon number. 
in two categories according to their interactions: the quarks and the leptons. Leptons can be found as free particles in nature, whereas the quarks appear always in a confined state within hadrons.

The strong and the electroweak interactions between the particles of the Standard Model are mediated by gauge bosons: eight massless gluons for the strong interaction, the massive bosons $Z, W^{ \pm}$and the massless photon $\gamma$ for the electroweak interaction. Table 2.1 also lists the bosons indicating the relevant quantum numbers. The quantum numbers are conserved in the interactions between the particles. For each fermionic particle in the Standard Model, there is an anti-particle, with the same mass, the same spin and lifetime but with opposite quantum numbers of charge, weak isospin, color charge, lepton number and baryon number. Additionally the fermions and anti-fermions are characterized by their chirality or handedness, i.e. they can be left- or right-handed. The neutrinos are only left-handed and the anti-neutrinos are only right-handed particles. In this description of the Standard Model neutrinos are considered massless.

The Standard Model is based on the gauge principle according to which all the forces of Nature are mediated by an exchange of the massless gauge fields of the corresponding local symmetry group [4]. The gauge symmetry group of the Standard Model is

$$
\mathrm{SU}(3)_{\text {color }} \times \mathrm{SU}(2)_{\text {left }} \times \mathrm{U}(1)_{\mathrm{Y}}
$$

where $\mathrm{SU}(3)_{\text {color }}$ is the color group, $\mathrm{SU}(2)_{\text {left }}$ is the weak isospin group and $\mathrm{U}(1)_{\mathrm{Y}}$ is the group of weak hypercharge ${ }^{1}$. The strengths of the forces are determined by the associated coupling constants for each group $g_{s}, g$, and $g^{\prime}$ respectively.

The associated bosons of the electroweak gauge symmetry group $\operatorname{are}^{2} W^{i}, i=1,2,3$ and $B$. They become massive via spontaneous breaking of the $\mathrm{SU}(2)_{\text {left }} \times \mathrm{U}(1)_{\mathrm{Y}}$ gauge symmetry into the remaining $\mathrm{U}(1)$ em gauge symmetry of the electromagnetic interaction. This spontaneous symmetry breaking is achieved via the Higgs mechanism: when the scalar Higgs field (which is introduced into the theory) acquires a non-zero vacuum expectation value, the electroweak gauge symmetry is spontaneously broken and three gauge bosons become massive. The mass eigenstates of the gauge bosons are three massive gauge bosons, the $Z, W^{ \pm}$and one massless gauge boson the photon $\gamma$. As a consequence of the Higgs mechanism, a scalar spin-0 neutral particle appears in the theory associated with the electroweak field, the Higgs boson. It has not been discovered at the time of this writing. The Higgs boson couples to all quarks and charged leptons via Yukawa coupling, which permits them to acquire mass, while the photon and the gluons remain massless.

Next to the strong, weak and electromagnetic force described in the Standard Model, there is the gravitational force. Its mediator is the graviton, a spin-2 particle, which has not been discovered yet.

\footnotetext{
${ }^{1}$ The weak hyperchage is defined by the Gell-Mann-Nishijima equation as $Q=T_{3}+\frac{Y}{2}$, where $T_{3}$ is the third component of the weak isospin and $\mathrm{Q}$ is the electric charge.

${ }^{2}$ Lorentz indices are suppressed for simplicity
} 


\subsubsection{Limitations of the Standard Model}

The Standard Model has been tested up to a very high precision. The accuracy of its predictions corresponds to experimental data mostly even much better than one percent [5]. All Standard Model particles, except for the Higgs boson, have been discovered experimentally. Nevertheless the Standard Model leaves some open questions, some of which are discussed below. They should be answered within a new theory of elementary particle physics.

- The Standard Model depends on at least 19 arbitrary parameters whose values are chosen to fit the experimental data: the values of the electromagnetic, the weak and the strong coupling constant, the mass of the six quarks and the three massive leptons, the four parameters of the Cabbibo-Kobayashi-Maskawa matrix ${ }^{3}$, the mass of one of the electroweak bosons $\left(W^{ \pm}\right.$or $Z$ ), the Higgs boson mass $m_{H}$ and the parameter $\theta$ for the strong $C P$ violation.

- The mechanism of the electroweak symmetry breaking is still unclear. In the Standard Model it is accomplished in an ad hoc fashion by the introduction of a scalar Higgs field in the theory. In nature the symmetry breaking may well be realized in a different manner.

- In Grand Unified Theories (GUTs) [6] the known electromagnetic, weak and strong forces are combined into a single theory. At the energy scale of $M_{G U T} \sim 10^{16} \mathrm{GeV}$ these forces are equally strong. The difference between the weak energy scale $M_{W} \sim 10^{2} \mathrm{GeV}$ and the GUT energy scale is huge. This is already a powerful clue for physics beyond the Standard Model and is known as the hierarchy problem.

- The Standard Model Higgs field requires a non-vanishing vacuum expectation value at the minimum of its potential. From measurements of the properties of weak interactions, $m_{H}^{2}$ is roughly of the order of $(100 \mathrm{GeV})^{2}$. However, $m_{H}^{2}$ receives large quantum corrections from the virtual effects of every particle which couples to the Higgs field. When the Standard Model is embedded into a larger theory involving a high-energy scale, an ultraviolet momentum cutoff $\Lambda_{U V}$ is used to regulate these loop integrals. The quantum corrections to $m_{H}^{2}$ are in the order of $\Lambda_{U V}^{2}$. If $\Lambda_{U V}$ is of the order of the Planck scale ${ }^{4} M_{P}=1.2 \cdot 10^{19} \mathrm{GeV}$ [5], the quantum corrections to $m_{H}^{2}$ are some 30 orders of magnitude larger than the aimed-for value. The quantum corrections to the Higgs mass need an incredible fine-tuning in the Standard Model to cancel the quadratic divergences [7].

- The couplings of the Standard Model are not constant, but a function of the energy. The strong $g_{s}$ and weak coupling $g$ decrease with energy, while $g^{\prime}$ on the contrary

\footnotetext{
${ }^{3}$ The Cabbibo-Kobayashi-Maskawa matrix relates the quark mass eigenstates and the weak eigenstates. The four parameters are three mixing angles and one phase. [5]

${ }^{4}$ This is the energy scale where the strength of gravitational interactions of elementary particles become comparable to their gauge interactions (electroweak and strong).
} 
increases, so it is possible that they become equal at some energy scale. When extrapolating the measured couplings up to a high energy scale, the unification at a single point is not possible within the Standard Model.

\subsection{Supersymmetry}

There are various theories that extend the Standard Model. Supersymmetry is one option for new physics on a low-energy scale. It is considered in further detail in this dissertation.

Supersymmetry introduces a new symmetry between bosons and fermions: the number of bosonic degrees of freedom equals the number of fermionic degrees of freedom. In such a theory all particles and their interactions can be described in one symmetry group $[8,9,10]$.

A Supersymmetry transformation changes a fermionic state into a bosonic state and vice versa. The resulting new particles are called supersymmetric particles (sparticles). The supersymmetric bosons and fermions are also referred to as superpartners of their Standard Model partners. The bosonic superpartner of a fermion is called sfermion, and the fermionic superpartner of a gauge boson is called gaugino. The superpartners differ by spin 1/2 from their Standard Model partners, while all other quantum numbers are equal, also the masses should be equal. The absence of such sparticles explains why the Supersymmetry must be broken and implicates a Supersymmetry breaking by which superpartners gain masses much larger than their Standard Model partners.

Supersymmetry provides a promising ingredient to the unification of particle physics and gravity, which is governed by the Planck scale. The generators of Supersymmetry create the following chain of states:

$$
\operatorname{spin} 2 \rightarrow \operatorname{spin} 3 / 2 \rightarrow \operatorname{spin} 1 \rightarrow \operatorname{spin} 1 / 2 \rightarrow \operatorname{spin} 0
$$

The Supersymmetry algebra solves the problem to unify all four forces of Nature in one algebra (spin-1 gauge bosons and spin-2 graviton) [11].

Supersymmetry legitimizes the introduction of Higgs scalar fields, because it connects spin-0 and spin-1/2 fields. Therefore it naturally gives rise to the mechanism of electroweak symmetry breaking.

Supersymmetry offers a very attractive cure for the fine-tuning problem. The Higgs fields have additional interactions involving for example squark loops which also produce a quadratic divergence, canceling the quadratic divergence from the quark loops (see section 2.1.1). Therefore it preserves the given hierarchy of two energy scales $\left(M_{W}, M_{P}\right)$. This is why Supersymmetry is said to solve the hierarchy problem.

An important motivation for Supersymmetry is related with the Grand Unified Theories wherein the gauge symmetry increases with energy. Neglecting gravity for the time being due to its weakness, the idea of the GUT is the following: the fundamental interactions (strong, weak, electromagnetic) are different branches of a unique interaction associated with a gauge group. This unifying gauge group is broken at a high-energy 
scale $\left(10^{16} \mathrm{GeV}\right)$ and the unique interaction is split into three branches: strong, weak and electromagnetic interaction.

The evolution of the associated coupling constants is described by the renormalization group (RG) equations and is confirmed experimentally. The three coupling constants can be written as $\alpha_{1}=(5 / 3) g^{2} /(4 \pi)=5 \alpha /\left(3 \cos ^{2} \theta_{W}\right), \alpha_{2}=g^{2} /(4 \pi)=\alpha / \sin ^{2} \theta_{W}$ and $\alpha_{3}=g_{s}^{2} /(4 \pi)$, where $\theta_{W}$ the weak mixing angle, $\alpha$ is the fine structure constant and $g_{s}, g, g^{\prime}$ are the usual coupling constants of the Standard Model [11]. In the one-loop approximation the reciprocals of the coupling constants $\alpha_{i}^{-1}, i=1,2,3$ run linearly with the logarithm of the RG equation scale (fig. 2.1).
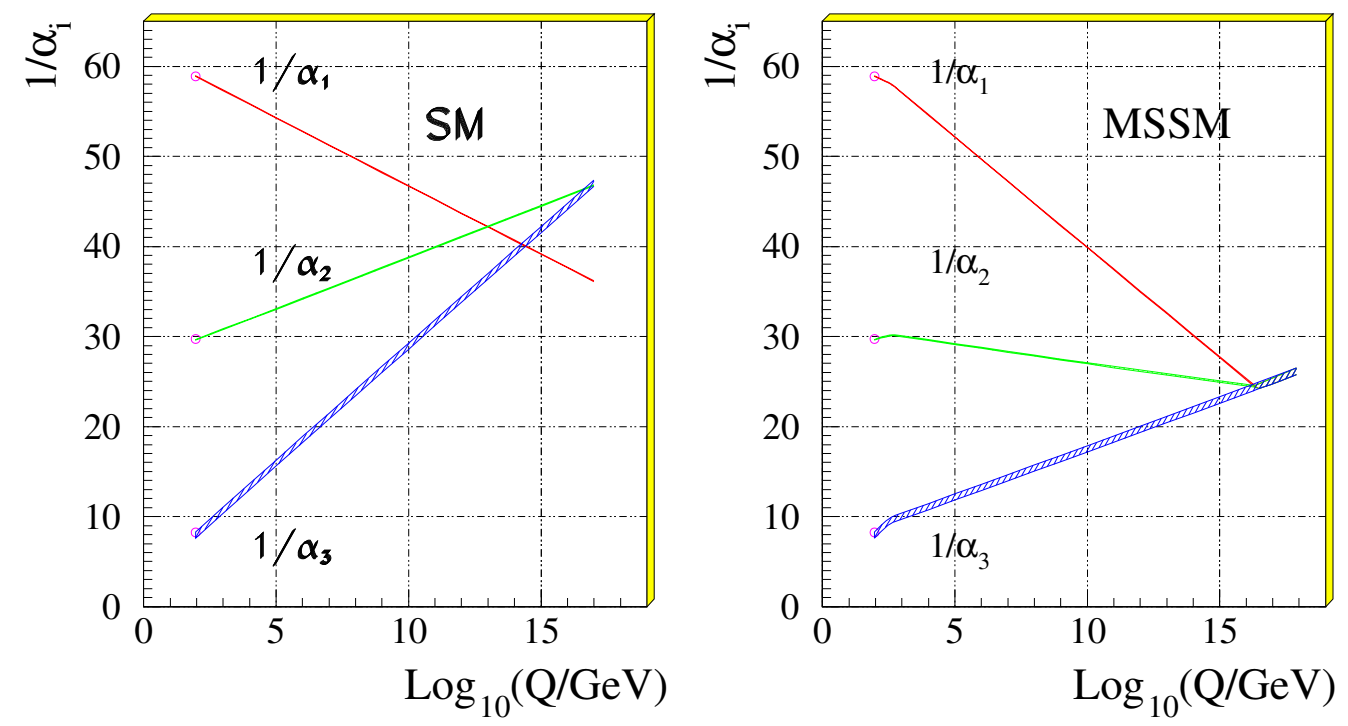

Figure 2.1: Evolution of the inverse of the coupling constants $\alpha_{i}^{-1}(\mathrm{Q})$ in the Standard Model (left) and the MSSM (right) as a function of the energy Q. The evolution of the couplings shows the first order loop corrections. The second order corrections are small and do not cause any visible deviation from the straight lines shown [11].

A unification of the gauge couplings within the Standard Model is not possible. The gauge couplings unify remarkably well in the $\mathrm{MSSM}^{5}$, when supersymmetric particles contribute effectively to the running of the coupling constants, which changes the slope of the lines near $1 \mathrm{TeV}$. The unification of the three curves at one single point is not trivial. When introducing new particles all three curves are influenced simultaneously, thus giving rise to strong correlations between the slopes of the three lines.

The apparent unification of the gauge couplings at a value $\sim 10^{16} \mathrm{GeV}$ could be just an accident or a strong hint in favor of a GUT theory, which indeed predicts gauge unification below $M_{P}$. Furthermore, a similar RG equation analysis can be applied to the couplings and masses of the MSSM [7], see for example figure 2.3. Nevertheless, unification does not prove Supersymmetry. The real proof would be the observation of the sparticles.

\footnotetext{
${ }^{5}$ The Minimal Supersymmetric Standard Model (MSSM) is described in section 2.3.
} 


\subsection{The Minimal Supersymmetric Standard Model}

In every supersymmetric extension of the Standard Model the characteristic feature is the presence of the supersymmetric particles. The supersymmetric structure of such a theory must include the known particles, their superpartners and the interactions between them. It is then possible to evaluate the experimental consequences of Supersymmetry at the weak energy scale $M_{W}[11,12,13]$.

The simplest possibility for such a theory is a supersymmetric extension of the Standard Model - the Minimal Supersymmetric Standard Model (MSSM). The MSSM is minimal in the sense that it contains the smallest number of new particles and new interactions necessary to be compatible with the phenomenology of Supersymmetry.

The field content of the MSSM with the $\mathrm{SU}(3)_{\text {color }} \times \mathrm{SU}(2)_{\text {left }} \times \mathrm{U}(1)_{\mathrm{Y}}$ gauge invariance, consists of the three generations of quarks plus the gauge bosons of the Standard Model, and all their superpartners (sfermions and gauginos). Higgs fields cannot be superpartners of quarks and leptons, as this would induce spontaneous violation of baryon and lepton number conservation. In addition the single complex Higgs doublet of the Standard Model is replaced with two complex chiral Higgs multiplets to give masses to up- and down-type quarks (and charged leptons). The superpartners of the Higgs particles are the higgsinos. The graviton is included in the MSSM and its superpartner is the gravitino [7].

\subsubsection{R-Parity}

All supersymmetric interactions consistent with baryon and lepton number conservation ( $B-L$ conservation) are included in the MSSM. Since the MSSM does not include $B-L$ violating interactions, it possess a multiplicative R-parity invariance for a particle with spin $S$ defined as [14]

$$
R=(-1)^{3(B-L)+2 S}
$$

The multiplicative quantum number $R$ is conserved and distinguishes the Standard Model particles with $R=+1$ from their superpartners with $R=-1$.

The conservation of R-parity in scattering and decay processes predicts that the interactions of superpartners are the same as in the Standard Model and has a deep impact on supersymmetric phenomenology. For example, the supersymmetric particles are only produced in pairs from an initial state of "standard" particles. The sparticles are in general highly unstable and decay into lighter states. At the end of this decay chain is the lightest supersymmetric particle (LSP).

If R-parity invariance exists, then the LSP is stable as it is the lightest and cannot decay into other supersymmetric particles. Furthermore the LSP is weakly-interacting with ordinary matter, i.e. its behavior is similar to a heavy stable neutrino. Moreover a stable LSP is a prime candidate for dark matter that is required in many models of cosmology and galaxy formation [15]. 


\subsubsection{Mass Spectrum}

The mass eigenstates of the particles of the MSSM (the sparticles and their associated Standard Model partners) are listed in table 2.2 The spin and R-parity quantum numbers of all particles are indicated.

\begin{tabular}{|c|c|c|c|c|c|c|c|}
\hline Particles & & Spin & $R$ & Sparticles & & Spin & $R$ \\
\hline left-handed quarks & $q_{L}$ & $1 / 2$ & +1 & "left" squarks & $\widetilde{q}_{L}$ & 0 & -1 \\
\hline right-handed quarks & $q_{R}$ & $1 / 2$ & +1 & "right" squarks & $\widetilde{q}_{R}$ & 0 & -1 \\
\hline left-handed leptons & $l_{L}$ & $1 / 2$ & +1 & "left" sleptons & $\tilde{\ell}_{L}$ & 0 & -1 \\
\hline right-handed leptons & $l_{R}$ & $1 / 2$ & +1 & "right" sleptons & $\tilde{\ell}_{R}$ & 0 & -1 \\
\hline gluons & $g$ & 1 & +1 & gluinos & $\tilde{g}$ & $1 / 2$ & -1 \\
\hline weak charged boson & $W^{ \pm}$ & 1 & +1 & & & & \\
\hline charged Higgs & $H^{ \pm}$ & 0 & +1 & charginos & $\tilde{\chi}_{1,2}^{ \pm}$ & $1 / 2$ & -1 \\
\hline photon & $\gamma$ & 1 & +1 & & & & \\
\hline weak neutral boson & $Z^{0}$ & 1 & +1 & & & & \\
\hline neutral Higgs & $h^{0}, H^{0}, A^{0}$ & 0 & +1 & neutralinos & $\tilde{\chi}_{1,2,3,4}^{0}$ & $1 / 2$ & -1 \\
\hline graviton & & 2 & +1 & gravitino & & $3 / 2$ & -1 \\
\hline
\end{tabular}

Table 2.2: The mass eigenstates of the particles of the MSSM. The index $L, R$ of the sleptons and squarks refers to the chirality of their Standard Model partners.

All left-handed and all right-handed quarks as well as their superpartners appear in three possible color states $(\mathrm{r}, \mathrm{b}, \mathrm{g})$. The interactions of the squarks and sleptons are the same as for the corresponding Standard Model fermions. From the eight degrees of freedom in the two Higgs doublets three are absorbed to give rise to the $W^{ \pm}$and the $Z$ masses (known from the Standard Model) leaving five physical Higgs bosons. Those are the two charged $\left(H^{ \pm}\right)$and three neutral $\left(h^{0}, H^{0}, A^{0}\right)$ Higgs bosons of the MSSM.

The gauginos and higgsinos are massive and mix. The neutral gauginos and higgsinos mix to form the neutralinos $\left(\widetilde{\chi}_{i}^{0}, \mathrm{i}=1,2,3,4\right)$ while the charged higgsinos and gauginos mix to form the charginos $\left(\widetilde{\chi}_{i}^{ \pm}, \mathrm{i}=1,2\right)$.

\subsubsection{Soft Supersymmetry Breaking}

Supersymmetry, if it is realized in nature, cannot be an exact symmetry, as we do not see squarks nor sleptons which are mass degenerate with the quarks or leptons. Thus Supersymmetry must be a broken symmetry.

In the MSSM, Supersymmetry (SUSY) breaking is parameterized by adding Supersymmetry breaking terms by hand to the supersymmetric Lagrangian of the theory. The Lagrangian of the MSSM can then be written in the form [16]

$$
\mathcal{L}=\mathcal{L}_{\mathrm{SUSY}}+\mathcal{L}_{\text {soft }}
$$


where $\mathcal{L}_{\text {SUSY }}$ preserves Supersymmetry invariance and $\mathcal{L}_{\text {soft }}$ violates Supersymmetry but contains only masses and couplings which preserve the given hierarchy and do not disturb the cancellation of quadratic divergences. The terms in $\mathcal{L}_{\text {soft }}$ are called soft-breaking terms. This introduces many new parameters, which are not present in the ordinary Standard Model. The characteristic mass scale for the soft-breaking terms is around $1 \mathrm{TeV}$.

The parameters of the MSSM can be described by considering separately the Supersymmetry conserving and the Supersymmetry breaking sector. The Supersymmetry conserving sector contains the particles and couplings of the Standard Model:

- three gauge couplings $g_{s}, g, g^{\prime}$

- one Higgs mass parameter $\mu$

- Higgs-fermion Yukawa coupling constants corresponding to the coupling of quarks, leptons and their superpartners, to the Higgs bosons and higgsinos

The Supersymmetry breaking sector is characterized by the soft-breaking terms mentioned above:

- gaugino masses ( $M_{1}, M_{2}$ and $M_{3}$ for the $\mathrm{U}(1), \mathrm{SU}(2)$ and $\mathrm{SU}(3)$ gauge groups)

- mass terms for various left- and right- spin-0 fields (squark, slepton, Higgs)

- trilinear scalar couplings

- three scalar Higgs squared-mass parameters: two diagonal $\left(m_{H_{u}}^{2}, m_{H_{d}}^{2}\right)$ and one offdiagonal $(B$ term)

The vacuum expectation values for the Higgs fields are $\left\langle H_{u}\right\rangle=v_{u}$ and $\left\langle H_{d}\right\rangle=v_{d}$ at the minimum of the scalar potential of the MSSM. They can be connected with the mass of the $Z$ boson and the electroweak gauge couplings as $v_{u}^{2}+v_{d}^{2}=2 m_{Z}^{2} /\left(g^{2}+g^{2}\right)$. The ratio

$$
\tan \beta \equiv v_{u} / v_{d}
$$

is a free parameter of the model [7]. The requirement for electroweak symmetry breaking then requires

$$
\begin{aligned}
& m_{H_{d}}^{2}+|\mu|^{2}=B \tan \beta-\frac{1}{2} m_{Z}^{2} \cos 2 \beta \\
& m_{H_{u}}^{2}+|\mu|^{2}=B \cot \beta+\frac{1}{2} m_{Z}^{2} \cos 2 \beta .
\end{aligned}
$$

The two parameters $B$ and $|\mu|$ can be eliminated in favor of $\tan \beta$, leaving the sign of $\mu$ as a free parameter $[7,17]$.

The large number of soft-breaking terms of 106 such as masses, phases and mixing angles can be greatly reduced when the unification constraints at a high energy scale are imposed. The observable physical values at the TeV scale could then change significantly from their values at a high-energy scale. An example of such a scenario is provided by models with gravity-mediated Supersymmetry breaking. 


\subsection{The Minimal Supergravity Model (mSUGRA)}

The model of minimal SUperGRAvity (mSUGRA) is a GUT model. It is a supersymmetric model which does include gravity and the resulting theory is called Supergravity. In supersymmetric models which do not include gravitation, Supersymmetry is a global symmetry. If Supersymmetry is local it necessarily involves the introduction of gravity [18].

In mSUGRA the mechanism for spontaneous Supersymmetry breaking is analogous to the Higgs mechanism in gauge theories. At this scale there are only a few, independent universal parameters of Supersymmetry breaking.

The relation of the mSUGRA parameters to the masses and couplings of the MSSM is provided via renormalization group equation. Their initial conditions at the energy scale of $M_{G U T} \sim 10^{16} \mathrm{GeV}$ depend on the mechanism by which Supersymmetry breaking is communicated to the low-energy theory.

\subsubsection{Supersymmetry and Electroweak Breaking}

The most common scenario for producing low-energy Supersymmetry breaking is called the hidden sector scenario [19]. In this scenario, two sectors are assumed to exist:

the "hidden" sector in which the spontaneous Supersymmetry breaking occurs by adopting a Higgs mechanism and

the "visible" sector to which the Supersymmetry breaking is transmitted.

The visible sector contains all the MSSM particles, whereas the hidden sector contains particles that interact extremely weakly with the particles of the MSSM. The Supersymmetry breaking in the hidden sector of the theory is used to break the degeneracy of the quark and squark masses, etc., in the visible sector of the theory. The messenger of the Supersymmetry breaking is gravity.

The dynamic evolution from the scale of $M_{G U T}$ to the $M_{W}$ scale can deform the Higgs potential causing spontaneous breaking of the electroweak symmetry. The RG equations for the Higgs squared-mass $m_{H_{u}}^{2}$ decrease the Higgs mass when evolving the RG equation downwards to the electroweak scale and the large top quark Yukawa coupling can cause the $m_{H_{u}}^{2}$ to become negative. Therefore, the large top Yukawa coupling induces negative radiative corrections to the Higgs squared-mass and breaks the electroweak symmetry.

It is one of the remarkable aspects of mSUGRA, that it leads to the radiative breaking of the electroweak symmetry as a consequence of RG equation effects. 


\subsubsection{Parameters of mSUGRA}

The Supersymmetry breaking defined at a high energy scale is communicated to the MSSM via gravitational interactions. The soft-breaking terms of the MSSM Lagrangian can all be written in terms of only five independent Supersymmetry breaking parameters ${ }^{6}$ at the GUT scale [7]:

- a common scalar mass $\left(m_{0}\right)$, that gives the boundary condition for the masses of the supersymmetric spin-0 particles (sfermions) and Higgs squared-mass parameters,

- a common gaugino mass $\left(m_{1 / 2}\right)$, that gives the boundary condition for the masses of the supersymmetric spin-1/2 particles (gauginos),

- a common trilinear interaction $\left(A_{0}\right)$,

- a common B-term $\left(B_{0}\right)$,

- a Higgs mass parameter $\mu$.

Renormalization group evolution is used to compute the low-energy values of the mSUGRA parameters. This fixes the various MSSM masses and couplings at the electroweak scale $M_{W}$. Because of the ignorance of the supersymmetry breaking mechanism, the parameters $m_{0}, m_{1 / 2}, A_{0}$ and $B_{0}$ are usually taken to be independent for phenomenological studies.

The two Higgs vacuum expectation values can be expressed as a function of the mSUGRA parameters: the Higgs mass parameter $\mu$ and the bilinear coupling $B_{0}$ are removed in favor of $m_{Z}$ and $\tan \beta$, whereas the sign of $\mu$ is not fixed in this process. The rich pattern of sparticle masses and couplings of the MSSM at the electroweak scale $M_{W}$ is then determined in terms of a commonly used parameter set [11, 7]:

$$
m_{0}, \quad m_{1 / 2}, \quad A_{0}, \quad \tan \beta, \quad \text { and } \operatorname{sign}(\mu) .
$$

The choice of these five parameters at the energy scale $M_{G U T}$ will define the masses and coupling of the MSSM at the electroweak scale.

The radiative corrections which are applied to the various masses and couplings evolved from the GUT scale to the electroweak scale yield the mechanism for the breaking of electroweak gauge symmetry, leaving color and electromagnetic symmetries unbroken. A model with spontaneous local Supersymmetry breaking in the hidden sector which is communicated to the visible sector via gravitational interactions, is therefore equal to a model without gravitation and soft breaking terms introduced "by hand".

\footnotetext{
${ }^{6}$ The scale at which these parameters are specified is frequently chosen to be the GUT scale.
} 


\subsubsection{Constraints of the mSUGRA Model}

The mSUGRA model has five parameters (compare Eq. 2.7) that define the masses and couplings in the MSSM. These parameters are to be fitted by experimental constraints.

The constraints of the MSSM itself follow from the comparison of the predictions with experimental data (also limits from non-observation of sparticles) and from the idea of Supersymmetry GUT models. All of these constraints can be fulfilled simultaneously in the MSSM. Some are listed below:

Gauge coupling constant unification. This is one of the most restrictive constraints. It fixes the supersymmetric soft terms at an order of $1 \mathrm{TeV}$.

Mass of the $Z$ boson from electroweak symmetry breaking. Radiative corrections trigger spontaneous electroweak symmetry breaking. To get the correct value of $m_{Z}$ requires proper adjustment of parameters. This condition determines the value of $\mu$ for given values of $m_{0}$ and $m_{1 / 2}$.

The branching fraction and photon energy spectrum for $b \rightarrow s \gamma$ has been measured to yield $B R(b \rightarrow s \gamma)=(3.21 \pm 0.27) \cdot 10^{-4}$ [20]. The Standard Model prediction is very close to the experimental value, leaving little space for Supersymmetry. This requirements imposes restrictions on the parameter space, especially for the case of large $\tan \beta$.

Experimental lower limits on sparticle masses. Sparticles have not been observed so far. From the searches at LEP, lower limits on the charged slepton and chargino masses of about half of the center of mass energy have been set [21]. The lower limit on the neutralino masses is less stringent. The constraints on the mSUGRA model were calculated by using the results of numerous analyses. Among them: searches for pair production of supersymmetric particles: $\tilde{e}, \tilde{\tau}, \widetilde{\chi}_{1}^{ \pm}$, searches for the neutral scalar light Higgs $h^{0}$ in the process $e^{+} e^{-} \rightarrow h^{0} Z^{0}$, the consequences of the results obtained from electroweak precision measurements [21, 22].

The dark matter constraint. The LSP (which is stable), would have been abundantly produced in the early universe. From cold dark matter searches, which assume that the LSP is the dominant form of dark matter in the galactic halo, limits have been derived based on the lack of detection in laboratory experiments [23]. There are also bounds on the LSP from astrophysics and cosmology, requiring that the LSP contribution to the overall cosmological density is less than some maximal value to avoid over-closure of the universe [24].

The mSUGRA model is a simple and phenomenologically viable framework that offers distinctly testable consequences from a small parameter set. It serves as a paradigm for the Supersymmetry search in this dissertation. 


\subsection{Phenomenology of mSUGRA}

The mSUGRA parameters are starting values for the coupled set of renormalization group equations at the GUT scale. The resulting gauge, Yukawa and soft Supersymmetry breaking parameters, at the electroweak scale, can be related to the soft breaking terms of the MSSM.

A schematic mass spectrum of the sparticles of the mSUGRA model is shown in figure 2.2. Variations of the mSUGRA parameters at the GUT scale can modify the diagram substantially. For example, choosing the scalar mass parameter $m_{0}$ very small can minimize the mass spectra of the squarks, sleptons and Higgs bosons and as a consequence that of the neutralinos, charginos and gluinos.

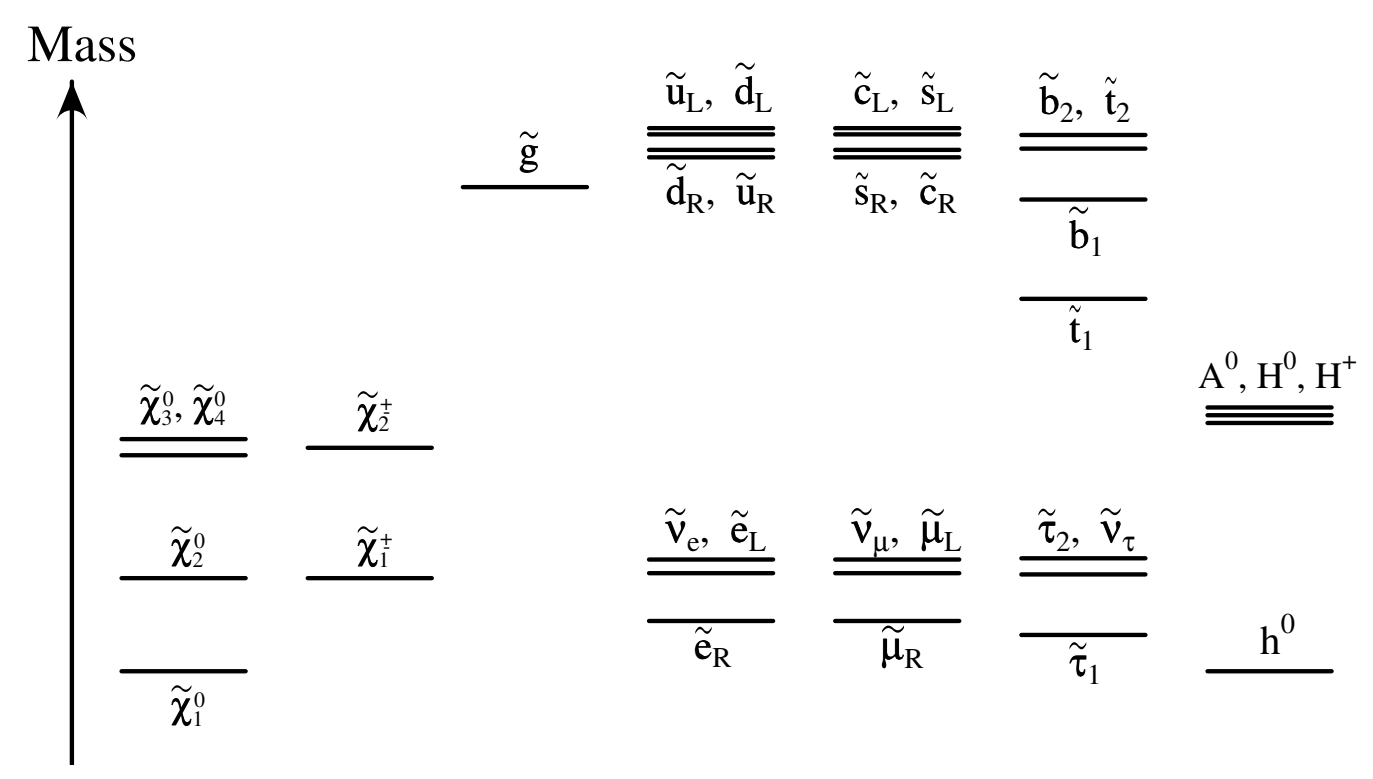

Figure 2.2: Schematic mass spectra for the sparticles. This spectrum is presented for illustrative purpose only [7].

In the mSUGRA model, $\widetilde{\chi}_{1}^{0}$ is the lightest supersymmetric particle appearing at the end of every supersymmetric particle decay. The $\widetilde{\chi}_{1}^{0}$ escapes experimental detection, resulting in apparent non-conservation of energy/momentum in Supersymmetry events.

A more detailed description of the sparticle masses, their production and decay is given in the following, focusing on the supersymmetric signatures important for this dissertation.

\subsubsection{Squarks and Sleptons}

The supersymmetric partners of the quarks and leptons are spin- 0 bosons: squarks $\widetilde{q}$, charged sleptons $\tilde{\ell}$ and sneutrinos $\tilde{v}$. For a given fermion $f$, there are two sfermions $\tilde{f}_{L}$ and $\tilde{f}_{R}$. They are scalar partners corresponding to the left- and right-handed fermion. (There is no $\tilde{v}_{R}$ in the mSUGRA model or in the MSSM.) 
In general, $\tilde{f}_{L}$ and $\tilde{f}_{R}$ are not the mass eigenstates, since there is $\tilde{f}_{L}-\tilde{f}_{R}$ mixing proportional in strength to the corresponding element of the scalar squared mass matrix [7]. In principle, any scalars with the same electric charge, R-parity and color quantum number can mix. Table 2.3 lists all the sfermions indicating the mass and gauge eigenstates.

\begin{tabular}{|c|c|c|c|c|c|}
\hline Names & Spin & $R$ & "Mass Eigenstates & "Gauge Eigenstates & "Generations \\
\hline \multirow{3}{*}{ squarks } & \multirow{3}{*}{$1 / 2$} & \multirow{3}{*}{-1} & $\begin{array}{llll}\tilde{u}_{L} & \tilde{u}_{R} & \tilde{d}_{L} & \tilde{d}_{R}\end{array}$ & “ & $1 \mathrm{st}$ \\
\hline & & & $\begin{array}{llll}\tilde{s}_{L} & \tilde{s}_{R} & \tilde{c}_{L} & \tilde{c}_{R}\end{array}$ & “ & $2 \mathrm{nd}$ \\
\hline & & & $\begin{array}{llll}\tilde{t}_{1} & \tilde{t}_{2} & \tilde{b}_{1} & \tilde{b}_{2}\end{array}$ & $\begin{array}{llll}\tilde{t}_{L} & \tilde{t}_{R} & \tilde{b}_{L} & \tilde{b}_{R}\end{array}$ & $3 \mathrm{rd}$ \\
\hline \multirow{3}{*}{ sleptons } & \multirow{3}{*}{$1 / 2$} & \multirow{3}{*}{-1} & $\tilde{e}_{L} \tilde{e}_{R} \tilde{\mathrm{v}}_{e}$ & “ & $1 \mathrm{st}$ \\
\hline & & & $\tilde{\mu}_{L} \tilde{\mu}_{R} \tilde{v}_{\mu}$ & “ & $2 n d$ \\
\hline & & & $\begin{array}{lll}\tilde{\tau}_{1} & \tilde{\tau}_{2} & \tilde{v}_{\tau}\end{array}$ & $\begin{array}{lll}\tilde{\tau}_{L} & \tilde{\tau}_{R} & \tilde{v}_{\tau}\end{array}$ & $3 \mathrm{rd}$ \\
\hline
\end{tabular}

Table 2.3: The sfermions in the mSUGRA model. The index L,R of the sleptons and squarks refer to the helicity of their Standard Model partners. The mass eigenstates are also the gauge eigenstates for the 1 st and 2 nd generation sfermions. $R$ is the R-parity value.

The first and second generation squarks and sleptons have negligible Yukawa couplings. They are grouped in seven nearly degenerate, unmixed pairs $\left(\tilde{e}_{R}, \tilde{\mu}_{R}\right),\left(\tilde{\mathrm{v}}_{e}, \tilde{\mathrm{v}}_{\mu}\right)$, $\left(\tilde{e}_{L}, \tilde{\mu}_{L}\right),\left(\tilde{u}_{R}, \tilde{c}_{R}\right),\left(\tilde{d}_{R}, \tilde{s}_{R}\right),\left(\tilde{u}_{L}, \tilde{c}_{L}\right),\left(\tilde{d}_{L}, \tilde{s}_{L}\right)$.

The third generation squarks and sleptons can have very different masses compared to their first and second generation counterparts, because of large Yukawa couplings. Additionally they can have substantial mixing in the pairs $\left(\tilde{t}_{R}, \tilde{t}_{L}\right),\left(\tilde{b}_{R}, \tilde{b}_{L}\right)$ and $\left(\tilde{\tau}_{R}, \tilde{\tau}_{L}\right)$. The resulting mass eigenstates are $m_{\tilde{t}_{1}}<m_{\tilde{t}_{2}}, m_{\tilde{b}_{1}}<m_{\tilde{b}_{2}}$, and $m_{\tilde{\tau}_{1}}<m_{\tilde{\tau}_{2}}$. The tau Yukawa coupling for large $\tan \beta$ is important in the evolution of the stau slepton masses and mixings. If $\tan \beta$ is not too large (this means less than 10), the effect of mixing on the sbottom and staus is not large. In this case the mass eigenstates are nearly the same as the gauge eigenstates $\tilde{b}_{R}, \tilde{b}_{L}, \tilde{\tau}_{R}, \tilde{\tau}_{L}$ and the latter two plus the $\tilde{v}_{\tau}$ will be nearly degenerate with their first and second-family counterparts with the same $\mathrm{SU}(3)_{\text {color }} \times \mathrm{SU}(2)_{\text {left }} \times \mathrm{U}(1)_{\mathrm{Y}}$ quantum numbers.

If $m_{0}$ and $m_{1 / 2}$ are of the same order of magnitude, physical sfermion masses are dominantly given by $m_{0}$. The RG evolutions determine their masses from the starting value $m_{0}$ down to the electroweak scale (see fig. 2.3). The colored particles are generally driven heavier at low-energy (Q) because of the large strong gauge coupling. The squark and slepton masses split at low energies and the lightest squark and slepton are third generation particles. In general, the squarks are heavier than the sleptons and the lightest neutralino and chargino [7]. 


\subsubsection{Gauginos, Higgsinos and the Higgs Bosons}

The supersymmetric partners of the gauge and Higgs bosons are spin-1/2 fermions: the gauginos bino $\widetilde{B}$, gluinos $\tilde{g}$, winos $\widetilde{W}$ and the higgsinos $\widetilde{H}_{u}, \widetilde{H}_{d}, \widetilde{H}_{u}^{+}, \widetilde{H}_{d}^{-}$. The gauginos and higgsinos can mix. As a result, the physical mass eigenstates are linear combinations of these states, called neutralinos and charginos. They are obtained by diagonalizing the corresponding mass matrices [11]. The neutralino mass matrix is given as

$$
\mathcal{M}_{\tilde{\chi}^{0}}=\left(\begin{array}{cccc}
M_{1} & 0 & -m_{Z} \cos \beta \sin \theta_{W} & m_{Z} \sin \beta \sin \theta_{W} \\
0 & M_{2} & m_{Z} \cos \beta \cos \theta_{W} & -m_{Z} \sin \beta \cos \theta_{W} \\
-m_{Z} \cos \beta \sin \theta_{W} & m_{Z} \cos \beta \cos \theta_{W} & 0 & -\mu \\
m_{Z} \sin \beta \sin \theta_{W} & -m_{Z} \sin \beta \cos \theta_{W} & -\mu & 0
\end{array}\right)
$$

where $\theta_{W}$ is the weak mixing angle, $M_{1}=m_{\widetilde{B}}$ and $M_{2}=m_{\widetilde{W}^{0}}$ are the gaugino masses associated with $\mathrm{U}(1)$ and $\mathrm{SU}(2), m_{Z}$ is the $Z$ boson mass and $\mu$ provides for the higgsino mass terms. The chargino mass matrix is given as

$$
\mathcal{M}_{\widetilde{\chi}^{ \pm}}=\left(\begin{array}{cc}
M_{2} & \sqrt{2} m_{W} \sin \beta \\
\sqrt{2} m_{W} \cos \beta & +\mu
\end{array}\right)
$$

where $m_{W}$ is the mass of the $W$ boson and $M_{2}=m_{\widetilde{W}^{ \pm}}$is the gaugino mass associated with $\mathrm{SU}(2)$. The mass and gauge eigenstates of the higgsinos, neutralinos and charginos are

\begin{tabular}{|c|c|c|c|c|}
\hline Names & Spin & $R$ & Mass Eigenstates & Gauge Eigenstates \\
\hline Higgs bosons & 0 & +1 & $h^{0} \quad H^{0} \quad A^{0} \quad H^{ \pm}$ & $H_{u} \quad H_{d} \quad H_{u}^{+} \quad H_{d}^{-}$ \\
\hline neutralinos & $1 / 2$ & -1 & $\begin{array}{lll}\tilde{\chi}_{2}^{0} & \widetilde{\chi}_{3}^{0} & \tilde{\chi}_{4}^{0}\end{array}$ & $\begin{array}{cccc}\widetilde{B} & \widetilde{W}^{0} & \widetilde{H}_{u} & \widetilde{H}_{d}\end{array}$ \\
\hline charginos & $1 / 2$ & -1 & $\tilde{\chi}_{1}^{ \pm} \quad \tilde{\chi}_{2}^{ \pm}$ & $\tilde{W}^{ \pm} \quad \tilde{H}_{u}^{+} \quad \tilde{H}_{d}^{-}$ \\
\hline
\end{tabular}
listed in table 2.4 with their associated quantum numbers ${ }^{7}$.

Table 2.4: The gauginos and higgsinos in the mSUGRA model with their mass and gauge eigenstates. $R$ is the R-parity value.

The starting value of the RG equations for the gaugino masses is $m_{1 / 2}$. This assumption of gaugino unification at the GUT scale, results in a simple relation between the gluino, chargino and neutralinos in the mSUGRA model:

$$
2 m_{\widetilde{\chi}_{1}^{0}} \cong m_{\widetilde{\chi}_{1}^{ \pm}} \cong m_{\widetilde{\chi}_{2}^{0}} \simeq \frac{1}{3} m_{\tilde{g}}
$$

In the Higgs sector the masses of $A^{0}, H^{0}$ and $H^{ \pm}$can be arbitrarily large, whereas the lightest Higgs boson $h^{0}$ is bound from above in the MSSM by quantum corrections with $m_{h^{0}} \lesssim 130 \mathrm{GeV}[7]$.

\footnotetext{
${ }^{7}$ In the mass eigenstates, the charginos and neutralinos are labeled such that $m_{\widetilde{\chi}_{1}^{ \pm}}<m_{\widetilde{\chi}_{2}^{ \pm}}$and $m_{\widetilde{\chi}_{1}^{0}}<$ $m_{\widetilde{\chi}_{2}^{0}}<m_{\widetilde{\chi}_{3}^{0}}<m_{\widetilde{\chi}_{4}^{0}}$ for $M_{1}<M_{2}$.
} 
The Higgs squared-mass parameters $m_{H_{u}}^{2}$ and $m_{H_{d}}^{2}$ assume the boundary condition of $m_{0}$ at the GUT scale with the starting value of $m_{0}+\mu^{2}$. One of the Higgs masses is driven negative giving the electroweak symmetry breaking (fig. 2.3). An interesting property of the electroweak limit is that $m_{Z} \ll\left|\mu+M_{1}\right|,\left|\mu+M_{2}\right|$ is valid over most of the parameter space of the theory. This implies that the light neutralinos $\widetilde{\chi}_{1}^{0} \widetilde{\chi}_{2}^{0}$ and the lightest charginos $\widetilde{\chi}_{1}^{ \pm}$are mostly gauginos, and the heavy states mostly higgsinos.

Figure 2.3 shows a typical evolution of the soft-Supersymmetry breaking parameters from the GUT scale to the electroweak scale. The gauginos $\widetilde{B}, \widetilde{W}, \tilde{g}$ have masses of

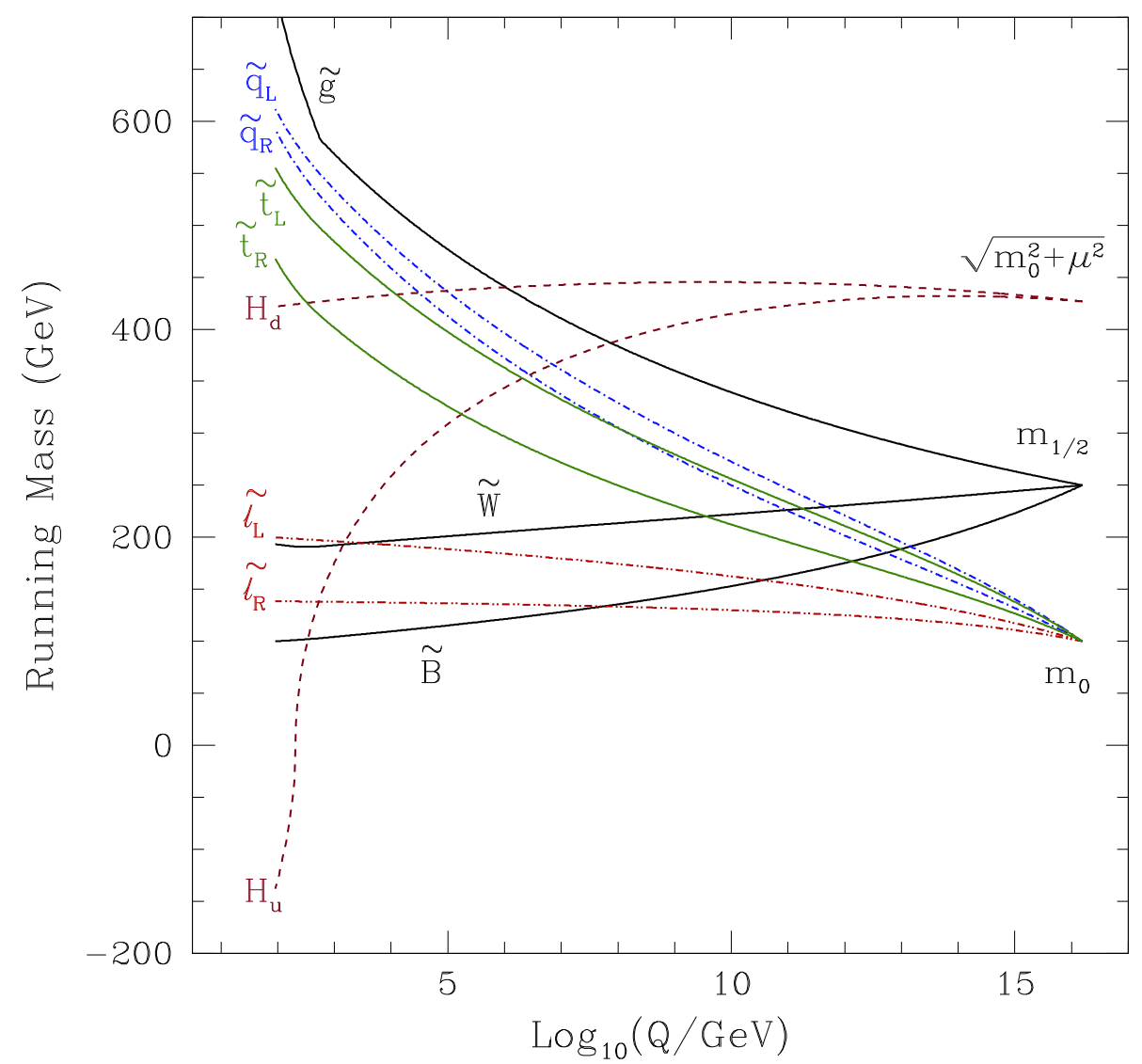

Figure 2.3: An example of the RG evolution for a particular point in the mSUGRA parameter space: $m_{1 / 2}=250 \mathrm{GeV}, m_{0}=100 \mathrm{GeV}, \tan \beta=3, A_{0}=0, \mu<0$. [25]

$m_{1 / 2}$ at the GUT scale. They follow the running of the gauge couplings and split at low energies. The gluino mass runs faster than the others and is usually the largest due to strong interaction. The sfermions start at a mass value of $m_{0}$ at the GUT scale and split into the squarks and the sleptons. The right-handed sleptons especially right-handed stau $\tilde{\tau}_{R}$ ) are typically the lightest scalars. The lightest neutralinos and charginos are rather light. 


\subsubsection{Creation and Decay of Supersymmetric Particles}

At hadron colliders, in particular at the Tevatron collider where protons and anti-protons collide, the dominant production mechanisms for supersymmetric particles are by $q \bar{q}$ annihilation and scattering, gluon fusion and quark gluon scattering [11, 7, 26]. The production of gaugino pairs is possible via annihilation reactions: $q+\bar{q} \rightarrow \widetilde{\chi}_{i}^{ \pm}+\widetilde{\chi}_{j}^{\mp}$ and $q+\bar{q}^{\prime} \rightarrow \widetilde{\chi}_{i}^{ \pm}+\widetilde{\chi}_{j}^{0}$. The Feynman graphs are shown in figure 2.4.
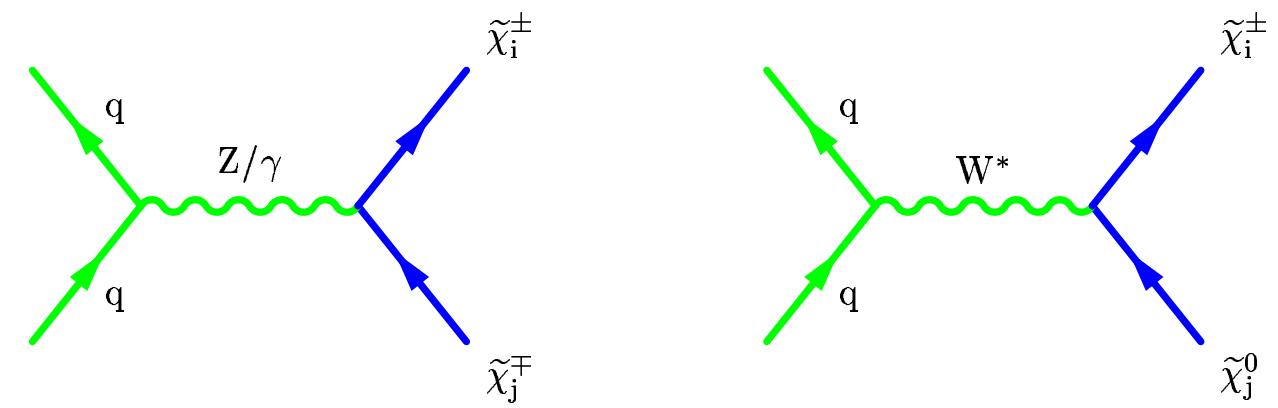

Figure 2.4: Creation of gaugino pairs via $q \bar{q}$ annihilation. The sparticles are colored in blue, the ordinary particles in green.

The decay properties of the sparticles depend on their masses. The lighter sparticles will have the simplest decays, while the heavier sparticles will decay to the lighter ones. The decay chain ends with the $\widetilde{\chi}_{1}^{0}$ in the final state.

\subsubsection{Decays of Squarks and Sleptons}

The heavy squarks and sleptons often decay to charginos and neutralinos. Squarks can decay via: $\widetilde{q} \rightarrow q+\widetilde{\chi}_{i}^{0}, \quad \widetilde{q} \rightarrow q^{\prime}+\widetilde{\chi}_{i}^{ \pm}$, where $q \widetilde{q}$ is a (s)quark-anti(s)quark pair. Figure 2.5 (left) shows a squark decay. The decays of the sleptons are: $\tilde{\ell}^{ \pm} \rightarrow \ell^{ \pm}+\widetilde{\chi}_{i}^{0}$,
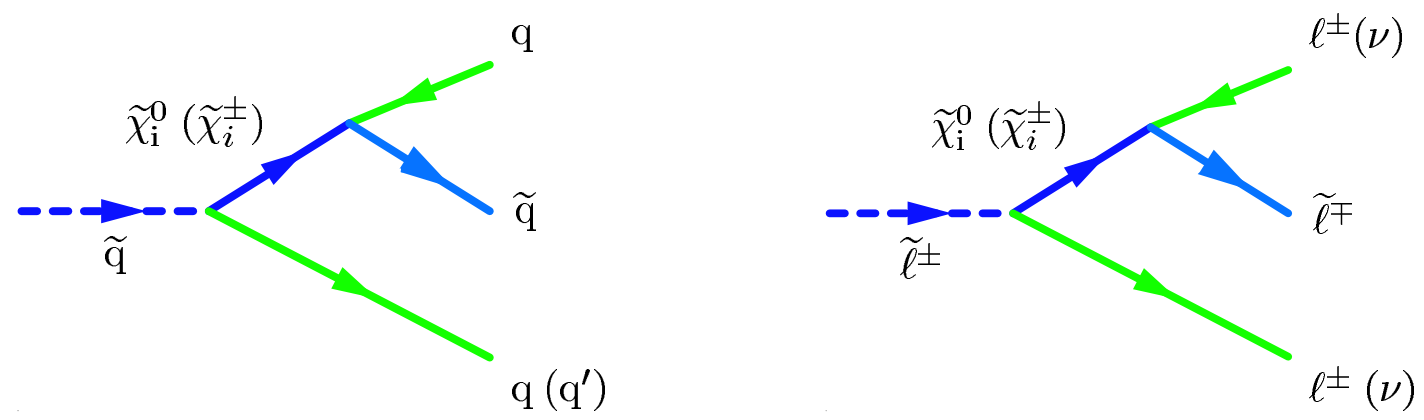

Figure 2.5: Possible decay of a squark (left) and a slepton (right) via gaugino interaction.

$\tilde{\ell}^{ \pm} \rightarrow v+\tilde{\chi}_{i}^{ \pm}$, where $\ell \tilde{\ell}$ is a (s)lepton-anti(s)lepton pair with $\ell=e, \mu, \tau$, and $v=v_{e}, v_{\mu}, v_{\tau}$. Figure 2.5 (right) shows a slepton decay. The Feynman diagrams of the sneutrino decays $\tilde{v} \rightarrow v+\widetilde{\chi}_{i}^{0}$ and $\tilde{v} \rightarrow \ell^{ \pm}+\widetilde{\chi}_{i}^{ \pm}$are not shown here. The direct decays into the $\widetilde{\chi}_{1}^{0}$ are always kinematically allowed if the $\widetilde{\chi}_{1}^{0}$ is the lightest supersymmetric particle. 


\subsubsection{Decays of Neutralinos and Charginos}

Each neutralino and chargino contains at least a small admixture of the electroweak gauginos (bino and wino) as seen in section 2.5.2. So the $\widetilde{\chi}^{ \pm}$and the $\widetilde{\chi}^{0}$ inherit couplings of weak interaction strength to scalar-fermion pairs as seen above in the squark and slepton decays. If sleptons or squarks are light, a neutralino (chargino) can decay into a lepton+slepton or quark+squark. As sleptons are lighter than squarks, for most of the parameter space, the lepton+slepton final states are expected to be dominant. A neutralino (chargino) may also decay into any lighter neutralino (chargino) plus an electroweak gauge boson. Therefore the most probable two-body decays are ${ }^{8}$ :

$$
\begin{array}{llll}
\text { for } \widetilde{\chi}^{0}: & \widetilde{\chi}_{i}^{0} \rightarrow \ell^{ \pm} \tilde{\ell}^{\mp}, \widetilde{\chi}_{j}^{0} Z & \text { and } & \widetilde{\chi}_{i}^{0} \rightarrow \widetilde{\chi}_{j}^{ \pm} W^{\mp} \\
\text { for } \widetilde{\chi}^{ \pm}: & \widetilde{\chi}_{i}^{ \pm} \rightarrow v \tilde{\ell}^{ \pm}, \widetilde{\chi}_{j}^{ \pm} Z & \text { and } & \tilde{\chi}_{i}^{ \pm} \rightarrow \widetilde{\chi}_{j}^{0} W^{ \pm}
\end{array}
$$

where $\tilde{\ell}$ are sleptons and $v, \ell$ denote neutrinos and charged leptons. For the heavier chargino and neutralinos $\left(\widetilde{\chi}_{2}^{ \pm}, \widetilde{\chi}_{3}^{0}, \widetilde{\chi}_{4}^{0}\right.$ ) one or more of the above decays are kinematically allowed.

If these two-body decays are kinematically forbidden, then the three-body decays are possible through the same (but now off-shell) gauge bosons and sleptons that appeared in the two-body decays. The decays

$$
\begin{aligned}
& \tilde{\chi}_{1}^{ \pm} \rightarrow \tilde{\chi}_{1}^{0} W^{*}\left(\tilde{\ell}^{*}\right) \rightarrow \tilde{\chi}_{1}^{0} \ell^{ \pm} v \\
& \tilde{\chi}_{2}^{0} \rightarrow \widetilde{\chi}_{1}^{0} Z^{*}\left(\tilde{\ell}^{*}\right) \quad \rightarrow \quad \ell^{+} \ell^{-} \widetilde{\chi}_{1}^{0}
\end{aligned}
$$

are particularly important for phenomenology, because this final state has a clean leptonic signal. Figure 2.6 shows the three-body decays (or two-body decay via real gauge bosons or sleptons, if kinematically allowed) in the case of the chargino $\widetilde{\chi}_{1}^{ \pm}$. The decay for the neutralino $\widetilde{\chi}_{2}^{0}$ is similar.
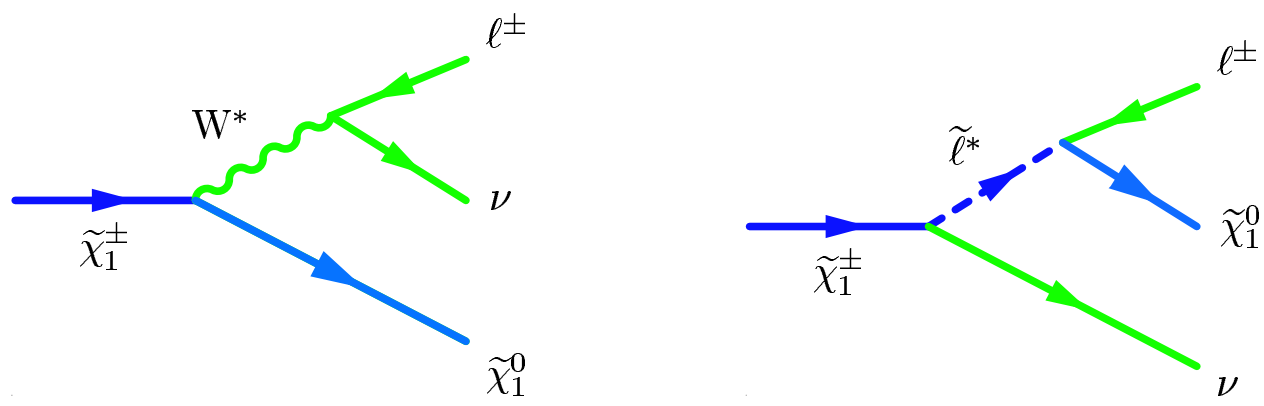

Figure 2.6: Chargino three-body decay through virtual boson (left) and virtual slepton decay (right).

Because of the clean signal, the decays of $\widetilde{\chi}_{1}^{ \pm} \widetilde{\chi}_{2}^{0}$ into leptonic final states are especially important at hadron colliders. The Supersymmetry search performed in this dissertation focuses on such decays.

\footnotetext{
${ }^{8}$ Only the decays which are important for the later analysis are presented here. Decays via a lighter gaugino plus a Higgs scalar, or decays involving sneutrinos are possible. See [7, 11] for further reading.
} 


\subsubsection{The Trilepton Signature at the Tevatron}

In mSUGRA, the sleptons, the lighter chargino $\left(\widetilde{\chi}_{1}^{ \pm}\right)$and the lighter neutralinos $\left(\widetilde{\chi}_{1}^{0}, \widetilde{\chi}_{2}^{0}\right)$ are typically less massive than the gluinos and the squarks. In a typical scenario, where the $\widetilde{\chi}_{1}^{ \pm}$ and $\widetilde{\chi}_{2}^{0}$ are mostly gaugino-like and the $\widetilde{\chi}_{1}^{0}$ is mostly bino-like, the largest production cross sections belong to the $\widetilde{\chi}_{1}^{ \pm} \widetilde{\chi}_{1}^{\mp}$ and $\widetilde{\chi}_{1}^{ \pm} \widetilde{\chi}_{2}^{0}$ channels because they have significant couplings to the $\gamma, Z$ and $W^{ \pm}$bosons, respectively. Figure 2.7 shows the production cross section for chargino neutralino pair production at the center-of-mass energy of the Tevatron as a function of the gaugino masses.

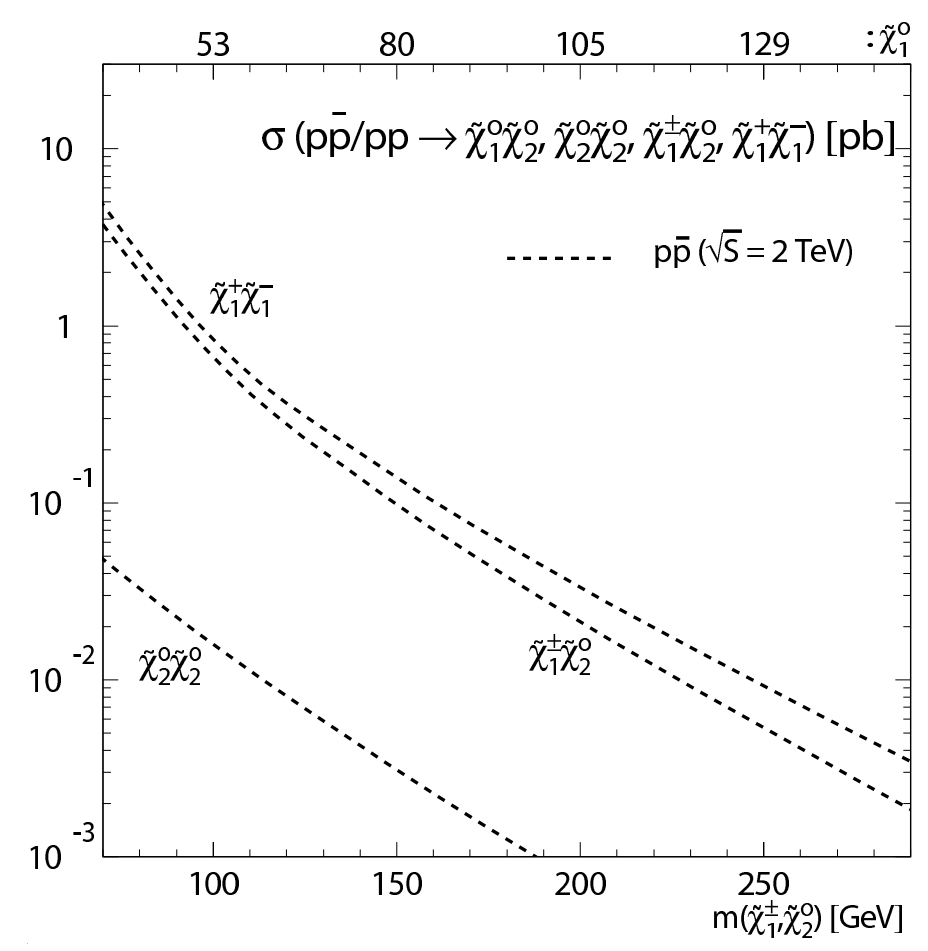

Figure 2.7: NLO cross section for chargino neutralino pair production as a function of the gaugino masses [27].

This dissertation focuses on the associated production of the lightest chargino along with the second lightest neutralino $q \bar{q} \rightarrow \widetilde{\chi}_{1}^{ \pm} \widetilde{\chi}_{2}^{0}$. This is in the kinematic reach of the Tevatron and can be produced with observable cross sections. In the mSUGRA model the squark masses are comparable to or greater than the gluino masses. In this model the squark pair production is most likely beyond the Tevatron reach.

The produced sparticles decay into final states with two LSPs $\left(\widetilde{\chi}_{1}^{0}\right)$, which escape the detector. The resulting missing energy which is carried away by the two $\widetilde{\chi}_{1}^{0}$, can be observed at hadron colliders only by the missing momentum transverse to the colliding beams. It is called missing transverse energy $\left(\not_{T}\right)$.

The $q \bar{q} \rightarrow \widetilde{\chi}_{1}^{ \pm} \widetilde{\chi}_{2}^{0}$ production occurs via quark-antiquark annihilation through a $W^{*}$ boson in the s-channel and through $\widetilde{q}^{*}$ exchange in the t-channel. The produced sparticles 
decay via virtual gauge bosons or sleptons into the final state with three leptons $\left(\ell^{ \pm}\right)$, two $\widetilde{\chi}_{1}^{0}$ and one neutrino $(v)$. This final state is often referred to as the trilepton SUSY signal:

$$
q \bar{q} \rightarrow \widetilde{\chi}_{1}^{ \pm} \widetilde{\chi}_{2}^{0} \rightarrow \widetilde{\chi}_{1}^{0} v \ell^{ \pm} \widetilde{\chi}_{1}^{0} \ell^{ \pm} \ell^{\mp}
$$

As an example figures 2.8 and 2.9 show the relevant Feynman graphs, for the neutralino decay into muons (the decays into electrons and taus are equivalent) and the chargino decay into three possible flavors of the leptons and their associated neutrinos.
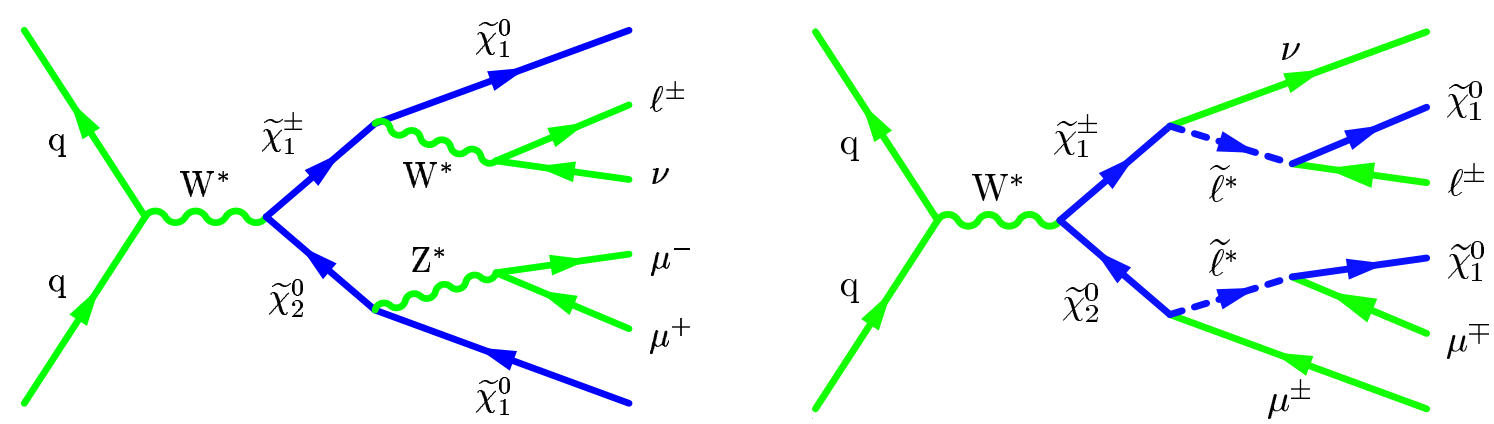

Figure 2.8: Feynman graphs of $q \bar{q} \rightarrow \widetilde{\chi}_{1}^{ \pm} \widetilde{\chi}_{2}^{0} \rightarrow \ell^{ \pm} v \widetilde{\chi}_{1}^{0} \mu^{ \pm} \mu^{\mp} \widetilde{\chi}_{1}^{0}$ via the s-channel.
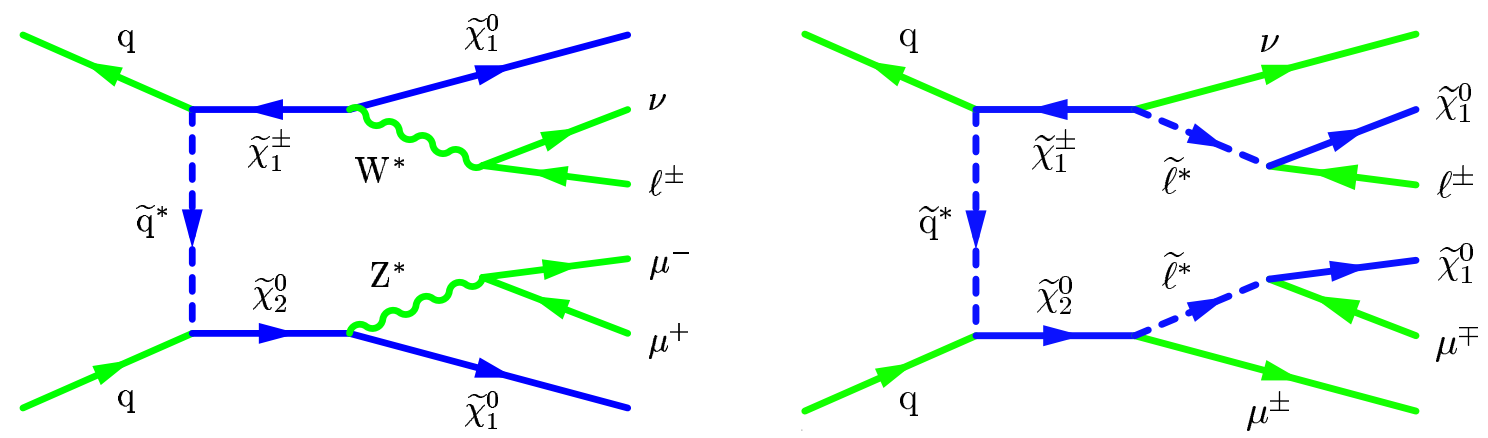

Figure 2.9: Feynman graphs of $q \bar{q} \rightarrow \widetilde{\chi}_{1}^{ \pm} \widetilde{\chi}_{2}^{0} \rightarrow \ell^{ \pm} v \widetilde{\chi}_{1}^{0} \mu^{ \pm} \mu^{\mp} \widetilde{\chi}_{1}^{0}$ via the t-channel.

If the squarks are light, a destructive interference between the $W^{*}$ boson and the $\widetilde{q}^{*}$ exchange amplitudes can suppress the total cross section by as much as $40 \%$ compared to the s-channel contribution alone. For squarks much heavier than the gauge bosons, the effect of negative interference is reduced and the s-channel $W^{*}$-resonance amplitude dominates.

\subsubsection{Cross Section}

When considering universal boundary conditions at the GUT scale, the masses of the gauginos are defined by a common gaugino mass $m_{1 / 2}$ and the masses of the sleptons are 
defined by a common scalar mass $m_{0}$. Additionally the mass of the stau sleptons are very sensitive of the value of $\tan \beta$.

The $\widetilde{\chi}_{1}^{ \pm} \widetilde{\chi}_{2}^{0}$ decay via virtual sleptons or gauge bosons into the stable $\widetilde{\chi}_{1}^{0}$ and leptons. The sleptons produced in the $\widetilde{\chi}_{2}^{0}$ or $\widetilde{\chi}_{1}^{ \pm}$decay can be of three flavors: $\tilde{e}, \tilde{\mu}, \tilde{\tau}$. They decay further into the $\widetilde{\chi}_{1}^{0}$ and a lepton of the same flavor: $e, \mu, \tau,\left(v_{e}, v_{\mu}, v_{\tau}\right)$ respectively.

The production cross section of the trilepton signal $q \bar{q} \rightarrow \widetilde{\chi}_{1}^{ \pm} \widetilde{\chi}_{2}^{0} \rightarrow 3 \ell+2 \widetilde{\chi}_{1}^{0}+\mathrm{v}$ at the center-of-mass energy of the Tevatron collider is shown in figure 2.10 as a function of $\tan \beta[26]$.

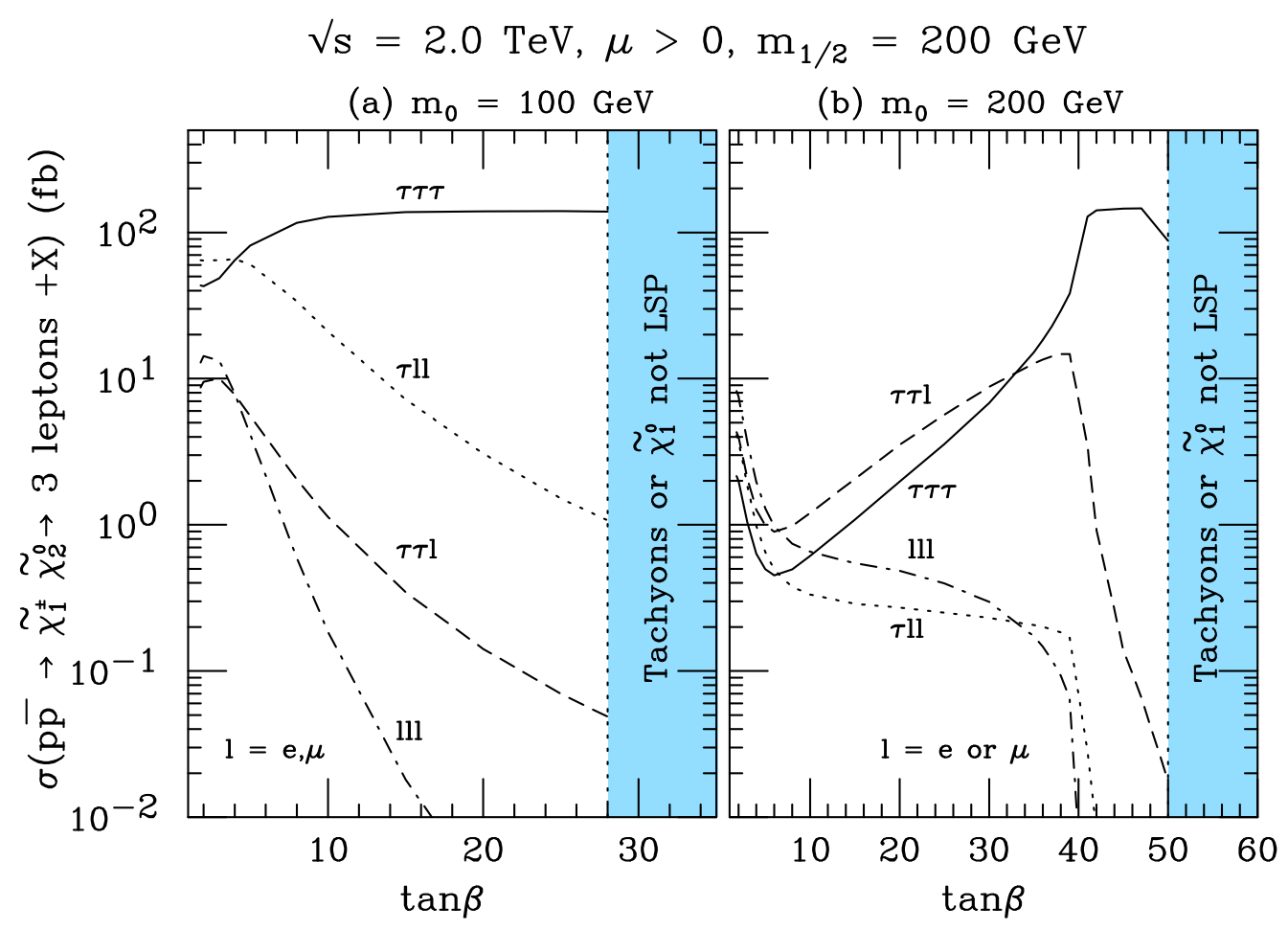

Figure 2.10: The production cross section for $q \bar{q} \rightarrow \widetilde{\chi}_{1}^{ \pm} \widetilde{\chi}_{2}^{0} \rightarrow 3 \ell+2 \widetilde{\chi}_{1}^{0}+v$ as a function of $\tan \beta$. The label on the $y$-axis " 3 leptons $+X$ " corresponds to $3 \ell+2 \widetilde{\chi}_{1}^{0}+v[26]$.

As $\tan \beta$ increases, the light stau ( $\left.\tilde{\tau}_{1}\right)$ becomes lighter than charginos and neutralinos while other sleptons and squarks are heavy. Then, $\tilde{\chi}_{1}^{ \pm}$and $\widetilde{\chi}_{2}^{0}$ decay dominantly into final states with tau leptons via real $\tilde{\tau}_{1}$. If excellent $\tau$ identification becomes available, than the decay channels with at least two taus might be a promising way to search for $\widetilde{\chi}_{1}^{ \pm} \widetilde{\chi}_{2}^{0}$.

At low $\tan \beta$ the search for $\widetilde{\chi}_{1}^{ \pm} \widetilde{\chi}_{2}^{0}$ in final states with electrons and muons can also be performed successfully. The values of the cross section can change significantly with the variation of the mSUGRA parameters.

In this dissertation, the final state $q \bar{q} \rightarrow \widetilde{\chi}_{1}^{ \pm} \widetilde{\chi}_{2}^{0} \rightarrow \ell^{ \pm} \vee \widetilde{\chi}_{1}^{0} \mu^{ \pm} \mu^{\mp} \widetilde{\chi}_{1}^{0}$ with two muons, an additional charged lepton and $\mathbb{E}_{T}$ is investigated. The investigated decay channel is most sensitive to the parameter space of $\tan \beta \sim 3, m_{0}<100 \mathrm{GeV}$ and $m_{1 / 2}<200 \mathrm{GeV}$. In this case the chargino and neutralino mostly decay into final states with two electrons 
or muons and one additional charged lepton. For $m_{0} \geq 200 \mathrm{GeV}$ and for higher values of $\tan \beta$ the cross section and therefore the sensitivity decreases evidently for the investigated decay channel.

\subsection{Summary}

This chapter gave a brief introduction into the theory of the mSUGRA model, emphasizing on its properties which are important for the presented analysis. It can be briefly summarized:

- The Standard Model of elementary particle physics is a very successful model considering its predictions and descriptions of the particles and their interactions. Nevertheless it is not sufficient to be the ultimate description of nature. It appears as an effective model at low energies accessible with accelerators.

- Supersymmetry introduces a new symmetry which associates known bosons with new fermions and known fermions with new bosons. The new particles are supersymmetric. It is a possible model, that answers at least some of the questions left unsolved by the Standard Model. Supersymmetry provides a mechanism for electroweak symmetry breaking.

- The Minimal SuperSymmetric Model is the supersymmetric extension of the Standard Model introducing a minimal number of supersymmetric parameters.

The mSUGRA model introduces gravitation into a supersymmetric theory and relates its five parameters to the soft parameters of the MSSM. The values of these five free parameters are constrained by experimental results.

- The trilepton channel is also referred to as "golden channel" to search for Supersymmetry at the Tevatron. In this particular analysis the final state

$$
q \bar{q} \rightarrow \widetilde{\chi}_{1}^{ \pm} \widetilde{\chi}_{2}^{0} \rightarrow \ell^{ \pm} \vee \widetilde{\chi}_{1}^{0} \mu^{ \pm} \mu^{\mp} \widetilde{\chi}_{1}^{0}
$$

which will be referred to as

$$
\widetilde{\chi}_{1}^{ \pm} \widetilde{\chi}_{2}^{0} \rightarrow \mu \mu \ell+\mathbb{E}_{T}
$$

with two muons, an additional charged lepton and missing transverse energy $\mathbb{E}_{T}$ is investigated. 


\section{Chapter 3}

\section{Experimental Overview}

The Tevatron collider is the final stage in a chain of accelerators that create and gradually accelerate protons and anti-protons. At two designated interaction points, around which the general purpose detectors CDF and $\mathrm{D} \emptyset$ are built, the protons and anti-protons are brought to collision. The Tevatron collider provided first collisions in 1985 with a center-of-mass energy $(\sqrt{\mathrm{s}})$ of $1.8 \mathrm{TeV}$. Between 1996 and 2001 the accelerator complex experienced a major upgrade. Currently it operates a at a center-of-mass energy of $1.96 \mathrm{TeV}$ referred to as Tevatron Run II. Figure 3.1 shows an aerial view of Fermilab. The accelerators are located below the surface. However the general layout is clearly visible due to the roads and cooling water ponds following the ring on ground level.

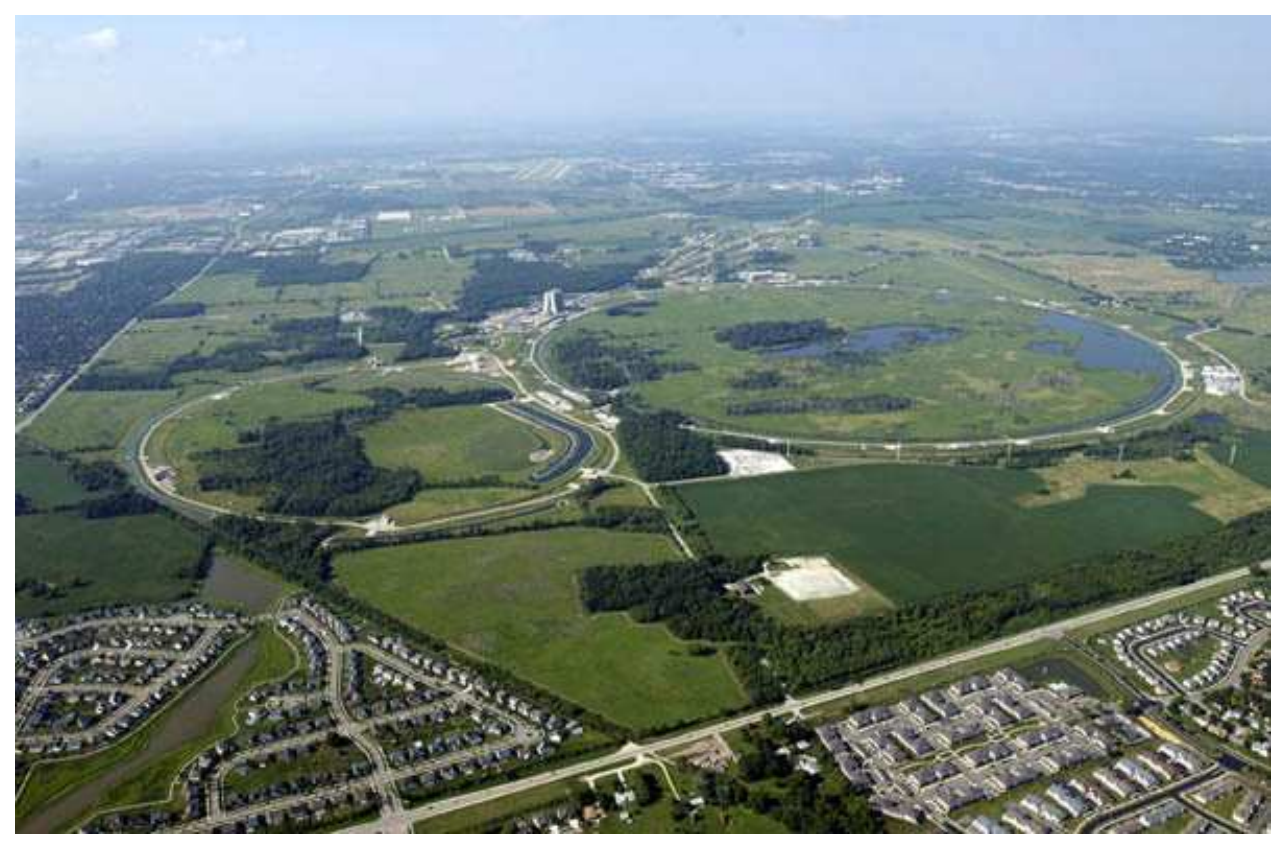

Figure 3.1: Aerial view of the Tevatron accelerator chain. 


\subsection{Fermilab Accelerator Chain}

For Run II the Tevatron collider has been upgraded to achieve higher instantaneous luminosity and a higher beam energy of $0.98 \mathrm{TeV}$ per beam. An integrated luminosity of $4-8 \mathrm{fb}^{-1}$ per experiment is expected until 2009 depending on the accelerator performance. The Tevatron ring is fed proton and anti-proton beams from a complex chain of seven other accelerators (fig. 3.2).

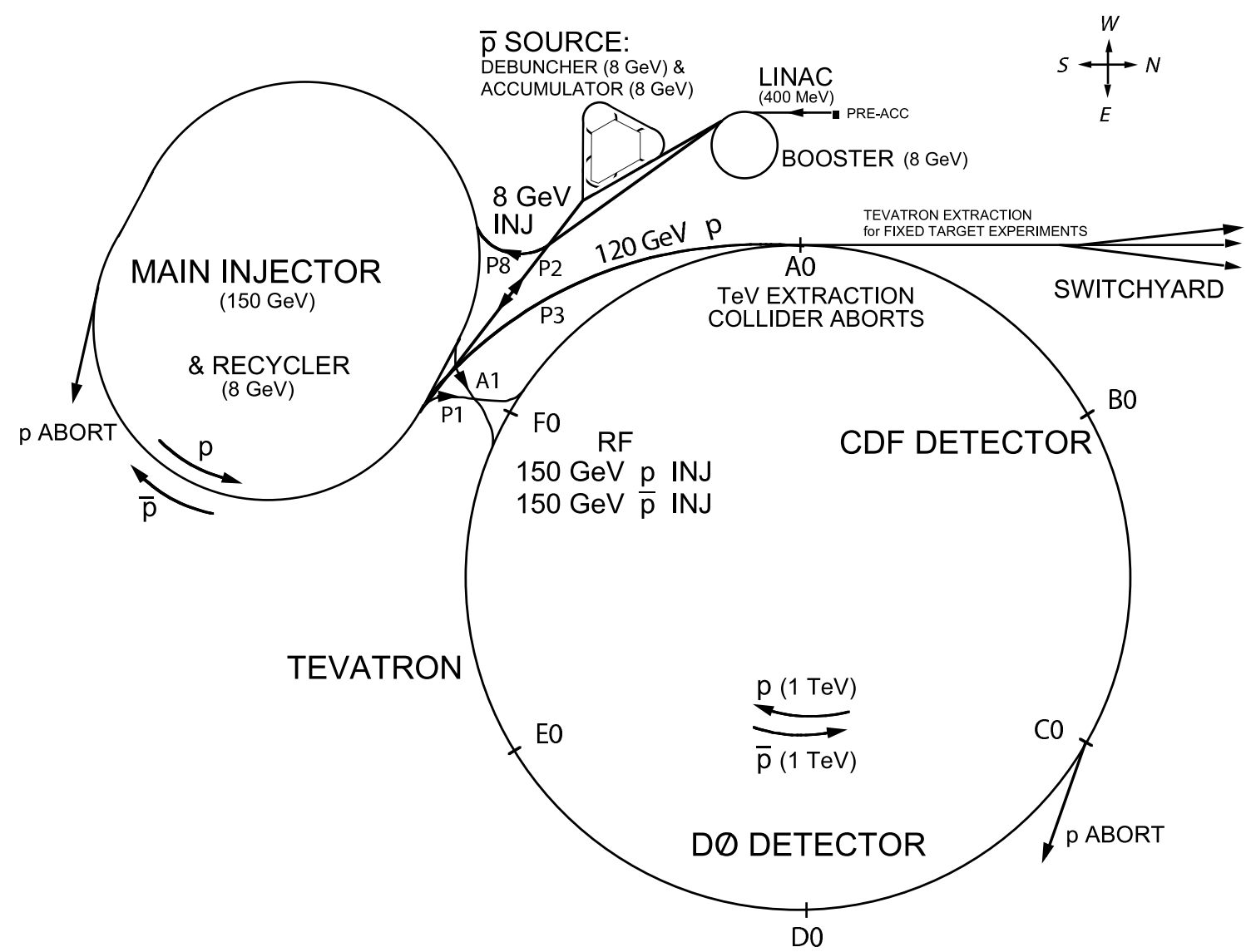

Figure 3.2: The Tevatron accelerator chain.

The accelerator chain for protons starts at the Cockcroft-Walton pre-accelerator, where negative hydrogen ions $\mathrm{H}^{-}$are accelerated to energies of $750 \mathrm{keV}$. The ions pass the second stage of acceleration in a linear accelerator (Linac) of approximately 150 meters length where oscillating electric fields accelerate the $\mathrm{H}^{-}$ions to $400 \mathrm{MeV}$. Before entering the third stage of acceleration, the booster, electrons are stripped off as the ions pass through a carbon fiber foil. The booster is the first synchrotron (of six in total) in the accelerator chain at Fermilab. It is $\mathbf{4 7 5}$ meters in circumference and accelerates protons to $8 \mathrm{GeV}$. The proton beam is then transfered to the Main Injector, a larger synchrotron ring. Here the protons are accelerated to $120 \mathrm{GeV}$ for fixed target operation or $150 \mathrm{GeV}$ for injection into the Tevatron. The Main Injector groups the protons and anti-protons into 
three bunches before delivering them to the Tevatron, where the bunches are accelerated to $0.98 \mathrm{TeV}$.

The anti-proton beam starts at the Antiproton Source. It consists of three major components: the Target Station, the Debuncher and the Accumulator (figure 3.3). The bunches of $120 \mathrm{GeV}$ protons from the Main Injector are directed at a $7 \mathrm{~cm}$ long nickel target, producing anti-protons. These are moved to the Debuncher storage ring, an $8 \mathrm{GeV}$ syn-

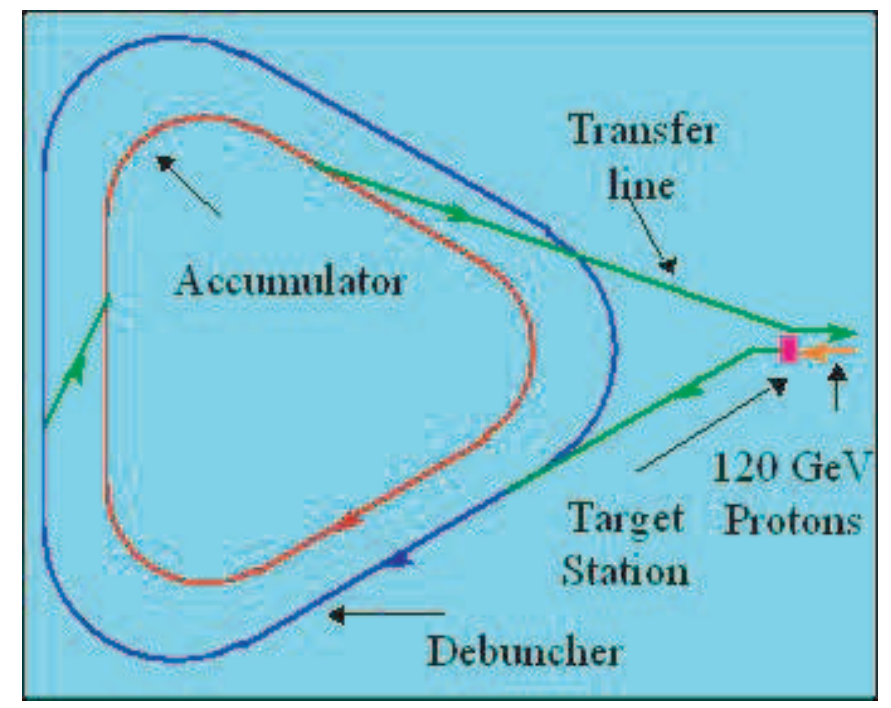

Figure 3.3: The Antiproton Source. Anti-protons are produced in the Target Station, focused in the Debuncher and stored in the Accumulator before beeing transfered to the Recycler.

chrotron, where they are formed into a coherent beam before being transferred to the Accumulator, another $8 \mathrm{GeV}$ synchrotron. Once sufficient numbers of anti-protons have been collected in the Accumulator, they are passed to the Recycler. The Recycler is a fixed-energy storage synchrotron and placed in the Main Injector tunnel directly above the Main Injector beamline. Once a sufficient number of anti-protons have been collected, they are passed to the Main Injector, where they are accelerated to $150 \mathrm{GeV}$. There are about 1000 superconducting magnets along the ring, each of which creates a magnetic field of 4.2 T. The currents through the superconducting magnet coil at $980 \mathrm{GeV}$ are exceedingly large, as much as 4000 Amperes. The coil wires are made of superconducting material (iobium-titanium alloy wire, of size $8 \mu \mathrm{m}$ ) kept at a temperature of 4.6 Kelvin to have zero resistance to the electric currents.

The Tevatron has six possible proton anti-proton collision points, labeled A0 - F0. The two detectors on the Tevatron ring are located at B0 (the CDF experiment) and D0 (the DØ experiment) [28]. 


\subsection{DØ Detector}

The $\mathrm{D} \varnothing$ detector is a multi-purpose particle detector designed to study proton anti-proton collisions. To do this, it is necessary to identify and measure the properties of particles produced in these collisions. Short lived particles decay before detection and are reconstructed from their decay products. Quarks or gluons are not observed directly, but form jets of hadrons.

In general, there are six distinct types of particles with lifetimes long enough to be detected: photons, electrons, muons, neutrinos as well as charged and neutral hadrons. Except for the neutrino which is measured as missing transverse momentum, the particles can be identified and measured by their different interactions with matter. For this purpose, the $\mathrm{D} \varnothing$ detector combines tracking, calorimetry and muon detection over a wide angular range. Figure 3.4 shows a two dimensional view of the detector.

The D $\varnothing$ detector has been designed to operate reliably and with small dead-times for high instantaneous luminosity in the Tevatron collider and bunch crossing times of $\Delta \mathrm{t} \sim 396 \mathrm{~ns}$. The design is based upon a beam structure with at least $2 \mu$ s gaps between bunches. A more detailed description of the $\mathrm{D} \varnothing$ detector is given in [29].

\section{DØ coordinates}

A right-handed coordinate system is used: the proton beam defines the $z$ direction, the $x$ axis points toward the center of the Tevatron ring and the $y$ axis points vertically. The $\mathrm{D} \varnothing$ detector is centered around the nominal interaction point $(0,0,0)$. The distribution of interaction points is Gaussian in z, centered at $z=0$ with an RMS of around $25 \mathrm{~cm}$.

The spherical coordinates are given by the azimuthal angle $\phi$, the polar angle $\theta$ and the radius $\mathrm{R}$. Because of the substructure of the proton, $\mathrm{D} \varnothing$ is generally not in the centerof-mass frame of each partonic collision, therefore $\theta$ is replaced by the pseudorapidity $\eta$, defined as

$$
\eta=-\ln (\tan (\theta / 2))
$$

The pseudorapidity is derived and is equal to rapidity for relativistic particles (i.e. most particles observed at $\mathrm{D} \varnothing)$. The $\eta$ coordinate is used in two ways: detector $\eta$ refers to the position of a particle relative to $z=0$; physics $\eta$ refers to the position of a particle relative to the actual $z$ position of the interaction point from which it came. For an undeflected particle originating at $z=0$ physics and detector $\eta$ are identical.

While the $\mathrm{z}$ momentum component of the colliding partons is not known, their transverse momentum $p_{T}=\sqrt{p_{x}^{2}+p_{y}^{2}}$ is generally small. The sum of transverse momenta of all particles in an event is then expected to sum up to approximately zero. Thus the transverse momentum is a useful parameter. Any undetected particles, such as neutrinos, become apparent as an imbalance in the total transverse momentum in an event. 


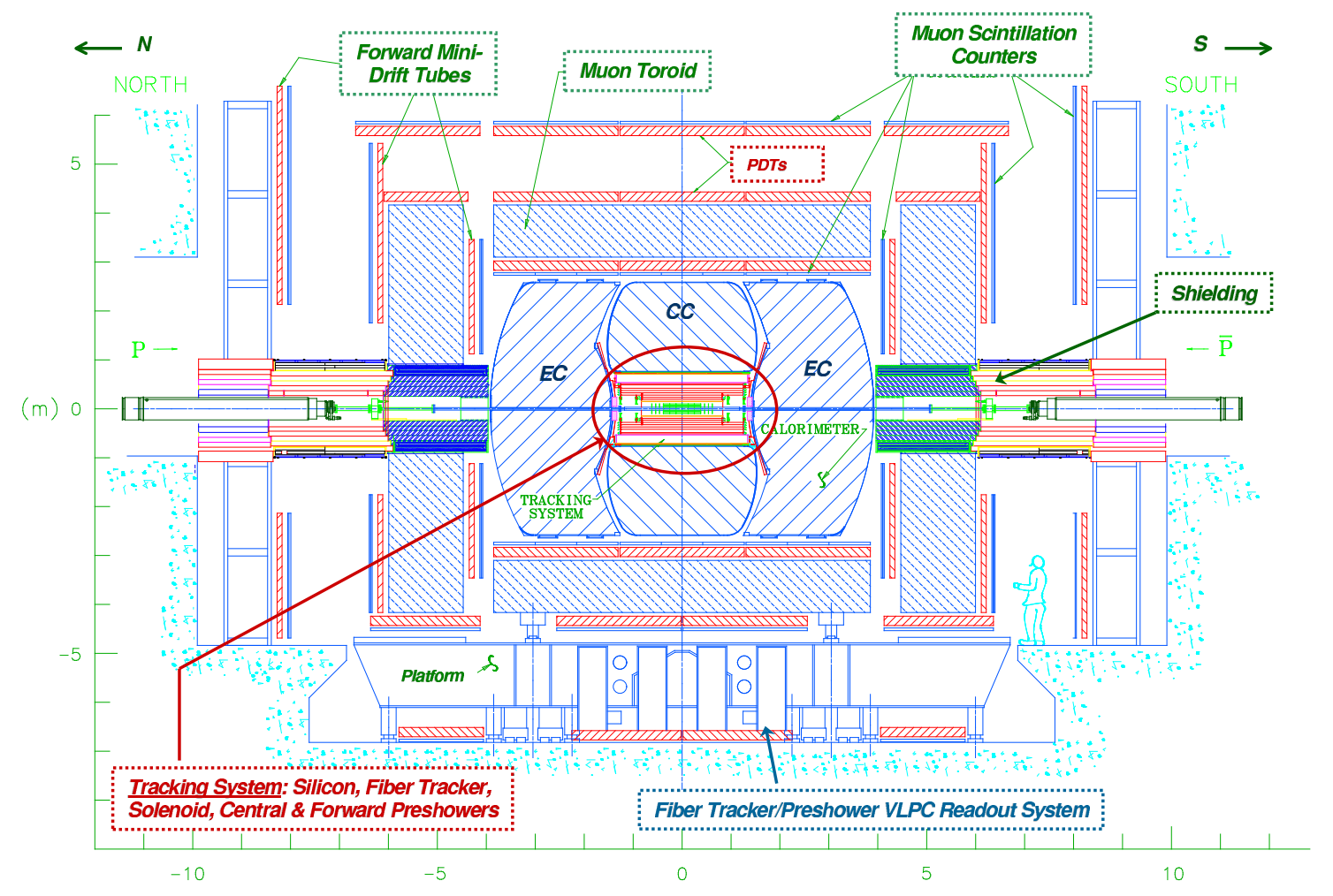

Figure 3.4: The two dimensional view of the $D \emptyset$ detector. 


\subsubsection{Tracking Detectors}

The paths of charged particles are reconstructed from the signals left in the D $\varnothing$ tracking detectors: the inner Silicon Micro-strip Tracker (SMT) and the outer scintillating Central Fiber Tracker (CFT). The two detectors components are located inside a solenoid with a radius of $60 \mathrm{~cm}$ and a length of $2.8 \mathrm{~m}$ which provides a magnetic field of $2 \mathrm{~T}$. A side view of the inner tracker with its solenoid is shown in figure 3.5 .

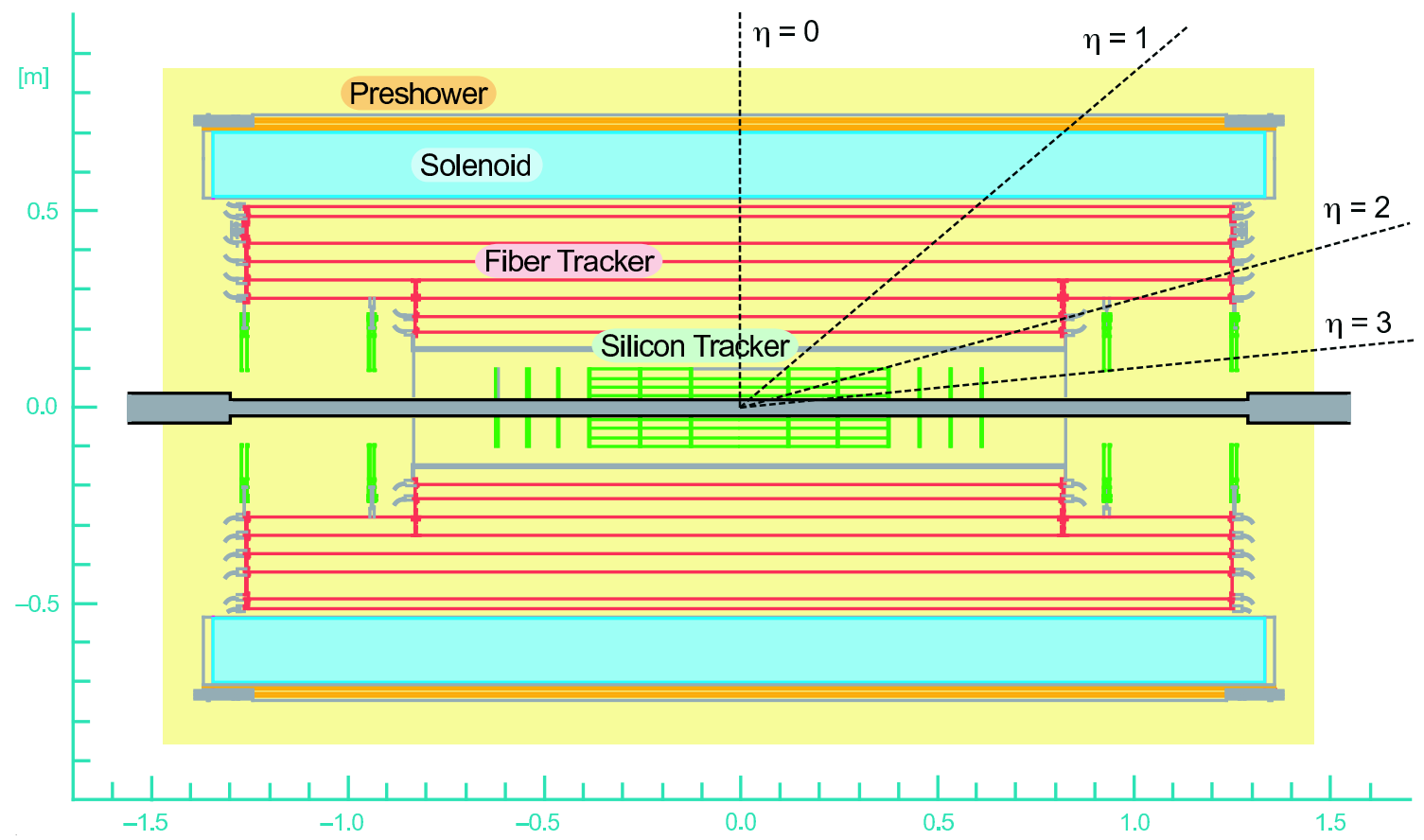

Figure 3.5: Side view of the inner tracker with its solenoid and the surrounding preshower detector.

The basic idea of the central tracking system is to yield accurate position measurements along the paths of charged particles. In the presence of the magnetic field of the solenoid, these paths will be curved. By measuring the curvature, the transverse momentum (the component of the particle momentum perpendicular to the magnetic field) can be extracted. The accuracy of this measurement is affected by the resolution of the individual position measurement and by the amount of multiple scattering. The desire to maximize the former and minimize the latter places requirements on the design and construction of the tracking system.

The momentum resolution expected for the $\mathrm{D} \varnothing$ tracking system can be parameterized for tracks at $\eta=0$ by: $\Delta p_{T} / p_{T}=0.02+0.002 p_{T}$ [29]. 


\section{The Silicon Micro-strip Tracker (SMT)}

The SMT is a high precision device surrounding the beam pipe in the center of DØ. The sensitive devices in the SMT are wafers of silicon, which is ionized by the passage of charged particles. The ionized charge is collected and used to measure the position of the passing particle.

Six cylindrical barrel detectors (three on either side of the interaction point) are arranged parallel to the beam direction in four layers of silicon wafers. They cover the region of $|z|<40 \mathrm{~cm}$ to provide the best tracking for particles at small $\eta$. The layers are evenly spaced between radii of $2.5 \mathrm{~cm}$ and $10 \mathrm{~cm}$.

Sixteen layers of wafers are placed perpendicular to the beam. Six of these disks are located between the barrels, with one at each end of the barrel detector. Two triplets are located at a small distance from either end of the barrel detector. The remaining disks cover a detection region of $9.6 \mathrm{~cm}<\mathrm{r}<23.6 \mathrm{~cm}$ around the beam pipe starting about one meter from the interaction point. The perpendicular layers improve tracking for particles at large $|\eta|$. The SMT allows tracking in a pseudo-rapidity range of $|\eta|<3$ and has about 800000 readout channels. The micro-strip detectors provide a hit resolution of approximately $10 \mu \mathrm{m}$.

\section{The Central Fiber Tracker (CFT)}

The CFT consists of eight cylindrical layers of carbon fiber support structure each holding two doublets of scintillating fibers between radii of $19.5 \mathrm{~cm}$ and $51.5 \mathrm{~cm}$ up to a length of $2.5 \mathrm{~m}$. Each doublet consists of two layers separated by $870 \mu \mathrm{m}$. The outer fiber in each doublet is offset by half a fiber width, improving the angular coverage. The fibers themselves are $835 \mu \mathrm{m}$ in diameter, giving a position resolution per doublet of $100 \mu \mathrm{m}$.

The passage of charged particles through the fiber causes scintillation. The scintillation light travels along the fiber in both directions. At one end, an aluminum mirror reflects the light back down the fiber. At the other end, the fiber is joined to a wavelength shifting waveguide which transmits the light to a solid state device called a Visible Light Photon Counter (VLPCs), located below the D $\varnothing$ detector, which converts the light into an electronic signal. The VLPCs have an optimum operating efficiency at $\approx 9 \mathrm{~K}$ and are held in a liquid helium cryostat.

Each of the eight layers holds one doublet running parallel to the beam (axial) providing a measurement of $\phi$ and $r$, and a stereo doublet rotated by $\pm 3^{\circ}$ providing stereo and thus $\mathrm{z}$ information. In total there are 77000 readout channels in the CFT.

\section{Preshower Detectors}

The preshower detectors use a mixture of tracking and calorimetry. The basic principle is to introduce some material before the preshower detectors to enhance the electron and photon identification by providing additional calorimetric measurement. Then, with sufficient detector resolution, it is possible to separate electromagnetic objects from hadrons. 
At $\mathrm{D} \varnothing$, the preshower detectors are located outside of the solenoid magnet: a central preshower detector (CPS) mounted on the solenoid and a forward preshower detector (FPS) on the inner surface of the end calorimeter cryostat. The forward detectors cover an angular region of $(1.4<|\eta|<2.5)$. The detectors are made of several layers of scintillating material. To induce showering, thin layers of lead are placed before the scintillators. As for the CFT, the scintillation light is collected through waveguides and transmitted to VLPCs located below the $\mathrm{D} \varnothing$ detector.

\subsubsection{Calorimeter}

The $\mathrm{D} \emptyset$ calorimeter provides particle identification and energy measurements by absorbing most particles. The energy of electrons, photons and jets is therefore measured directly. The calorimeter is a hermetic liquid argon sampling calorimeter. The basic unit is

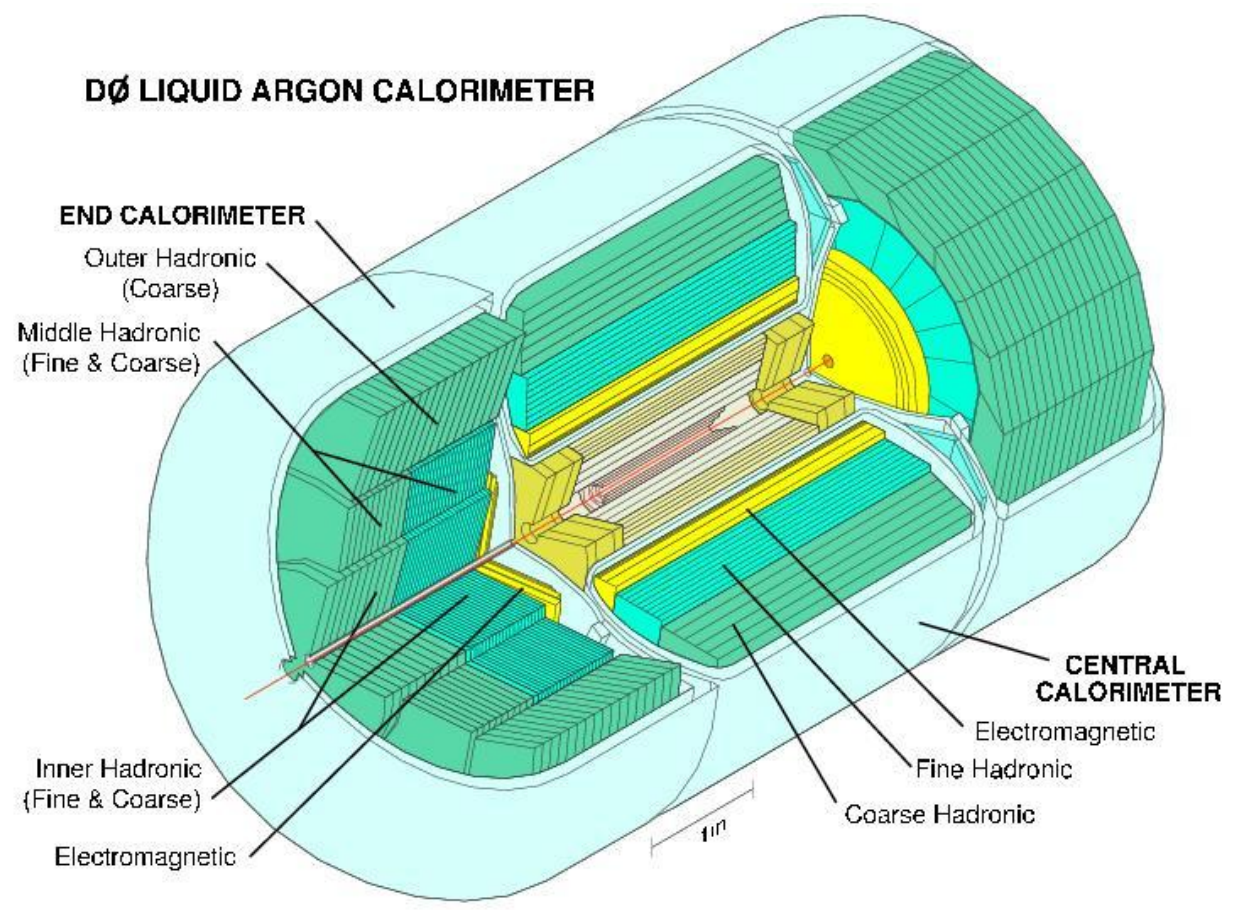

Figure 3.6: Three dimensional view of the liquid argon calorimeter.

a cell, consisting of layers of absorber material to induce the formation of particle showers and an active layer of liquid argon, which is ionized by the charged particles within the shower. The ionized charge represents a fraction of the energy of the shower and is collected on a copper plate within each cell. The total charge in all cells along the path of a shower can then be related to the total energy of the incoming particle.

The calorimeter is divided in a central and two forward regions with separate cryostat, covering a pseudo-rapidity range of $|\eta|<4$. Figure 3.6 shows a three dimensional view of 
the the whole calorimeter. Both the central and forward calorimeters are divided into three layers. In increasing distance from the interaction region, these are the electromagnetic, fine hadronic and coarse hadronic.

The central calorimeter is located outside the central preshower detector, extending from a radius of $72 \mathrm{~cm}$ to $220 \mathrm{~cm}$ with a pseudo-rapidity range of $|\eta|<1.2$. It consists of four electromagnetic layers, followed by three fine hadronic layers and one coarse hadronic layer. The forward (end) calorimeters lie beyond the forward preshower detectors in $\mathrm{z}$ and extend to $\mathrm{z}= \pm 400 \mathrm{~cm}$. They have an inner radius of $3 \mathrm{~cm}$ and an outer radius of $220 \mathrm{~cm}$ covering a pseudo-rapidity range of $1.4<|\eta|<4$. They consist of four electromagnetic layers, followed by three cylinders of hadronic layers (inner, middle and outer layer).

The position resolution is determined by the size of the cells in each layer, which cover a region of $0.1 \times 0.1$ in $\eta$ and $\phi$, except in the far forward region $(|\eta|<3.2)$, where the size is $0.2 \times 0.2$. The third layer of the electromagnetic calorimeter is more finely segmented at $0.05 \times 0.05$ to give improved resolution for electromagnetic showers. For trigger information the cells are formed into towers with a size of $0.2 \times 0.2$ and read out by separate electronics.

The energy resolution of the calorimeter for electrons and charged pions has been determined from test beam data to be:

$$
\begin{aligned}
\text { electrons : } \frac{\sigma(E)}{E} & =\left(\frac{15 \%}{\sqrt{E}}+0.3 \%\right) \\
\text { pions : } \frac{\sigma(E)}{E} & =\left(\frac{45 \%}{\sqrt{E}}+4 \%\right)
\end{aligned}
$$

where $\mathrm{E}$ is the energy in $\mathrm{GeV}$ of the detected particle.

The inter-cryostat detector (ICD) is located in the overlap region of the central and end calorimeter in a pseudo-rapidity region of $0.7<|\eta|<1.4$. It provides a correction for the energy loss in this region and improves the jet energy and missing transverse energy measurement. The detector consists of one single layer of 348 scintillating tiles each mounted on both end cryostat's. The detector signals are read out by photo-tubes which are connected by wavelength-shifting fibers.

\subsubsection{Muon System}

Muons pass through all of the inner detectors and calorimeter at $\mathrm{D} \varnothing$, losing only around $2.5 \mathrm{GeV}$ of their energy. Thus to identify muons a second tracking system, the muon detector, is located outside the calorimeter. The muon detector is separated into a central and a forward muon system. Additional iron shielding surrounds the beam pipe outside the calorimeter. This significantly reduces beam remnant activity in the forward muon system at large $\eta$. Figure 3.7 shows the outline of the D $\varnothing$ muon system [30].

The central muon system has three layers (called A, B, and C). The inner A layer, located just outside the calorimeter, and the outer B, C layers are separated by a toroid 


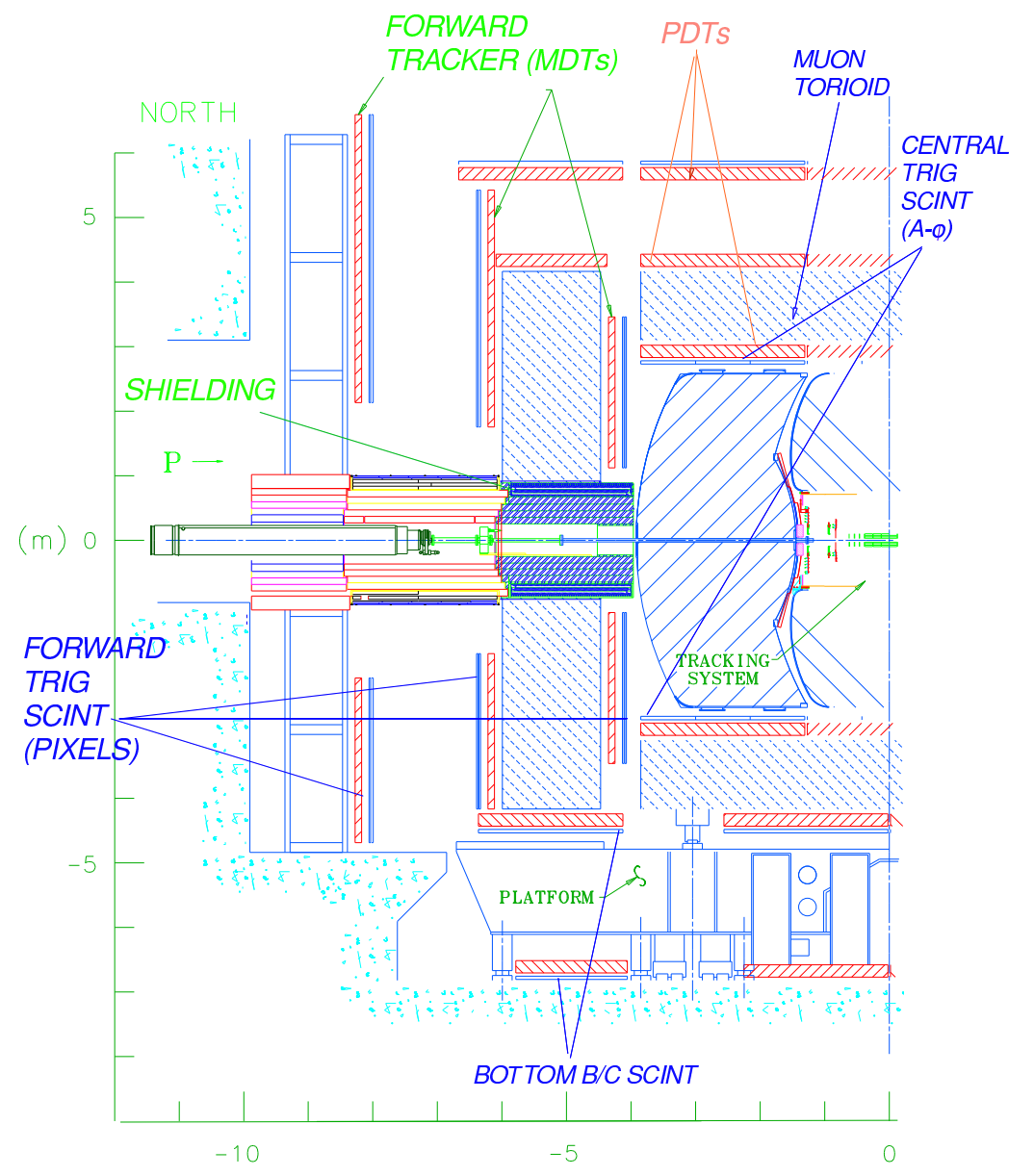

(m)

Figure 3.7: Side view of the Muon system of DØ. 
magnet of $1.8 \mathrm{~T}$ field, allowing a momentum measurement. The central muon system provides a coverage up to $|\eta|<1$. Its layers are composed of proportional drift tube chambers (PDTs) giving position measurements. The PDTs collect the charge which is ionized in the gas by the passage of a charged particle. The gas is held in a sealed volume, with the charge collected on high voltage wires running through the chamber. The PDTs are made of extruded aluminum of rectangular shape with a size of $5.7 \times 10 \mathrm{~cm}$. The anode wires are made of gold-plated tungsten. The PDTs have a drift time of $750 \mathrm{~ns}$ which is longer than the bunch crossing time of the Tevatron. Therefore, two different types of scintillation counters assure the trigger output: the A- $\phi$-counters, a layer of scintillators between the calorimeter and the A-layer PDTs which in addition reject out-of-time cosmic rays and scattered particles from the calorimeter, and the cosmic caps mounted outside the C-layer of the PDTs. There is a gap in the bottom part of the central muon system, due to the support structure of the $\mathrm{D} \emptyset$ detector. This region is only partly covered with scintillator counters.

The forward muon system also consists of three layers (A,B,C), except that these are composed of mini drift tubes (MDTs), and three layers of scintillation counters. The forward muon system extends the detector coverage up to $|\eta|<2$. As for the central muon system, the toroid separates the A layer from the layers B and C. The MDTs are composed of eight $10 \times 10 \mathrm{~mm}^{2}$ cells of extruded aluminum combs. All cells have an anode wire of radius $50 \mu \mathrm{m}$ placed in the center of the cell. Though the drift time of the MDTs is approximately $60 \mathrm{~ns}$, the scintillation counters are mostly used for triggering and for rejection of cosmic rays and other sources of background. They are mounted on the inside (layers A and C) and outside (layer B) of the MDT chambers. Time resolutions of around $2.5 \mathrm{~ns}$ are expected for the scintillation counters depending on their size.

The momentum resolution of the muon system is limited by multiple scattering at low momentum. It is parametrized as $\sigma_{\mathrm{p}} / \mathrm{p}=0.18+0.003 \mathrm{p}$ for high momentum and limited by the individual hit resolution. For the regions with the CFT coverage, the central tracking detectors provide a much better measurement of the momentum. However, in the forward region with SMT coverage only, the muon system measurement is comparable in resolution.

\subsubsection{Trigger and Data Acquisition System}

The rate of $p \bar{p}$ collisions at the Tevatron is significantly higher than the rate at which events can be read out and stored for offline analysis. Therefore, one of the major challenges involved in running an experiment such as $\mathrm{D} \emptyset$ is to select which events to store for further physics analysis. DØ uses a three level trigger system, with each level rejecting some events and passing others. The input rate of $2.5 \mathrm{MHz}$ given by the bunch crossing time of $396 \mathrm{~ns}$ is reduced to an event rate of $50 \mathrm{~Hz}$, which is written to tape.

The event selection is based on identifying physics objects (such as particles and tracks), with each level of the trigger system applying more detailed criteria. Figure 3.8 shows an outline of the Trigger System. 


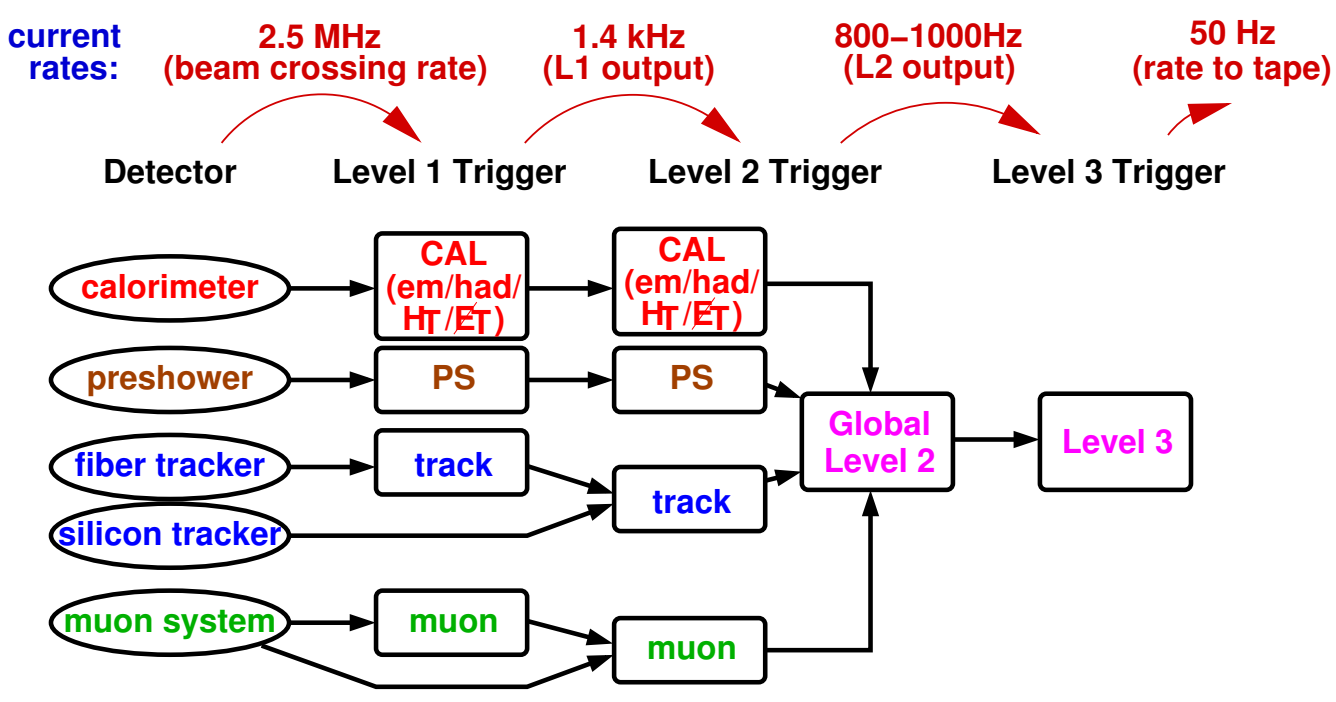

Figure 3.8: The 3 Level Trigger system of DØ.

For certain trigger conditions not all signals of a specified trigger are accepted, but only a predifined fraction. This method is called 'pre-scaling' and is used in the commissioning phase of a trigger or if the input rate is too high for the Data Acquisition System (DAQ). In the presented analysis, only events taken by triggers with no such pre-scaling are used for further analysis. The selection of events accepted by pre-scaled triggers does not increase the integrated luminosity of the data sample but introduces additional systematics.

\section{Level 1 Trigger}

The first trigger level (L1) is based on specialized fast readout, which gives approximate measurements to make a decision on each bunch crossing. The input rate of $2.5 \mathrm{MHz}$ is reduced to approximately $1.4 \mathrm{kHz}$. All detector systems other than the silicon tracker provide some information for the Level 1 trigger, e.g. energy measurement for L1 calorimeter and preshower, tracks consistent with e, $\mu$ and jets.

\section{Level 2 Trigger}

The Level 2 (L2) trigger also uses specialized fast readout, combining information from all detector components into an event trigger decision. The silicon and fiber tracking detectors provide triggers for particle tracks with different momentum requirements. The preshower and calorimeter triggers refine the energy measurement from L1 and the muon system uses information from both the scintillators and drift chambers, including position, momentum, charge and timing information. At an input rate of $1.4 \mathrm{kHz}$, a L2 decision happens within $100 \mu \mathrm{s}$. The L2 event rate is reduced to approximately $(0.8-1.0) \mathrm{kHz}$. 


\section{Level 3 Trigger}

The Level 3 (L3) trigger is software based, with a farm of processors carrying out partial event reconstruction. It reduces the event rate to approximately $50 \mathrm{~Hz}$. Events passing L3 are written to storage tapes for full offline event reconstruction. Unlike the previous trigger levels, L3 uses the full precision readout of the detector. The reconstruction software is similar to the offline reconstruction.

\subsubsection{Muon Trigger}

The presented analysis uses events containing at least two muon objects. The muon trigger [31] is used to select these events. An event is written to tape if the muon requirements at L1 and L2 are fulfilled for at least two muon objects. A fraction of events also passes the track requirement at $\mathrm{L} 3$.

\section{Level 1 Muon trigger}

The L1 muon triggers rely on information of the scintillation counters and the wire chambers. An event is passed on to the next trigger level if there is a coincidence of two layers in the same region and in the same octant of the muon system. Scintillators are calibrated such that a muon coming from a collision would reach them at a time $t=0$. For a trigger accept, a trigger gate of $|t|<20 \mathrm{~ns}$ for hits in the muon system is allowed.

\section{Level 2 Muon trigger}

Inputs to the L2 muon system are first received by second level input computers (SLICs), where most of the processing is performed. Tracks in the muon system are found by combining scintillator and wire chamber hits in each of the A, B and C layers. Finally muon objects are formed including position, momentum, and timing information.

The reconstructed muons are classified by their quality. In this analysis only events where at least one muon of 'medium' quality is found at L2, are passed on to the Level 3 muon trigger. To fulfill the 'medium' quality a muon reconstructed in the forward muon system must have at least two wire chamber hits in the A layer and at least one associated hit in the scintillator, for muons in the B and $\mathrm{C}$ layer a muon must have at least two hits, with hits in only one of the two layers. Muons reconstructed in the central muon system must have at least three wire chamber hits with a valid hit pattern for the $\mathrm{A}$ and $\mathrm{BC}$ layer.

\section{Level 3 Muon trigger}

With increasing instantaneous luminosity and better performance of the Tevatron, triggers having only L1 and L2 requirement for muon objects have been pre-scaled. An additional track requirement has been added in order to avoid the pre-scaling of the events. The tracking algorithm [32] first searches for CFT axial tracks and extrapolates them to CFT 
and SMT stereo clusters. Events pass the L3 Muon trigger if at least one global track with a transverse momentum of $5 \mathrm{GeV}$ is found.

\subsubsection{Luminosity Monitor}

In an inelastic $p \bar{p}$ collision, the remnants of the proton and anti-proton will typically be detected by the luminosity monitors, consisting of scintillating material placed around the beam pipe at $\mathrm{z}= \pm 140 \mathrm{~cm}$. They provide a coverage of $2.7<|\eta|<4.4$. When there is a coincidence of hits within $9 \mathrm{~ns}$ in the monitors on either side of the interaction region, the relative hit times also give a measurement of the z-position of the collision point.

The instantaneous luminosity is measured by the rate of inelastic collisions:

$$
\mathcal{L}=\frac{1}{\sigma_{p \bar{p}, e f f}} \frac{d N}{d t}(p \bar{p})
$$

with $\sigma_{p \bar{p}, e f f}=\varepsilon_{L M} \cdot A \cdot \sigma_{p \bar{p}}$. The luminosity detector efficiency $\varepsilon_{L M}$ is extracted from data by studying the calorimeter energy in the cells directly behind the luminosity detectors. $A$ corresponds to the luminosity detector acceptance determined using Monte Carlo and $\sigma_{p \bar{p}}$ is the inelastic $p \bar{p}$ cross section measured by other experiments to be $60.7 \pm 2.4 \mathrm{mb}$ [33]. The overall estimated error on the DØ luminosity is 6.5\% [34]. 


\section{Chapter 4}

\section{Monte Carlo and Data Samples}

The Standard Model background processes and the trilepton signal (SUSY signal process) are produced as Monte Carlo simulations. The data sample as well as the Standard Model background and the SUSY signal are described in the following including the description of the physics objects in an event (e.g. muons, tracks, jets, missing transverse energy).

\subsection{Event Simulation}

The generation of simulated events is carried out in three steps. As a first step, the $p \bar{p}$ collision is simulated for a specific process such as the $Z$ boson production with its decay into muons, or the production of the SUSY signal process, both used in this dissertation. An event generator, such as PYTHIA 6.202 [35] is used to implement the parton distribution functions [36], to carry out the matrix-element integration, hadronization, fragmentation and decay of short lived particles. Hadronization can be handled by the same event generator, but it is also possible to generate the events with one event generator and carry out the hadronization with another. In the analysis presented here the input parameters needed by PYTHIA for the generation of the SUSY signal process at the electroweak scale are obtained using ISAJET 7.58 [37], as included in the susy_tool interface [38].

In a second step the particles produced in the first step are passed through a GEANT detector simulation [39]. It calculates the effects on the particles which are due to the magnetic fields and the material in the detector. All events are then overlaid with a Poissondistributed average of 0.8 minimum-bias events.

The last step simulates the detector response of the $\mathrm{D} \emptyset$ detector components to the particles [40]. The output is used as input for the digitization of each detector, pileup (overlapping min-bias events) and raw data simulation [41]. The output is simulated data as produced by the real detector during a collision and must be reconstructed into physics objects with the same reconstruction software that is used for the data events. 


\subsection{Monte Carlo Samples}

\subsubsection{SUSY Signals}

The generated events for the SUSY signal process represent a mass region beyond the exclusion limits of direct searches for supersymmetric particles at LEP2. Including the results of the Higgs searches, neutralino masses below $47 \mathrm{GeV}$ are excluded in constrained MSSM models for negligible mixing in the stau sector and a lower limit of $42 \mathrm{GeV}$ was obtained for models with any mixing in the stau sector. In the mSUGRA model, the lower mass limit of the neutralino is found at $50 \mathrm{GeV}$. For large slepton and sneutrino masses there is a lower chargino mass limit of $m_{\widetilde{\chi}_{1}^{ \pm}}>103 \mathrm{GeV}$ from direct searches [21]. The lower mass limits for the sleptons are $99 \mathrm{GeV}, 94 \mathrm{GeV}$ and $86 \mathrm{GeV}$ for the selectron, smuon and stau respectively. These limits are derived from direct slepton searches with large mass differences between the slepton and the neutralino [22].

Several combinations of the mSUGRA parameters ${ }^{1}$, called SUSY points in the following, are generated to probe the region of the parameter space discussed in section 2.5.4. The SUSY points have different chargino and slepton masses in order to calculate the sensitivity for the SUSY signal $q \bar{q} \rightarrow \widetilde{\chi}_{1}^{ \pm} \widetilde{\chi}_{2}^{0} \rightarrow 3 \ell+2 \widetilde{\chi}_{1}^{0}+v$ with three charged leptons of any flavor in the final state. The SUSY points are chosen from the mSUGRA model but the results obtained are valid for any MSSM model with comparable masses and couplings at the electroweak scale.

Table 4.1 lists the SUSY points, along with the mSUGRA parameters and the masses of neutralinos, charginos and sleptons and the number of generated events. The chargino mass ranges from $100 \mathrm{GeV}<m_{\widetilde{\chi}_{1}^{ \pm}}<130 \mathrm{GeV}$, mostly beyond the LEP2 sensitivity of chargino searches. For each chargino mass, the selectron and smuon mass is varied from $m_{\tilde{\chi}_{1}^{ \pm}}-7 \mathrm{GeV}<m_{\tilde{\ell}}<m_{\tilde{\chi}_{1}^{ \pm}}+16 \mathrm{GeV}$. One point with $m_{\tilde{\ell}}>m_{\tilde{\chi}^{ \pm}}+100 \mathrm{GeV}$ has been added for each chargino mass as a reference for large slepton masses. The value of $\tan \beta=3$ is chosen to minimize the mixing in the stau sector and include the most sensitive region of the investigated decay channel and the value of the trilinear coupling is $\mathrm{A}_{0}=0$ for all SUSY points.

The expression $\sigma \times B R \cdot K_{q c d}$ in table 4.1 corresponds to the cross section for final states of the $\widetilde{\chi}_{1}^{ \pm} \widetilde{\chi}_{2}^{0}$ leptonic decays with any combination of three charged leptons. The factor $K_{q c d}$ is the next-to-leading order (NLO) correction factor for the cross section [27]. Its range is $1.23<K_{q c d}<1.26$ for the processes with the gaugino masses investigated. The corrected cross section will be referred to as $\sigma \times B R(3 \ell)$ in the following.

The SUSY points are labeled from A-F with increasing chargino mass. For each chargino mass the scan for $m_{0}$ has been labeled from 1-6. The corresponding masses of the neutralino $\left(\widetilde{\chi}_{1}^{0}\right)$ and the number of generated events are shown in the last two columns of table 4.1. In the following the SUSY points are referred to by their label i.e. SUSY

\footnotetext{
${ }^{1}$ At the GUT scale the mSUGRA parameters are (see also section 2.4.2): a common fermion mass $m_{1 / 2}$, a common scalar mass $m_{0}$, the ratio of the vacuum expectation values of the two Higgs fields $\tan \beta$, the trilinear coupling $A_{0}$ and the Higgs(ino) mass parameter $\mu$, with $|\mu|$ being fixed in mSUGRA.
} 


\begin{tabular}{|c|c|c|c|c|c|c|c|c|c|c|}
\hline \multicolumn{11}{|c|}{ SUSY signal processes } \\
\hline & $\begin{array}{c}m_{0} \\
{[\mathrm{GeV}]}\end{array}$ & $\begin{array}{c}m_{1 / 2} \\
{[\mathrm{GeV}]}\end{array}$ & $\mu$ & $\begin{array}{c}m_{\tilde{\chi}_{2}^{0}} \\
{[\mathrm{GeV}]}\end{array}$ & $\begin{array}{c}m_{\widetilde{\chi}_{1}^{ \pm}} \\
{[\mathrm{GeV}]}\end{array}$ & $\begin{array}{c}m_{\tilde{\ell}} \\
{[\mathrm{GeV}]}\end{array}$ & $\begin{array}{c}m_{\tilde{\tau}} \\
{[\mathrm{GeV}]}\end{array}$ & $\begin{array}{c}m_{\widetilde{\chi}_{1}^{0}} \\
{[\mathrm{GeV}]}\end{array}$ & $\begin{array}{c}\sigma \times B R \cdot K_{q c d} \\
{[\mathrm{pb}]}\end{array}$ & \# Events \\
\hline A1 & 56 & 165 & + & 102 & 98 & 92 & 90 & 55 & 1.03 & 20000 \\
\hline A2 & 64 & 165 & + & 102 & 98 & 97 & 95 & 55 & 0.77 & 21500 \\
\hline A3 & 72 & 175 & + & 102 & 98 & 102 & 101 & 55 & 0.39 & 17500 \\
\hline A4 & 80 & 175 & + & 102 & 98 & 108 & 106 & 55 & 0.35 & 18000 \\
\hline A5 & 88 & 175 & + & 102 & 98 & 114 & 112 & 55 & 0.29 & 18000 \\
\hline A6 & 200 & 126 & - & 102 & 103 & 208 & 207 & 52 & 0.06 & 22500 \\
\hline B1 & 60 & 170 & + & 106 & 101 & 96 & 94 & 58 & 0.86 & 18000 \\
\hline B2 & 68 & 170 & + & 106 & 101 & 101 & 99 & 58 & 0.66 & 20000 \\
\hline B3 & 76 & 170 & + & 106 & 101 & 106 & 105 & 58 & 0.32 & 25700 \\
\hline B4 & 84 & 170 & + & 106 & 101 & 112 & 110 & 58 & 0.28 & 21000 \\
\hline B5 & 92 & 170 & + & 106 & 101 & 118 & 117 & 58 & 0.24 & 21000 \\
\hline B6 & 200 & 132 & - & 106 & 107 & 209 & 207 & 54 & 0.05 & 22500 \\
\hline $\mathrm{C} 1$ & 64 & 175 & + & 110 & 106 & 99 & 98 & 60 & 0.73 & 21500 \\
\hline $\mathrm{C} 2$ & 72 & 175 & + & 110 & 106 & 105 & 103 & 60 & 0.55 & 22000 \\
\hline C3 & 80 & 175 & + & 110 & 106 & 110 & 109 & 60 & 0.26 & 23900 \\
\hline $\mathrm{C} 4$ & 88 & 175 & + & 110 & 106 & 116 & 115 & 60 & 0.23 & 23500 \\
\hline $\mathrm{C} 5$ & 96 & 175 & + & 110 & 106 & 122 & 121 & 60 & 0.19 & 24500 \\
\hline C6 & 200 & 138 & - & 110 & 111 & 210 & 208 & 57 & 0.04 & 19500 \\
\hline D1 & 68 & 180 & + & 114 & 110 & 103 & 101 & 62 & 0.60 & 25500 \\
\hline D2 & 76 & 180 & + & 114 & 110 & 109 & 107 & 62 & 0.47 & 21500 \\
\hline D3 & 84 & 180 & + & 114 & 110 & 114 & 113 & 62 & 0.21 & 25400 \\
\hline D4 & 92 & 180 & + & 114 & 110 & 120 & 119 & 62 & 0.19 & 21500 \\
\hline D5 & 100 & 180 & + & 114 & 110 & 126 & 125 & 62 & 0.15 & 23000 \\
\hline D6 & 200 & 142 & - & 114 & 114 & 210 & 208 & 58 & 0.04 & 21500 \\
\hline E1 & 72 & 185 & + & 118 & 114 & 107 & 105 & 65 & 0.51 & 31000 \\
\hline E2 & 80 & 185 & + & 118 & 114 & 112 & 111 & 65 & 0.40 & 21500 \\
\hline E3 & 88 & 185 & + & 118 & 114 & 118 & 117 & 65 & 0.18 & 22000 \\
\hline E4 & 96 & 185 & + & 118 & 114 & 124 & 123 & 65 & 0.15 & 22000 \\
\hline E5 & 104 & 185 & + & 118 & 114 & 130 & 129 & 65 & 0.13 & 37000 \\
\hline E6 & 200 & 148 & - & 118 & 119 & 211 & 209 & 61 & 0.03 & 20500 \\
\hline $\mathrm{F} 1$ & 90 & 205 & + & 135 & 132 & 124 & 123 & 74 & 0.26 & 14000 \\
\hline $\mathrm{F} 2$ & 98 & 205 & + & 135 & 132 & 130 & 129 & 74 & 0.18 & 19000 \\
\hline F3 & 106 & 205 & + & 135 & 132 & 136 & 135 & 74 & 0.07 & 18000 \\
\hline $\mathrm{F} 4$ & 114 & 205 & + & 135 & 132 & 142 & 141 & 74 & 0.07 & 15500 \\
\hline F5 & 122 & 205 & + & 135 & 132 & 148 & 147 & 74 & 0.06 & 28000 \\
\hline F6 & 200 & 166 & - & 132 & 132 & 212 & 211 & 69 & 0.02 & 18500 \\
\hline
\end{tabular}

Table 4.1: SUSY signal process $q \bar{q} \rightarrow \widetilde{\chi}_{1}^{ \pm} \widetilde{\chi}_{2}^{0} \rightarrow 3 \ell+2 \widetilde{\chi}_{1}^{0}+v$ for $\tan \beta=3, A_{0}=0$. 
point A1.

\subsubsection{Standard Model Backgrounds}

The final state of the SUSY signal under investigation $\widetilde{\chi}_{1}^{ \pm} \widetilde{\chi}_{2}^{0} \rightarrow \mu \mu \ell+\mathbb{E}_{T}$ is characterized by three isolated leptons. The Standard Model background processes can also have isolated muons or leptons in the final state. Assuming that the leptonic decays of the $Z \rightarrow \mu^{+} \mu^{-}$production can be identified with high efficiency, the instrumental backgrounds to the SUSY signal are expected to be small.

Standard Model processes with two isolated muons in the final state are background for the SUSY signal, if an additional lepton from a jet is identified as the third muon, electron or tau ${ }^{2}$. The Monte Carlo samples of the Standard Model processes considered as backgrounds to the SUSY signal are listed in table 4.2 along with the associated cross section, with the higher order corrections applied, times branching fraction and the number of generated events.

\begin{tabular}{lrrr}
\hline \hline Background Process & & $\sigma \times$ BR [pb] & \# Events \\
\hline$W Z \rightarrow \mu^{+} v \mu^{-} \mu^{+}$ & & 0.0064 & 5250 \\
$W Z \rightarrow e^{+} \vee \mu^{-} \mu^{+}$ & & 0.0064 & 2500 \\
$Z / \gamma^{*} \rightarrow \mu^{+} \mu^{-}$ & $\left(5<M_{\mu \mu}<15\right) \mathrm{GeV}$ & 4565 & 219250 \\
$Z / \gamma^{*} \rightarrow \mu^{+} \mu^{-}$ & $\left(15<M_{\mu \mu}<60\right) \mathrm{GeV}$ & 434 & 432000 \\
$Z / \gamma^{*} \rightarrow \mu^{+} \mu^{-}$ & $\left(60<M_{\mu \mu}<130\right) \mathrm{GeV}$ & 254 & 665340 \\
$Z / \gamma^{*} \rightarrow \mu^{+} \mu^{-}$ & $\left(130<M_{\mu \mu}<250\right) \mathrm{GeV}$ & 2 & 10000 \\
$Z / \gamma^{*} \rightarrow \tau^{+} \tau^{-}$ & $\left(5<M_{\tau \tau}<15\right) \mathrm{GeV}$ & 4565 & 301250 \\
$Z / \gamma^{*} \rightarrow \tau^{+} \tau^{-}$ & $\left(15<M_{\tau \tau}<60\right) \mathrm{GeV}$ & 434 & 644313 \\
$Z / \gamma^{*} \rightarrow \tau^{+} \tau^{-}$ & $\left(60<M_{\tau \tau}<130\right) \mathrm{GeV}$ & 254 & 655000 \\
$Z / \gamma^{*} \rightarrow \tau^{+} \tau^{-}$ & $\left(130<M_{\tau \tau}<250\right) \mathrm{GeV}$ & 2 & 114250 \\
$W W \rightarrow \mu^{+} v \mu^{-} \bar{v}$ & & 0.15 & 20750 \\
$t \bar{t} \rightarrow \mu^{ \pm} v \mu^{ \pm} v b \bar{b}$ & & 0.07 & 39000 \\
$Z b \bar{b} \rightarrow \mu^{ \pm} \mu^{ \pm}+b \bar{b}$ & & 0.61 & 96500 \\
\hline$W \rightarrow \mu^{ \pm} v$ & & 2695 & 1640550 \\
$W b \bar{b} \rightarrow \mu^{ \pm} v+b \bar{b}$ & & 1.92 & 198500 \\
\hline
\end{tabular}

Table 4.2: Standard Model background processes classified by their final state signatures, which can have two or one real isolated muons in the event. $M_{\mu \mu}\left(M_{\tau \tau}\right)$ is the reconstructed invariant mass of the dimuon(ditau) pair.

The $W Z$ backgrounds have three isolated leptons in the final state arising from the leptonic decays of the electroweak gauge bosons. The kinematic properties of these background events are similar to the ones of the SUSY signal in the final state. Nevertheless the cross section is very small, therefore this irreducible background can be neglected.

\footnotetext{
${ }^{2}$ The criterion of a third lepton is replaced by the requirement of a third isolated charged track in the later analysis.
} 
The Drell-Yan backgrounds $Z / \gamma^{*} \rightarrow \mu^{+} \mu^{-}$and $Z / \gamma^{*} \rightarrow \tau^{+} \tau^{-}$have events with two isolated muons in the final state. A third lepton (if classified) comes from a misidentified track, isolated pions or tracks from converted photons in the event. The $W W$ production leads to final states with two muons from the semi-leptonic decay of the $\mathrm{W}$ bosons. The semi-leptonic decays from the $t \bar{t}$ production leads to final states with isolated muons. In the case of the $Z b \bar{b}$ background, the leptons are produced in the cascade decay of the $Z$ boson and additional fake leptons are selected from the misidentification of isolated hadrons as leptons, or leptons from the heavy flavor decays. This background is already included in the inclusive $Z / \gamma^{*}$ decay and is added for describing the Monte Carlo properly. Its cross section is small and it is completely reduced after the first selection cuts. Therefore a potential double counting can be excluded.

One muon in the $W$ background events originates from the leptonic decay of the W boson. The second muon and the additional lepton are selected from semi-leptonic heavy flavor decay that can yield muons and tracks which are then classified as leptons or isolated tracks. The $W b \bar{b}$ background was added to the $\mathrm{W}$ background in order to describe the Monte Carlo properly. The cross section is small and potential double counting can be excluded.

The next-to-next-to-leading order corrections (NNLO) of the cross sections of the $Z / \gamma^{*}$ backgrounds and the next-to-leading order (NLO) corrections of the cross sections of $W W$ and $W Z$ production are taken from [42]. For the processes $t \bar{t}$ the cross section is taken from [43]. The processes $W b \bar{b}$ and $Z b \bar{b}$ are generated using ALPGEN 1.2 [44] and PythiA 6.2 [35]. The NLO cross sections are given as described in [45, 46].

An additional background arises from heavy flavor production, mostly from semileptonic decays of $b$ and $c$ quarks. This background is estimated from data and is therefore not listed in table 4.2. A detailed description of this QCD background estimation can be found in section 4.6.

\subsection{Data Samples}

The data sample used in this analysis was collected with the DØ detector from August 2002 to June 2004. During this period almost 1 billion events have been recorded and 25 skims ${ }^{3}$ have been written out. The skim used for this analysis is called dimuon skim and represents $4.7 \%$ of the whole dataset corresponding to 1.3 TByte. Events with at least two loose muons (a loose muon requires a certain hit pattern in the muon system) and without any requirement for the transverse momentum of the muon were selected from data.

A run quality selection has been applied using the classifications from the run quality database of $\mathrm{D} \varnothing[48]$. When inquiring the database, all runs marked as bad for the muon system, the calorimeter or the missing transverse energy or the central fiber tracking and the silicon microstrip tracker are rejected. In addition, run ranges where the dimuon trigger system was not working properly have been excluded.

\footnotetext{
${ }^{3}$ A skim is a preselection of data events containing specific physics objects. The skimming code runs at a speed of approximately $0.3 \mathrm{~s} / \mathrm{event}[47]$.
} 


\subsubsection{Trigger Selection and Efficiency}

The rate of $p \bar{p}$ collisions at the Tevatron is significantly higher than the rate at which events can be read out and stored for offline analysis. Therefore, events are selected online with event triggers (a detailed description of the trigger system at $\mathrm{D} \emptyset$ is given in section 3.2.4).

The choice of the event triggers defines the final dataset. For the presented analysis all data events, with at least two muons (also called dimuon events) are of interest. Data events are selected online if accepted by at least one of the following dimuon triggers: 2MU_A_L2M0,2MU_A_L2ETAPHI, 2MU_A_L2M0_TRK10, 2MU_A_L2M0_TRK5 [49]. These triggers are combined with a logical OR as they can trigger a given data event simultaneously.

Each dimuon trigger is a combination of muon scintillating triggers at Level 1 and a medium muon ${ }^{4}$ trigger at Level 2. The last two triggers also require a track at Level 3 with transverse momentum of $p_{T}>10 \mathrm{GeV}$ or $p_{T}>5 \mathrm{GeV}$. A more detailed description of the muon trigger is given in section 3.2.5.

The trigger efficiency for events selected by the dimuon trigger 2MU_A_L2MO is determined to be $\varepsilon=0.76 \pm 0.02$ [50]. The dataset triggered by 2MU_A_L2ETAPHI alone is only about $5 \%$ of the whole dataset. Therefore the trigger efficiency is assumed to be unchanged within errors. The corresponding dataset for events accepted only by the trigger 2MU_A_L2M0_TRK5 is $31 \%$ of the whole dataset. The inefficiency of this trigger with respect to 2MU_A_L2M0 has been taken into account by studying datasets for which both triggers accepted the same event. The L3 efficiency for trigger 2MU_A_L2M0_TRK5 has been calculated to be 79\%. Then, the trigger efficiency for the trigger 2MU_A_L2M0 is corrected with $\varepsilon=0.94$ [51]. Trigger 2MU_A_L2M0_TRK10 was prescaled (see section 3.2.4 for prescale definition) for most of the events considered and not taken into account. Nevertheless few events are selected only by this trigger when it was not prescaled.

\subsubsection{Integrated Luminosity}

The integrated luminosity of the data sample is calculated using the software package $1 m \_$access_pkg provided by the luminosity ID group [52]. The integrated luminosity for the data sample is calculated for each of the selected triggers separately. Only runs where at least one of the selected triggers has no prescale and events accepted by at least one of the triggers are taken into account. Table 4.3 shows the integrated luminosity for the whole data sample and for each trigger separately.

The trigger 2MU_A_L2M0 was used from the very beginning. The accelerator luminosities increased in July 2003 and the first two triggers had to be prescaled when the trigger rates increased. The total integrated luminosity for the data sample is $\mathcal{L} \sim 300 \mathrm{pb}^{-1}$.

\footnotetext{
${ }^{4}$ see 4.5 .1 for medium muon definition
} 


\begin{tabular}{c|c}
\hline \hline Trigger name & $\mathcal{L}\left[\mathrm{pb}^{-1}\right]$ \\
\hline 2MU_A_L2M0 & 182.6 \\
2MU_A_L2ETAPHI & 172.8 \\
2MU_A_L2M0_TRK10 & 61.6 \\
2MU_A_L2M0_TRK5 & 189.3 \\
\hline OR of all triggers & 299.5 \\
\hline
\end{tabular}

Table 4.3: Integrated luminosity $\mathcal{L}$ of the data sample.

\subsection{Monte Carlo Normalization to Data}

The Monte Carlo siulation samples are normalized to the integrated luminosity of the data sample. In order to assure that the data events are described correctly by the Monte Carlo simulation, a well-known process is examined: $Z / \gamma^{*} \rightarrow \mu^{+} \mu^{-}$. In the mass range of $60 \mathrm{GeV}<\mathrm{M}_{\mu \mu}<120 \mathrm{GeV}$ the relation $\mathrm{N}_{\text {DATA }}^{\mathrm{Z} / \gamma^{*}}=\mathrm{f} \cdot \mathrm{N}_{\mathrm{MC} \text {,exp }}^{\mathrm{Z} / \gamma^{*}}$ should be satisfied, where $\mathrm{N}_{\text {DATA }}^{\mathrm{Z} / \gamma^{*}}$ is the number of data events and $\mathrm{N}_{\text {MC,exp }}^{\mathrm{Z} \gamma^{*}}$ is the number of expected Monte Carlo events, taking into account the Monte Carlo reconstruction efficiency and luminosity. The scale factor $\mathrm{f}$ takes into account the difference in efficiencies between data and Monte Carlo and the trigger efficiency. Its value is $\mathrm{f}=0.53 \pm 0.03$ and has been applied to all Monte Carlo processes. Figure 4.1 shows the comparison of the data and Monte Carlo

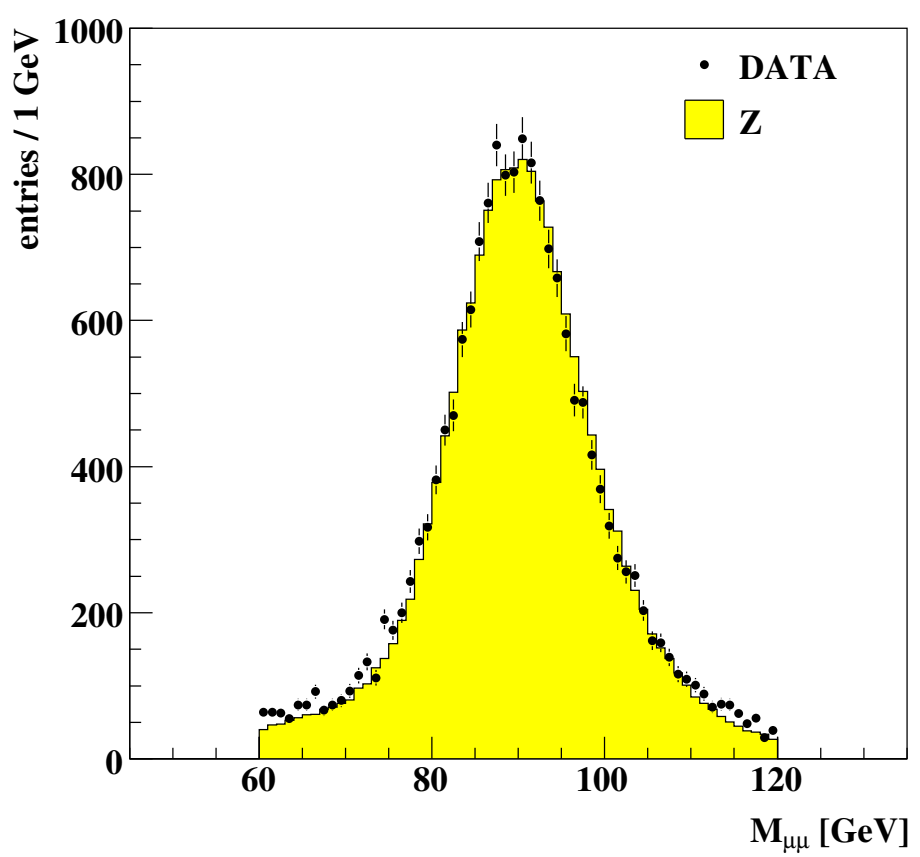

Figure 4.1: The $\mathrm{Z}$ resonance from Monte Carlo events (yellow filled histogram) is normalized to data (black dots). The Monte Carlo is smeared according to equation (4.1). 
events after normalization. The transverse momenta of the muons in the Monte Carlo sample are smeared as will be shown in section 4.5.2.

It has been shown in [50], that the normalization of the Monte Carlo to the data taking into account the detector acceptance, trigger efficiency and Monte Carlo reconstruction efficiencies gives the same scaling factor for the process $Z / \gamma^{*} \rightarrow \mu^{+} \mu^{-}$as adjusting the Monte Carlo to the data in the range of the $Z$ resonance. The product of the trigger efficiency and the differences of the efficiencies between data and Monte Carlo has been determined to be $0.6 \pm 0.04$. Taking into account the L3 inefficiency of the trigger 2MU_A_L2M0_TRK5 this predicts an overall scaling factor of $0.56 \pm 0.04$ which is in good agreement with the scaling factor described above.

\subsection{Event Reconstruction and Physics Objects}

The triggers select online those data events, that are written to tape for further analysis. The particle trajectories and momenta in the event as well as their energy deposition in the calorimeter are then reconstructed offline. The reconstructed event is then characterized by its physics objects which are muons, tracks, jets and missing transverse energy. Also the simulated events are reconstructed from the digitised output at the end of the simulation chain. The event reconstruction program for either data or simulated events runs on dedicated computer farms, like GridKa in Karlsruhe.

The physics objects are obtained using the standard event reconstruction software of DØ (DØReco) [53]: p14.02.00, p14.03.02, p14.05.02 and p14.06.01. The output of the reconstruction program are the ThumbNail files. The ThumbNail data format is discussed in more detail in appendix A.

Trajectories (tracks) of particles are reconstructed from the hits left during their passage in the tracking detectors. Tracks are extrapolated back towards the nominal beam position, with any point of overlap forming a potential vertex. A momentum-weighted sum of the tracks meeting at the vertex is used to select the primary (collision) vertex. If no primary vertex is reconstructed, the default position in the geometrical center of the detector is taken.

Reconstruction of jets (see section 4.5.3 for detailed definition) in the calorimeter is based on clusters of neighboring cells containing energy deposits of the tracks. Starting from a cluster, a cone with an opening angle of 0.5 originating at the primary vertex is defined in $\eta-\phi$. The total energy within this cone is summed up including the fraction in the electromagnetic and hadronic layers which are used to separate the electromagnetic and hadronic showers. The energy distribution in the calorimeter is also used to identify the missing transverse energy in events (see section 4.5.3). This missing transverse energy must be corrected for the presence of any muons in the event, as muons deposit only a fraction of their energy in the calorimeter.

The reconstruction of muons is done using two detector components: the muon system itself and the tracking detectors. First a muon track is reconstructed in the muon system and classified by its quality into loose, medium and tight (section 4.5.1). 


\subsubsection{Muon Particles: Identification and Efficiency}

Muon tracks are reconstructed in the muon system and matched to tracks in the central tracking system. A simultaneous 'global' fit of all hits along the muon trajectory yields the muon particle which provides all muon track information used in the further analysis.

Muon tracks are identified by the muon system from hits in wire chambers and scintillation counters. The muon tracks are classified according to their track quality information as tight, medium or loose. A muon track is classified as 'tight' if the track is reconstructed from at least two hits in the wire chambers in the A layer, at least one hit in the A layer scintillator counters, at least three hits in the BC layer wire chambers, at least one hit in the $\mathrm{BC}$ layer scintillator counters, and a converged fit through the hits of the A and BC layers of the muon system. A muon track is classified as 'medium' if it has at least two hits in the A layer wire chambers, at least one hit in the A layer scintillator counters, at least two hits in the $\mathrm{BC}$ layer wire chambers and at least one hit in the scintillator counters of the BC layer. A 'loose' muon track is defined as a medium muon track but allowing one of the above tests to fail, with the A layer wire chamber and scintillator counters requirement treated as one test and requiring always at least one hit in the scintillator counters.

Muons detected in the muon system and matched to tracks with no hits in the SMT, are extrapolated towards the reconstructed vertex, and the transverse momentum of these tracks is corrected with respect to this vertex. All identified muons satisfy the certification criteria obtained by the muon ID group [54].

Cosmic muons can equally pass any of the above trigger criteria. They can however be distinguished from muons of real collisions and can be rejected by requiring the time difference between bunch crossing and scintillation counters coincidence to be below 10 ns. Furthermore, the distance of closest approach $(d c a)$ of the muon, in the coordinates $\mathrm{r}-\phi$, to the reconstructed vertex of the $p \bar{p}$ collision is required to be less than $0.15 \mathrm{~cm} \mathrm{[54]}$.

Events with two loose muons, each matched to one central track, are retained for further analysis. The transverse momenta for the two leading $p_{T}$ muons in selected events is $p_{T}^{\mu_{1}}>9.0 \mathrm{GeV}$ for the first muon and $p_{T}^{\mu_{2}}>5.0 \mathrm{GeV}$ for the second muon. The selected muons must be isolated in a cone of $\Delta R=\sqrt{(\Delta \phi)^{2}+(\Delta \eta)^{2}}<0.5$ with respect to all other tracks, where $\Delta \phi$ is the azimuthal angle and $\Delta \eta$ is the difference of the pseudo-rapidity of the tracks. Muons are minimum ionizing particles in the calorimeter, therefore the energy deposited by the muon in the calorimeter cells should be small.

The isolation criteria for both muons in the event are as:

- the sum of the $p_{T}$ of all other tracks in the cone is less than $\Sigma_{\text {tracks }}^{\Delta R<0.5} p_{T}<4.0 \mathrm{GeV}$

- at least one of the muons must have a tighter isolation; the energy deposited in the calorimeter cells in a hollow cone around the muon track is less than $\Sigma_{\text {cells }}^{0.1<\Delta R<0.4} E_{T}<2.5 \mathrm{GeV}$ and the sum of all $p_{T}$ of tracks is $\Sigma_{\text {tracks }}^{\Delta R<0.5} p_{T}<2.5 \mathrm{GeV}$.

In addition both selected muons are required to be from the same reconstructed vertex within $1 \mathrm{~cm}$. The vertex should be identical to the primary vertex within $2 \mathrm{~cm}$. 


\section{Muon Tracking Efficiency}

The tracking efficiency is calculated using the $Z \rightarrow \mu^{+} \mu^{-}$decay for data and Monte Carlo events. The reconstructed invariant mass of the muons is $60<M_{\mu \mu}<120 \mathrm{GeV}$. Muons have a transverse momentum $p_{T}>15 \mathrm{GeV}$ (determined by the muon system alone). To obtain a clean selection, both muons are required to be separated by $\Delta \phi>2.5$ and must be isolated within $\Sigma_{\text {cells }}^{0.1<\Delta R<0.4} E_{T}<2.5 \mathrm{GeV}$. The selected events must be triggered by one of the triggers described above. The tracking efficiency for data and Monte Carlo is $\varepsilon_{\text {track,DATA }}=0.928 \pm 0.008$ (stat.) and $\varepsilon_{\text {track,MC }}=0.961 \pm 0.005$ (stat.) [55]. The tracking efficiency for the Monte Carlo simulation is higher than for the data, due to the overestimated hit efficiency.

\section{Muon Reconstruction Efficiency}

The efficiency to reconstruct a loose muon in the $\mathrm{D} \emptyset$ muon system is calculated using pairs of muons with a reconstructed invariant mass of $60 \mathrm{GeV}<M_{\mu \mu}<120 \mathrm{GeV}$ for data and Monte Carlo events. One muon must be matched to a track with transverse momentum $p_{T}>20 \mathrm{GeV}$ and be of loose muon quality. The second muon is also matched to a track with $p_{T}>10 \mathrm{GeV}$ and tested if its quality is loose. The test-muon is a calorimeter muon, and therefore must not necessarily be detected by the muon chambers. Both muons are required to be separated in $\Delta \phi>2.5$. The selected events must be triggered by one of the triggers described above. The loose muon reconstruction efficiency excluding the acceptance gap at the bottom of the detector, for $4.25<\phi<5.15$, is found to be $\varepsilon_{\text {loose,DATA }}=0.91 \pm 0.01$ (stat.) for data and $\varepsilon_{\text {loose }, \mathrm{MC}}=0.90 \pm 0.01$ (stat.) for Monte Carlo [55].

\section{Muon Isolation Efficiency}

The efficiency for isolated muons is calculated for pairs of muons from $Z \rightarrow \mu^{+} \mu^{-}$decays for data and Monte Carlo events. The reconstructed invariant mass must be in a mass window of $70 \mathrm{GeV}<M_{\mu \mu}<110 \mathrm{GeV}$. Both muons are matched to central tracks with transverse momenta $p_{T}>15 \mathrm{GeV}$ and separated in $\Delta \phi>2.5$. One muon must fulfill the isolation criteria described above. The second muon is tested whether it satisfies the isolation criteria. The selected events must be triggered by one of the triggers described above. The muon isolation efficiency is then $\varepsilon_{\text {iso,DATA }}=0.97 \pm 0.01$ (stat.) for data and $\varepsilon_{\text {iso,MC }}=0.98 \pm 0.01$ (stat.) for Monte Carlo [55].

\section{Remaining efficiencies}

The efficiency for charge mismeasurement is $\varepsilon_{\mathrm{q}, \mathrm{DATA}}=0.99 \pm 0.01$ (stat.) [56]. It is determined from the number of events rejected by the selection of same-sign muon pairs in the invariant mass range of the $Z$ resonance. Cosmic muons are rejected applying the standard requirements recommended by the muon-ID group [54] on the scintillator times. The rejection efficiency for cosmic muons $\varepsilon_{\text {cosmic,DATA }}=0.995 \pm 0.005$ (stat.) is obtained 
by selecting acolinear tracks with high scintillator times and the geometrical acceptance is $\varepsilon_{\mathrm{acc}}=0.42 \pm 0.01[56]$.

\subsubsection{Muon Momentum Smearing for Monte Carlo Samples}

The width of the $\mathrm{Z}$ resonance distribution expected from Monte Carlo $Z \rightarrow \mu^{+} \mu^{-}$events, is smaller than the one for data events due to wrong $p_{T}$ distributions. Figure 4.2 a) shows the dimuon mass $\left(M_{\mu \mu}\right)$ for data and Monte Carlo events (the Monte Carlo events are normalized to the data). The muons are selected according to the identification and isolation criteria described in section 4.5.1. The distribution of the transverse momenta for both muons $\left(p_{T}^{\mu}\right)$ is shown in figure $4.2 \mathrm{~b}$ ). From this, it is obvious that the default Monte Carlo must be adjusted to describe the data properly. To achieve that, the transverse momentum
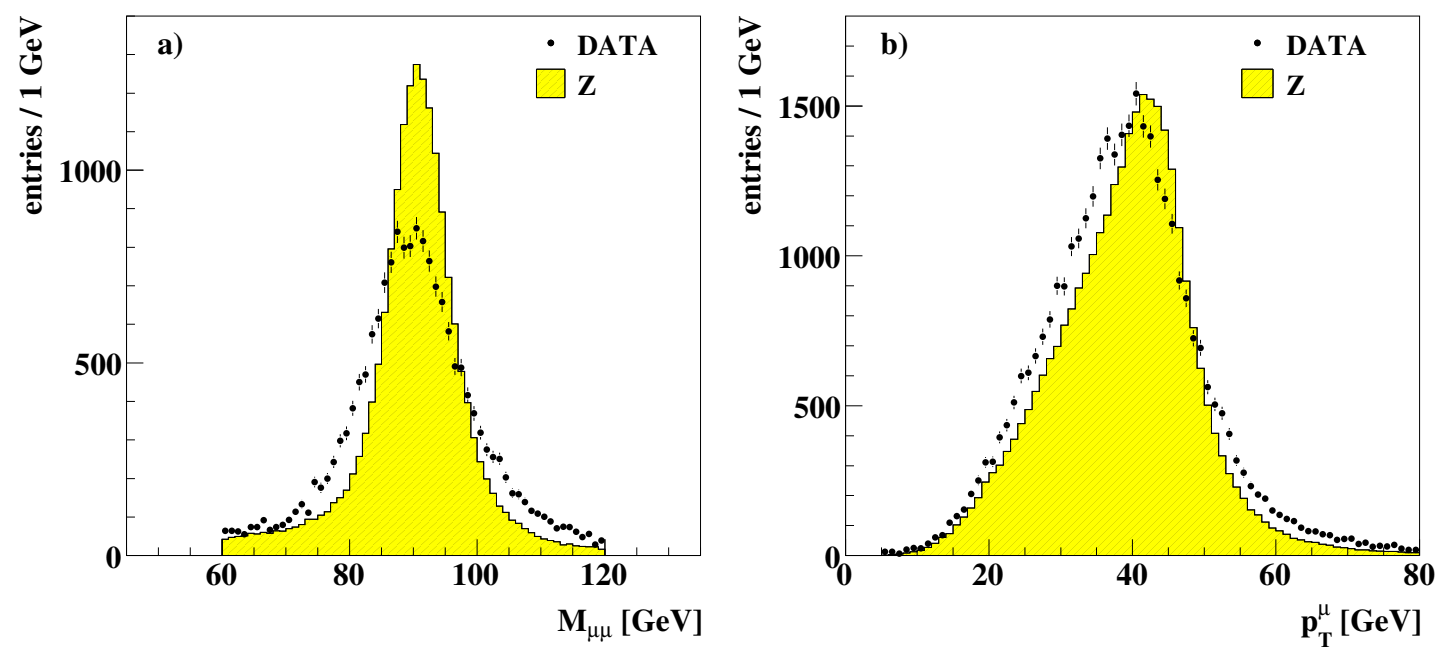

Figure 4.2: The dimuon mass (left) and the transverse momenta of the two muons (right), for the $Z \rightarrow \mu^{+} \mu^{-}$Monte Carlo events (yellow filled histogram) compared to data events (black dots).

of the muon is smeared using the following function [51, 54]:

$$
\frac{1}{p_{T}} \rightarrow \frac{1.005}{p_{T}}+\left(A+\frac{B}{p_{T}}\right) \cdot G
$$

where $G$ is a random variable drawn from a Gaussian distribution with width 1 and mean 0 . The parameters $A$ and $B$ are determined from a fit to the data distribution. For muons with at least one SMT hit $A$ and $B$ are returned as 0.0018 and 0.017 , respectively, while for muons with no SMT hits $A$ is 0.0023 and $B$ is 0.028 .

After smearing the transverse momentum of the muons, the data is described correctly. This is shown in figure $4.3 \mathrm{a}, \mathrm{b}$ ) for the dimuon mass and the transverse momentum for both muons. The smearing method described by equation (4.1), is applied to all muons for all Monte Carlo samples. In the later analysis, also isolated tracks (if selected) will be smeared according to the equation. 

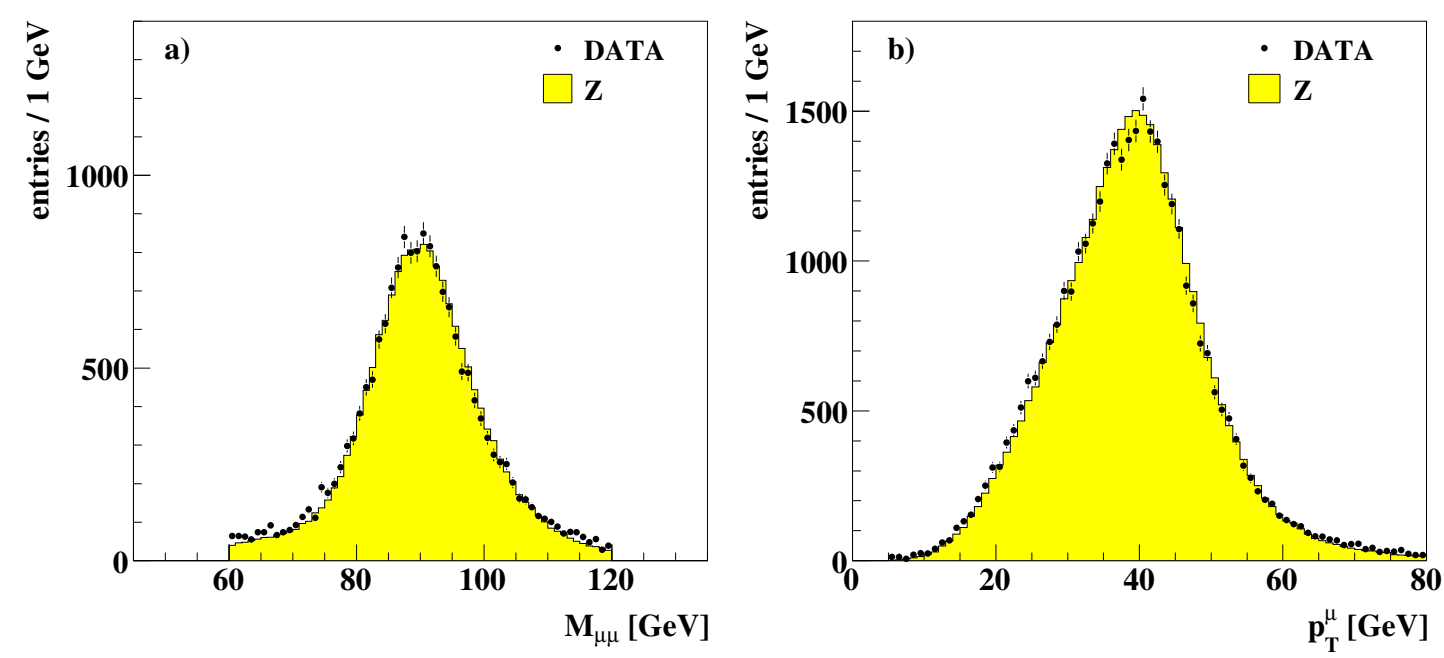

Figure 4.3: The reconstructed invariant mass (left) and the transverse momentum for both muons (right) after smearing. The $Z \rightarrow \mu^{+} \mu^{-}$Monte Carlo events are shown as the yellow filled histogram. Data events are plotted as black dots.

\subsubsection{Jets and Missing Transverse Energy $\left(\mathscr{E}_{T}\right)$}

\section{Jets}

The particle jets are reconstructed by measuring the jet energy in the calorimeter cells and towers. A jet algorithm associates clusters of the particles into jets. The kinematic properties of the jets can then be related to the corresponding properties of the energetic partons produced in the hard scattering process. The algorithm selects a set of particles which are emitted close to each other and combines their momenta to form the momentum of the jet. At DØ an iterative cone algorithm is used [57].

A jet cone of radius $R$ contains all particles with trajectories in an area $A=\pi R^{2}$. The radius of the jet is defined as $R=\sqrt{(\Delta \phi)^{2}+(\Delta \eta)^{2}}=0.5$, where $\eta$ is the pseudorapidity and $\phi$ is the azimuthal angle. The axis of the cone defines the direction of the jet. If HOTf is the ratio of the energy of the highest to the next-to-highest calorimeter tower, CHF is the energy fraction deposited in the coarse hadronic layer of the calorimeter, N90 is the number of calorimeter towers containing $90 \%$ of the energy of a jet and EMf is the fraction of the jet energy deposited in the electromagnetic layers of the calorimeter, then jets are reconstructed as good jets if they satisfy the following quality criteria:

$$
\text { HOTf }<10, \quad \mathrm{CHF}<0.4, \quad \mathrm{~N} 90>1, \quad \text { EMf }>0.05 .
$$

Additionally, the ratio between the jet energy measured by the L1 system and the jet energy measured by the precision readout, must be larger than 0.4 [58]. 


\section{Jet Energy Scale Correction}

The measured energy of the jet depends on the migrations into and out of the jet cone, the imperfections of the energy measurements in the calorimeter and the underlying event energy within the cone of the jet. The limited jet cone size results in energy flows outside the jet boundary because of showering in the calorimeter. Therefore, the dominant part of the jet energy scale uncertainty comes from the jet response of the calorimeter.

The jet energies are studied in $\gamma+$ jet $(s)$ events, where the highly energetic photon it is well contained in a few calorimeter cells. The photon recoils from the jet and allows a precise measurement of its energy. The conservation of transverse momentum then allows the correction of the jet energy in a cone of 0.5 [59].

\section{Missing Transverse Energy}

The missing transverse energy $\left(\mathbb{E}_{T}\right)$ in an event is a typical signature of neutralinos $\left(\widetilde{\chi}_{1}^{0}\right)$ or neutrinos, which escape detection. It has to be corrected for the other reconstructed objects in the event: electromagnetic objects, jets, muons and for the jet energy scale. The missing transverse energy is therefore the last object computed in the reconstruction.

Following the recommendations of the DØ Jet/Missing Energy ID group [60], the missing transverse energy is recalculated using all calorimeter cells with an energy above $0 \mathrm{MeV}$, where the unclustered energy in the coarse hadronic layers is excluded except for coarse hadronic cells which belong to a jet. Afterwards a jet energy scale correction is applied. The muon transverse momentum given by the tracking system is subtracted from the missing transverse energy corrected for all other reconstructed objects. The value of the energy loss of the muon in the calorimeter of about $2 \mathrm{GeV}$ (obtained from an empirical function from test beam data) is due to ionization and is added to the $\mathbb{E}_{T}$, taking into account any angular dependency. Only muons of at least loose quality, which can be matched to a central track and have a $d c a<0.15 \mathrm{~cm}$ with respect to the primary interaction vertex have been used. For Monte Carlo samples the missing transverse energy is corrected for the smeared transverse momenta of the muons described above.

\section{Scaled Missing Transverse Energy}

For background events, large values of missing transverse energy can be produced by the mismeasurement of the jet energy. Therefore, the significance $\operatorname{Sig}\left(\mathbb{E}_{T}\right)$ is defined by normalizing $\mathbb{E}_{T}$ to the uncertainty due to the resolution of the jets projected onto the $\mathbb{E}_{T}$ direction [61]:

$$
\operatorname{Sig}\left(\mathbb{E}_{T}\right)=\frac{\mathbb{E}_{T}}{\sqrt{\sum_{j e t s}\left(\sqrt{E_{j e t}} \cdot \sin \theta_{j e t} \cdot\left|\cos \left(\Delta \phi\left(j e t, \mathbb{E}_{T}\right)\right)\right|\right)^{2}}}
$$

where $E_{j e t}$ is the jet energy, $\theta_{j e t}$ is the polar angle of the jet and $\Delta \phi$ is the azimuthal angle between the jet direction and $\mathbb{E}_{T}$. The square-root in equation (4.2) is due to the fact that the energy resolution of the jet is proportional to the square-root of the jet energy. 


\subsection{QCD Background Estimation}

The QCD multi-jet background, in the further analysis referred to as QCD background, consists mainly of decays from $b \bar{b}$ events. In the detector $b(\bar{b})$ quarks are seen as parton showers that form jets. The $\mathrm{b}$ quarks can have semi-leptonic direct decays $b \rightarrow \mu$ or cascade decays $b \rightarrow c \rightarrow \mu$ into the final state with muons.

The production cross section for this background is fairly large. Therefore this process is not simulated with Monte Carlo samples but estimated from data [62, 63]. Fortunately the muons in QCD events are predominantly not isolated being in or in the vicinity of the $b(\bar{b})$ jet. The QCD background can therefore be estimated from a sample of data events with anti-isolated muons. Events with at least two anti-isolated muons are selected if the muons are anti-isolated with respect to other tracks in a cone of $\Delta R<0.5$ around the muon track defined as:

- the sum of the $p_{T}$ of all other tracks in the cone is greater than $\Sigma_{\text {tracks }}^{\Delta R<0.5} p_{T}>2.5 \mathrm{GeV}$ and the energy deposited in the calorimeter cells in a hollow cone around the muon track is greater than $\Sigma_{\text {cells }}^{0.1<\Delta R<0.4} E_{T}>2.5 \mathrm{GeV}$.

- one of the muons has a tighter anti-isolation $\Sigma_{\text {cells }}^{0.1<\Delta R<0.4} E_{T}>4.0 \mathrm{GeV}$.

The two leading $p_{T}$ muons in selected events pass the same transverse momenta requirements as in the case of events with isolated muons: $p_{T}^{\mu_{1}}>9.0 \mathrm{GeV}\left(p_{T}^{\mu_{2}}>5.0 \mathrm{GeV}\right)$ for the first (second) muon in the event.

The number of events from QCD processes with two isolated muons $\mathrm{N}_{\text {isolated }}^{\mathrm{QCD}}$ is then determined from the number of data events with two anti-isolated muons like

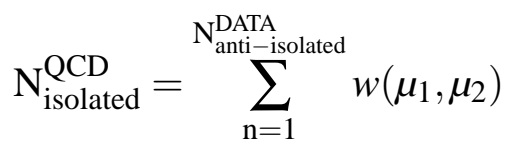

where $\mathrm{N}_{\text {anti-isolated }}^{\text {DATA }}$ is the number of events with at least two anti-isolated muons selected from data. The relating weight factor $w\left(\mu_{1}, \mu_{2}\right)$ is applied for each event. It depends on the transverse momentum of the muons $p_{T}^{\mu_{1}}$ and $p_{T}^{\mu_{2}}$, which is taken from Monte Carlo simulation. It further includes a normalization factor, taking into account the correlation between the muons, which is determined from data. Both calculation steps are described in the following.

\section{Calculation of the weight factor $w\left(\mu_{1}, \mu_{2}\right)$}

In a first step the QCD Monte Carlo sample is analyzed: the $b \bar{b}$ process was generated with PYTHIA [35] where the b quark is decaying using EvTGEN [64]. Only events with at least two muons with transverse momenta of $p_{T}^{\mu_{1}}>8 \mathrm{GeV}$ and $p_{T}^{\mu_{2}}>3 \mathrm{GeV}$ at generator level (using d0mess [65]) were processed using the full detector simulation and reconstructed with DØReco version p14.06.01. 
A weight factor $w\left(p_{T}^{\mu}\right)$ for each muon of the QCD Monte Carlo simulation is determined like

$$
w\left(p_{T}^{\mu}\right)=\frac{\mathrm{N}_{\text {isolated }}^{\mu}}{\mathrm{N}_{\text {anti-isolated }}^{\mu}}\left(p_{T}^{\mu}\right) \cdot \sqrt{\frac{\mathrm{N}_{\text {anti-isolated }}^{\mu}}{\mathrm{N}_{\text {isolated }}^{\mu}}}
$$

where $\mathrm{N}_{\text {isolated }}^{\mu}\left(\mathrm{N}_{\text {anti-isolated }}^{\mu}\right)$ is the total number of isolated (anti-isolated) muons from $b \bar{b}$ decays. The term with the square root in equation (4.4) (the square root) takes into account that the weight factors are calculated from a sample where both muons are either isolated or anti-isolated.

The samples of isolated and anti-isolated muons have different relative fractions of direct $b \rightarrow \mu$, cascade $b \rightarrow c \rightarrow \mu$ and charm $c \rightarrow \mu$ decays. Therefore $w\left(p_{T}^{\mu}\right)$ is calculated separately for events with opposite-sign and same-sign muon pairs. Figure 4.4 a),b) shows the linear fit-function for the weight factors of the two leading $p_{T}$ muons in opposite-sign muon events. With increasing transverse momentum, the total number of isolated muons
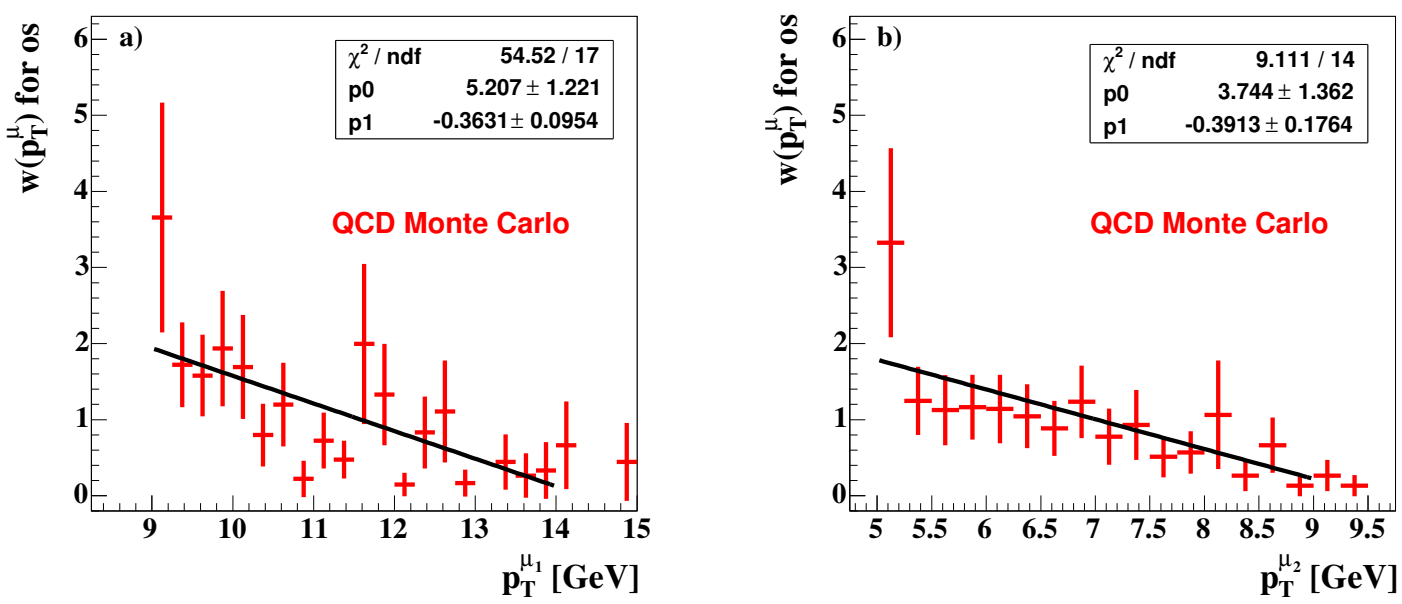

Figure 4.4: Weight factor $w\left(p_{T}^{\mu}\right)$ for $\mu_{1}$ and $\mu_{2}$ in events with opposite-sign (os) muons.

decreases, leading to a small weight $w\left(p_{T}^{\mu}\right)$ for these muons. The two leading $p_{T}$ muons in same-sign muon events have the same linear fit-functions within errors, shown in figure 4.5 a),b) respectively. Same-sign muon events are produced when one b quark decays directly and the $\bar{b}^{\prime}$ quark decays via a cascade. The transverse momentum of these muons is small, especially $p_{T}^{\mu_{2}}$ for the second muon.

For muons with higher transverse momentum, the QCD Monte Carlo ${ }^{5}$ statistics is not sufficient to determine the weight factors. In order to describe the $p_{T}$ distributions of these muons correctly, the weight factor is kept at a minimum of 0.4 . The linear extrapolation of the fit-functions described above, is less than 0.4 for muons with $p_{T}^{\mu_{1}}>13 \mathrm{GeV}\left(p_{T}^{\mu_{2}}>\right.$ $8 \mathrm{GeV}$ ) for the leading (second leading $p_{T}$ muon).

\footnotetext{
5 To produce the QCD Monte Carlo sample, 10 Million $b \bar{b}$ events were generated. From these events only a fraction of $0.03 \%$ passed the preselection from which a fraction of $12 \%$ passed the selection cuts for the transverse momenta and isolation.
} 

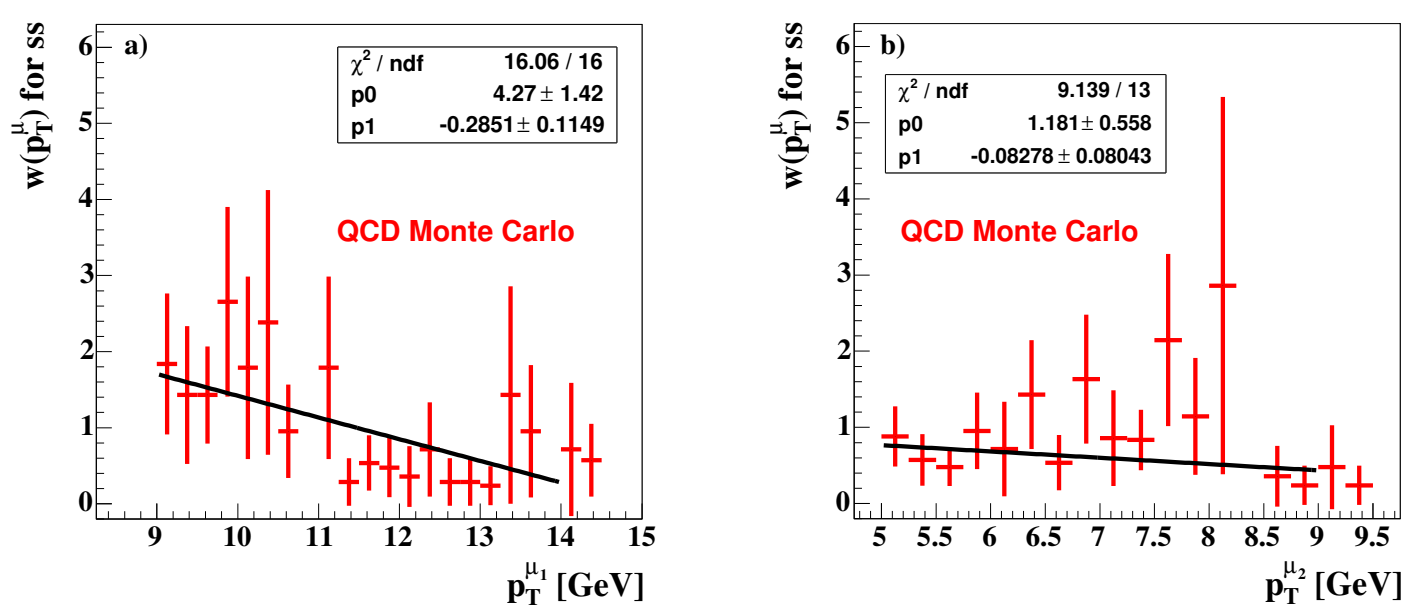

Figure 4.5: Weight factor $w\left(p_{T}^{\mu}\right)$ for $\mu_{1}$ and $\mu_{2}$ in events with same-sign (ss) muons.

In a second step each event from the data sample of anti-isolated muons was weighted with $w\left(p_{T}^{\mu_{1}}\right) \cdot w\left(p_{T}^{\mu_{2}}\right)$ corresponding to the transverse momenta of the muons. In order to include correlation effects between the two muons an overall normalization factor is calculated by choosing a region where the signal contribution is low. SUSY signal events are expected to have a large amount of missing transverse energy $\left(\mathbb{E}_{T}\right)$ in the event, whereas it is small for QCD events. Requiring $\mathbb{E}_{T}<15 \mathrm{GeV}$ at an early stage of the selection the signal contribution (or bias) is very low and negligible.

The distribution of thedimuon mass $M_{\mu \mu}$ for events with isolated opposite-sign (samesign) muons is shown in figure 4.6 a),b) in the kinematic region described before. The data events are shown as dots with error bars and the green filled histogram shows the events expected from Drell-Yan processes. Subtracting the events from Drell-Yan processes from the data events $\left(\mathrm{N}_{\mathrm{isol}}^{\mathrm{DATA}}-\mathrm{N}_{\mathrm{isol}}^{\mathrm{Z} / \gamma^{*}}\right)$, the QCD events with isolated muons are left as a function of $M_{\mu \mu}$ (the yellow filled histogram). In the case of the events with isolated same-sign muons, the Drell-Yan background is as small as shown in figure $4.6 \mathrm{~b}$ ). The $\mathbb{E}_{T}$ in all events shown is less than $15 \mathrm{GeV}$.

The QCD events with isolated muons must be compared with the estimated QCD background from data events with anti-isolated muons, where each event was weighted $\left(w\left(p_{T}^{\mu_{1}}\right) w\left(p_{T}^{\mu_{2}}\right) \mathrm{N}_{\text {anti-isol }}^{\text {DATA }}\right)$ The weights were obtained as described in equation (4.4). The estimated QCD background is then normalized with a factor of $s^{o s}=0.83$ and $s^{s s}=0.89$ for events with opposite-sign and same-sign muons, respectively. This is shown in figure 4.7. The yellow histogram is like in figure 4.6 and the open red histogram shows the weighted data events with anti-isolated muons. It describes the QCD events with isolated muons correctly.

In summary, the QCD events $\mathrm{N}_{\text {isolated }}^{\mathrm{QCD}}$ can be estimated from a data sample with antiisolated muon events. This is confirmed by both distributions in figure 4.6. The weight 

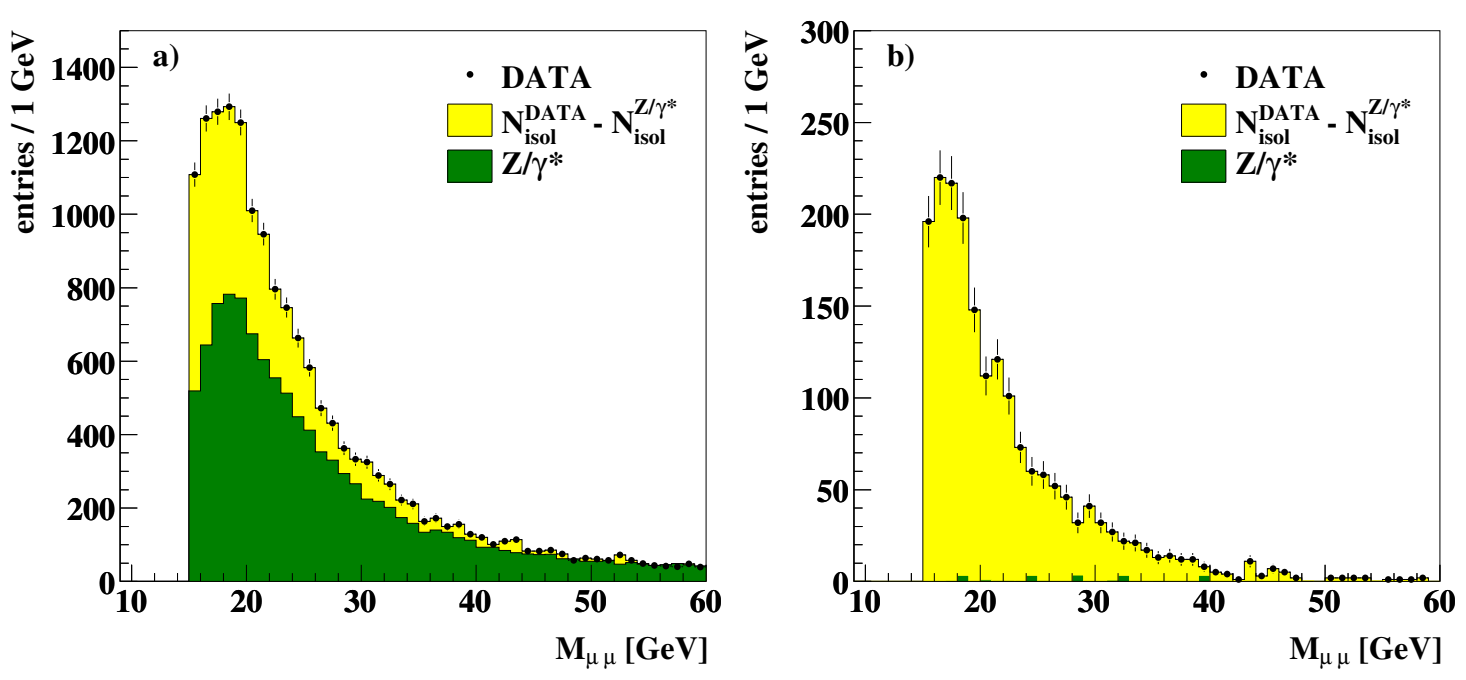

Figure 4.6: Distribution of $M_{\mu \mu}$ for events with isolated opposite-sign (same-sign) muons in the kinematic region $\mathbb{E}_{T}<15 \mathrm{GeV}$. Dots are data, the green filled histogram shows the expected Drell-Yan background $\left(Z / \gamma^{*} \rightarrow \mu \mu(\tau \tau)\right)$. The yellow histogram shows the QCD events with isolated muons.
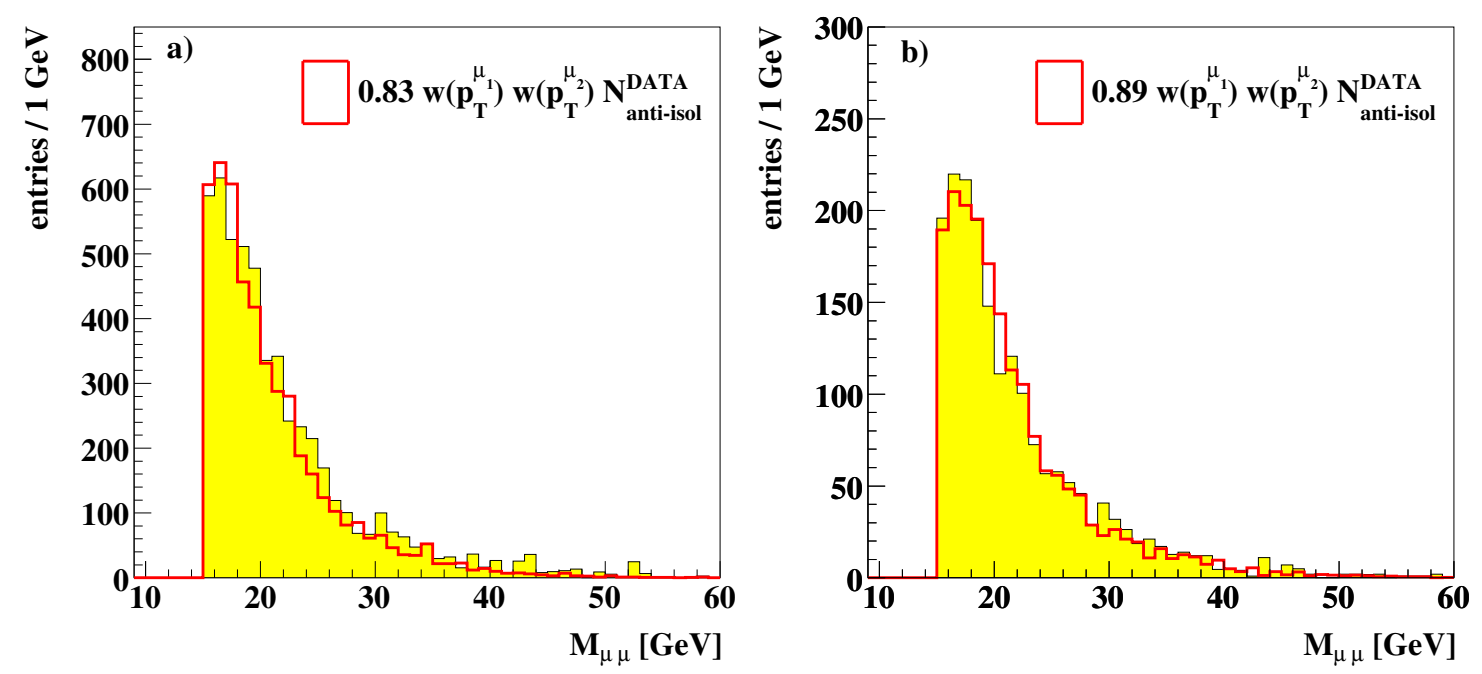

Figure 4.7: The $M_{\mu \mu}$ distribution for the QCD events with isolated opposite-sign and same-sign muons (yellow histogram a) and b) ) for $\mathbb{E}_{T}<15 \mathrm{GeV}$. The red line shows the distribution for weighted data events with anti-isolated muons. 
factor $w\left(\mu_{1}, \mu_{2}\right)$ from equation (4.3) is then defined as

$$
w\left(\mu_{1}, \mu_{2}\right)=s^{o s(s s)} \cdot w\left(p_{T}^{\mu_{1}}\right) \cdot w\left(p_{T}^{\mu_{2}}\right)
$$

for events with opposite-sign or same-sign muons. The weight factor $w\left(\mu_{1}, \mu_{2}\right)$ was obtained in a QCD dominated region with a small amount of $\mathbb{E}_{T}$. In the following it is tested if the data can be described correctly, if $\mathbb{E}_{T}$ is not restricted.

Figure 4.8 shows the $M_{\mu \mu}$ distribution for events with opposite-sign and same-sign muon pairs after the preselection with no restiction for $\mathbb{E}_{T}$. The muons are isolated as
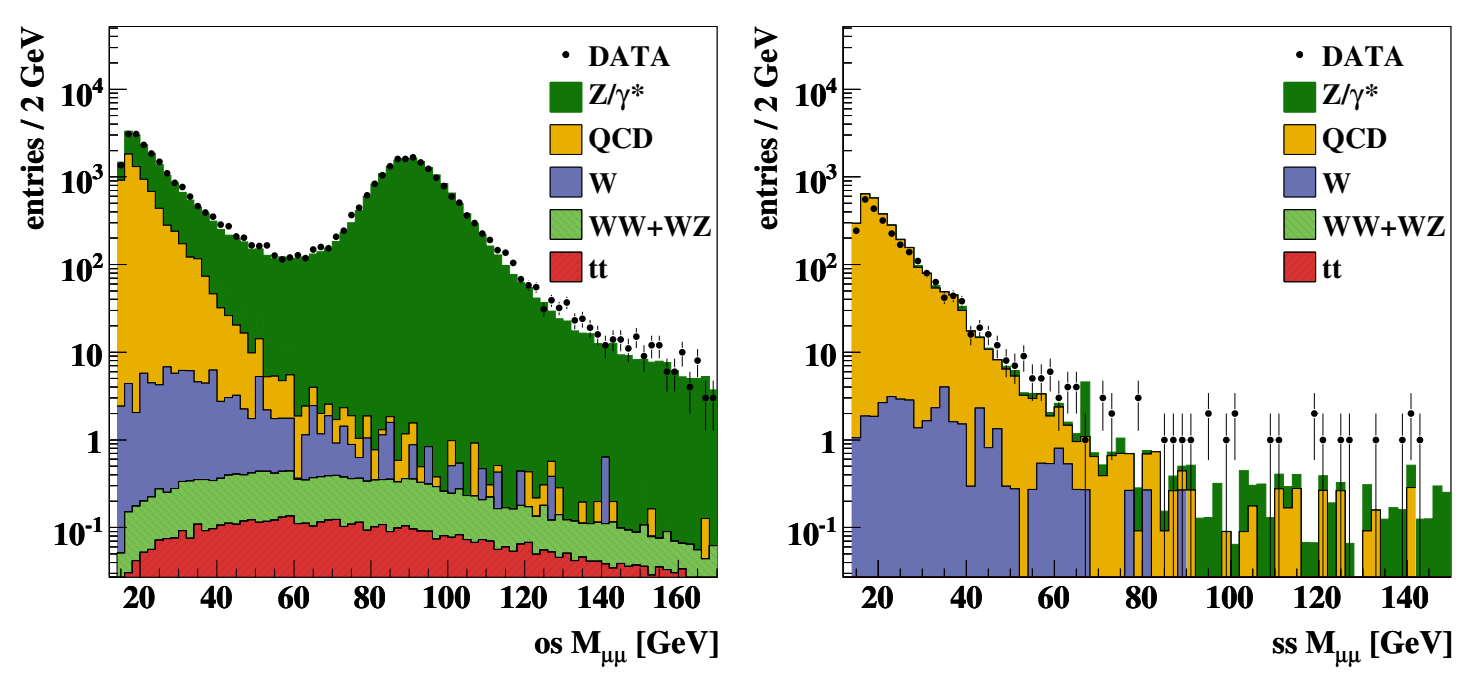

Figure 4.8: The reconstructed invariant dimuon mass $M_{\mu \mu}$ of events with opposite-sign and same-sign muons after preselection. The various Standard Model backgrounds are summed up including the QCD background.

described in section 4.5.1 for all Standard Model backgrounds. The QCD background is determined as described by equation (4.3). The data events are described correctly only if the QCD background is added to the Standard Model backgrounds of Drell-Yan, W, WW, $\mathrm{WZ}$ and $t \bar{t}$. In the case of same-sign muons the selected data events are primarily QCD events.

The elaborated method describes the data very well. The validity of the method was also tested by varying the linear-fit results and various selection variables. The results obtained were used to evaluate the systematic uncertainty for the modeling of this background (see section 5.5).

In the following, QCD background always means events determined as described above. All constant values in equation (4.5) are kept throughout the whole analysis. 


\section{Chapter 5}

\section{Trilepton Analysis}

The search for Supersymmetry events in the trilepton decay channel with two muons $q \bar{q} \rightarrow \widetilde{\chi}_{1}^{ \pm} \widetilde{\chi}_{2}^{0} \rightarrow \ell^{ \pm} \vee \widetilde{\chi}_{1}^{0} \mu^{ \pm} \mu^{\mp} \widetilde{\chi}_{1}^{0}$ depends on the efficient rejection of the Standard Model backgrounds, while retaining the Supersymmetry signal (SUSY signal). A dedicated event selection has been tuned on Monte Carlo and then applied to the data.

\subsection{Motivation of Selection Cuts}

Several points in the mSUGRA parameter space were examined to probe the SUSY signal $^{1}$. The Feynman diagram in figure 5.1 shows how associated charginos and neutralinos $\left(\widetilde{\chi}_{1}^{ \pm} \widetilde{\chi}_{2}^{0}\right)$ can be produced at Fermilab. For example, an u quark and a d antiquark, initially

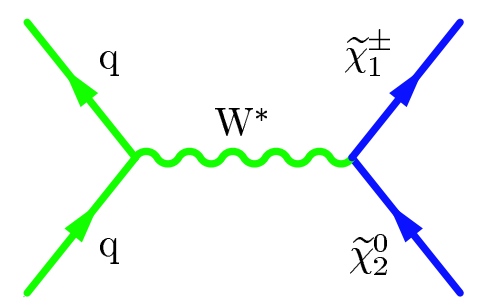

Figure 5.1: The $q \bar{q}$ annihilation into virtual $W^{*}$ and the production of a $\widetilde{\chi}_{1}^{ \pm} \widetilde{\chi}_{2}^{0}$ pair.

in the proton and anti-proton, annihilate into a virtual charged $\mathrm{W}$ boson $\left(W^{*}\right)$, producing a $\widetilde{\chi}_{1}^{ \pm} \widetilde{\chi}_{2}^{0}$ pair. The subsequent decays into leptons and LSPs via virtual boson or slepton exchange, defines the characteristics of the final state.

Figure 5.2 shows the Feynman diagram of the $\widetilde{\chi}_{1}^{ \pm} \widetilde{\chi}_{2}^{0}$ decay via virtual sleptons as an example for the investigated decay channel. The final state is characterized by two muons $\mu^{+} \mu^{-}$, an additional charged lepton $\left(\mu^{ \pm}, e^{ \pm}, \tau^{ \pm}\right)$and a large amount of missing transverse energy due to the $\widetilde{\chi}_{1}^{0}$ and $v$ which escape detection. This is a clean signature with isolated leptons.

\footnotetext{
${ }^{1}$ The theoretical background is given in section 2.5.4 and the Monte Carlo simulation of the SUSY signal is described in section 4.2.1.
} 


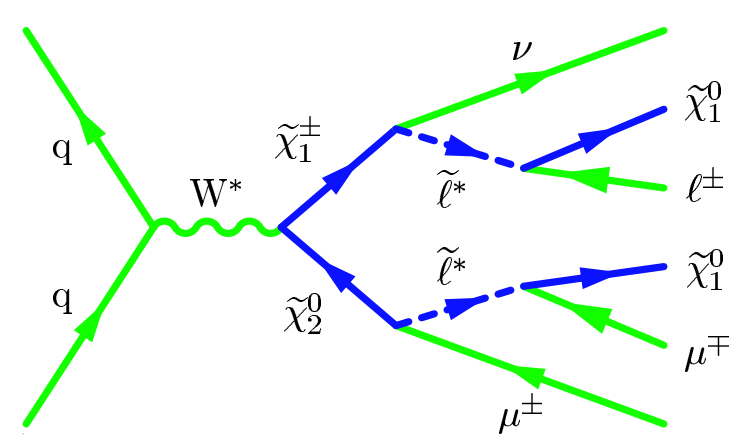

Figure 5.2: The $q \bar{q} \rightarrow \widetilde{\chi}_{1}^{ \pm} \widetilde{\chi}_{2}^{0} \rightarrow 3 \ell+2 \widetilde{\chi}_{1}^{0}+v$ decay via virtual sleptons.

The SUSY signal has some distinct kinematic characteristics. To determine the selection variables, generator level Monte Carlo was used, which means, that neither detector acceptance nor reconstruction code is applied on the Monte Carlo simulation. The generator level Monte Carlo has the advantage that each particle has an associated ID number ([5]) that identifies the particle species. Thus, the properties of the $\widetilde{\chi}_{1}^{ \pm} \widetilde{\chi}_{2}^{0}$ can be studied with confidence. This distinctive identification is only possible at generator level.

\section{Missing transverse energy $\left(\not_{T}\right)$}

Events with supersymmetric particles have a large amount of missing energy, because the $\widetilde{\chi}_{1}^{ \pm} \widetilde{\chi}_{2}^{0}$ decay into final states with two $\widetilde{\chi}_{1}^{0}$. They can be observed in the unbalanced transverse momentum of the event. Figure 5.3 shows the $\mathbb{E}_{T}$ distribution for a SUSY

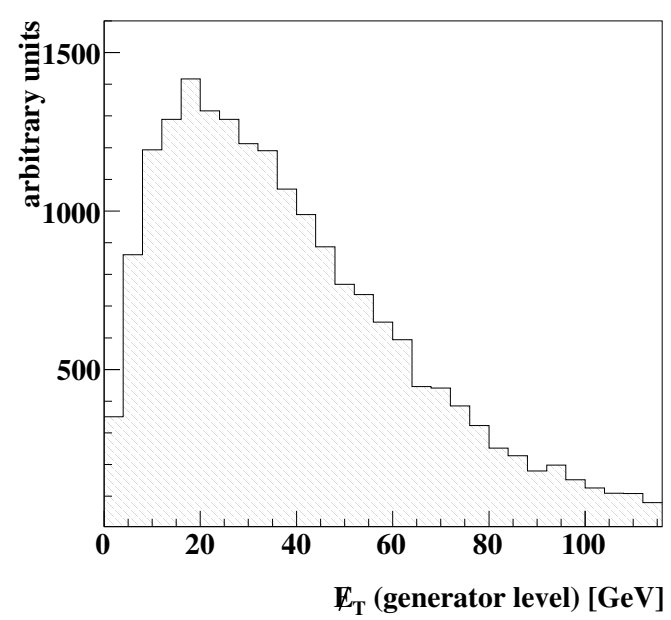

Figure 5.3: At generator level: the $\mathbb{E}_{T}$ distribution.

signal event. The fraction of energy carried away by the two $\widetilde{\chi}_{1}^{0}$ is quite large and depends on the mSUGRA parameters. The distribution shown for SUSY point D3 is similar for all SUSY points. In the actual event selection, the amount of $\mathbb{E}_{T}$ in the event discriminates 
the SUSY signal from the Standard Model backgrounds, which typically have a lower amount of $\mathscr{E}_{T}$ in their final state.

\section{Reconstructed invariant mass}

The invariant mass of the opposite-sign muons from the subsequent $\widetilde{\chi}_{2}^{0}$ decay, is shown in figure $5.4 \mathrm{a}$ ). The two muons are chosen by their ID numbers and it has been ensured, that they both arise from a $\widetilde{\chi}_{2}^{0}$ decay. The muons combine at a considerably low mass. The sharp edge for high masses $\left(M_{\mu^{ \pm} \mu^{\mp}} \sim 52 \mathrm{GeV}\right)$ is bound by the mass difference $m_{\widetilde{\chi}_{2}^{0}}-m_{\widetilde{\chi}_{1}^{0}}$ of the two lightest neutralinos, which corresponds to SUSY point D3.
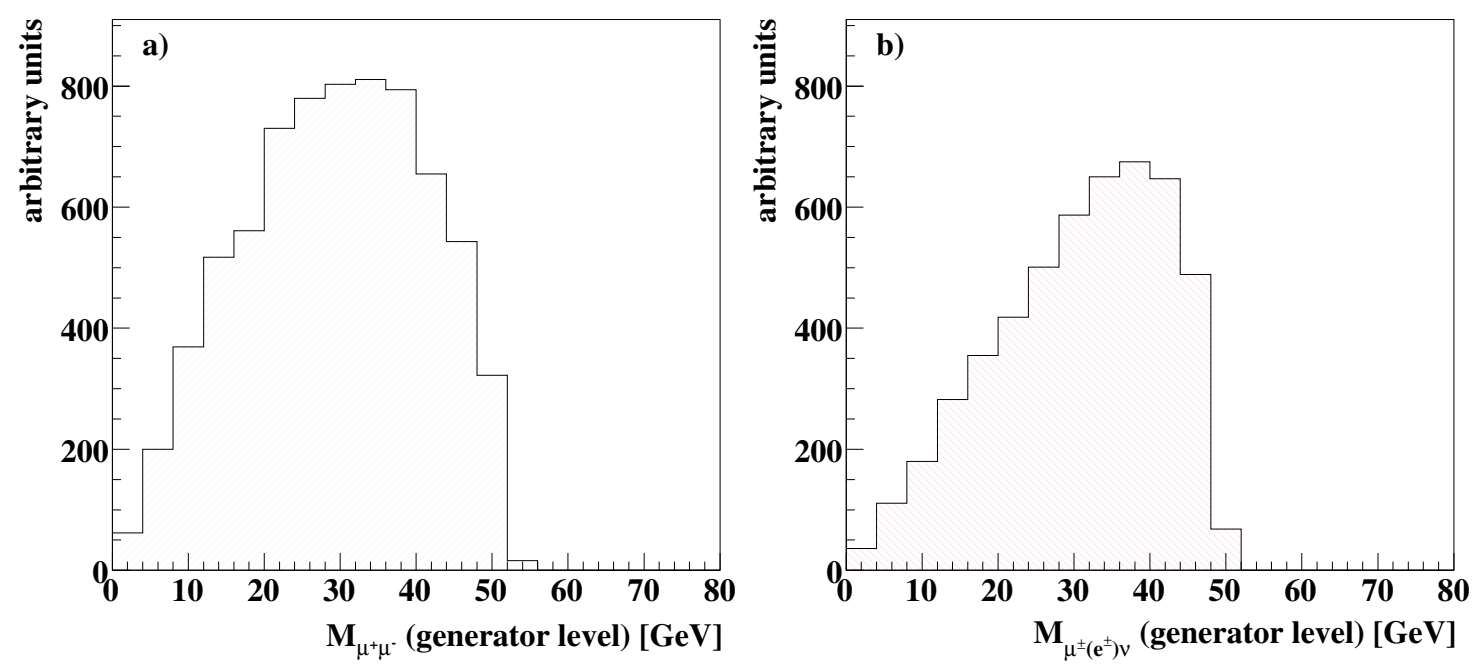

Figure 5.4: At generator level: the reconstructed invariant mass $M_{\mu^{ \pm} \mu^{\mp}}$ of the muons from the $\widetilde{\chi}_{2}^{0}$ decay and of the neutrino and the electron (muon) from the $\widetilde{\chi}_{1}^{ \pm}$decay.

The invariant mass of the additional charged lepton and the neutrino in the event is shown in figure $5.4 \mathrm{~b})$. The sharp edge for high masses $\left(M_{\mu^{ \pm}\left(e^{ \pm}\right) v} \sim 48 \mathrm{GeV}\right)$ corresponds to the mass difference $m_{\widetilde{\chi}_{1}^{ \pm}}-m_{\widetilde{\chi}_{1}^{0}}$ of the chargino and the LSP of SUSY point D3. Both leptons arise from the subsequent decay of the $\widetilde{\chi}_{1}^{ \pm}$.

In the actual event selection, a combination of two opposite sign muons must not necessarily combine the muons from the $\widetilde{\chi}_{2}^{0}$ decay. A combination of two muons where one is from the chargino and the other from the neutralino decay is possible. An invariant mass can also be reconstructed from same-sign muons when selecting one muon from $\tilde{\chi}_{1}^{ \pm}$and the other from the $\widetilde{\chi}_{2}^{0}$ decay. For this reason, an upper invariant mass cut for any pair of the selected two muons will be applied in the actual event selection. The muon pairs can be of opposite or same sign.

The requirement of a third charged lepton $\left(\ell^{ \pm}\right.$in figure 5.2) is replaced with the requirement of an isolated track in the later analysis. The invariant mass of the track (of any sign) and a selected muon, will also be analyzed. 


\section{Scenarios with soft leptons}

The presented analysis is sensitive in a mSUGRA parameter space for low sparticle masses. This means masses of $\sim 100 \mathrm{GeV}$ for the gauginos. The transverse momentum $p_{T}$ of the muons and the leptons in the final state depends also on the mass difference between the gauginos and the LSP: $m_{\widetilde{\chi}_{1}^{ \pm}\left(\widetilde{\chi}_{2}^{0}\right)}-m_{\widetilde{\chi}_{1}^{0}}$. The following scenarios can be defined:

$m_{\tilde{\ell}}<m_{\tilde{\chi}_{1}^{ \pm}}$and $m_{\tilde{\ell}}<m_{\widetilde{\chi}_{2}^{0}}$ :

if sleptons are light enough $\widetilde{\chi}_{1}^{ \pm} \widetilde{\chi}_{2}^{0}$ decay via on-shell sleptons.

The mass difference $m_{\tilde{\ell}}-m_{\tilde{\chi}}$ is crucial for the selection efficiency. For small mass difference $\left|m_{\tilde{\chi}_{2}^{0}}-m_{\tilde{\ell}}\right|$ the $\tilde{\ell}$ is produced with a very soft lepton, which reduces the selection efficiency.

$m_{\tilde{\ell}} \geq m_{\widetilde{\chi}_{2}^{0}}:$

the leptonic branching fraction is maximally enhanced by the three-body decays via virtual sleptons $\tilde{\ell}^{*}$. The decay of the neutralino via real stau into taus is suppressed (as staus are in the two-body decay region). The leptons have a large $p_{T}$ and can be detected.

\section{large- $m_{\tilde{\ell}}:$}

for large slepton masses $m_{\tilde{\ell}}$ the $\widetilde{\chi}_{1}^{ \pm} \widetilde{\chi}_{2}^{0}$ decay via virtual gauge bosons. The leptonic branching fraction is about $3 \%$.

The leptonic branching fraction of the chargino and neutralino depends on the relative contribution from slepton and gauge bosons exchange graphs (see section 2.5.4). It varies as a function of the slepton masses.

\section{Muon transverse momentum}

The transverse momenta of the muons coming from the subsequent decays of the $\widetilde{\chi}_{2}^{0}$ and $\widetilde{\chi}_{1}^{0}$ can get very small. Three typical SUSY points are chosen to illustrate the scenarios discussed above. The neutralino mass is $m_{\widetilde{\chi}_{2}^{0}}=114 \mathrm{GeV}$ for the three scenarios presented.

Figure 5.5 a) shows the transverse momentum distribution at generator level for SUSY point D2. Neutralinos can decay via real sleptons. When the neutralino masses are equal or smaller than the slepton masses, $\widetilde{\chi}_{1}^{ \pm} \widetilde{\chi}_{2}^{0}$ decay via virtual sleptons. Figure $5.5 \mathrm{~b}$ ) shows the transverse momentum distribution at generator level for SUSY point D3. In the scenario with large slepton masses, the decay via virtual gauge bosons becomes dominant. Figure $5.5 \mathrm{c}$ ) shows the transverse momentum distribution at generator level for SUSY point D6.

For the actual event selection, presented in the next section, an asymmetric cut is applied for the two leading $p_{T}$ muons. Additionally, the requirement to find a third charged lepton $\left(\mu^{ \pm}, e^{ \pm}, \tau^{ \pm}\right)$in the event is replaced with the requirement to find an isolated track in the event. 

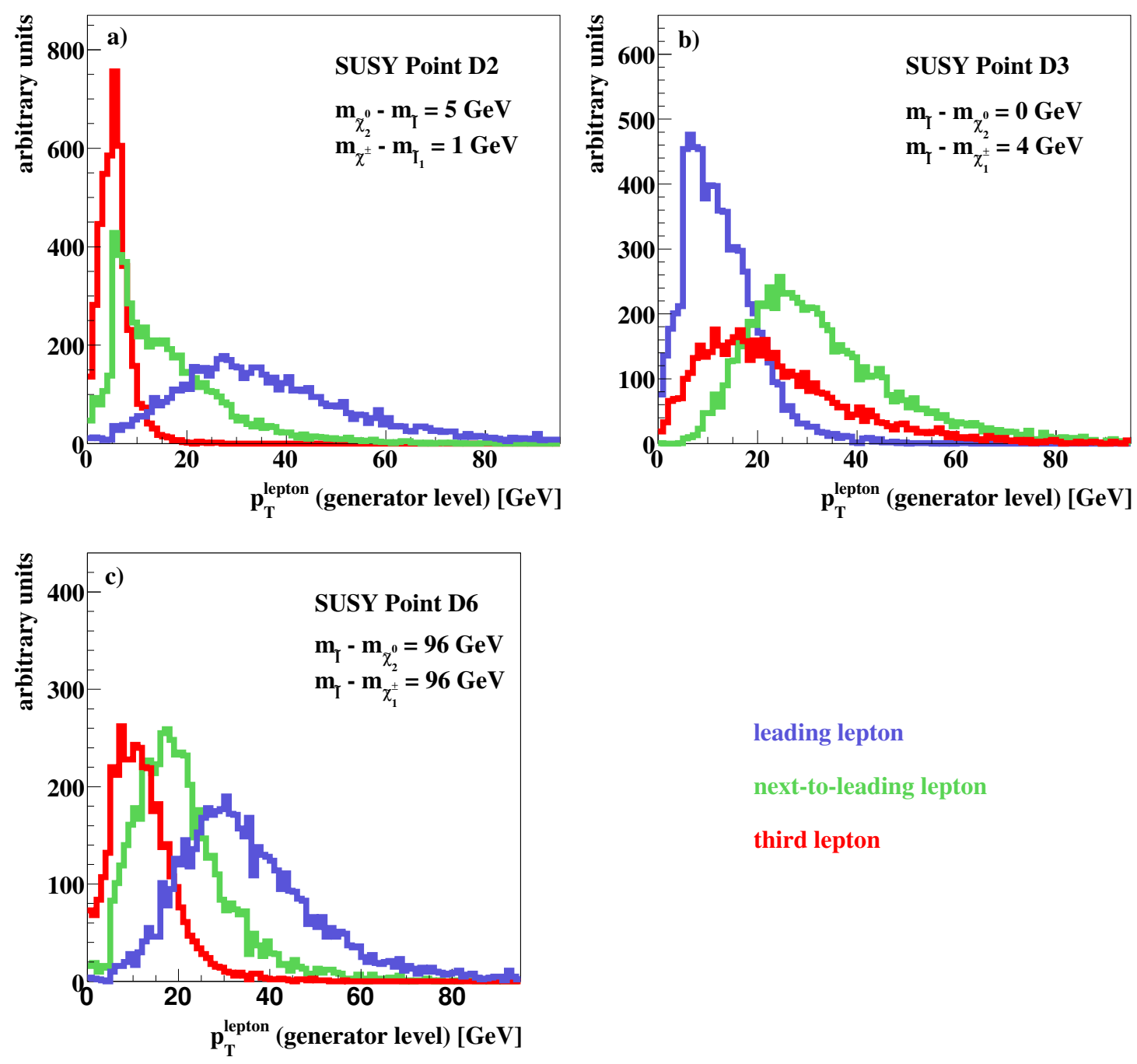

leading lepton

next-to-leading lepton

third lepton

Figure 5.5: At generator level: the transverse momentum distribution in the $\mu^{+} \mu^{-} \mu^{ \pm}\left(e^{ \pm}\right)$ final state for the leading lepton (blue line), the next-to-leading lepton (green line) and the third lepton (red line). 


\subsection{Event Selection}

The following event selection is based on the kinematic properties of the particles in the final state. As seen in the previous section the SUSY signal under investigation provides some distinct characteristics. These allow to find a set of variables where cuts are successively applied on.

In the following several distributions are presented, which describe the kinematic properties of the physics processes involved and motivate the considered best combination of variables and applied cuts. This rejects events similar to those from the Standard Model backgrounds, while efficiently retaining the SUSY signal. The cuts are applied simultaneously on Monte Carlo and data events.

\section{Preselection}

The $\widetilde{\chi}_{1}^{ \pm} \widetilde{\chi}_{2}^{0} \rightarrow \mu \mu \ell+\mathscr{E}_{T}$ decay channel requires at least two muons in the final state. Therefore the main Standard Model backgrounds for the SUSY signal can be briefly classified into three categories, according to their final state. The muons can originate from direct decays and also from jets in the event:

\begin{tabular}{cc} 
final state & Standard Model background \\
\hline two muons + additional lepton & $W Z \rightarrow \mu^{+} \vee \mu^{-} \mu^{+}, W Z \rightarrow e^{+} \vee \mu^{-} \mu^{+}$ \\
up to two muons & $Z / \gamma^{*} \rightarrow \mu^{+} \mu^{-}, Z / \gamma^{*} \rightarrow \tau^{+} \tau^{-}$ \\
$W W \rightarrow \mu^{+} \vee \mu^{-} \bar{v}, t \bar{t} \rightarrow \mu^{ \pm} \vee \mu^{ \pm} \vee b \bar{b}$ \\
$W \rightarrow \mu^{ \pm} v, \mathrm{QCD}$
\end{tabular}

Table 5.1: The Standard Model backgrounds classified according to their final state.

A loose preselection has been performed to selected events with two loose muons with $p_{T}^{\mu_{1}}>9 \mathrm{GeV}$ and $p_{T}^{\mu_{2}}>5 \mathrm{GeV}$ from the data and Monte Carlo samples. The muons in the final state of the SUSY signal are isolated. The isolation criteria are described in section 4.5.1. The $p_{T}$ distribution of the two leading- $p_{T}$ muons $\left(p_{T}^{\mu_{1}}\right.$ and $p_{T}^{\mu_{2}}$ ) are shown in figure 5.6 a), b), respectively. The Standard Model background events are summed up and compared with the data events. The discrepancy between the data and the sum of backgrounds for high- $p_{T}$ muons is due to the momentum resolution in data and Monte Carlo simulations. The tails in the resolutions are underestimated in the Monte Carlo simulations. These events are mostly removed with the cut on the reconstructed invariant mass from the two muons. The distribution of the SUSY signal, also for all following distributions, is shown as an open histogram (black line). The SUSY point in all distributions corresponds to D3 in table 4.1.

The dominating background after the preselection is the Drell-Yan background $Z / \gamma^{*}$. It contains two well isolated muons in the final state originating from direct decays. The peak clearly visible for events with high- $p_{T}$ muons at $p_{T} \sim 40 \mathrm{GeV}$ in both distributions, belongs to muons which reconstruct the $Z$ boson mass. Background events from QCD production have mostly low- $p_{T}$ muons in their final states. The isolation requirement 

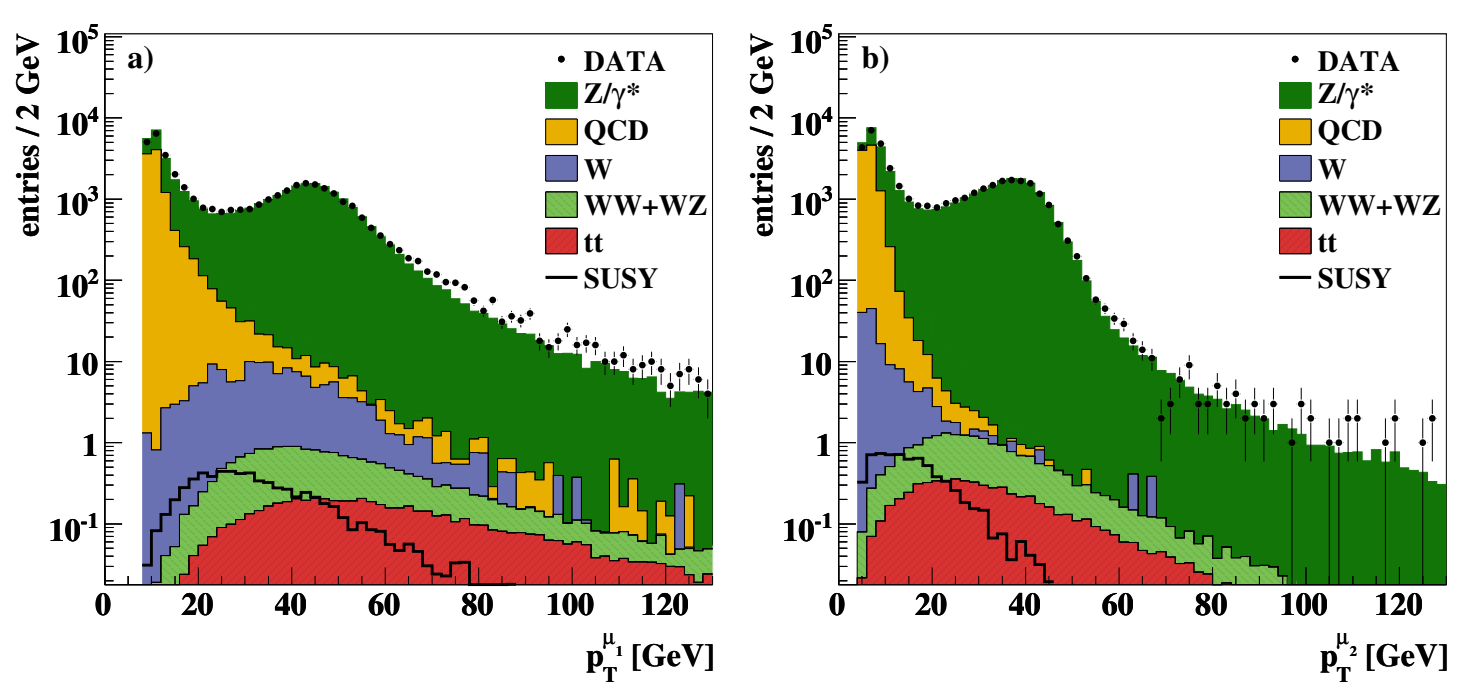

Figure 5.6: The transverse momentum for the first muon $p_{T}^{\mu_{1}}$ and second muon $p_{T}^{\mu_{2}}$ at preselection. The Standard Model backgrounds are: Drell-Yan $\left(Z / \gamma^{*} \rightarrow \mu^{+} \mu^{-}, Z / \gamma^{*} \rightarrow\right.$ $\tau^{+} \tau^{-}$) (green filled histogram), QCD (yellow filled histogram), $W^{ \pm}$(blue filled histogram), $W W \rightarrow \mu^{+} \vee \mu^{-} \bar{v}, W Z \rightarrow \mu^{+} \vee \mu^{-} \mu^{+}$and $W Z \rightarrow e^{+} v \mu^{-} \mu^{+}$(green hashed histogram), $t \bar{t} \rightarrow \mu^{ \pm} \vee \mu^{ \pm} v b \bar{b}$ (red hashed histogram). The SUSY signal (Point D3) is shown as a black line (open histogram).

already reduced this background significantly. The SUSY signal also requires low- $p_{T}$ muons in the final state. The contribution from $\mathrm{W}, \mathrm{WW}$ and $t \bar{t}$ backgrounds are small compared to the main backgrounds. Especially the irreducible background WZ is small at this early stage of selection. The sum of the Standard Model background events agrees with the data events. It should be noticed, that especially the QCD background contribution, determined from a sample of data events with anti-isolated muons, is describing the data correctly.

The $p_{T}$-cuts are kept at a low value at the stage of preselection. This assures sufficient statistics for the estimation of the QCD background from data, discussed in section 4.6, where the distribution of $M_{\mu \mu}$ at preselection stage is also shown.

\subsubsection{Muon Transverse Momenta: $p_{T}^{\mu_{1}}, p_{T}^{\mu_{2}}$}

To retain the selection efficiency for the SUSY signal, the two selected muons must pass a soft acceptance cut on $p_{T}^{\mu_{1}}$ and $p_{T}^{\mu_{2}}$. The SUSY signal events have a small transverse momentum for the second selected muon. Therefore, the acceptance cuts for the two leading- $p_{T}$ muons are chosen as

$$
p_{T}^{\mu_{1}}>11 \mathrm{GeV}, p_{T}^{\mu_{2}}>5 \mathrm{GeV} .
$$

Increasing the cut for the first muon from $9 \mathrm{GeV}$ to $p_{T}^{\mu_{1}}>11 \mathrm{GeV}$ significantly reduces the QCD background by $60 \%$. 


\subsubsection{Invariant Dimuon Mass: $M_{\mu \mu}$}

The reconstructed invariant dimuon mass of the two selected muons $\mu_{1}$ and $\mu_{2}$ is calculated according to

$$
M_{\mu \mu}=\sqrt{\left(E_{\mu_{1}}+E_{\mu_{2}}\right)^{2}-\left(\left(p_{x}^{\mu_{1}}+p_{x}^{\mu_{2}}\right)^{2}+\left(p_{y}^{\mu_{1}}+p_{y}^{\mu_{2}}\right)^{2}+\left(p_{z}^{\mu_{1}}+p_{z}^{\mu_{2}}\right)^{2}\right)}
$$

The selected muons can have charges of same sign or opposite sign. The invariant dimuon mass from opposite-sign and same-sign muons after the selection cut on the transverse momentum is shown in figure 5.7. The sum of backgrounds agrees with the data. The $\mathrm{Z}$

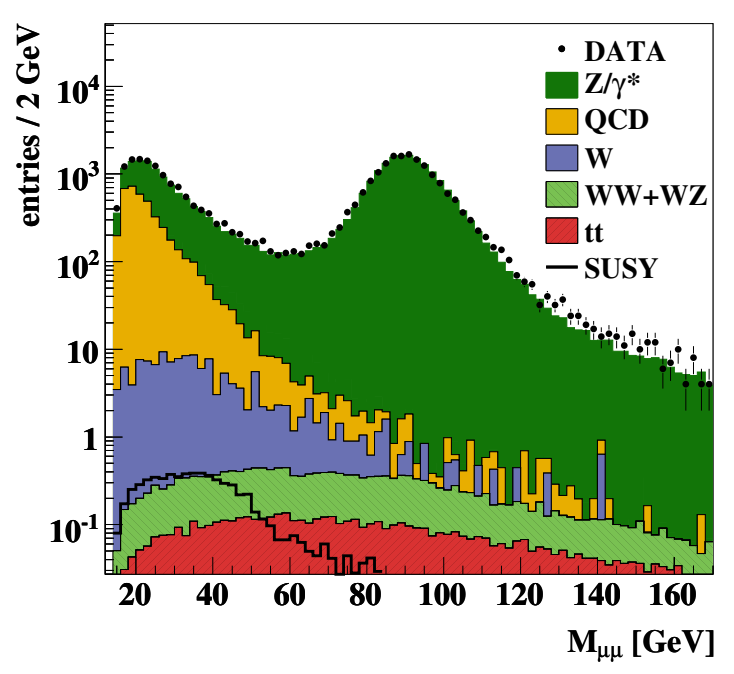

Figure 5.7: The invariant dimuon mass.

resonance from $Z \rightarrow \mu^{+} \mu^{-}$events is not in the SUSY signal region and those events can be rejected. The distribution for the corresponding invariant dimuon mass for opposite-sign and same-sign muons is shown separately in figure 5.8 a) and b), respectively.

The main backgrounds as well as the WZ background are shown separately in figure 5.9. The $Z / \gamma^{*}$ and $\mathrm{WZ}$ backgrounds show the expected $Z$ resonance from decays into muons (fig. 5.9 a), c)). Additionally, the $Z / \gamma^{*}$ and QCD backgrounds show a large contribution in the mass range $M_{\mu \mu}<20 \mathrm{GeV}$. The muons of the $Z / \gamma^{*}$ events below a mass of $M_{\mu \mu}<50 \mathrm{GeV}$ originate from $Z / \gamma^{*} \rightarrow \mu^{+} \mu^{-}$and $Z \rightarrow \tau^{+} \tau^{-}$decays. Also the QCD background has a large contribution in this mass range. Especially for events with samesign muons (see fig. $5.8 \mathrm{~b}$ )) this is the dominating background. The WZ background is negligibly small.

The SUSY signal distribution (see fig. 5.9 d)), has its main contribution in the mass range from $20 \mathrm{GeV}<M_{\mu \mu}<50 \mathrm{GeV}$, which is due to the mass difference between the neutralinos or charginos and the LSP. An event is accepted if

$$
15 \mathrm{GeV}<M_{\mu \mu}<50 \mathrm{GeV} .
$$

This cut on the invariant dimuon mass significantly reduces the $Z / \gamma^{*}$ background by $70 \%$. 

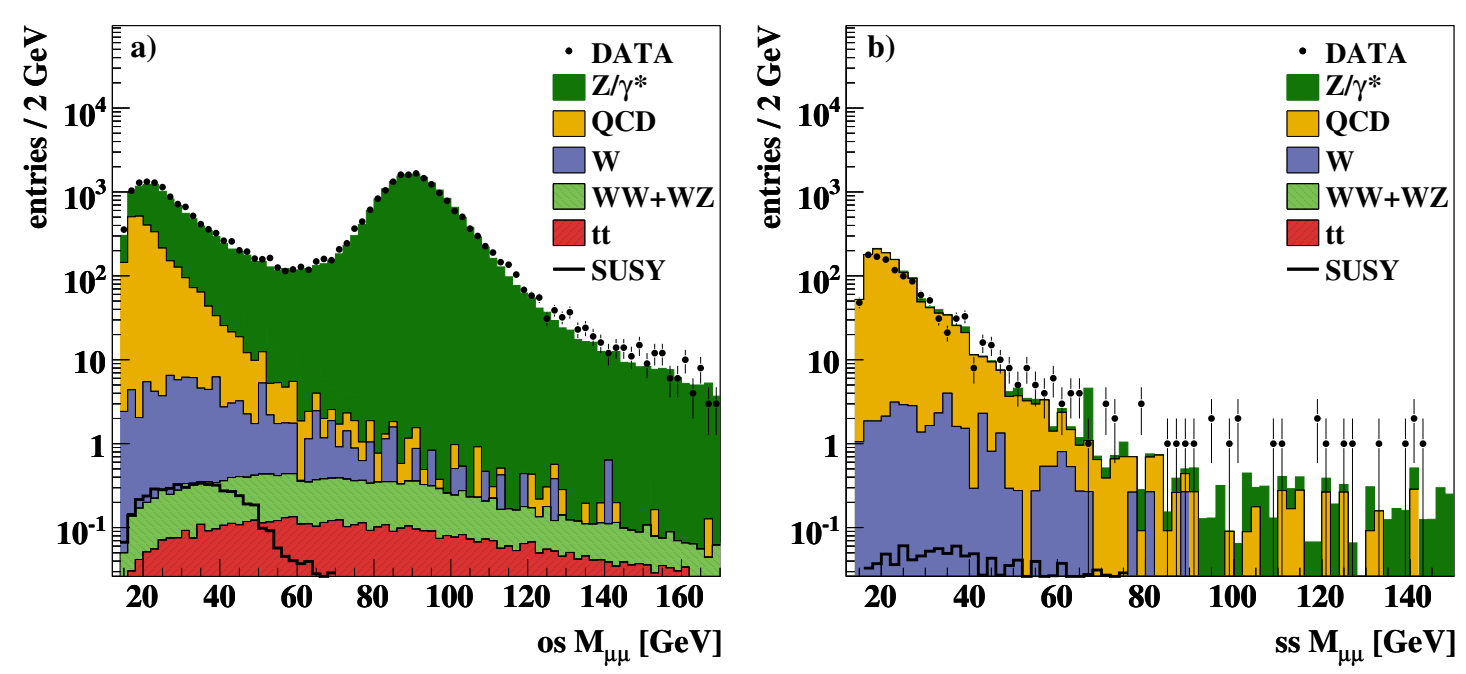

Figure 5.8: The invariant dimuon mass from opposite-sign (os) and same-sign (ss) muons.
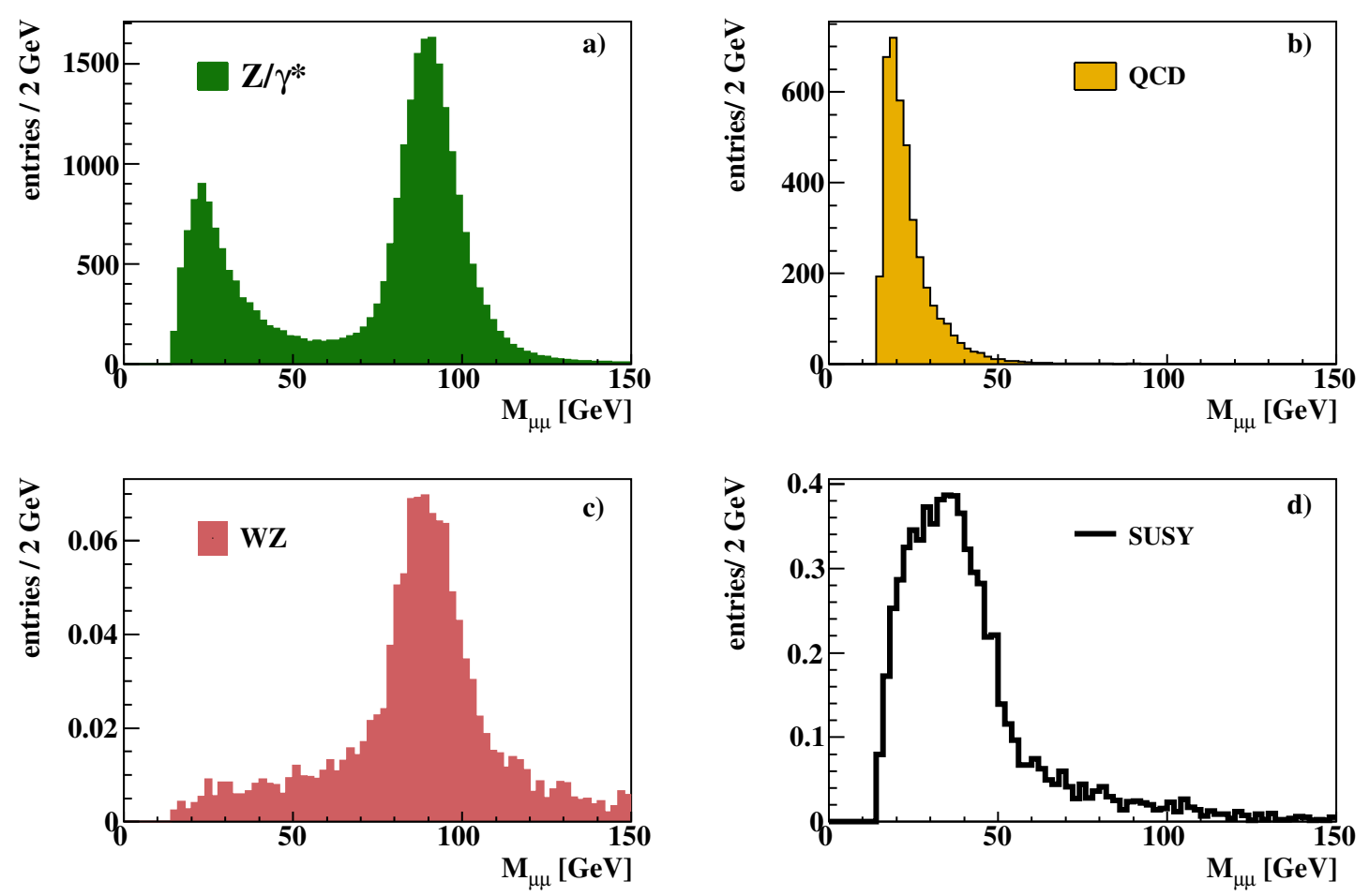

Figure 5.9: The invariant dimuon mass: $Z / \gamma^{*}$ and $Q C D$ background (upper row), $W Z$ and SUSY signal (lower row). 


\subsubsection{Missing Transverse Energy: $\mathbb{H}_{T}$}

The amount of missing transverse energy $\mathbb{E}_{T}$ for the SUSY signal is large compared to the remaining Standard Model backgrounds. It is the sum of the two $\widetilde{\chi}_{1}^{0}$ and the neutrino in the final state, which escape detection. For the Standard Model backgrounds W, WW, $\mathrm{WZ}$ the $\mathscr{E}_{T}$ in the event originates from the neutrinos in the final state.

The main backgrounds at this selection stage are shown separately in figure 5.10. The
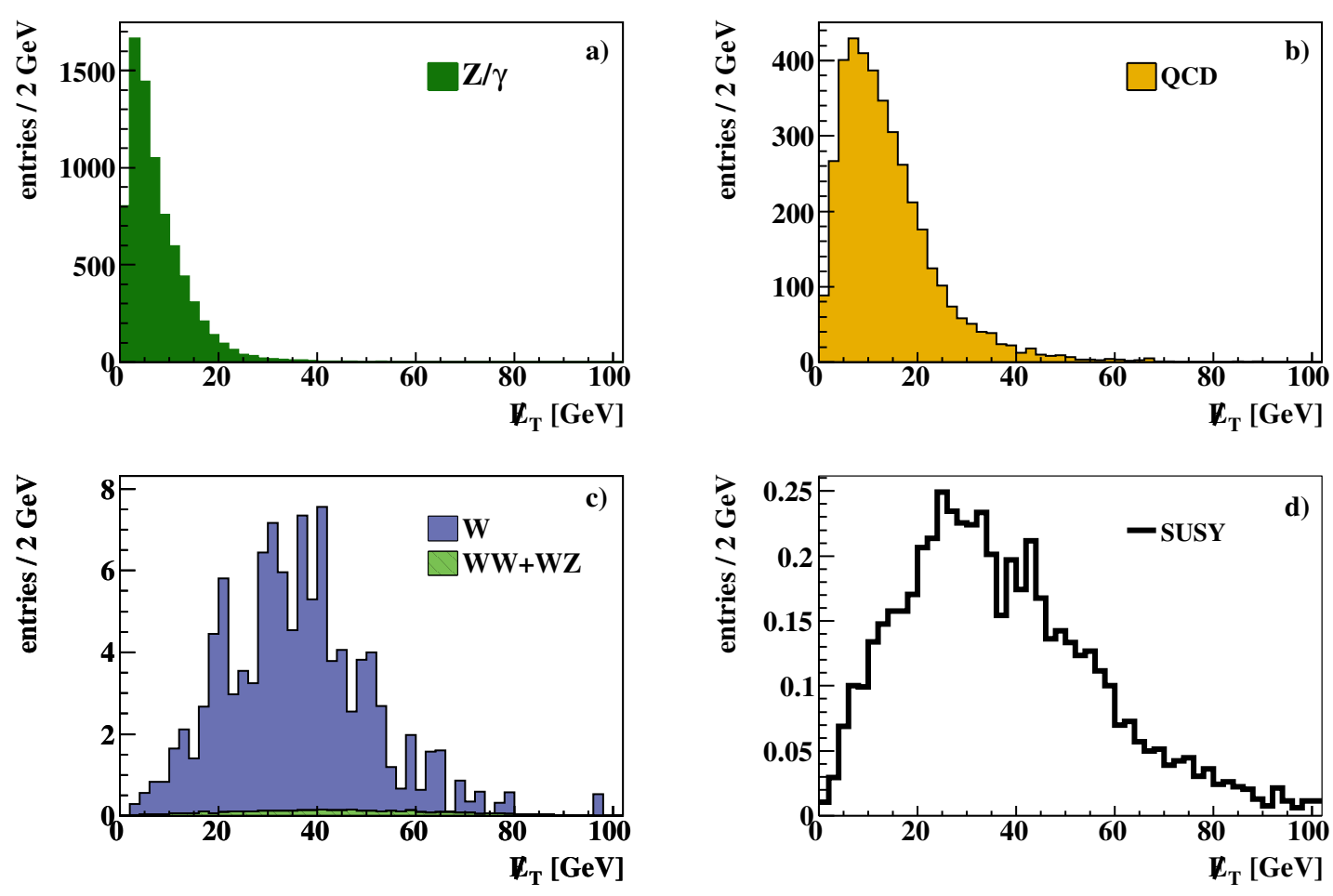

Figure 5.10: The missing transverse energy: $Z / \gamma^{*}$ and QCD (upper row), sum of W,WZ,WW (lower row left) and the SUSY signal (lower row right).

contribution from the Standard Model backgrounds $Z / \gamma^{*}$ and QCD (figure $5.10 \mathrm{a}$ ), b)) dominate the low $\mathbb{E}_{T}$ region, except for the $\mathrm{W}$ background, which shows larger fractions of $\mathbb{E}_{T}$ in the events (figure $5.10 \mathrm{c}$ )). The SUSY signal shows a large amount $\mathbb{E}_{T}$ as expected (figure $5.10 \mathrm{~d})$ ).

The $\mathbb{E}_{T}$ distribution for the sum of all Standard Model backgrounds is shown in figure 5.11. The $Z / \gamma^{*}$ background is still twice as big as the QCD background and is the dominant background source. Requiring an amount of missing transverse energy of

$$
\mathbb{E}_{T}>22 \mathrm{GeV}
$$

in the event, reduces both the Drell-Yan and the QCD background significantly. The W background is also reduced by $20 \%$. The $t \bar{t}$ background is also characterized by large $\mathbb{E}_{T}$. Its contribution is small and will be efficiently rejected with the following selection cuts. 


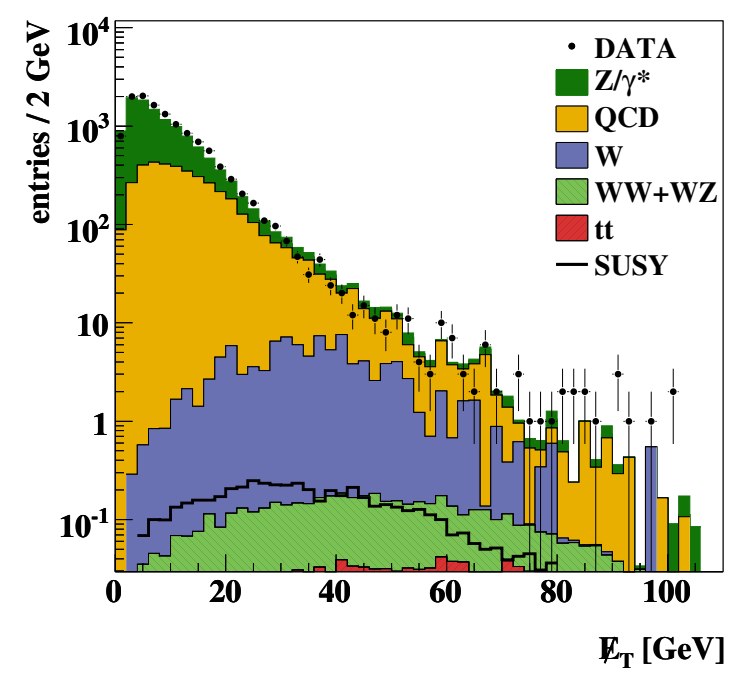

Figure 5.11: The missing transverse energy.

For the SUSY signal $80 \%$ of the events pass the cut, whereas only $10 \%$ of the sum of background sources survive this $\mathbb{E}_{T}$-cut. It should be mentioned, that this cut was optimized for several SUSY points, to get the highest efficiency possible in a parameter space, where the presented analysis is sensitive.

\subsubsection{Additional Isolated Track: $p_{T}^{t r}$}

The SUSY signal features a third charged lepton in the final state which yields a track in the detector. Requiring an identification of such a track as an electron, muon or tau, reduces the selection efficiency dramatically. Therefore instead of a charged lepton identification, only the track requirement is kept. The track must fulfill the following quality criteria:

- a transverse momentum of $p_{T}^{t r}$ larger than $3 \mathrm{GeV}$

- the $\chi^{2} / N d f$ of the track should be less than 3.0

- the track should be isolated with respect to all other tracks in a hollow cone of $\Sigma_{\text {track }}^{0.1<\Delta R<0.5} p_{T}<1 \mathrm{GeV}$

- the number of CFT hits should be greater than 13 or the total number of hits must exceed 17 and the number of SMT hits is greater than zero

The isolation criteria is designed to be efficient for electrons, muons and taus. All tau decays (leptonic, hadronic (1 prong), hadronic (3-prong)) either produce only one track or a set of tracks in a very narrow region in $\eta-\phi$. This is taken into account by the isolation of an hollow cone around the selected track. Additionally, the track must be 
well separated from the tracks of the other two muons with $\Delta R>0.5$ and must be from the same vertex as the selected muons within $1 \mathrm{~cm}$.

The $p_{T}^{t r}$ of the additional track is smeared with the same smearing function used for the smearing of the muon transverse momentum (equation (4.1)). The selected tracks are found in the pseudo-rapidity of $|\eta|<2.0$.

The distribution of the leading track $p_{T}^{t r}$ is shown in figure 5.12. Only events with an isolated quality track are shown. The remaining Standard Model backgrounds mainly

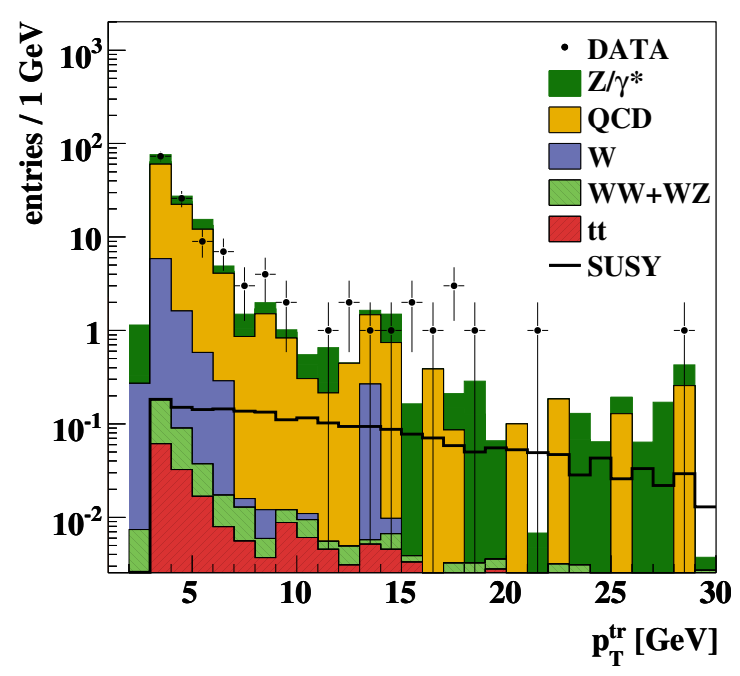

Figure 5.12: Transverse momentum $p_{T}$ of the selected charged track.

consist of QCD and $Z / \gamma^{*}$. A small amount of events from QCD sources fulfill the requirement of an isolated quality track and pass the selection. This track is most probably a third muon in the event, originating from a b-jet. The third track in the $Z / \gamma^{*}$ events arises from gluon radiation in the initial state (initial state radiation).

For the SUSY signal only $60 \%$ of the events pass this selection, that means the events have an additional isolated track. It is the most restrictive selection of all, for the SUSY signal events. Softening the quality requirements necessarily allows more events from background sources to pass the cut.

Events pass this selection if the isolated track has a transverse momentum of

$$
p_{T}^{t r}>3 \mathrm{GeV}
$$

Events from the $\mathrm{W}$ background have a large amount of $\mathbb{E}_{T}$ and could have been a large background after the previous selection cut (on $\mathbb{E}_{T}$ ). This background is reduced significantly, with the requirement of the third track in the event. 


\subsubsection{Invariant Mass: $M_{\mu t r}$}

The two selected muons are the leading- $p_{T}$ muons in the event. The third track in the event, could also be a muon track. Therefore the invariant mass of such a track and one of the muons can give the $\mathrm{Z}$ boson mass. Similar to $M_{\mu \mu}$, the reconstructed invariant mass from one of the muons $\mu_{1}$ or $\mu_{2}$ and the selected track is determined like in equation 5.1 and shown in figure $5.13 \mathrm{a}), \mathrm{b}$ ).
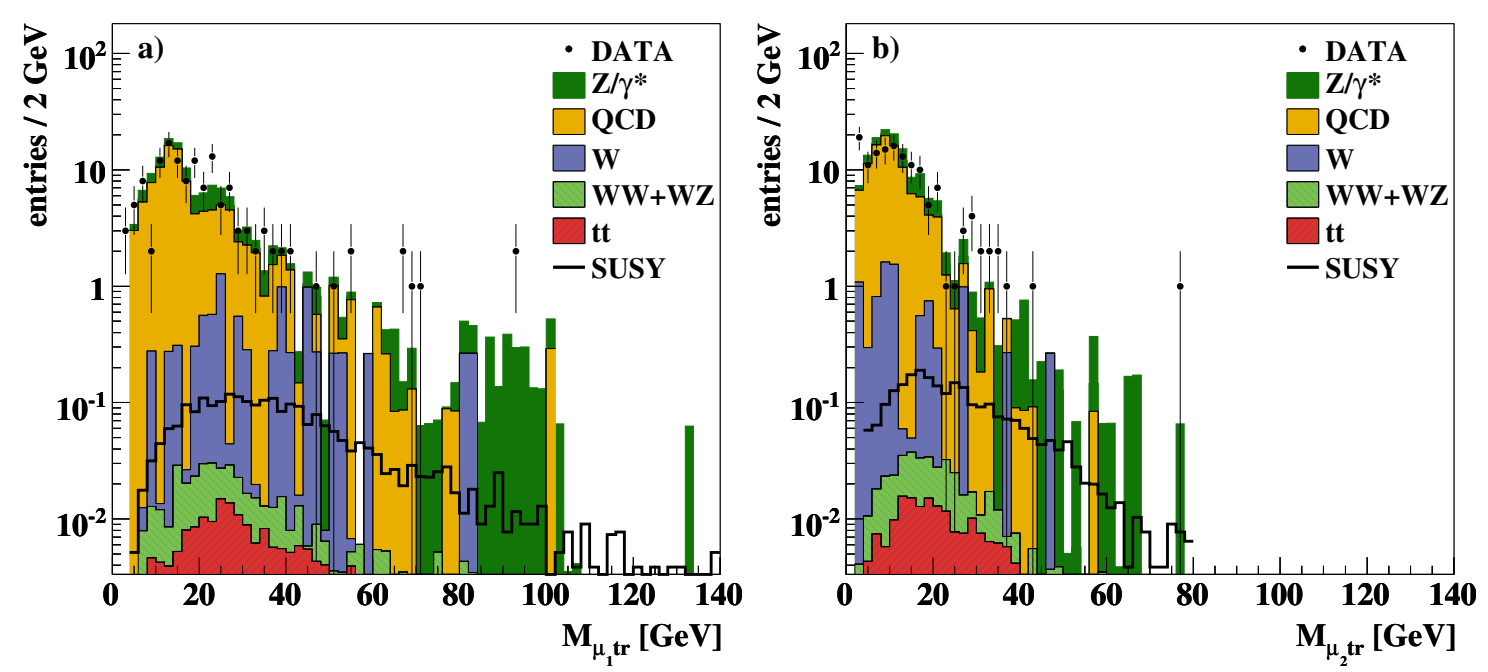

Figure 5.13: The invariant mass from the first (second) muon and selected track.

The sum of the remaining Standard Model background events is compared with the SUSY signal and the data events. There are events from the $Z / \gamma^{*}$ background with a reconstructed mass of $\mu_{1}$ or $\mu_{2}$ and the track in the region of the $Z$ resonance. These are events with three muons, where one of the previously selected muons does not originate from the direct $Z \rightarrow \mu^{+} \mu^{-}$decay.

The SUSY signal has small invariant masses. The invariant mass is bound at high masses, because of the mass difference of the gauginos.

The selection cut is set to reject events similar to the $\mathrm{Z}$ boson mass. Events with

$$
M_{\mu_{1} t r}<70 \mathrm{GeV}
$$

pass the selection. No cut is applied on the invariant mass from the track and the second muon. This cut is mainly efficient for the $Z \rightarrow \mu^{+} \mu^{-}$background. It removes events where the selected track is not identified as the muon from the $Z \rightarrow \mu^{+} \mu^{-}$decay. 


\subsection{6 $p_{T}$-Balance: $\Sigma_{p_{T}} / p_{T}^{t r}$}

In a SUSY signal like event, the vectorial sum of the transverse momenta of the two selected muons and the $\mathbb{E}_{T}$ should be equal to the transverse momentum of the selected third track $p_{T}^{t r}$. This is true for all events which do not feature a jet in the event and the $\mathbb{E}_{T}$ is not mismeasured. A quantity $\Sigma_{p_{T}} / p_{T}^{t r}$ is then calculated and referred to as $p_{T}$-balance:

$$
\Sigma_{p_{T}} / p_{T}^{t r}=\frac{p_{T}^{\mu_{1}}+p_{T}^{\mu_{2}}+\mathbb{E}_{T}}{p_{T}^{t r}} .
$$

The momenta of the two muons and the missing energy are added (vectorial sum) and the resulting sum is divided by the transverse momentum of the selected track $p_{T}^{t r}$.

The sum of the Standard Model backgrounds and the SUSY signal are shown in figure $5.14 \mathrm{a}$ ), b), respectively. The SUSY signal shows a peak at the value 1.0, whereas the sum of the backgrounds show a small contribution in this region. Especially for the QCD events, which have jets in the final state, this selection variable shows mainly a contribution for a value above 3.0 .
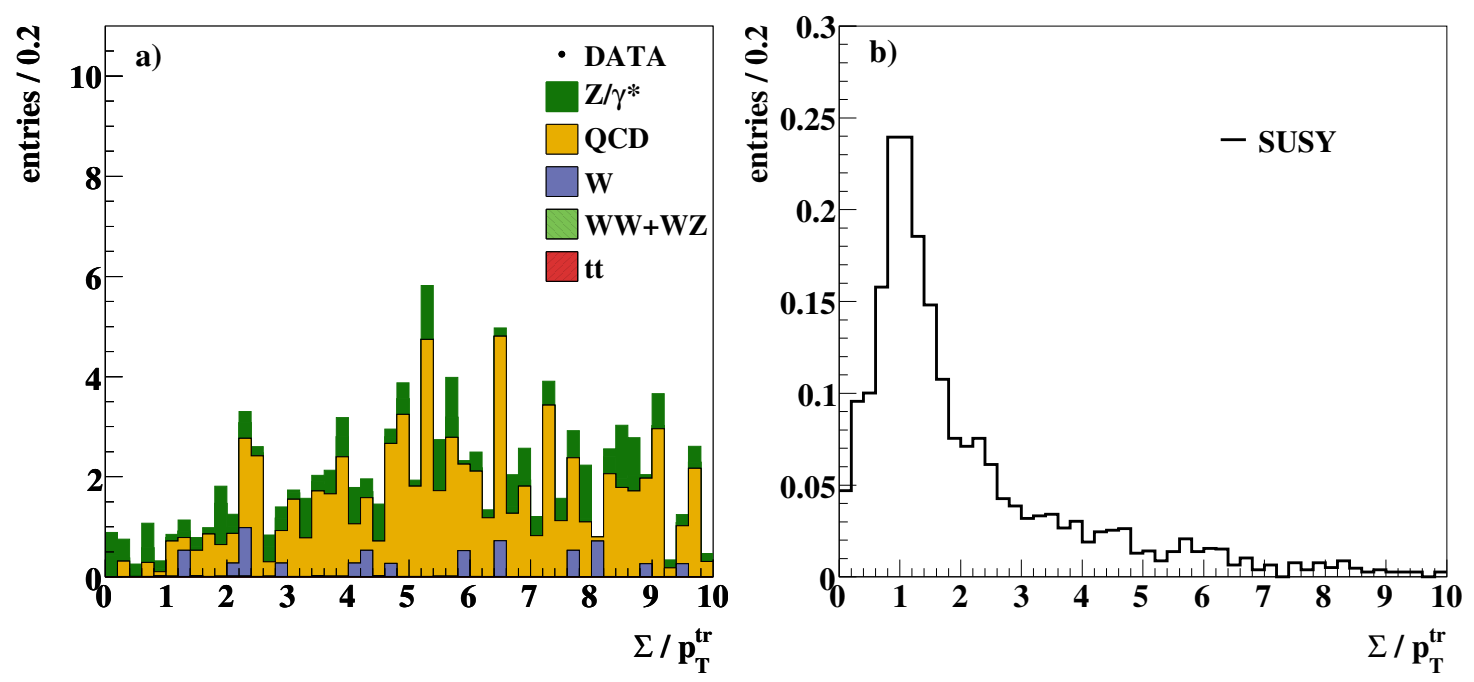

Figure 5.14: The $p_{T}$-balance for the Standard Model backgrounds (left) and the SUSY signal (right).

The same distribution is shown in figure 5.15 in a logarithmic scale. The sum of all Standard Model backgrounds is compared with the data events and the SUSY signal. Here it can be seen more clearly, that the $Z / \gamma^{*}$ events contribute with low values for $\Sigma_{p_{T}} / p_{T}^{t r}$. These are events with a mismeasured $\mathbb{E}_{T}$ or a mismeasured muon transverse momentum. A cut is applied at

$$
0.3<\Sigma_{p_{T}} / p_{T}^{t r}<3.0
$$

This cut rejects $90 \%$ of the QCD events left from the previous selection cuts. Its effect on the other remaining main backgrounds, already heavily reduced and with low statistics at this stage of the event selection, is similarly. 


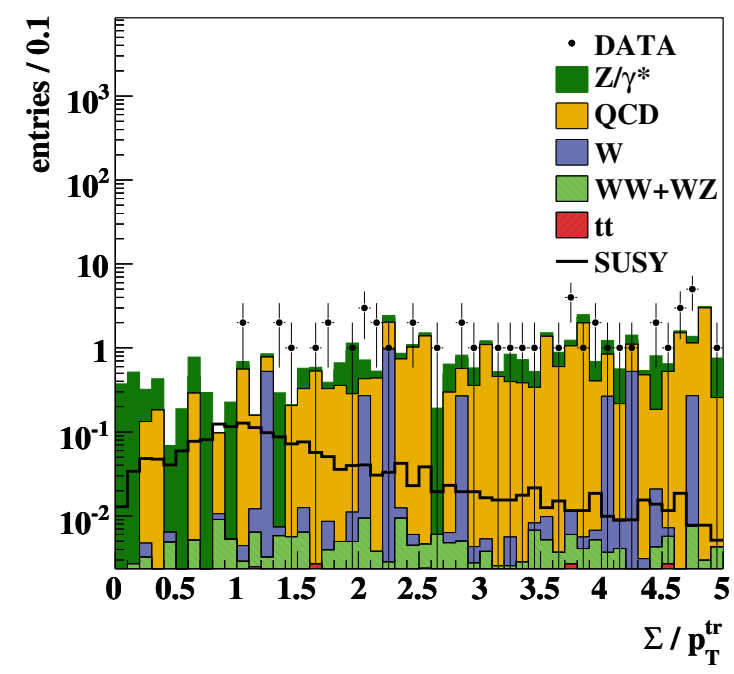

Figure 5.15: The $p_{T}$-balance distribution.

\subsubsection{Transverse Mass $M_{T}$ and $\operatorname{Sig}\left(\mathbb{E}_{T}\right)$ :}

Large values of $\mathbb{E}_{T}$ can arise from a poorly measured muon transverse momentum. In this case the angle between the muon and the $\mathbb{E}_{T}$ is small (both point in the same direction). This leads to small values for the transverse mass of the muon and the $\mathbb{E}_{T}$. The transverse mass $M_{T}$ between one of the muons and the $\mathbb{E}_{T}$ is calculated as:

$$
M_{T}=\sqrt{2 \mathbb{E}_{T} \cdot p_{T}^{\mu} \cdot\left(1-\cos \left(\Delta \phi\left(\mathbb{E}_{T}, p_{T}^{\mu}\right)\right)\right)}
$$

where $p_{T}^{\mu}$ is the transverse momentum of one of the muons $p_{T}^{\mu_{1}}$ or $p_{T}^{\mu_{2}}$.

Figure 5.16 a), b) shows the $M_{T}$ distributions for both muons respectively. The sum of the Standard Model background events consists mainly of QCD events for low $M_{T}$ and mainly Drell-Yan events for higher values. The minimum of the transverse mass of $M_{\mu_{1} t r}$ and $M_{\mu_{2} t r}$ is set to

$$
M_{T}>20 \mathrm{GeV} .
$$

Large values of $\mathbb{E}_{T}$ can be produced by the fluctuations of the energy of jets in the event. In this case the events are rejected if they contain jets with transverse energies above $15 \mathrm{GeV}$ and with a small significance $\operatorname{Sig}\left(\mathbb{E}_{T}\right)$

$$
\operatorname{Sig}\left(\mathscr{E}_{T}\right)>8 \sqrt{\mathrm{GeV}}
$$

(as described in section 4.5.3). The distribution for these events is shown in figure 5.17. The main background consists of QCD events. Events with two muons from $t \bar{t}$ processes, where a third isolated lepton from a b jet is reconstructed, can also be removed. 

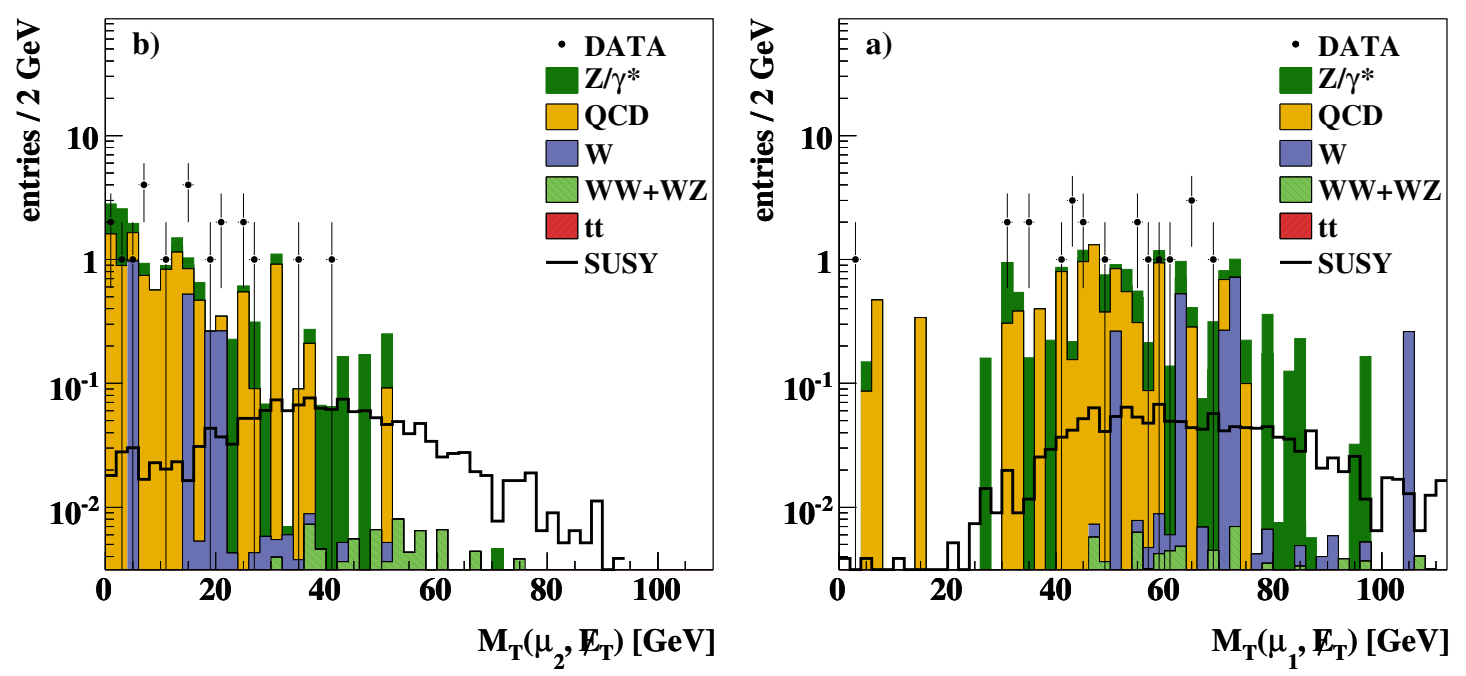

Figure 5.16: The transverse mass of first (second) muon and $\mathscr{E}_{T}$.

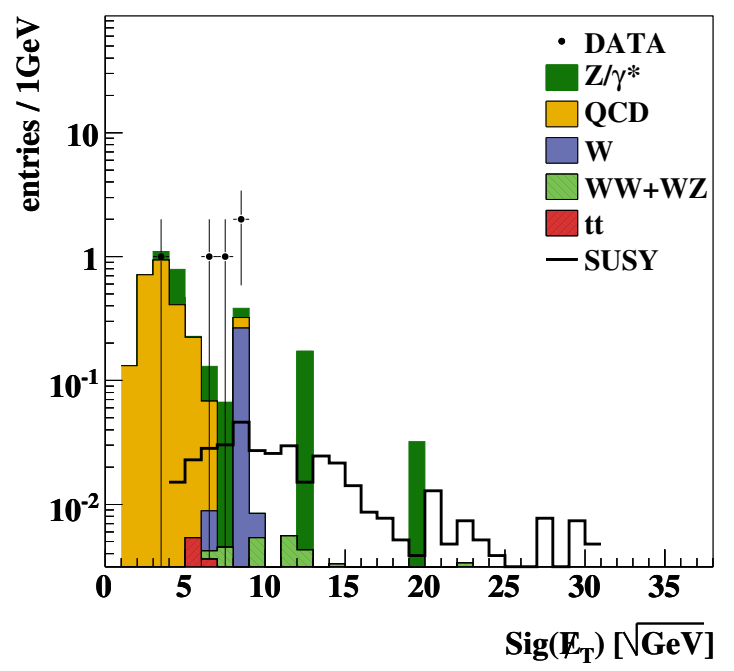

Figure 5.17: The scaled missing transverse energy for events with at least one jet with $E_{T}>15.0 \mathrm{GeV}$ (only events containing a jet are shown).

For the QCD background, both cuts $\left(M_{T}\right.$ and $\operatorname{Sig}\left(\mathbb{E}_{T}\right)$ ) are not applied explicitly. Only the reducing factors are applied on the QCD data. The reducing factors are obtained with higher statistics after the cut on the $\mathbb{E}_{T}$. These factors are not calculated from the antiisolated data. Instead the effect of the cut for the isolated data, after subtracting all but the QCD background, is used to calculate the reducing factors. At this early stage the potential effect of SUSY events is negligible. The same applies for the cut on $\operatorname{Sig}\left(\mathscr{E}_{T}\right)$.

The reason is, that the QCD background calculated from anti-isolated data, does not describe the data: both variables are dependent on the angle between the muon and the 
$\mathbb{E}_{T}$ or jets. This angle can be different for isolated and anti-isolated muons, also the jet activity in the event must not be the same for events with isolated and anti-isolated muons.

\subsubsection{Combination of $\mathbb{E}_{T}$ and Track: $p_{T}^{t r} \times \mathbb{E}_{T}$}

The last cut in this event selection combines the transverse momentum of the selected track with the $E_{T}$ in the event. The $Z / \gamma^{*}$ events are characterized by (small) values of $\mathbb{E}_{T}$ that pass its selection cut and have a track with high transverse momentum. The WW events have a large amount of $\mathbb{E}_{T}$, but low $p_{T}^{t r}$. SUSY signal events have a large amount of $\mathbb{E}_{T}$ and the $p_{T}^{t r}$ is higher as compared with the background events.

Figure 5.18 shows the distribution of $p_{T}^{t r} \times \mathbb{E}_{T}$ for the sum of the Standard Model background events, data and SUSY signal events. The statistics are very low at this stage

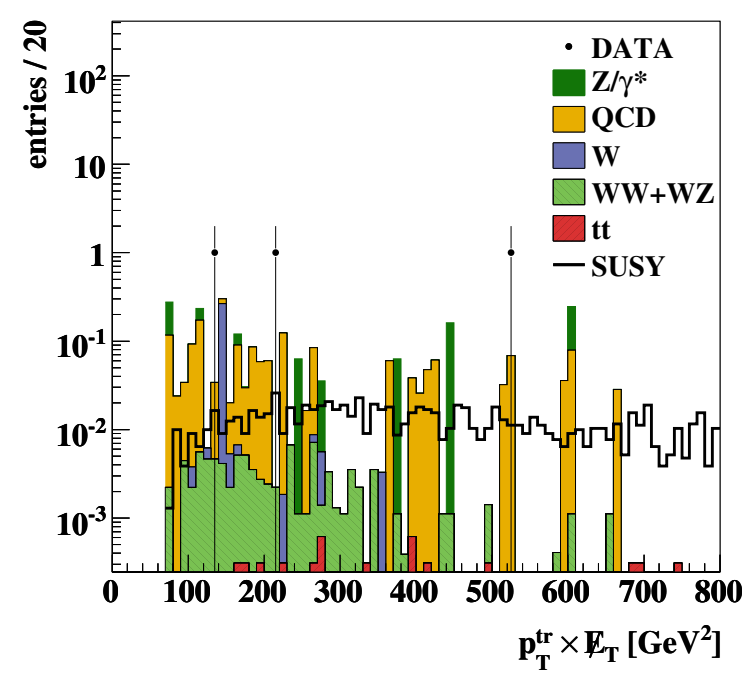

Figure 5.18: The combination of the transverse momentum of the track and $\mathbb{E}_{T}$.

of selection. Therefore this cut has been optimized using a different sample, which has been selected by lowering the cut on the leading- $p_{T}$ muon to be $p_{T}^{\mu_{1}}>9 \mathrm{GeV}$ and keeping all the other kinematic thresholds. The effect of the actual $p_{T}^{\mu_{1}}$ cut (at $11 \mathrm{GeV}$ ) however has been taken into account by the appropriate scale factor for all Monte Carlo samples.

The selection cut for $p_{T}^{t r} \times \mathbb{E}_{T}$ has been applied for several values and the resulting number of background and SUSY signal events have been used to calculate the expected limits on the cross section $\sigma \times B R(3 \ell)$ for the SUSY points D3 and C3. The expected cross section limit corresponds to a value for which the confidence level ([66]) drops below the $5 \%$ level.

Figure 5.19 shows the expected limit on the cross section with respect to the cut on the variable $p_{T}^{t r} \times \mathbb{E}_{T}$. The optimum cut was found at the minimum of the expected limit to be

$$
p_{T}^{t r} \times \mathbb{E}_{T}>150 \mathrm{GeV}^{2}
$$




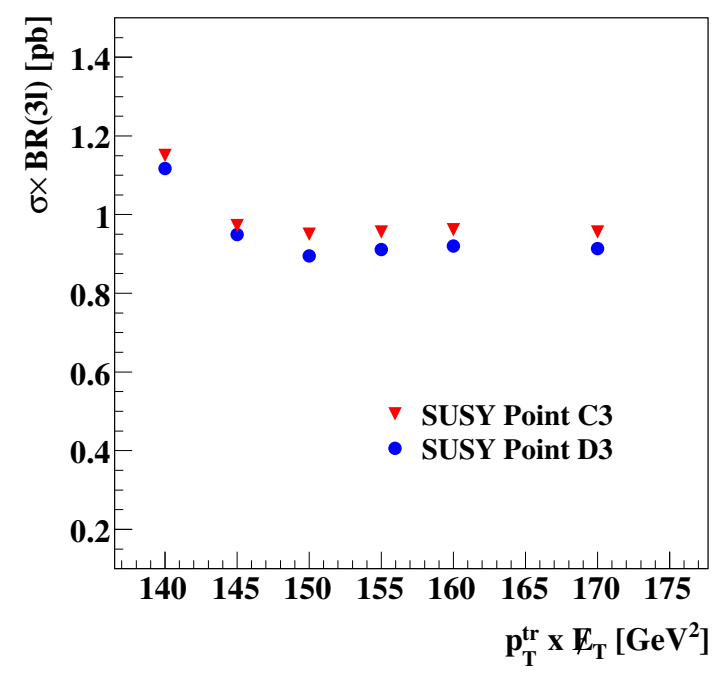

Figure 5.19: The expected limit on the cross section $\sigma \times B R(3 \ell)$ for several cuts on the variable $p_{T}^{t r} \times \mathbb{E}_{T}$ for SUSY point D3 (blue marker) and C3 (red marker).

Requiring $p_{T}^{t r} \times \mathbb{E}_{T}=150 \mathrm{GeV}^{2}$ corresponds to $p_{T}^{t r}=6.8 \mathrm{GeV}$ for events with $\mathbb{E}_{T}=$ $22 \mathrm{GeV}$, whereas for events with $\mathbb{E}_{T}>50 \mathrm{GeV}$ the $p_{T}^{t r}$ cut remains at $3.0 \mathrm{GeV}$.

After the last selection cut, only few events remain. The cut-flow table in the next section summarizes all selection variables and cuts and shows the number of events after each selection cut. 


\subsection{Comparison of Data and Background Events}

The chargino neutralino associated production has been investigated in events with two muons, an additional isolated track and missing transverse energy. A dedicated analysis has been performed and presented in the previous section. The event selection variables and cuts are resumed in table 5.2 starting with the preselection and followed by kinematic and topological selection variables.

\begin{tabular}{llc}
\hline \hline Selection variable & Cut name & Selection cut \\
\hline Preselection & Presel & muon quality \\
& & $p_{T}^{\mu_{1}}>9 \mathrm{GeV}$ \\
$p_{T}^{\mu_{2}}>5 \mathrm{GeV}$ \\
no cosmic muons \\
& & muons from primary vertex \\
& & muon isolation \\
\hline Transverse muon momentum & $p_{T}^{\mu_{1}}$ & $p_{T}^{\mu_{1}}>11 \mathrm{GeV}$ \\
Invariant dimuon mass & $M_{\mu \mu}$ & $15 \mathrm{GeV}<M_{\mu \mu}<50 \mathrm{GeV}$ \\
Missing transverse energy & $\mathbb{E}_{T}$ & $\mathbb{E}_{T}>22 \mathrm{GeV}$ \\
Isolated quality track & $p_{T}^{t r}$ & $p_{T}^{t r}>3 \mathrm{GeV}$ \\
Invariant mass of track+muon & $M_{\mu_{1} t r}$ & $M_{\mu_{1} t r}<70 \mathrm{GeV}$ \\
\hline$p_{T}$-balance & $\Sigma_{p_{T}} / p_{T}^{t r}$ & $0.3<\Sigma_{p_{T}} / p_{T}^{t r}<3.0$ \\
scaled $\mathscr{E}_{T}$ & $S_{i g}\left(\mathbb{E}_{T}\right)$ & $S i g\left(\mathbb{E}_{T}\right)>8 \sqrt{\mathrm{GeV}}$ \\
Transverse mass & $M_{T}$ & $M_{T}>20 \mathrm{GeV}$ \\
$\mathbb{E}_{T}$ and track & $p_{T}^{t r} \times \mathbb{E}_{T}$ & $p_{T}^{t r} \times \mathbb{E}_{T}>150 \mathrm{GeV}$ \\
\hline
\end{tabular}

Table 5.2: The selection variables in the order of the selection cuts applied.

The selection cuts have been applied successively to the events of the data and Monte Carlo simulation samples. The best combination of variables and cuts was chosen to discriminate best between the Standard Model background and the SUSY signal. The selection cuts were optimized to get the best expected cross section limit assuming that no signal will be observed.

The number of data events after each selection cut and the sum of the considered background events are shown in the first two columns of table 5.3 (upper half). The number of events observed in the data are in good agreement with the expected sum of events from the Standard Model background at all stages of the selection. The considered backgrounds are the Drell-Yan $\left(Z / \gamma^{*}\right)$, QCD, $t \bar{t}$ and di-boson processes. The errors listed in table 5.3 are statistical and systematic. The sources for the systematic uncertainty and the resulting relative error is described in section 5.5.

The $Z / \gamma^{*}$ background is greatly reduced after the application of the cuts on the dimuon mass of the selected isolated muons and the requirement of large missing transverse energy in the event. The remaining events contain an additional track originating from initial 


\begin{tabular}{|c|c|c|c|c|c|}
\hline Cut & Data & Sum background & $Z / \gamma^{*} \rightarrow \mu \mu$ & $Z / \gamma^{*} \rightarrow \tau \tau$ & QCD \\
\hline Presel & 40489 & $40362 \pm 818 \pm 3016$ & $29633 \pm 58 \pm 1036$ & $410 \pm 5 \pm 54$ & $10117 \pm 816 \pm 2052$ \\
\hline$p_{T}^{\mu_{1}}$ & 31649 & $30581 \pm 325 \pm 2571$ & $26022 \pm 50 \pm 1732$ & $382 \pm 5 \pm 52$ & $3976 \pm 321 \pm 941$ \\
\hline$M_{\mu \mu}$ & 12520 & $11749 \pm 319 \pm 638$ & $7418 \pm 36 \pm 489$ & $305 \pm 4 \pm 42$ & $3916 \pm 316 \pm 916$ \\
\hline $\mathbb{E}_{T}$ & 940 & $948 \pm 54 \pm 254$ & $162 \pm 5 \pm 15$ & $65 \pm 2 \pm 15$ & $632 \pm 54 \pm 238$ \\
\hline$p_{T}^{t r}$ & 139 & $137 \pm 11 \pm 40$ & $23 \pm 2 \pm 3$ & $7.66 \pm 0.70 \pm 2.26$ & $98 \pm 11 \pm 38$ \\
\hline$M_{\mu_{1} t r}$ & 135 & $134 \pm 11 \pm 40$ & $20 \pm 2 \pm 2$ & $7.60 \pm 0.70 \pm 2.23$ & $98 \pm 10 \pm 38$ \\
\hline$\Sigma_{\mathrm{p}_{\mathrm{T}}} / \mathrm{p}_{\mathrm{T}}^{\mathrm{tr}}$ & 21 & $16.75 \pm 2.00 \pm 5.10$ & $3.06 \pm 0.54 \pm 0.84$ & $2.27 \pm 0.39 \pm 0.56$ & $9.22 \pm 1.73 \pm 4.51$ \\
\hline $\operatorname{Sig}\left(\mathbb{E}_{T}\right)$ & 6 & $4.38 \pm 0.68 \pm 1.03$ & $1.10 \pm 0.38 \pm 0.24$ & $0.29 \pm 0.13 \pm 0.07$ & $2.57 \pm 0.48 \pm 1.04$ \\
\hline$M_{T}$ & 3 & $2.75 \pm 0.49 \pm 0.66$ & $0.56 \pm 0.25 \pm 0.10$ & $0.17 \pm 0.10 \pm 0.02$ & $1.64 \pm 0.31 \pm 0.67$ \\
\hline$p_{T}^{t r} \times \mathbb{E}_{T}$ & 2 & $1.75 \pm 0.34 \pm 0.46$ & $0.34 \pm 0.19 \pm 0.04$ & $0.17 \pm 0.10 \pm 0.02$ & $1.15 \pm 0.26 \pm 0.47$ \\
\hline Cut & & $t \bar{t} \rightarrow \mu^{ \pm} \vee \mu^{ \pm} \vee b \bar{b}$ & $W Z \rightarrow \mu \mu \nu \mu(e)$ & $W W \rightarrow \mu v \mu \nu$ & $W \rightarrow \mu \nu$ \\
\hline Presel & & $6.35 \pm 0.04$ & $1.36 \pm 0.01$ & $13.37 \pm 0.12$ & $131 \pm 6$ \\
\hline$p_{T}^{\mu_{1}}$ & & $6.34 \pm 0.04$ & $1.36 \pm 0.01$ & $13.35 \pm 0.12$ & $129 \pm 6$ \\
\hline$M_{\mu \mu}$ & & $1.45 \pm 0.02$ & $0.11 \pm 0.00$ & $3.79 \pm 0.06$ & $101 \pm 5$ \\
\hline $\mathbb{E}_{T}$ & & $1.34 \pm 0.02$ & $0.10 \pm 0.00$ & $3.24 \pm 0.06$ & $82 \pm 5$ \\
\hline$p_{T}^{t r}$ & & $0.19 \pm 0.01$ & $0.04 \pm 0.00$ & $0.22 \pm 0.01$ & $8.12 \pm 1.46$ \\
\hline$M_{\mu_{1} t r}$ & & $0.18 \pm 0.01$ & $0.01 \pm 0.00$ & $0.21 \pm 0.01$ & $7.60 \pm 1.41$ \\
\hline$\Sigma_{\mathrm{p}_{\mathrm{T}}} / \mathrm{p}_{\mathrm{T}}^{\mathrm{tr}}$ & & $0.03 \pm 0.00$ & $0.01 \pm 0.00$ & $0.09 \pm 0.01$ & $2.01 \pm 0.72$ \\
\hline $\operatorname{Sig}\left(\mathscr{E}_{T}\right)$ & & $0.03 \pm 0.00$ & $0.01 \pm 0.00$ & $0.08 \pm 0.01$ & $0.26 \pm 0.26$ \\
\hline$M_{T}$ & & $0.01 \pm 0.00$ & $0.01 \pm 0.00$ & $0.08 \pm 0.01$ & $0.26 \pm 0.26$ \\
\hline$p_{T}^{t r} \times \mathbb{E}_{T}$ & & $0.01 \pm 0.00$ & $0.01 \pm 0.00$ & $0.06 \pm 0.01$ & $0.00 \pm 0.00$ \\
\hline
\end{tabular}

Table 5.3: Number of data events and expected Monte Carlo background events after each selection cut. First the statistical, then the systematic errors are given in the upper half for the sum of all backgrounds, the Drell-Yan $Z / \gamma^{*}$ and QCD background. In the lower half only the statistical error is shown.

state radiation. The invariant mass of the track and one of the muons was tested whether it reconstructs the $\mathrm{Z}$ resonance and a cut was applied to remove those events.

The multi-jet backgrounds ( $t \bar{t}$ and QCD) are greatly reduced as the selected events have isolated muons, arising from $b$ decays, with small transverse momenta and a small amount of missing transverse energy in the event. Nevertheless further topological cuts have been applied to reject the remaining QCD events. Especially by applying the $p_{T^{-}}$ balance cut, events containing a jet or events where the $\mathbb{E}_{T}$ was mismeasured, could be rejected.

The WZ background is an irreducible physics background, but fortunately has a small cross section. Leptonic WW and $\mathrm{W}$ decays into final state with muons, have a large amount of $\mathbb{E}_{T}$ in the event, but are greatly reduced after the application of all cuts. The number of events after each selection cut is shown in table 5.3 (lower half). The errors are statistical.

Two candidate events are selected from the data sample after applying all selection cuts. The expected sum of events from the Standard Model background processes is $1.75 \pm 0.34$ (stat.) \pm 0.46 (syst.) events. The main contribution to the sum of background events is from QCD and Drell-Yan processes. The selected track in the remaining events is reconstructed from initial state radiation for the $Z / \gamma^{*}$ events. In the case of a QCD event, the track most probably arises from $b$ decays. The dominant errors are the statistical error 
of the QCD background and the systematic error of the QCD background modeling 2 .

No evidence for associated chargino neutralino production was found in the data sample with an integrated luminosity of $\sim 300 \mathrm{pb}^{-1}$. An upper limit for the cross section $\sigma \times B R(3 \ell)$ is determined and discussed in section 6.1. For this, the selection efficiencies for several SUSY points need to be known first.

\subsection{SUSY Points: Chargino and Slepton Mass Scan}

During the presentation of the event selection, an arbitrary SUSY point, D3 from table 4.1, was chosen to show the distribution of the SUSY signal compared to the expectations from Standard Model backgrounds. Nevertheless, all SUSY signal samples are subject to the same event selection. In total 36 combinations of the mSUGRA parameters have been considered. The mSUGRA parameters are such, that the chargino mass is above the LEP limit $m_{\widetilde{\chi}_{1}^{ \pm}}>103 \mathrm{GeV}[21]$.

A chargino mass scan can be performed from the SUSY signal samples: for a fixed mass difference $m_{\widetilde{\chi}_{2}^{0}}-m_{\tilde{\ell}}$ between the neutralino and the slepton, the chargino mass can have different values depending on the mSUGRA parameters. The same SUSY signal samples are also used to perform a slepton scan: for a fixed chargino mass, the mass difference $m_{\widetilde{\chi}_{2}^{0}}-m_{\tilde{\ell}}$ is varied. In the following the chargino mass scan is presented.

The chargino mass scan is discussed for three scenarios depending on the slepton mass:

- $m_{\widetilde{\chi}_{2}^{0}}-m_{\tilde{\ell}} \approx 10 \mathrm{GeV}(5 \mathrm{GeV})$

- $m_{\widetilde{\chi}_{2}^{0}} \approx m_{\tilde{\ell}} \quad$ and $\quad m_{\widetilde{\chi}_{2}^{0}}-m_{\tilde{\ell}} \approx-6 \mathrm{GeV}(-12 \mathrm{GeV})$

- $m_{\tilde{\ell}}$ is large

Tables 5.4-5.9 show the number of events after each selection cut for all SUSY points considered. The errors are given by the statistical and systematic error added in quadrature. The value of the mass difference $m_{\tilde{\chi}_{2}^{0}}-m_{\tilde{\ell}}$ is indicated in each table, as well as the production cross section for each SUSY point along with the chargino mass.

With increasing slepton mass, the cross section decreases, if the chargino mass is the same, e.g. the cross section is decreasing from A1-A6 shown in tables 5.4-5.9. If the mass difference $\left|m_{\widetilde{\chi}_{2}^{0}, \widetilde{\chi}_{1}^{ \pm}}-m_{\tilde{\ell}}\right|$ is large enough, also the $p_{T}$ 's of the leptons from the $\widetilde{\chi}_{1}^{ \pm} \widetilde{\chi}_{2}^{0}$ decays are large enough to be detected.

In the first scenario, the mass of the slepton $m_{\tilde{\ell}}$ is smaller than the masses of the $\widetilde{\chi}_{2}^{0}$ and $\widetilde{\chi}_{1}^{ \pm}$. These can then decay via on-shell sleptons. The mass difference between the gauginos and the slepton is crucial for the selection efficiency.

The production cross section for the SUSY points in table 5.4 decreases with increasing $m_{\tilde{\chi}_{1}^{ \pm}}$(e.g. from A1 to F1). The mass difference is $m_{\tilde{\chi}_{2}^{0}} \approx m_{\tilde{\ell}}+10 \mathrm{GeV}$ for these SUSY

\footnotetext{
${ }^{2}$ See also section 5.5 for details of the systematic uncertainty.
} 
points. The selection efficiency for the preselection relative to the decay channel with at least two muons $\left(\widetilde{\chi}_{1}^{ \pm} \widetilde{\chi}_{2}^{0} \rightarrow \mu \mu \ell \vee \widetilde{\chi}_{1}^{0} \widetilde{\chi}_{1}^{0}\right)$, is $22-26 \%$ and decreases to $3 \%$ after all cuts. If the mass difference $m_{\widetilde{\chi}_{2}^{0}}-m_{\tilde{\ell}}$ gets as small as $5 \mathrm{GeV}$ (table 5.5) the slepton is produced together with a lepton having very low transverse momentum. The selection of such a lepton is difficult and leads to a decrease in the selection efficiency to $14-18 \%$ (relative to the decay channel with at least two muons) already at the early stage of preselection. After all cuts the selection efficiency is $1-2 \%$ for the SUSY points A2-F2.

\begin{tabular}{|c|c|c|c|c|c|c|}
\hline & \multicolumn{6}{|c|}{$m_{\tilde{\chi}_{1}^{ \pm}}$scan with $m_{\widetilde{\chi}_{2}^{0}}-m_{\tilde{\ell}} \approx+10 \mathrm{GeV}$} \\
\hline & A1 & $\mathrm{B} 1$ & $\mathrm{C} 1$ & D1 & E1 & $\mathrm{F} 1$ \\
\hline$\sigma \times B R$ & $1.03 \mathrm{pb}$ & $0.86 \mathrm{pb}$ & $0.73 \mathrm{pb}$ & $0.60 \mathrm{pb}$ & $0.51 \mathrm{pb}$ & $0.26 \mathrm{pb}$ \\
\hline$m_{\widetilde{\chi}_{1}^{ \pm}}$ & $98 \mathrm{GeV}$ & $101 \mathrm{GeV}$ & $106 \mathrm{GeV}$ & $110 \mathrm{GeV}$ & $114 \mathrm{GeV}$ & $132 \mathrm{GeV}$ \\
\hline Cut & & & & & & \\
\hline Presel & $20.78 \pm 2.40$ & $17.58 \pm 2.03$ & $15.80 \pm 1.82$ & $13.67 \pm 1.57$ & $11.73 \pm 1.34$ & $5.91 \pm 0.68$ \\
\hline$p_{T}^{\mu_{1}}$ & $20.37 \pm 2.35$ & $17.30 \pm 2.00$ & $15.57 \pm 1.79$ & $13.46 \pm 1.55$ & $11.61 \pm 1.33$ & $5.87 \pm 0.68$ \\
\hline$M_{\mu \mu}$ & $19.19 \pm 2.22$ & $16.14 \pm 1.87$ & $14.52 \pm 1.67$ & $12.58 \pm 1.45$ & $10.64 \pm 1.22$ & $5.23 \pm 0.61$ \\
\hline $\mathscr{E}_{T}$ & $14.55 \pm 1.69$ & $12.28 \pm 1.43$ & $11.19 \pm 1.29$ & $9.66 \pm 1.11$ & $8.40 \pm 0.97$ & $4.36 \pm 0.51$ \\
\hline$p_{T}^{t r}$ & $7.86 \pm 0.93$ & $6.83 \pm 0.81$ & $5.87 \pm 0.69$ & $5.27 \pm 0.62$ & $4.68 \pm 0.54$ & $2.63 \pm 0.31$ \\
\hline$M_{\mu_{1} t r}$ & $7.31 \pm 0.87$ & $6.30 \pm 0.75$ & $5.40 \pm 0.64$ & $4.70 \pm 0.55$ & $4.12 \pm 0.48$ & $2.23 \pm 0.27$ \\
\hline$\Sigma_{\mathrm{p}_{\mathrm{T}}} / \mathrm{p}_{\mathrm{T}}^{\mathrm{rr}}$ & $4.57 \pm 0.55$ & $3.91 \pm 0.48$ & $3.30 \pm 0.40$ & $2.90 \pm 0.34$ & $2.44 \pm 0.29$ & $1.38 \pm 0.17$ \\
\hline $\operatorname{Sig}\left(\mathbb{E}_{T}\right)$ & $3.33 \pm 0.42$ & $2.99 \pm 0.37$ & $2.35 \pm 0.29$ & $2.19 \pm 0.26$ & $1.90 \pm 0.23$ & $1.03 \pm 0.13$ \\
\hline$M_{T}$ & $2.91 \pm 0.36$ & $2.56 \pm 0.32$ & $2.06 \pm 0.25$ & $1.86 \pm 0.23$ & $1.62 \pm 0.19$ & $0.90 \pm 0.11$ \\
\hline$p_{T}^{t r} \times \mathbb{E}_{T}$ & $2.72 \pm 0.34$ & $2.38 \pm 0.30$ & $1.91 \pm 0.24$ & $1.77 \pm 0.22$ & $1.52 \pm 0.18$ & $0.87 \pm 0.11$ \\
\hline
\end{tabular}

Table 5.4: Number of events after each selection cut for the chargino scan A1 - F1.

\begin{tabular}{|c|c|c|c|c|c|c|}
\hline & \multicolumn{6}{|c|}{$m_{\tilde{\chi}_{1}^{ \pm}}$scan with $m_{\widetilde{\chi}_{2}^{0}}-m_{\tilde{\ell}} \approx+5 \mathrm{GeV}$} \\
\hline & $\mathrm{A} 2$ & B2 & $\mathrm{C} 2$ & D2 & E2 & F2 \\
\hline$\sigma \times B R$ & $0.77 \mathrm{pb}$ & $0.66 \mathrm{pb}$ & $0.55 \mathrm{pb}$ & $0.47 \mathrm{pb}$ & $0.40 \mathrm{pb}$ & $0.18 \mathrm{pb}$ \\
\hline$m_{\widetilde{\chi}_{1}^{ \pm}}$ & $98 \mathrm{GeV}$ & $101 \mathrm{GeV}$ & $106 \mathrm{GeV}$ & $110 \mathrm{GeV}$ & $114 \mathrm{GeV}$ & $132 \mathrm{GeV}$ \\
\hline \multicolumn{7}{|l|}{ Cut } \\
\hline Presel & $9.97 \pm 1.16$ & $8.87 \pm 1.03$ & $7.65 \pm 0.89$ & $7.01 \pm 0.81$ & $6.39 \pm 0.74$ & $2.69 \pm 0.31$ \\
\hline$p_{T}^{\mu_{1}}$ & $9.75 \pm 1.13$ & $8.76 \pm 1.02$ & $7.53 \pm 0.87$ & $6.93 \pm 0.80$ & $6.31 \pm 0.73$ & $2.66 \pm 0.31$ \\
\hline$M_{\mu \mu}$ & $7.61 \pm 0.89$ & $7.09 \pm 0.83$ & $6.04 \pm 0.70$ & $5.63 \pm 0.65$ & $5.14 \pm 0.60$ & $1.81 \pm 0.21$ \\
\hline $\mathscr{E}_{T}$ & $5.79 \pm 0.68$ & $5.55 \pm 0.65$ & $4.72 \pm 0.55$ & $4.44 \pm 0.52$ & $4.17 \pm 0.49$ & $1.51 \pm 0.18$ \\
\hline$p_{T}^{t r}$ & $3.37 \pm 0.41$ & $2.95 \pm 0.36$ & $2.69 \pm 0.32$ & $2.38 \pm 0.29$ & $2.33 \pm 0.28$ & $0.84 \pm 0.10$ \\
\hline$M_{\mu_{1} t r}$ & $3.02 \pm 0.37$ & $2.66 \pm 0.32$ & $2.41 \pm 0.29$ & $2.14 \pm 0.26$ & $1.99 \pm 0.24$ & $0.69 \pm 0.08$ \\
\hline$\Sigma_{\mathrm{p}_{\mathrm{T}}} / \mathrm{p}_{\mathrm{T}}^{\mathrm{tr}}$ & $1.79 \pm 0.23$ & $1.59 \pm 0.20$ & $1.39 \pm 0.17$ & $1.24 \pm 0.15$ & $1.22 \pm 0.15$ & $0.43 \pm 0.05$ \\
\hline $\operatorname{Sig}\left(\mathbb{E}_{T}\right)$ & $1.21 \pm 0.16$ & $1.08 \pm 0.14$ & $0.91 \pm 0.12$ & $0.81 \pm 0.10$ & $0.83 \pm 0.10$ & $0.33 \pm 0.04$ \\
\hline$M_{T}$ & $1.10 \pm 0.15$ & $0.99 \pm 0.13$ & $0.78 \pm 0.10$ & $0.70 \pm 0.09$ & $0.77 \pm 0.10$ & $0.32 \pm 0.04$ \\
\hline$p_{T}^{t r} \times \mathbb{E}_{T}^{t}$ & $0.99 \pm 0.14$ & $0.86 \pm 0.11$ & $0.68 \pm 0.09$ & $0.65 \pm 0.08$ & $0.70 \pm 0.09$ & $0.30 \pm 0.04$ \\
\hline
\end{tabular}

Table 5.5: Number of events after each selection cut for the chargino scan A2 - F2. 
After all selection cuts the numbers of selected SUSY events for the scenario where gauginos are heavier than the sleptons, range from $2.72 \pm 0.34$ for SUSY point A1 to $0.3 \pm 0.04$ for SUSY point F2.

The highest selection efficiency is obtained for the SUSY points with $m_{\widetilde{\chi}_{2}^{0}} \approx m_{\tilde{\ell}}$ shown in table 5.6. If the neutralino and slepton mass is nearly equal, or the slepton mass is only slightly heavier than the neutralino mass, the decays via virtual sleptons is enhanced. The leptons have large $p_{T}$ 's and can be detected. The selection efficiency for the preselection is $40 \%$ for point A3 rising to $46 \%$ for point F3. The production cross section decreases with increasing chargino mass (A3 - F3). The selection efficiency is calculated relative to the decay channel with at least two muons.

\begin{tabular}{|c|c|c|c|c|c|c|}
\hline \multicolumn{7}{|c|}{$m_{\tilde{\chi}_{1}^{ \pm}}$scan with $m_{\tilde{\chi}_{2}^{0}}=m_{\tilde{\ell}}$} \\
\hline & A3 & B3 & $\mathrm{C} 3$ & D3 & E3 & F3 \\
\hline$\sigma \times B R$ & $0.39 \mathrm{pb}$ & $0.32 \mathrm{pb}$ & $0.26 \mathrm{pb}$ & $0.21 \mathrm{pb}$ & $0.18 \mathrm{pb}$ & $0.07 \mathrm{pb}$ \\
\hline$m_{\widetilde{\chi}_{1}^{ \pm}}$ & $98 \mathrm{GeV}$ & $101 \mathrm{GeV}$ & $106 \mathrm{GeV}$ & $110 \mathrm{GeV}$ & $114 \mathrm{GeV}$ & $132 \mathrm{GeV}$ \\
\hline \multicolumn{7}{|l|}{ Cut } \\
\hline Presel & $13.88 \pm 1.59$ & $11.52 \pm 1.32$ & $9.81 \pm 1.12$ & $7.83 \pm 0.90$ & $6.62 \pm 0.76$ & $3.03 \pm 0.35$ \\
\hline$p_{T}^{\mu_{1}}$ & $13.72 \pm 1.58$ & $11.40 \pm 1.30$ & $9.71 \pm 1.11$ & $7.77 \pm 0.89$ & $6.57 \pm 0.75$ & $3.02 \pm 0.35$ \\
\hline$M_{\mu \mu}$ & $11.50 \pm 1.32$ & $9.47 \pm 1.08$ & $7.94 \pm 0.91$ & $6.33 \pm 0.73$ & $5.13 \pm 0.59$ & $2.03 \pm 0.23$ \\
\hline $\mathbb{E}_{T}$ & $8.35 \pm 0.96$ & $6.98 \pm 0.80$ & $5.97 \pm 0.69$ & $4.92 \pm 0.57$ & $4.06 \pm 0.47$ & $1.69 \pm 0.19$ \\
\hline$p_{T}^{t r}$ & $4.73 \pm 0.55$ & $4.00 \pm 0.46$ & $3.45 \pm 0.40$ & $2.74 \pm 0.32$ & $2.43 \pm 0.28$ & $1.03 \pm 0.12$ \\
\hline$M_{\mu_{1} t r}$ & $4.34 \pm 0.51$ & $3.61 \pm 0.42$ & $3.05 \pm 0.35$ & $2.41 \pm 0.28$ & $2.18 \pm 0.25$ & $0.87 \pm 0.10$ \\
\hline$\Sigma_{\mathrm{p}_{\mathrm{T}}} / \mathrm{p}_{\mathrm{T}}^{\mathrm{tr}}$ & $2.96 \pm 0.35$ & $2.44 \pm 0.28$ & $2.10 \pm 0.25$ & $1.67 \pm 0.19$ & $1.49 \pm 0.17$ & $0.62 \pm 0.07$ \\
\hline $\operatorname{Sig}\left(\mathbb{E}_{T}\right)$ & $2.36 \pm 0.28$ & $1.93 \pm 0.23$ & $1.69 \pm 0.20$ & $1.36 \pm 0.16$ & $1.23 \pm 0.15$ & $0.52 \pm 0.06$ \\
\hline$M_{T}$ & $2.10 \pm 0.25$ & $1.77 \pm 0.21$ & $1.50 \pm 0.18$ & $1.26 \pm 0.15$ & $1.13 \pm 0.13$ & $0.48 \pm 0.06$ \\
\hline$p_{T}^{t r} \times \mathbb{E}_{T}$ & $1.93 \pm 0.23$ & $1.67 \pm 0.20$ & $1.43 \pm 0.17$ & $1.20 \pm 0.14$ & $1.08 \pm 0.13$ & $0.47 \pm 0.06$ \\
\hline
\end{tabular}

Table 5.6: Number of events after each selection cut for the chargino scan A3 - F3.

After all cuts, the selection efficiency is 6-7\%. The limit on the cross section is presented in section 6.1 for these SUSY points as an example for the sensitivity of the investigated decay channel.

For SUSY points with higher slepton masses, the leptonic branching fraction decreases compared to the SUSY points with enhanced leptonic branching fraction. The tables 5.7 and 5.8 show the number of events after each cut for SUSY points, where the mass difference between the neutralino and slepton is $6 \mathrm{GeV}$ and $12 \mathrm{GeV}$. The selection efficiency of 30-34\% for the preselection is still higher than for the SUSY points where gauginos decay via real sleptons. After all cuts the selection efficiency is 4-5\%. The selection efficiency is calculated relative to the decay channel with at least two muons.

For large slepton masses, here as large as $m_{\widetilde{\chi}_{2}^{0}}+100 \mathrm{GeV}$, the $\widetilde{\chi}_{1}^{ \pm} \widetilde{\chi}_{2}^{0}$ decay into leptons via virtual bosons, as the decay via sleptons is suppressed. Table 5.9 shows the number of events after each selection cut. The efficiency after preselection is 33-36\% and decreases to $5 \%$ after all cuts. These SUSY points are shown as a reference. The $\sigma \times B R(3 \ell)$ is $80 \%$ smaller than for the SUSY points with larger leptonic branching fraction. 


\begin{tabular}{|c|c|c|c|c|c|c|}
\hline \multicolumn{7}{|c|}{$m_{\tilde{\chi}_{1}^{ \pm}}$scan with $m_{\widetilde{\chi}_{2}^{0}}-m_{\tilde{\ell}}=-6 \mathrm{GeV}$} \\
\hline & A4 & B4 & $\mathrm{C} 4$ & D4 & E4 & F4 \\
\hline$\sigma \times B R$ & $0.35 \mathrm{pb}$ & $0.28 \mathrm{pb}$ & $0.23 \mathrm{pb}$ & $0.19 \mathrm{pb}$ & $0.15 \mathrm{pb}$ & $0.07 \mathrm{pb}$ \\
\hline$m_{\tilde{\chi}_{1}^{ \pm}}$ & $98 \mathrm{GeV}$ & $101 \mathrm{GeV}$ & $106 \mathrm{GeV}$ & $110 \mathrm{GeV}$ & $114 \mathrm{GeV}$ & $132 \mathrm{GeV}$ \\
\hline \multicolumn{7}{|l|}{ Cut } \\
\hline Presel & $9.27 \pm 1.07$ & $7.93 \pm 0.91$ & $6.51 \pm 0.75$ & $5.45 \pm 0.62$ & $4.53 \pm 0.52$ & $2.14 \pm 0.25$ \\
\hline$p_{T}^{\mu_{1}}$ & $9.19 \pm 1.06$ & $7.87 \pm 0.90$ & $6.45 \pm 0.74$ & $5.40 \pm 0.62$ & $4.49 \pm 0.52$ & $2.13 \pm 0.24$ \\
\hline$M_{u \mu}$ & $7.94 \pm 0.92$ & $6.74 \pm 0.78$ & $5.37 \pm 0.62$ & $4.32 \pm 0.50$ & $3.51 \pm 0.40$ & $1.43 \pm 0.17$ \\
\hline$\ddot{E}_{T}$ & $5.81 \pm 0.67$ & $4.80 \pm 0.55$ & $3.95 \pm 0.45$ & $3.25 \pm 0.37$ & $2.68 \pm 0.31$ & $1.17 \pm 0.13$ \\
\hline$p_{T}^{t r}$ & $3.30 \pm 0.39$ & $2.83 \pm 0.33$ & $2.31 \pm 0.27$ & $1.87 \pm 0.22$ & $1.61 \pm 0.19$ & $0.72 \pm 0.08$ \\
\hline$M_{\mu_{1} t r}$ & $2.99 \pm 0.35$ & $2.57 \pm 0.30$ & $2.11 \pm 0.25$ & $1.65 \pm 0.19$ & $1.38 \pm 0.16$ & $0.60 \pm 0.07$ \\
\hline$\Sigma_{\mathrm{p}_{\mathrm{T}}} / \mathrm{p}_{\mathrm{T}}^{\mathrm{tr}}$ & $2.05 \pm 0.25$ & $1.77 \pm 0.21$ & $1.47 \pm 0.17$ & $1.13 \pm 0.13$ & $0.94 \pm 0.11$ & $0.43 \pm 0.05$ \\
\hline $\operatorname{Sig}\left(\mathbb{E}_{T}\right)$ & $1.68 \pm 0.20$ & $1.43 \pm 0.17$ & $1.17 \pm 0.14$ & $0.92 \pm 0.11$ & $0.78 \pm 0.09$ & $0.36 \pm 0.05$ \\
\hline$M_{T}$ & $1.52 \pm 0.19$ & $1.29 \pm 0.15$ & $1.03 \pm 0.12$ & $0.83 \pm 0.10$ & $0.73 \pm 0.09$ & $0.32 \pm 0.04$ \\
\hline$p_{T}^{t r} \times \mathbb{E}_{T}$ & $1.43 \pm 0.18$ & $1.23 \pm 0.15$ & $0.97 \pm 0.11$ & $0.81 \pm 0.10$ & $0.71 \pm 0.08$ & $0.31 \pm 0.04$ \\
\hline
\end{tabular}

Table 5.7: Number of events after each selection cut for the chargino scan A4 - F4.

\begin{tabular}{ccccccc}
\hline \hline & \multicolumn{7}{c}{$m_{\tilde{\chi}_{1}^{ \pm}}$scan with $m_{\tilde{\chi}_{2}^{0}}-m_{\tilde{\ell}}=-12 \mathrm{GeV}$} \\
& $\mathrm{A} 5$ & $\mathrm{~B} 5$ & $\mathrm{C} 5$ & $\mathrm{D} 5$ & $\mathrm{E} 5$ & $\mathrm{~F} 5$ \\
\cline { 2 - 7 }$\sigma \times B R$ & $0.29 \mathrm{pb}$ & $0.24 \mathrm{pb}$ & $0.19 \mathrm{pb}$ & $0.15 \mathrm{pb}$ & $0.13 \mathrm{pb}$ & $0.06 \mathrm{pb}$ \\
$m_{\tilde{\chi}_{1}^{ \pm}}$ & $98 \mathrm{GeV}$ & $101 \mathrm{GeV}$ & $106 \mathrm{GeV}$ & $110 \mathrm{GeV}$ & $114 \mathrm{GeV}$ & $132 \mathrm{GeV}$ \\
\hline Cut & \multicolumn{7}{c}{} & & \\
\hline Presel & $8.22 \pm 0.95$ & $6.91 \pm 0.79$ & $5.80 \pm 0.66$ & $4.75 \pm 0.54$ & $3.76 \pm 0.43$ & $1.88 \pm 0.21$ \\
$p_{T}^{\mu_{1}}$ & $8.12 \pm 0.93$ & $6.79 \pm 0.78$ & $5.76 \pm 0.66$ & $4.70 \pm 0.54$ & $3.73 \pm 0.43$ & $1.87 \pm 0.21$ \\
$M_{\mu \mu}$ & $7.00 \pm 0.81$ & $5.79 \pm 0.67$ & $4.80 \pm 0.55$ & $3.72 \pm 0.43$ & $2.94 \pm 0.34$ & $1.28 \pm 0.15$ \\
$\mathbb{E}_{T}$ & $4.91 \pm 0.57$ & $4.22 \pm 0.49$ & $3.54 \pm 0.41$ & $2.93 \pm 0.34$ & $2.24 \pm 0.26$ & $1.03 \pm 0.12$ \\
$p_{T}^{t r}$ & $2.83 \pm 0.33$ & $2.45 \pm 0.28$ & $2.10 \pm 0.24$ & $1.69 \pm 0.20$ & $1.37 \pm 0.16$ & $0.63 \pm 0.07$ \\
$M_{\mu_{1} t r}$ & $2.56 \pm 0.30$ & $2.19 \pm 0.26$ & $1.90 \pm 0.22$ & $1.39 \pm 0.16$ & $1.19 \pm 0.14$ & $0.53 \pm 0.06$ \\
$\Sigma_{\mathrm{pT}} / p_{\mathrm{T}}^{\mathrm{tr}}$ & $1.72 \pm 0.21$ & $1.51 \pm 0.18$ & $1.31 \pm 0.15$ & $0.96 \pm 0.11$ & $0.83 \pm 0.10$ & $0.37 \pm 0.04$ \\
Sig $\left(\mathbb{E}_{T}^{\mathrm{T}}\right)$ & $1.39 \pm 0.17$ & $1.21 \pm 0.14$ & $1.08 \pm 0.13$ & $0.81 \pm 0.09$ & $0.67 \pm 0.08$ & $0.31 \pm 0.04$ \\
$M_{T}$ & $1.23 \pm 0.15$ & $1.08 \pm 0.13$ & $1.00 \pm 0.12$ & $0.74 \pm 0.09$ & $0.61 \pm 0.07$ & $0.28 \pm 0.03$ \\
$p_{T}^{t r} \times \mathbb{E}_{T}^{t}$ & $1.16 \pm 0.14$ & $1.02 \pm 0.12$ & $0.96 \pm 0.11$ & $0.71 \pm 0.08$ & $0.58 \pm 0.07$ & $0.27 \pm 0.03$ \\
\hline
\end{tabular}

Table 5.8: Number of events after each selection cut for the chargino scan A5 - F5. 


\begin{tabular}{|c|c|c|c|c|c|c|}
\hline \multicolumn{7}{|c|}{$m_{\widetilde{\chi}_{1}^{ \pm}} \operatorname{scan}$ with $m_{\widetilde{\chi}_{2}^{0}}-m_{\tilde{\ell}}=-100 \mathrm{GeV}$} \\
\hline & A6 & B6 & C6 & D6 & E6 & F6 \\
\hline$\sigma \times B R$ & $0.06 \mathrm{pb}$ & $0.05 \mathrm{pb}$ & $0.04 \mathrm{pb}$ & $0.04 \mathrm{pb}$ & $0.03 \mathrm{pb}$ & $0.02 \mathrm{pb}$ \\
\hline$m_{\tilde{\chi}_{1}^{ \pm}}$ & $98 \mathrm{GeV}$ & $101 \mathrm{GeV}$ & $106 \mathrm{GeV}$ & $110 \mathrm{GeV}$ & $114 \mathrm{GeV}$ & $132 \mathrm{GeV}$ \\
\hline \multicolumn{7}{|l|}{ Cut } \\
\hline Presel & $1.69 \pm 0.19$ & $1.46 \pm 0.17$ & $1.19 \pm 0.14$ & $1.13 \pm 0.13$ & $0.97 \pm 0.11$ & $0.58 \pm 0.07$ \\
\hline$p_{T}^{\mu_{1}}$ & $1.67 \pm 0.19$ & $1.45 \pm 0.17$ & $1.19 \pm 0.14$ & $1.12 \pm 0.13$ & $0.97 \pm 0.11$ & $0.58 \pm 0.07$ \\
\hline$M_{\mu \mu}$ & $1.38 \pm 0.16$ & $1.16 \pm 0.13$ & $0.92 \pm 0.11$ & $0.83 \pm 0.09$ & $0.68 \pm 0.08$ & $0.34 \pm 0.04$ \\
\hline $\mathbb{E}_{T}$ & $0.99 \pm 0.11$ & $0.85 \pm 0.10$ & $0.69 \pm 0.08$ & $0.64 \pm 0.07$ & $0.54 \pm 0.06$ & $0.28 \pm 0.03$ \\
\hline$p_{T}^{t r}$ & $0.58 \pm 0.07$ & $0.50 \pm 0.06$ & $0.40 \pm 0.05$ & $0.38 \pm 0.04$ & $0.32 \pm 0.04$ & $0.17 \pm 0.02$ \\
\hline$M_{\mu_{1} t r}$ & $0.51 \pm 0.06$ & $0.44 \pm 0.05$ & $0.35 \pm 0.04$ & $0.33 \pm 0.04$ & $0.28 \pm 0.03$ & $0.14 \pm 0.02$ \\
\hline$\Sigma_{\mathrm{p}_{\mathrm{T}}} / \mathrm{p}_{\mathrm{T}}^{\mathrm{tr}}$ & $0.36 \pm 0.04$ & $0.31 \pm 0.04$ & $0.25 \pm 0.03$ & $0.24 \pm 0.03$ & $0.20 \pm 0.02$ & $0.10 \pm 0.01$ \\
\hline $\operatorname{Sig}\left(\mathbb{E}_{T}\right)$ & $0.30 \pm 0.04$ & $0.25 \pm 0.03$ & $0.20 \pm 0.02$ & $0.19 \pm 0.02$ & $0.16 \pm 0.02$ & $0.08 \pm 0.01$ \\
\hline$M_{T}$ & $0.27 \pm 0.03$ & $0.23 \pm 0.03$ & $0.18 \pm 0.02$ & $0.18 \pm 0.02$ & $0.15 \pm 0.02$ & $0.07 \pm 0.01$ \\
\hline$p_{T}^{t r} \times \mathbb{E}_{T}$ & $0.25 \pm 0.03$ & $0.22 \pm 0.03$ & $0.17 \pm 0.02$ & $0.17 \pm 0.02$ & $0.14 \pm 0.02$ & $0.07 \pm 0.01$ \\
\hline
\end{tabular}

Table 5.9: Number of events after each selection cut for the chargino scan A6 - F6.

In summary, after applying all cuts the expected events for the SUSY signal ranges from $1.91 \pm 0.24$ to $0.07 \pm 0.01$ events. As an example, the limit on the cross section as a function of the chargino mass will be discussed in section 6.1 for the chargino mass scan from table 5.4. The limit on the cross section as a function of the mass difference between the neutralino and slepton (slepton scan) is also shown for the SUSY points Cx and Dx with $\mathrm{x}=1,2,3,4,5,6$. The chargino mass for these SUSY points is beyond the LEP limit.

\subsection{Systematic Uncertainties}

There are different sources of systematic uncertainties which are summarized in the following for the Standard Model background and the SUSY signal.

Standard Model background All Monte Carlo backgrounds are normalized to the data using the process $Z \rightarrow \mu^{+} \mu^{-}$and the resulting scale factor is applied on all MC processes. Therefore the cross section for the $Z / \gamma^{*} \rightarrow \mu^{+} \mu^{-}$process was varied by $\pm 10 \%$. The relative error of the cross section as a function of mass was taken into account by keeping the cross section fixed at the $\mathrm{Z}$ resonance and by varying the cross section for the DrellYan process at other masses by their relative uncertainty (PDF uncertainty) (e.g for a mass of $20 \mathrm{GeV}$ the cross section was varied by $+2.5 \%(-2.9 \%)$ ) [45]. The difference for not taking into account the relative uncertainty was taken as a systematic error. Also the smearing factors $\mathrm{A}$ and $\mathrm{B}$ for the transverse momentum of the muon (Eq. 4.1) were varied by $\pm 10 \%$.

The $p_{T}$ spectra of the reconstructed $Z / \gamma^{*}$ is not properly described in the PYTHIA 6.2 MC. As described in [67] the $p_{T}$ of the $Z / \gamma^{*}$ processes can be weighted at generator level, 
to describe the data correctly. The difference between weighting and and not weighting the Monte Carlo was taken as a systematic error.

The systematic error from the smearing of the 3rd selected charged track in the event (like in Eq. 4.1) was taken into account by variation of the smearing factors A and B by $\pm 10 \%$.

The linear fits for the weighting factors $\mathrm{w}\left(p_{T}^{\mu_{1}}, p_{T}^{\mu_{2}}\right)$ for same-sign and opposite-sign muon events, were varied by extending the range of the fit. The difference was taken as a systematic error. The weighting factor for QCD events with muons with $p_{T}^{\mu_{1}}>13 \mathrm{GeV}$ $\left(p_{T}^{\mu_{2}}>8\right)$ for the leading- $p_{T}$ muon (next-to-leading- $p_{T}$ muon) was set from 0.4 to 0.3 and the difference taken as a systematic error. The total systematic error for the scale factor for QCD events from anti-isolated data for opposite-sign muon pairs is $s^{o s}=0.83 \pm 0.07$ \pm 0.24 and for same-sign events the systematic error is $s^{s s}=0.89 \pm 0.08 \pm 0.06$.

The statistical error on the reducing factors for the transverse mass and scaled missing transverse energy for the QCD background were taken as a systematic error. In addition a systematic error was added for the uncertainty of the method used to estimate the QCD background: the efficiency of the cuts for the same-sign QCD background (estimated from the anti-isolated sample) was compared to the efficiency of the cuts for the isolated same-sign muon sample. The relative error was taken as a systematic uncertainty.

SUSY signal A large contribution to the systematic error for the signal is due to the error on the cross section of the $Z \rightarrow \mu^{+} \mu^{-}$process and the MC smearing. The cross section for the $Z / \gamma^{*} \rightarrow \mu^{+} \mu^{-}$process was varied by $\pm 10 \%$. Also the smearing factors $\mathrm{A}$ and $\mathrm{B}$ for the transverse momentum of the muon (Eq. 4.1) were varied by $\pm 10 \%$. The systematic error from the smearing of the 3rd selected charged track in the event (like in Eq. 4.1) was taken into account by variation of the smearing factors $A$ and $B$ by $\pm 10 \%$. The systematic error resulting from the Drell-Yan PDF uncertainty and the correction of the $p_{T}$ spectra of the PYTHIA $Z / \gamma^{*}$ Monte Carlo has also been taken into account.

The individual relative systematic uncertainties after the last cut are shown in table 5.5 for background and SUSY point D3. The normalization of the QCD background has been determined from the difference between the data and expected Drell-Yan background. Therefore some of the systematic errors (e.g. PDF uncertainty, correction of the $p_{T}$ spectra of $Z / \gamma^{*}$ ), are strongly anti-correlated between Drell-Yan and QCD background, resulting in a smaller error for the sum of all backgrounds as for the individual components.

For the signal, the main systematic error comes from the uncertainty on the cross section for the $Z / \gamma^{*} \rightarrow \mu^{+} \mu^{-}$process of $\pm 10 \%$. 


\begin{tabular}{|c|c|c|c|c|c|}
\hline syst. uncertainty & $\begin{array}{c}\text { Sum } \\
{[\%]}\end{array}$ & $\begin{array}{c}Z / \gamma^{*} \rightarrow \mu^{+} \mu^{-} \\
{[\%]}\end{array}$ & $\begin{array}{c}Z / \gamma^{*} \rightarrow \tau^{+} \tau^{-} \\
{[\%]}\end{array}$ & $\begin{array}{c}\text { QCD } \\
{[\%]}\end{array}$ & $\begin{array}{c}\text { D3 } \\
{[\%]}\end{array}$ \\
\hline$\sigma\left(Z / \gamma^{*} \rightarrow \mu^{+} \mu^{-}\right)$ & 0.48 & 0.00 & 0.00 & 0.00 & 11.11 \\
$p_{T}^{\mu}$ smearing & 0.26 & 0.78 & 0.78 & - & 1.22 \\
3rd track smearing & 0.05 & 0.00 & 0.00 & - & 0.11 \\
PDF uncertainty & 0.74 & 2.32 & 2.31 & 0.08 & 0.18 \\
$p_{T}$ spectra correction & 0.79 & 10.82 & 10.22 & 6.28 & 0.02 \\
of $Z / \gamma^{*}$ & & & & & \\
\hline weight for high & & & & & \\
$p_{T}$ QCD muons & 5.49 & - & - & 1.63 & - \\
range linear fit & 1.63 & - & - & 24.26 & - \\
method QCD from data & 24.26 & - & - & 8.83 & - \\
reducing factors & 8.83 & - & 10.51 & 40.78 & 11.37 \\
\hline \hline sum & 26.47 & 11.09 & & &
\end{tabular}

Table 5.10: The individual relative systematic errors after the last cut, for the sum of all backgrounds, the Drell-Yan processes, the QCD background and the mSUGRA Point D3. 


\section{Chapter 6}

\section{Results}

A search for Supersymmetry has been performed in a data sample with an integrated luminosity of $\sim 300 \mathrm{pb}^{-1}$ collected with the D $\varnothing$ detector. No SUSY signal was observed and therefore limits on the production cross section and branching fraction $\sigma \times B R(3 \ell)$ in the final states $\widetilde{\chi}_{1}^{ \pm} \widetilde{\chi}_{2}^{0} \rightarrow 3 \ell+\mathbb{E}_{T}$ with any combination of three charged leptons, have been set. Two candidate events have been selected from the data sample and event displays for these events are shown. In section 6.3 of this chapter, the results of the combination with three additional decay channels and the obtained limit on the cross section is presented.

\subsection{Limit for $\sigma \times B R(3 \ell)$}

The limits were calculated at 95\% confidence level (CL) using the modified frequentist approach [66]. This method calculates the confidence level for searches for new particles, where the expected signal and background are small enough to require the use of the Poisson statistics. The confidence levels are computed by comparing the observed data configuration to the signal expectations for two hypotheses: the background hypothesis where only the Standard Model background processes contribute to the accepted event rate and the signal+background hypothesis where the SUSY signal adds to the background. The likelihood ratio of the Poisson probabilities of the two hypothesis is then defined as

$$
\mathrm{Q}=\frac{\mathrm{P}_{\text {Poisson }}(\text { data } \mid \text { signal }+ \text { background })}{\mathrm{P}_{\text {Poisson }}(\text { data } \mid \text { background })} .
$$

To test the consistency of the data with the signal+background hypothesis, the confidence level is defined as $\mathrm{CL}_{\mathrm{s}+\mathrm{b}}$

$$
1-\mathrm{CL}_{\mathrm{s}+\mathrm{b}}=\mathrm{P}\left(\mathrm{Q} \leq \mathrm{Q}_{\text {obs }} \mid \text { signal }+ \text { background }\right)
$$

as the fraction of experiments in a large ensemble of signal+background experiments which would produce results less signal like than the observed data. By definition a signal+background hypothesis is excluded at $95 \%$ confidence level if $\mathrm{CL}_{\mathrm{s}+\mathrm{b}}<0.05$ [68]. 
The calculated limits depend on the signal and background detection efficiency, the integrated luminosity and the number of candidates selected from the data events. The effects of the systematic uncertainties of the signal and background as well as the uncertainty of the integrated luminosity are incorporated. The number of background events is $1.75 \pm 0.36$ (stat.) \pm 0.46 (syst.) and 2 candidates were selected from the data sample. The number of expected signal events after all cuts is shown in table 5.4-5.9 for all SUSY points considered.

Table 6.1 summarizes the upper limits on $\sigma \times B R(3 \ell)$ from the analysis of the decay channel with at least two muons. For guidance, the masses of the chargino, neutralino and the slepton masses are shown. The theoretical cross section times branching fraction $\sigma \times B R(3 \ell)$ for the final states with any combination of three charged leptons is also shown. The observed limit $\sigma_{o b s}$ is the actual limit on the production cross section for associated chargino neutralino pair production.

Figure 6.1 shows the limit on $\sigma \times B R(3 \ell)$ as a function of the chargino mass. The signal efficiencies are taken from the SUSY points A3-F3, which represent the best signal detection efficiency. The expectations from the mSUGRA model, and also the scenario

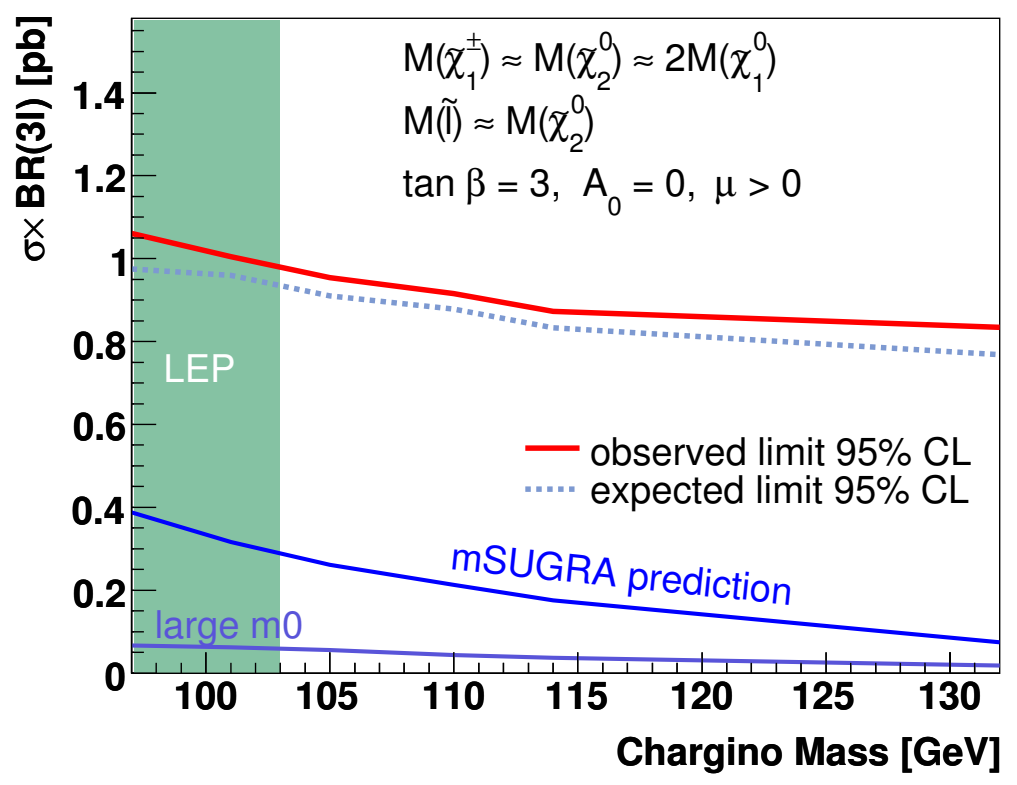

Figure 6.1: Limit on $\sigma \times B R(3 \ell)$ as a function of the chargino mass compared with the expectation of the mSUGRA model.

with large slepton masses labeled with 'large m0', is shown. For large slepton masses the $\widetilde{\chi}_{1}^{ \pm} \widetilde{\chi}_{2}^{0}$ decay only via virtual bosons.

From LEP direct searches a lower value than $103 \mathrm{GeV}$ for the chargino mass was excluded [21]. The upper limit on the cross section and branching fraction $\sigma \times B R(3 \ell)$ by the RunI searches is $1.5 \mathrm{pb}[69,70]$, when taking into account all the final states with any combination of charged leptons. 


\begin{tabular}{|c|c|c|c|c|c|c|}
\hline SUSY Point & $\begin{array}{c}m_{\widetilde{\chi}_{2}^{0}} \\
{[\mathrm{GeV}]}\end{array}$ & $\begin{array}{c}m_{\tilde{\chi}_{1}^{ \pm}} \\
{[\mathrm{GeV}]}\end{array}$ & $\begin{array}{c}m_{\tilde{\ell}} \\
{[\mathrm{GeV}]}\end{array}$ & $\begin{array}{c}\sigma \times B R(3 \ell) \\
{[\mathrm{pb}]}\end{array}$ & $\begin{array}{l}\sigma_{\text {exp }} \\
{[\mathrm{pb}]}\end{array}$ & $\begin{array}{c}\sigma_{o b s} \\
{[\mathrm{pb}]}\end{array}$ \\
\hline A1 & 102 & 98 & 92 & 1.03 & 1.91 & 2.00 \\
\hline A2 & 102 & 98 & 97 & 0.77 & 3.90 & 4.11 \\
\hline A3 & 102 & 98 & 102 & 0.39 & 0.98 & 1.06 \\
\hline A4 & 102 & 98 & 108 & 0.35 & 1.24 & 1.28 \\
\hline A6 & 102 & 103 & 208 & 0.06 & 1.12 & 1.17 \\
\hline B1 & 106 & 101 & 96 & 0.86 & & \\
\hline B2 & 106 & 101 & 101 & 0.66 & 3.84 & 4.05 \\
\hline B3 & 106 & 101 & 106 & 0.32 & 0.96 & 1.00 \\
\hline B4 & 106 & 101 & 112 & 0.28 & 1.17 & 1.20 \\
\hline B5 & 106 & 101 & 118 & 0.24 & 1.11 & 1.21 \\
\hline B6 & 106 & 107 & 209 & 0.05 & 1.05 & 1.14 \\
\hline $\mathrm{C} 1$ & 110 & 106 & 99 & 0.73 & 1.91 & 1.99 \\
\hline $\mathrm{C} 2$ & 110 & 106 & 105 & 0.55 & 4.00 & 4.26 \\
\hline $\mathrm{C} 3$ & 110 & 106 & 110 & 0.26 & 0.91 & 0.95 \\
\hline $\mathrm{C} 4$ & 110 & 106 & 116 & 0.23 & 1.21 & 1.26 \\
\hline $\mathrm{C} 5$ & 110 & 106 & 122 & 0.19 & 0.96 & 1.04 \\
\hline C6 & 110 & 111 & 210 & 0.04 & 1.13 & 1.18 \\
\hline$\overline{\mathrm{D} 1}$ & 114 & 110 & 103 & 0.60 & 1.74 & 1.79 \\
\hline D2 & 114 & 110 & 109 & 0.47 & 3.61 & 3.76 \\
\hline D3 & 114 & 110 & 114 & 0.21 & 0.88 & 0.92 \\
\hline D4 & 114 & 110 & 120 & 0.19 & 1.11 & 1.21 \\
\hline D5 & 114 & 110 & 126 & 0.15 & 1.08 & 1.13 \\
\hline D6 & 114 & 114 & 210 & 0.04 & 1.03 & 1.08 \\
\hline E1 & 118 & 114 & 107 & 0.51 & 1.72 & 1.77 \\
\hline E2 & 118 & 114 & 112 & 0.40 & 2.86 & 3.00 \\
\hline E3 & 118 & 114 & 118 & 0.18 & 0.83 & 0.87 \\
\hline E4 & 118 & 114 & 124 & 0.15 & 1.09 & 1.13 \\
\hline E5 & 118 & 114 & 130 & 0.13 & 1.08 & 1.13 \\
\hline E6 & 118 & 119 & 211 & 0.03 & 1.03 & 1.12 \\
\hline F1 & 135 & 132 & 124 & 0.26 & 1.47 & 1.53 \\
\hline F2 & 135 & 132 & 130 & 0.18 & 3.01 & 3.17 \\
\hline F3 & 135 & 132 & 136 & 0.07 & 0.77 & 0.83 \\
\hline $\mathrm{F} 4$ & 135 & 132 & 142 & 0.07 & 1.09 & 1.12 \\
\hline F5 & 135 & 132 & 148 & 0.06 & 1.03 & 1.08 \\
\hline F6 & 132 & 132 & 212 & 0.02 & 1.18 & 1.28 \\
\hline
\end{tabular}

Table 6.1: The limits on the cross section $\sigma \times B R(3 \ell)$ determined from events with at least two muons, an additional track and a large amount of $\mathbb{E}_{T}$. 
The decay channel investigated by this analysis alone is not significant for the mSUGRA predictions. Nevertheless, the observed limit calculated from the results of this decay channel $\widetilde{\chi}_{1}^{ \pm} \widetilde{\chi}_{2}^{0} \rightarrow \mu \mu \ell+\mathbb{E}_{T}$ alone, provides a stronger limit than the RunI data. The analysis improves the former results by excluding cross sections of $1.0 \mathrm{pb}$ for a chargino mass of $103 \mathrm{GeV}$ down to $0.85 \mathrm{pb}$ for a chargino mass of $132 \mathrm{GeV}$.

The slepton scans for SUSY points C1-C6 and D1-D6 are shown in figure 6.2. The
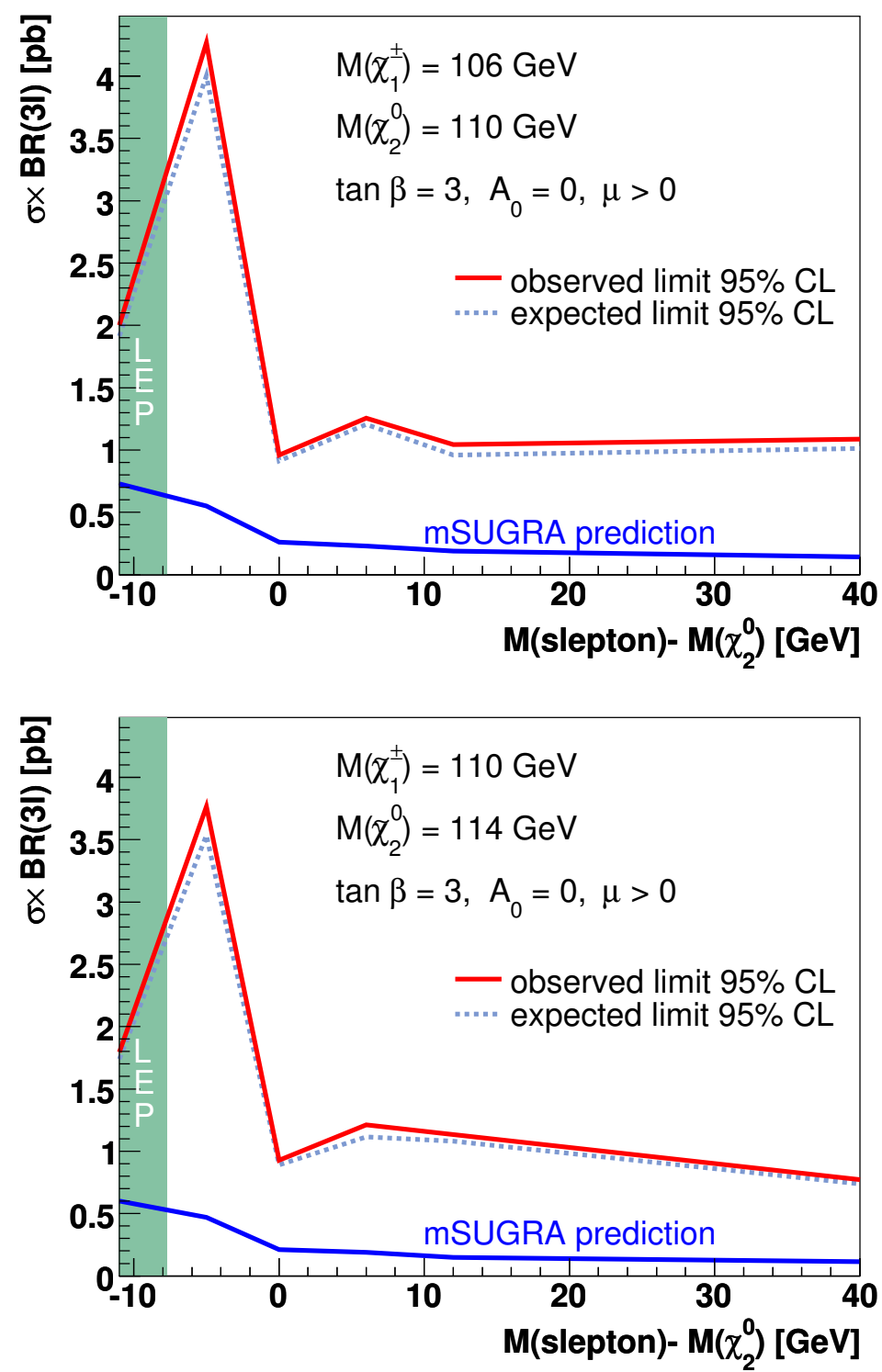

Figure 6.2: Limit on $\sigma \times B R(3 \ell)$ as a function of the mass difference between the slepton and the $\widetilde{\chi}_{2}^{0}$ compared with the expectation of the mSUGRA model.

chargino masses for these SUSY points are $106 \mathrm{GeV}(110 \mathrm{GeV})$ respectively, in a region not excluded by the LEP results. The observed limit excludes cross sections higher than 
$0.95 \mathrm{pb}(0.92 \mathrm{pb})$ for the SUSY points $\mathrm{Cx}(\mathrm{Dx})$, where the $\widetilde{\chi}_{1}^{ \pm} \widetilde{\chi}_{2}^{0}$ decay via virtual sleptons. For the region where $\widetilde{\chi}_{2}^{0}$ can decay via real sleptons one of the leptons from the neutralino decay can have a very low transverse momentum. The leptonic branching fraction drops at $m_{\tilde{\ell}} \lesssim m_{\widetilde{\chi}_{2}^{0}}$ because of the minimal phase space for two-body decays into real sleptons. The limits that can be set are quite large, but a more significant result can be obtained when additional decay channels are combined with the results from this analysis. For slepton masses larger than the neutralino mass the cross section limit is about $1 \mathrm{pb}$.

In summary, the reach of the presented analysis goes beyond that of Run I. An exclusion or discovery of a SUSY particle in the region of the mSUGRA parameter space investigated needs a combination with other decay channels. The results are presented in section 6.3.

\subsection{Event Displays of the Two Candidate Events}

The two data candidate events that passed all selection cuts are described in the following. The reconstructed physics objects in the event (muons, tracks, missing transverse energy and jets (if any)) are shown in dedicated event displays. The measured kinematic properties of the physics objects allow their identification in the event displays of the DØ detector.

The first candidate is likely to be an Drell-Yan event, whereas the second candidate contains a jet, and is likely to be a QCD event. Both events are shown with their run number, event number, date and time of recording. Three different views for each candidate are shown and discussed in detail.

The $\mathrm{D} \emptyset$ coordinate system is as discussed in section 3.2: the $\mathrm{z}$ axis is defined by the direction of the protons, the $\mathrm{x}$ axis points toward the center of the Tevatron ring and the $\mathrm{y}$ axis points vertically. The nominal collision point is the origin $(0,0,0)$ in these coordinates.

The first selected candidate event was recorded by the DØ detector in January 2004. It has the following characteristics:

$$
\begin{aligned}
\text { transverse momenta : } & p_{T}^{\mu_{1}}=26.17 \mathrm{GeV}, p_{T}^{\mu_{2}}=11.11 \mathrm{GeV}, p_{T}^{t r}=6.63 \mathrm{GeV}, \\
\text { pseudorapidity : } & \eta_{\mu_{1}}=-0.99, \eta_{\mu_{2}}=-1.09, \eta_{t r}=-1.03, \\
\text { azimuthal angle : } & \phi_{\mu_{1}}=0.35\left(20^{\circ}\right), \phi_{\mu_{2}}=2.45\left(140^{\circ}\right), \phi_{t r}=4.50\left(258^{\circ}\right), \\
\text { missing transverse energy : } & \mathbb{E}_{T}=31.94 \mathrm{GeV}, \\
\text { invariant dimuon mass : } & M_{\mu \mu}=29.63 \mathrm{GeV},
\end{aligned}
$$

Figure 6.3 shows an $y-z$ view of the inner tracker and calorimeter. The collision point of the event is slightly shifted into the $\mathrm{z}$ direction from the center of the detector. The primary vertex is reconstructed from the central tracks. Tracks deposit energy in the electromagnetic calorimeter (red) and the hadronic calorimeter (blue). The muons reach the muon system and are detected in its layers. These are marked as red, orange and green for the A, B and C layer respectively. By knowing the pseudorapidity of the particles the 
selected track in the event at $\eta=-1.09$ and $\phi=4.5\left(258^{\circ}\right)$ can be identified. The track is in the bottom part of the detector. Most probably it is an electron.

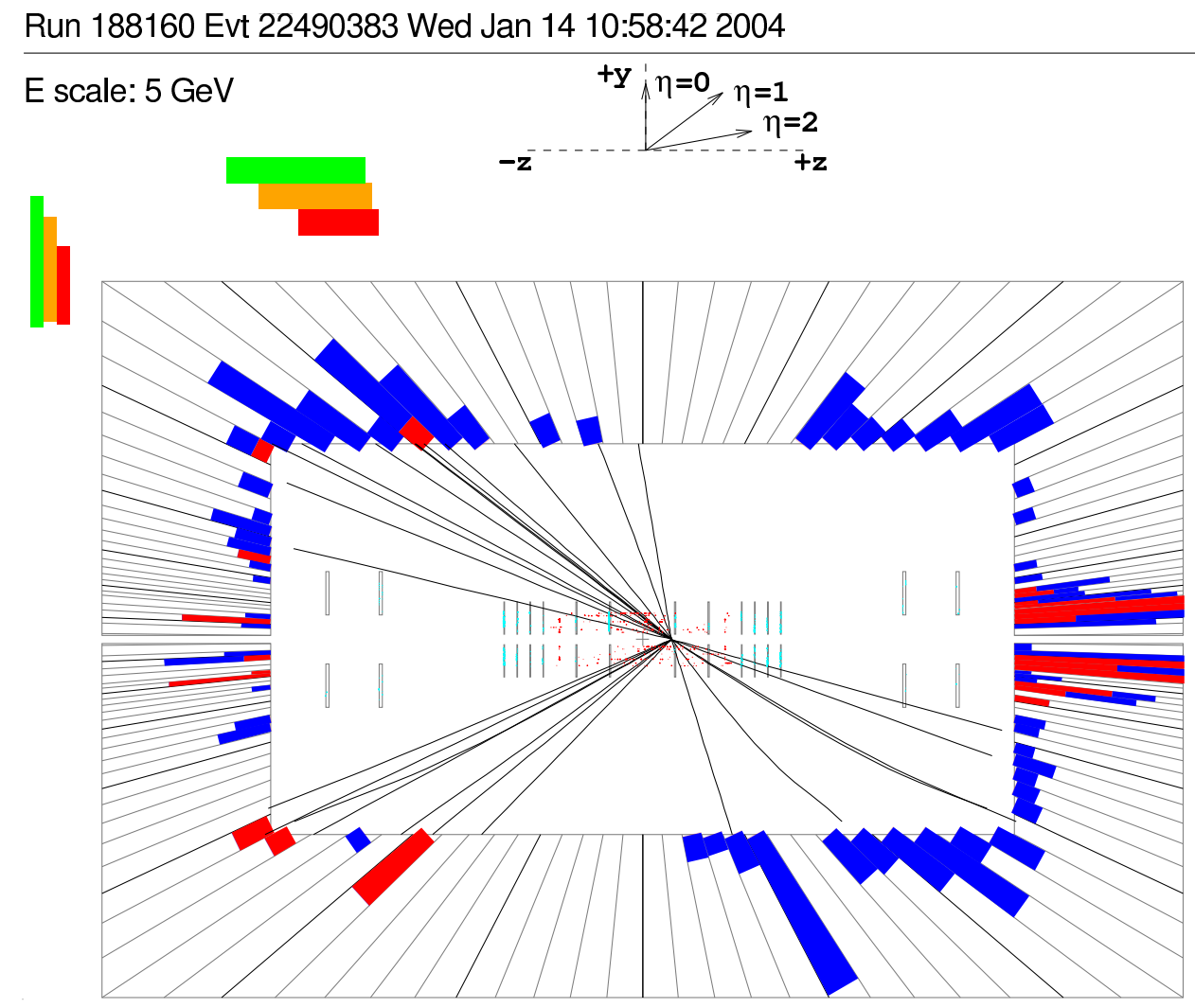

Figure 6.3: Two dimensional $y-z$ view of the first candidate event.

The selected muons are clearly visible from their associated layers in the muon system. The identification of the central track associated with the muon layers is visible in the $x-y$ view of the $D \varnothing$ detector in figure 6.4. It shows the inner tracker with the same central tracks as in figure 6.3 and the energy deposit in the calorimeter. The collision point is shown in the center surrounded by the most inner detector SMT with its cylindrical layers and the hits left by the particles marked in red. The CFT detector surrounds the SMT detector. Its cylindrical layers are shown with the hits marked in blue. The central tracks are reconstructed from the hits in both track detectors and are marked in black. The curvature corresponds to the $p_{T}$ of the track as both track detectors are located in a solenoid with a magnetic field.

The two central tracks associated with the muons in the event are visible at $\phi=0.35$ as a high- $p_{T}$ track with hits in the A-layer and $p_{T}^{\mu_{1}}=26.17 \mathrm{GeV}$. (The muon visible in the $\mathrm{B}$ and $\mathrm{C}$ layer belong to a low- $p_{T}$ muon.) The second muon can be identified by the central track at $\phi=2.45$ and the hits in all layers of the muon system. The cells of the calorimeter pointing to the beam pipe ( $\mathrm{z}$ axis) are shown with the amounts of energy left in the cells. The amount of missing transverse energy $\left(\mathbb{E}_{T}\right)$ in the event is shown in yellow. It does not 
correspond to the $\mathscr{E}_{T}$ used in the analysis, as all corrections for the jet energy scale and muon energy loss corrections are missing. The third track in the event is visible at $\phi=4.5$ with its amount of energy left in the electromagnetic calorimeter marked in red.

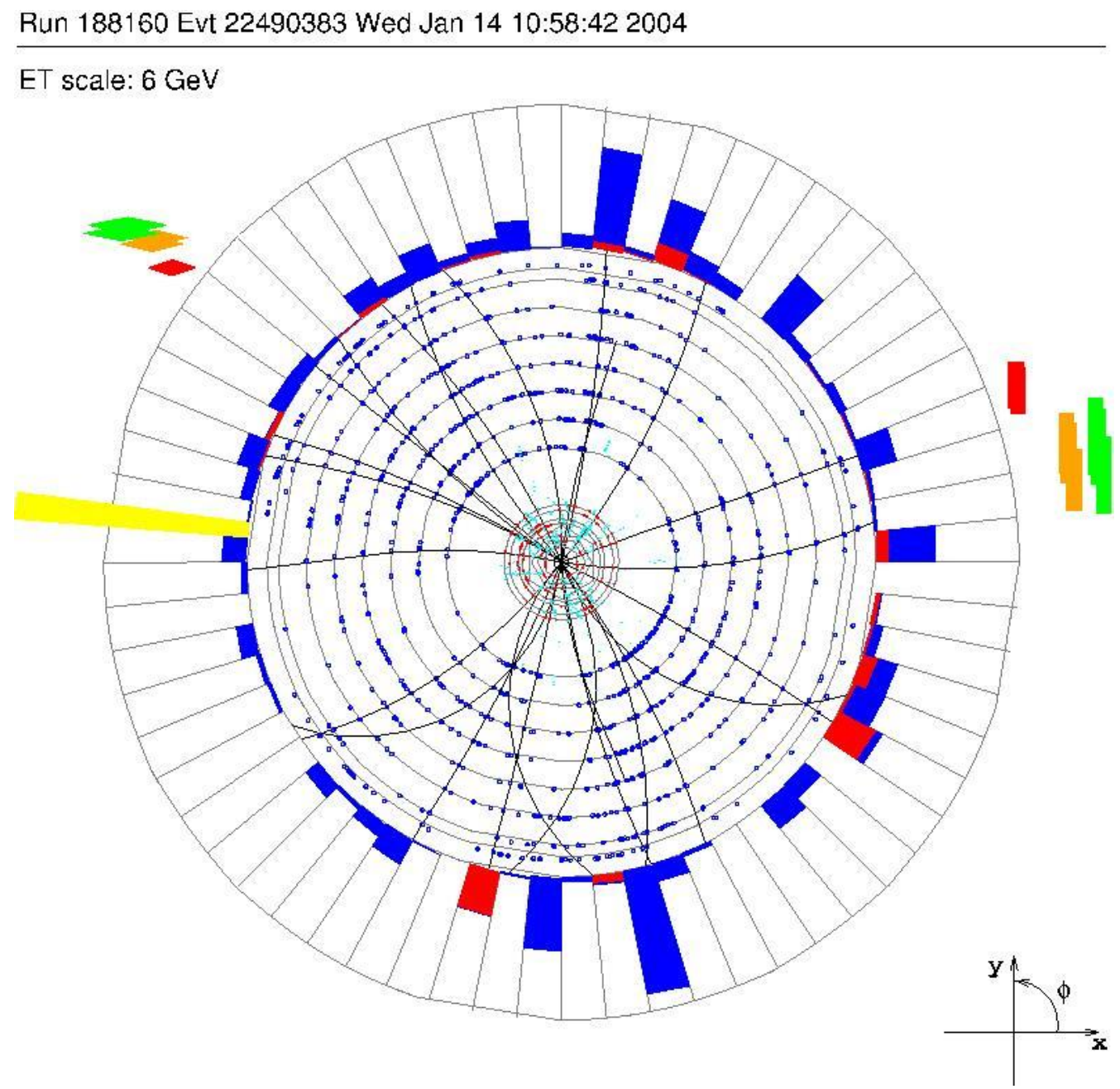

Figure 6.4: The $x-y$ view of the first candidate event.

A more detailed picture of the muon system is shown in figure 6.5. It is the same $x-y$ view as in figure 6.4. The inner tracker with the reconstructed central tracks and the calorimeter cells are drawn schematically.

The two muons are visible as reconstructed tracks in the layers of the muon system. The muon with $p_{T}^{\mu_{1}}$ is visible at $\phi=0.35$ (20 degrees). Its track is marked as blue in the A layer and the hits in red. The second selected muon with $p_{T}^{\mu_{2}}$ is visible at $\phi=2.45$ (140 degrees). Its track is marked in green in the B and C layer. This track is reconstructed from the tracks (marked in blue) and the so-called segments (red rectangular) of the hits the muon chambers in the A, B and C layers of the muon system. 


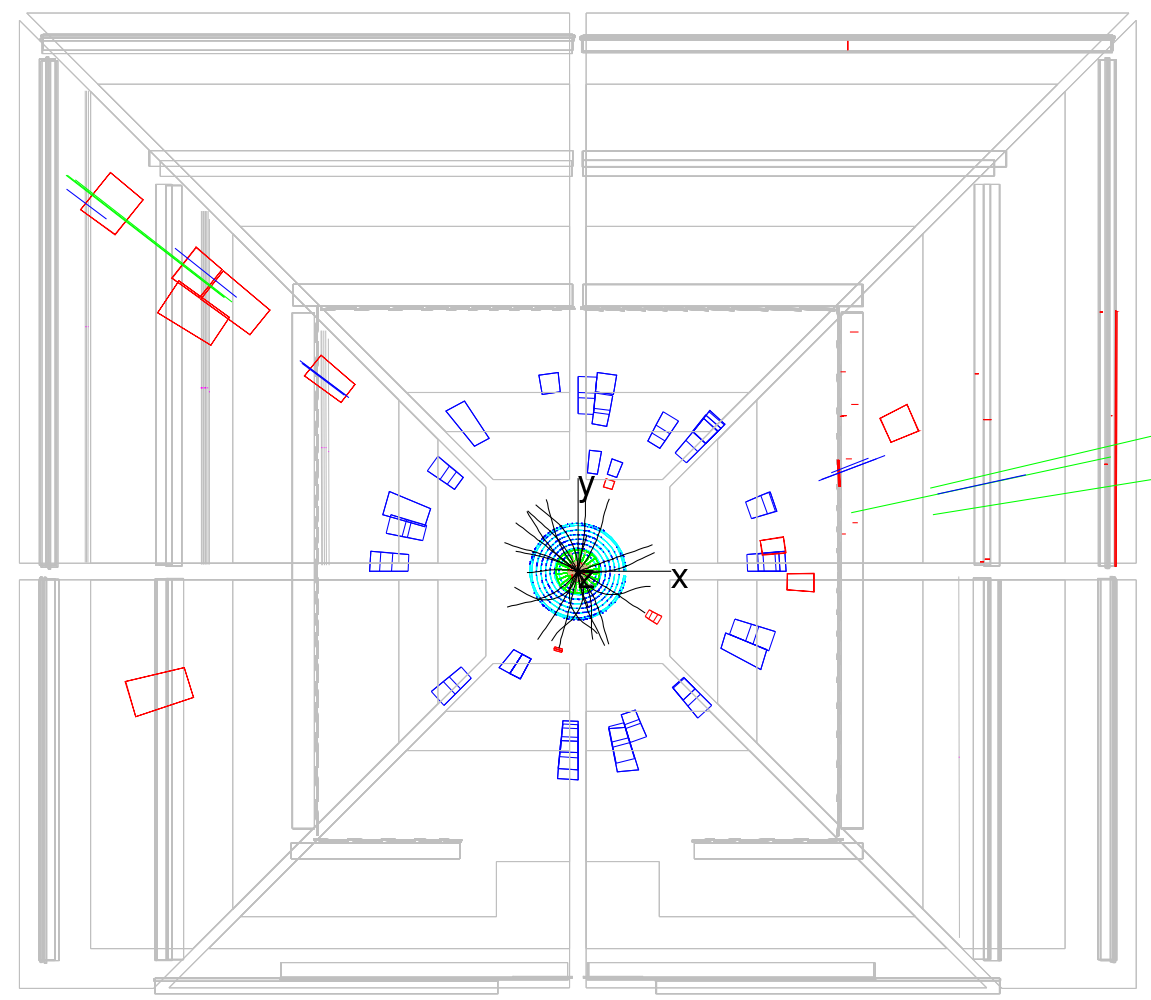

Figure 6.5: The $x-y$ view of the first candidate. 
The second candidate was recorded by the $\mathrm{D} \emptyset$ detector in an earlier data taking period in May 2003. It has the following characteristics:

$$
\begin{aligned}
\text { transverse momenta : } & p_{T}^{\mu_{1}}=23.61 \mathrm{GeV}, p_{T}^{\mu_{2}}=5.04 \mathrm{GeV}, p_{T}^{t r}=13.16 \mathrm{GeV}, \\
\text { pseudorapidity : } & \eta_{\mu_{1}}=-1.03, \eta_{\mu_{2}}=0.78, \eta_{t r}=-0.08, \\
\text { azimuthal angle : } & \phi_{\mu_{1}}=3.56\left(203^{\circ}\right), \phi_{\mu_{2}}=2.52\left(144^{\circ}\right), \phi_{t r}=4.52\left(259^{\circ}\right), \\
\text { missing transverse energy : } & E_{T}=39.90 \mathrm{GeV}, \\
\text { invariant dimuon mass : } & M_{\mu_{\mu}}=25.00 \mathrm{GeV} \\
\text { invariant masses : } & M_{\mu_{1} t r}=23.82 \mathrm{GeV} M_{\mu_{2} t r}=15.50 \mathrm{GeV} \\
\text { jet energy : } & \mathrm{p}_{\mathrm{T}}^{\text {jet }}=25.06 \mathrm{GeV}
\end{aligned}
$$

Figure 6.6 shows the $\mathrm{y}-\mathrm{z}$ view of the inner tracker and calorimeter. This event is characterized by two collisions: the primary vertex of the event is reconstructed close to the nominal collision point. Tracks from the second collision are not considered during the analysis.

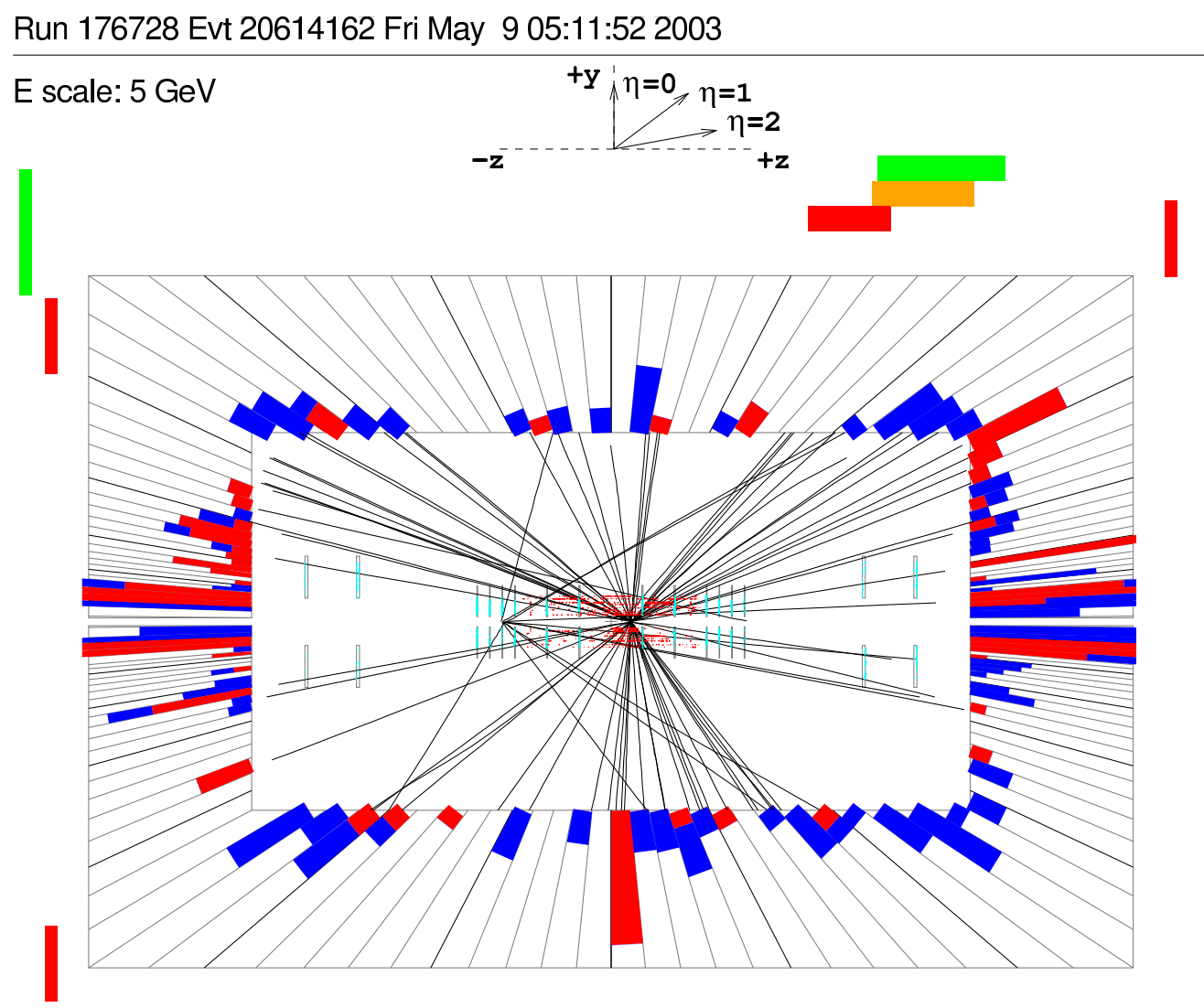

Figure 6.6: The $y-z$ view of the second candidate (including a jet).

The amount of tracks in the event and the energy left in the cells of the electromagnetic (red) and hadronic (blue) calorimeter indicate a two jet event. The reconstructed muons at 
$\eta=-1.03$ and $0.78\left(\phi=3.56\left(203^{\circ}\right)\right.$ and $\left.\phi=2.52\left(144^{\circ}\right)\right)$ were isolated and most probably belong to these jets.

A better identification of the muons and the selected track is possible in the $x-y$ view of the inner tracker and the calorimeter (figure 6.7). The cylindrical layers of the inner

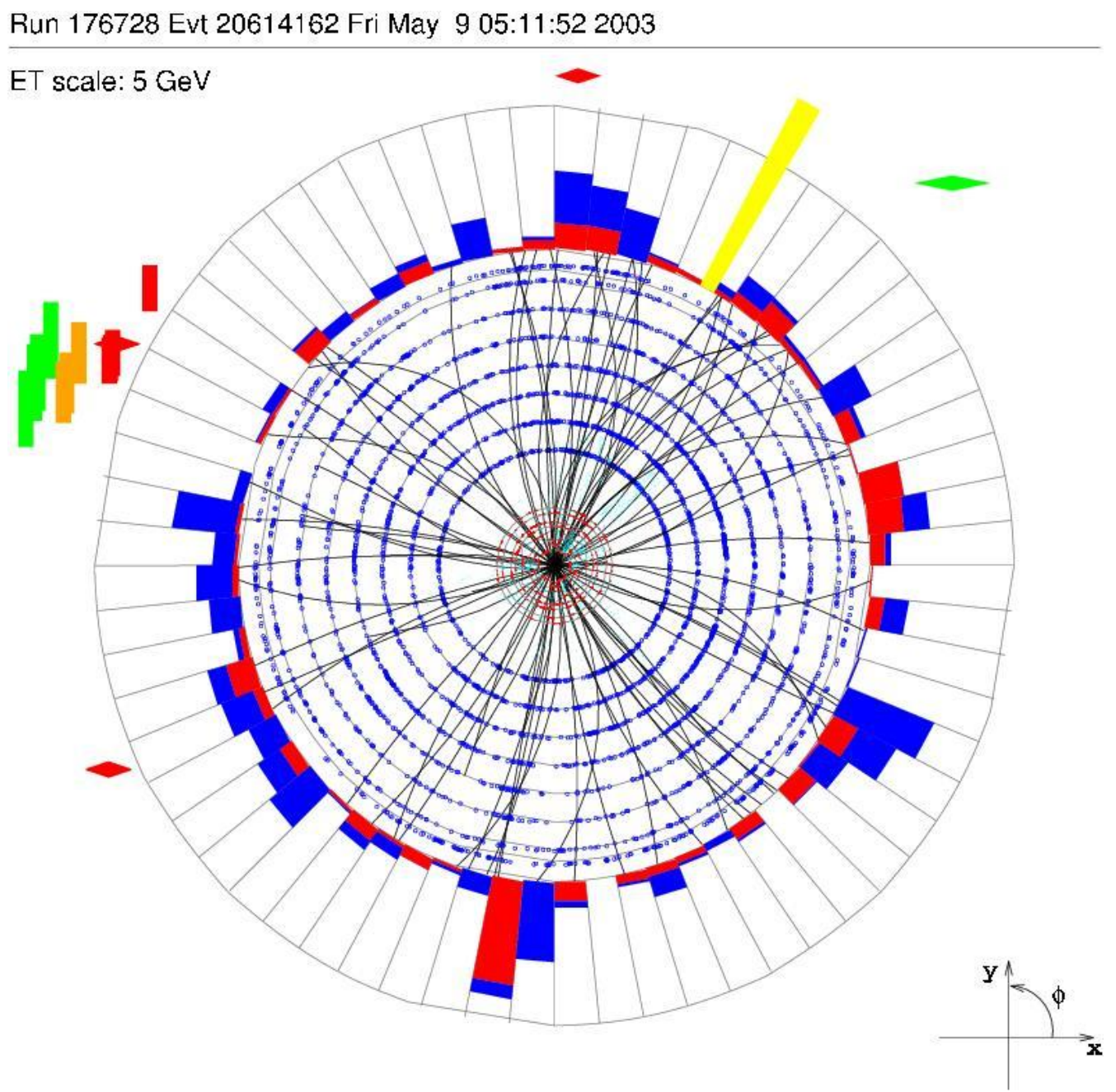

Figure 6.7: The x-y view of the second candidate (including a jet).

tracking detectors SMT and CFT are shown with the hits marked in red and blue respectively. The central tracks marked in black are reconstructed from these hits. The missing transverse energy (marked in yellow) must be corrected for the jet energy scale and muon energy loss in the calorimeter.

The isolated leading- $p_{T}$ muon in the event with $p_{T}^{\mu_{1}}=23.61 \mathrm{GeV}$ can be associated with the hits in the A layer and the central track at $\phi=3.56\left(203^{\circ}\right)$. The second muon with $p_{T}^{\mu_{2}}$ is visible at $\phi=2.52\left(144^{\circ}\right)$ with the hits in the A layer. The selected track in the event has $\phi=4.52\left(259^{\circ}\right)$.

The tracks in the muon system left by the muons in the event are shown in figure 
6.8 in a $x-y$ view of the $D \emptyset$ detector. This event display allows a detailed view of the reconstructed muons in the event. The inner detectors with the reconstructed central tracks and the energy deposit in the calorimeter are drawn schematically.

Run 176728 Evt 20614162 Fri May 9 05:11:52 2003

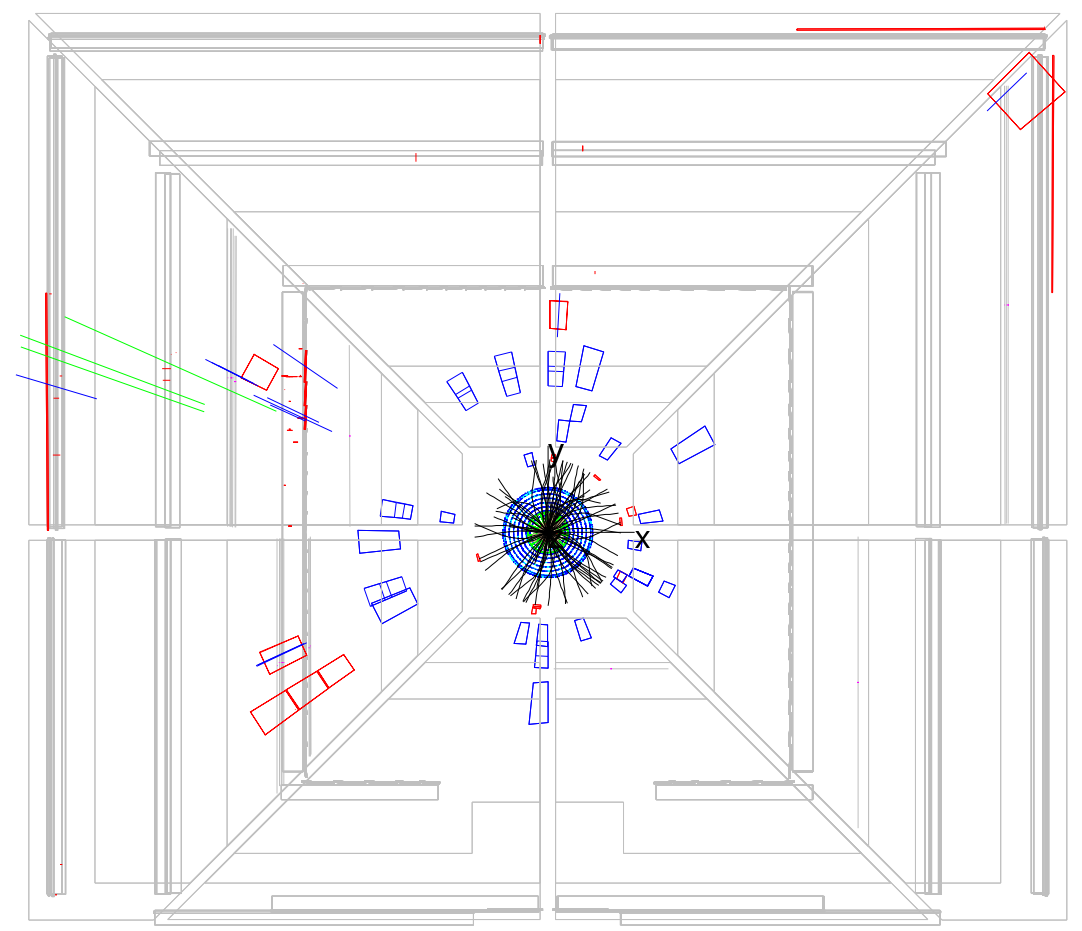

Figure 6.8: The $x-y$ view of the second candidate (including a jet).

The selected high- $p_{T}$ muon is visible as a blue track and a muon segment reconstructed from the hits in the muon chambers of the A layer at $\phi=3.56\left(203^{\circ}\right)$. The second muon is visible at $\left.\phi=2.52\left(144^{\circ}\right)\right)$ as a blue track and a red segment in the A layer of the muon system. The three green tracks around $\phi=2.7$ belong to muons from the jet of $p_{T}^{\text {jet }}$ $=25.06 \mathrm{GeV}$ selected in the event. 


\subsection{Combined Limit for $\sigma \times B R(3 \ell)$}

The leptons arising in the final state of the $\widetilde{\chi}_{1}^{ \pm} \widetilde{\chi}_{2}^{0}$ decay, can be of $e, \mu$ or $\tau$ flavor. Depending on the lepton content in the final state, three additional selection channels are defined [71, 72]: two electrons plus lepton (eel), two muons of the same sign $\left(\mu^{ \pm} \mu^{ \pm}\right)$, one electron, one muon plus lepton $(e \mu \ell)$. These selection channels are combined with the selection channel investigated in this dissertation: two muons plus lepton $(\mu \mu \ell)$.

The combined analysis ${ }^{1}$ is based on a data sample recorded by D $\varnothing$ detector between March 2002 and July 2004. The data sample corresponds to an integrated luminosity of $320 \mathrm{pb}^{-1}$. Events containing muons or electrons were selected for offline analysis by a three level trigger system (as described in section 3.2.4). A set of single and dilepton triggers was used to select the events, based on their characteristic energy deposit in the calorimeter, the presence of tracks in the tracking system and hits in the muon system.

The Standard Model backgrounds are similar to the backgrounds of the $\mu \mu \ell$ selection channel: Drell-Yan production, di-boson processes and multi-jet production, which is estimated from data. As in the case of the $\mu \mu \ell$ selection channel, the multi-jet background was determined from a data sample which is identical to the search sample, except for a reversed isolation requirement for the selected leptons.

Isolated electrons were defined by their energy deposit in the calorimeter and a track pointing to it. The momentum of the track and the energy deposit in the calorimeter were required to be consistent with the same electron energy. The isolated muons were defined as for the $\mu \mu \ell$ selection channel. The electron reconstruction efficiencies were measured using the $\mathrm{Z}$ boson decay, similar to the muon reconstruction efficiencies (see section 4.5.1).

Each of these selection channels require two identified leptons with a minimum transverse momentum and a large amount of missing transverse energy in the final state. The kinematic and topological characteristics of the events were used to discriminate the SUSY signal events from the background events. The number of observed data events is in good agreement with the expected Standard Model background events at all stages of the selection [72].

An event selection has been performed for each selection channel. The selection criteria were optimized to get the best average limit assuming that no signal would be observed. The obtained limits were calculated at 95\% CL using the modified frequentist approach [66]. The optimization of the cuts was based on SUSY signals inspired by mSUGRA. The mass relation $2 m_{\widetilde{\chi}_{1}^{0}} \cong m_{\widetilde{\chi}^{ \pm}} \cong m_{\widetilde{\chi}_{2}^{0}}$ was assumed and slepton masses $m_{\tilde{\ell}}$ were degenerate (no slepton mixing). The gauginos and slepton masses were varied in the mass range of $110 \mathrm{GeV}-130 \mathrm{GeV}$. This mass range is particularly interesting for a Supersymmetry search in the trilepton decay channel $q \bar{q} \rightarrow \widetilde{\chi}_{1}^{ \pm} \widetilde{\chi}_{2}^{0} \rightarrow 3 \ell+2 \widetilde{\chi}_{1}^{0}+v$ as the production cross section and leptonic branching fraction via slepton exchange is large.

After the application of a dedicated event selection and the combination of the four

\footnotetext{
${ }^{1}$ The results of the combined analysis are to be published and details presented here can also be found in [72].
} 
selection channels, three events were selected from the data sample and a total background of $2.93 \pm 0.54$ (stat.) \pm 0.57 (syst.) events is expected. The total background after all cuts is dominated by the multi-jet background (66\% and 53\% for the $\mu \mu \ell$ and $\mu^{ \pm} \mu^{ \pm}$selections, respectively) and di-boson backgrounds ( $80 \%$ and $88 \%$ for $e e \ell$ and $e \mu \ell$ respectively). The systematic uncertainty is introduced by numerous sources from which the dominating contribution is the modeling of the multi-jet background (4-40\%).

No evidence for Supersymmetry via associated chargino neutralino production was found. An upper limit on the production cross section and leptonic branching fraction $\sigma \times B R(3 \ell)$ was calculated for the combined selection channels. The results from the four selection channels were combined using the modified frequentist approach and taking into account correlated errors. A small fraction of signal events that was selected by more than one selection channel, was assigned to the selection with the largest signal-tobackground ratio and removed from all the other selection channels. For the two selection channels with two muons in the final state the overlap of QCD events was calculated to be $0.09 \pm 0.05$ (stat.) \pm 0.03 (syst.) by applying the additional selection cuts of the $\mu^{ \pm} \mu^{ \pm}$ selection channel on to the $\mu \mu \ell$ selection channel. The overlap is small, but was taken into account in the process of combination.

Figure 6.9 shows the expected (red dashed line) and observed limit (red solid line) as a function of the chargino mass $m_{\widetilde{\chi}_{1}^{ \pm}}$. The values of the SUSY parameters are indi-

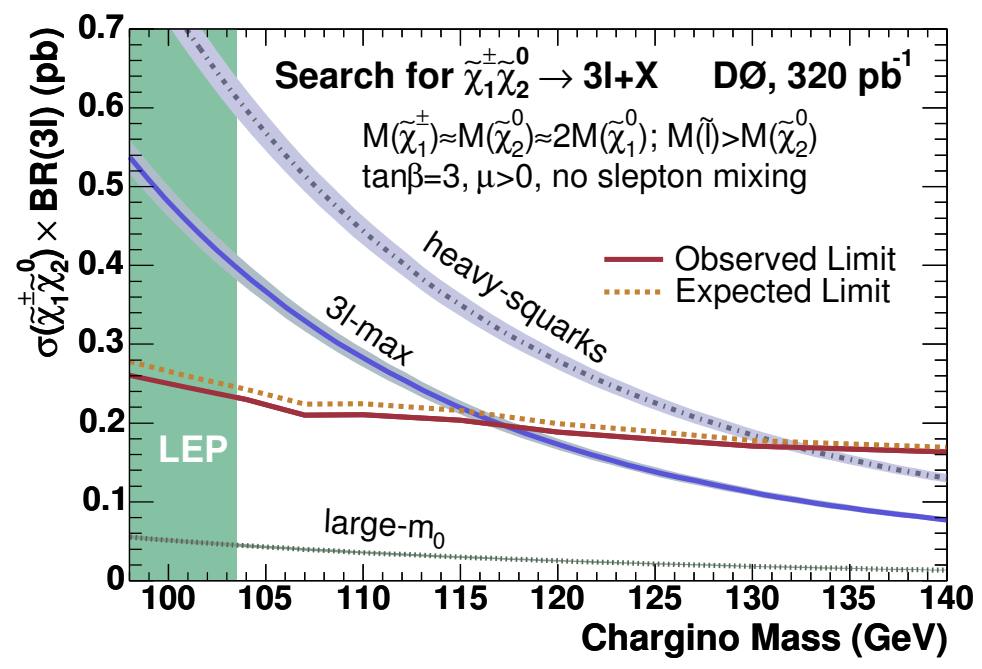

Figure 6.9: Limit on $\sigma \times B R(3 \ell)$ as a function of the chargino mass compared with the expectation for various SUSY scenarios. PDF and renormalization/factorization scale uncertainties are shown as shaded bands [72].

cated, the chargino and slepton mass differ by $\sim 1 \mathrm{GeV}$. The leptonic branching fraction of chargino and neutralino depends on the relative contribution from the slepton exchange graphs and the $W^{ \pm} Z$ exchange graphs. The contribution varies as a function of the slepton masses (see also section 2.5.4). For large slepton masses, the $W^{ \pm} Z$ exchange is dominant, with relatively small leptonic branching fractions (large- $\mathrm{m}_{0}$ scenario). The leptonic 
branching fraction for the three-body decays via virtual sleptons is maximally enhanced for $m_{\tilde{\ell}} \gtrsim m_{\widetilde{\chi}_{2}^{0}}$ (31-max scenario). The $\widetilde{\chi}_{1}^{ \pm} \widetilde{\chi}_{2}^{0}$ production cross section depends also on the squark masses due to the negative interference with the t-channel squark exchange (see section 2.5.4). Relaxing the scalar mass unification, the cross section is maximal in the limit of large squark masses (heavy-squarks scenario). A lower mass limit for the chargino mass of $m_{\tilde{\chi}_{1}^{ \pm}}>117 \mathrm{GeV}(132 \mathrm{GeV})$ at $95 \% \mathrm{CL}$ is set in the 31-max (heavy-squarks) scenario in a parameter space with enhanced leptonic branching fractions.

The leptonic branching fraction can be dominant if sleptons are light enough that twobody decays are possible. If the mass difference between neutralino and slepton is small, one of the leptons from the neutralino decay can have a very low transverse momentum. In this region of the parameter space, only the $\mu^{ \pm} \mu^{ \pm}$selection channel remains efficient. The analysis of this selection channel has no explicit requirement for an additional track (or lepton) in the event. Figure 6.10 shows the expected and observed limits for the mass difference between the slepton and the next-to-lightest neutralino. The leptonic branching

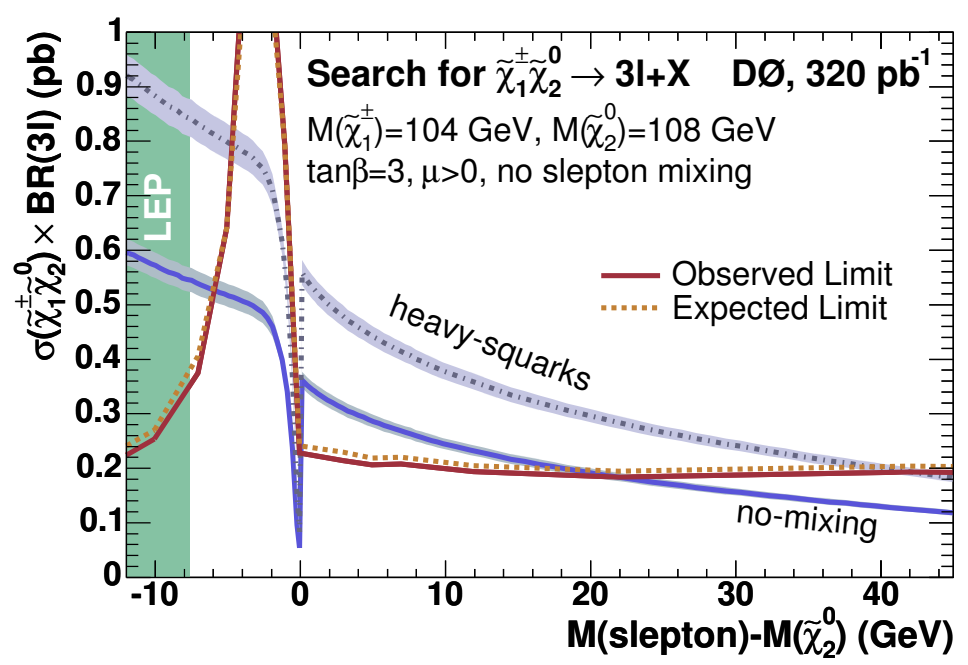

Figure 6.10: Limit on $\sigma \times B R(3 \ell)$ as a function of the mass difference between the slepton and the $\widetilde{\chi}_{2}^{0}$ compared with the expectation for the MSSM (no-mixing) and the heavysquarks scenario. PDF and renormalization/factorization scale uncertainties are shown as shaded bands. [72].

fraction drops sharply at $m_{\tilde{\ell}} \approx m_{\tilde{\chi}_{2}^{0}}$ because of the minimal phase space for two-body decays into real sleptons. A higher limit for $-6 \lesssim m_{\tilde{\ell}}-m_{\widetilde{\chi}_{2}^{0}}<0$ is set. The relevant SUSY parameters are indicated, the slepton mass ranges from $95 \mathrm{GeV}$ to $150 \mathrm{GeV}$.

The results obtained for the chargino mass scan improve significantly the upper limit of approximately $1.5 \mathrm{pb}$ set by the $\mathrm{D} \varnothing \mathrm{RunI}$ analysis $[69,70]$. The limit on $\sigma \times B R(3 \ell)$, $m_{\widetilde{\chi}_{1}^{ \pm}}$and $m_{\tilde{\ell}}$ is valid for more general Supersymmetry scenarios, as long as the mSUGRA inspired mass relation between the gauginos is valid and R-parity is conserved. 


\section{Chapter 7}

\section{Summary and Conclusions}

Supersymmetry or fermion boson symmetry, if it exists would answer some of the questions left open by the Standard Model. Supersymmetry must be broken, as no supersymmetric particles with the same masses as their Standard Model partners have been observed. There are several models of Supersymmetry, which are classified according to the mechanism of Supersymmetry breaking. In this dissertation the Minimal Supergravity Model (mSUGRA) serves as a paradigm. Only five parameters at a universal high energy scale are needed to describe the properties of the particles at the electroweak energy scale.

A search for the trilepton signal of chargino neutralino associated production in proton anti-proton collisions at a center-of-mass energy of $1.96 \mathrm{TeV}$ has been performed. The decay channel $q \bar{q} \rightarrow \widetilde{\chi}_{1}^{ \pm} \widetilde{\chi}_{2}^{0} \rightarrow \ell^{ \pm} v \widetilde{\chi}_{1}^{0} \mu^{ \pm} \mu^{\mp} \widetilde{\chi}_{1}^{0}$ under investigation contains two muons, an additional charged lepton and a large amount of missing transverse energy in the final state. The charged leptons arise from the subsequent decay of the chargino $\widetilde{\chi}_{1}^{ \pm}$and the neutralino $\widetilde{\chi}_{2}^{0}$, where the identification of the additional charged lepton is replaced with the requirement of an isolated track. The missing transverse energy is due to the neutrino and the $\widetilde{\chi}_{1}^{0}$ (the lightest supersymmetric particle) which are stable and escape detection.

Events with two isolated muons and an additional isolated track have been analyzed in a data sample collected from August 2002 to June 2004 by the DØ detector at the Fermilab Tevatron. The corresponding integrated luminosity is $\mathcal{L} \sim 300 \mathrm{pb}^{-1}$.

The Standard Model backgrounds for the trilepton signature arise from Drell-Yan, di-boson and multi-jet processes. Monte Carlo simulation has been used to study the properties of the Drell-Yan and di-boson processes. The background from multi-jet production has been determined from data. For this, a sample which is identical to the search sample except for a reversed muon isolation requirement has been analyzed.

A dedicated event selection has been applied to the data sample, as well as to the Monte Carlo simulated samples for the supersymmetric signal and the Standard Model backgrounds. The selection criteria have been optimized to obtain the best expected limit assuming that no signal is present. After all selection cuts, the expected number of background events is $1.75 \pm 0.36$ (stat.) \pm 0.46 (syst.), and 2 events have been selected from the data sample. The dominant background source is multi-jet production. The errors quoted are dominated by the statistical error of the multi-jet background and by the sys- 
tematic error of its modeling, respectively.

No evidence for Supersymmetry has been observed in the search for associated $\widetilde{\chi}_{1}^{ \pm} \widetilde{\chi}_{2}^{0}$ production in trilepton events. Upper limits on the production cross section and branching fraction $(\sigma \times B R(3 \ell))$ have been set from the analysis of the decay channel with two isolated muons and an additional isolated track.

In the mSUGRA model, the upper limits for $\sigma \times B R(3 \ell)$ range from $1.0 \mathrm{pb}$ for an assumed chargino mass of $103 \mathrm{GeV}$ down to $0.85 \mathrm{pb}$ for a chargino mass of $132 \mathrm{GeV}$. These limits have been calculated at $95 \%$ confidence level (CL) using the modified frequentist approach [66]. The limits from this analysis alone improve the RunI results of $1.5 \mathrm{pb}$ for similar chargino masses $[69,70]$.

A combination with three other decay channels has been performed. The results of the combined analysis have been submitted to Physical Review Letters for publication [72]. Here, the optimization of the selection cuts is based on supersymmetric trilepton signatures inspired by mSUGRA. Degenerate slepton masses (no slepton mixing) and the mass relation $2 m_{\widetilde{\chi}_{1}^{0}} \cong m_{\widetilde{\chi}_{1}^{ \pm}} \cong m_{\widetilde{\chi}_{2}^{0}}$ have been assumed. After all selection cuts, a total number of expected background events is $2.93 \pm 0.54$ (stat.) \pm 0.57 (syst.). The total background is dominated by the multi-jet and di-boson backgrounds. The combined analysis also shows no evidence for Supersymmetry via associated $\widetilde{\chi}_{1}^{ \pm} \widetilde{\chi}_{2}^{0}$ production. Instead of an upper limit on the cross section, a lower limit for the chargino mass of $m_{\widetilde{\chi}_{1}^{ \pm}}>117 \mathrm{GeV}$ at $95 \% \mathrm{CL}$ is set in a region of parameter space with enhanced leptonic branching fractions. The limit on the chargino mass is valid for more general Supersymmetry scenarios, as long as the mSUGRA inspired mass relation between the gauginos is valid and R-parity is conserved. This improves the former results from RunI searches and the results from direct searches for charginos at LEP $\left(m_{\widetilde{\chi}_{1}^{ \pm}}>103 \mathrm{GeV}\right)[21]$. 


\section{Appendix A}

\section{The Compressed Data Format: ThumbNail}

For an experiment in particle physics, the question of data storage is an essential one and has to be considered in view of the necessity to store all information and to easily access the reconstructed particle information for both data and MC. At DØ, the compressed data format ThumbNail fulfills the requirements of data size and data access. It is the official data format for analyses [73, 74]. As part of the presented thesis, the relevant software package concerning the data format for the muon particles has been designed.

\section{ThumbNail Definition}

The ThumbNail data format has been created in order to face the huge amount of data, collected by the DØ detector, to be stored for Run II. Events are written to tape at a frequency of $\sim 50 \mathrm{~Hz}$. Given the number of $\sim 500$ million events per year and a size of $\sim 200 \mathrm{~KB}$ per event, the disk space needed is $\sim 75000 \mathrm{~GB}$ per year for a non-compressed format [75]. In the ThumbNail format all relevant information is kept in the most compact way and redundant information is not longer stored. The compressing process starts with the different physics objects reconstructed by the reconstruction program DØReco. The physics objects information is compressed into one object called ThumbNailChunk. This compression is more powerful than the usual "gzip" algorithm, and care is taken that it preserves the necessary precision of the data to be stored.

The ThumbNailChunk has a size of $\sim 25 \mathrm{kB}$ per event, meeting the size requirements of $\mathrm{D} \emptyset$. The reading speed of the ThumbNail is currently of the order of 10 events/second. It will be possible to increase the reading speed to 30-50 events/second by improving the ThumbNail decompressing. In addition, the compressed data format also assures a fast access to the information needed for physics analysis and a fast transfer between different analyses sites is possible.

Figure A.1 shows the initial proposal for the space occupation of the physics objects in a ThumbNail file [74]. Muons use $2 \%$ of the space of one event. In the meantime more information of the calorimeter cells has been added doubling the ThumbNail size needed 


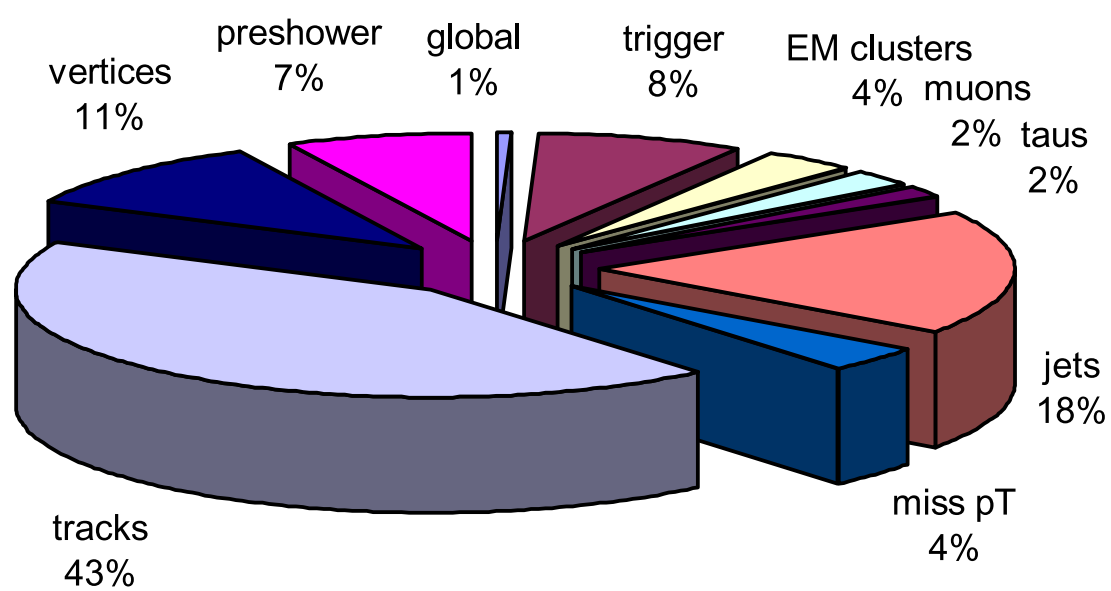

Figure A.1: Initial proposal for the space consumption of the physics objects, trigger information and history information (labeled as global) in a ThumbNail file.

to store one event.

\section{The information stored in the ThumbNail}

All relevant information for most physics analyses is stored in the ThumbNail. A typical ThumbNail file contains the following chunks:

- the ThumbNailChunk contains the reconstructed objects in compressed form: electromagnetic calorimeter clusters (all electrons, all isolated photons, cells), muons, taus, jets, missing transverse energy, tracks (all tracks found by the reconstruction, more detailed information for isolated tracks), vertices, preshower clusters (associated with electrons, taus and isolated tracks) and links to relate the physics objects to each other

- the HistoryChunk, providing information like run number, event number, the version number of the reconstruction software program

- the TriggerChunk, which provides information about the triggers and a list of triggers that fired

- the MCKineChunk is created for simulated events, and contains specific information for generated events

\section{ThumbNail Decompressing}

Information in the ThumbNail files, which is used for physics analysis, needs to be decompressed, before processed further for analysis. Saving and restoring packed fields is likely to be much slower than ones that have not been packed. 


\section{Bibliography}

[1] J. Wess and J. Bagger. Supersymmetry and Supergravity. Princeton Univ. Press, 1983.

[2] C.H. Lai (Ed.). Gauge Theory of Weak and Electromagnetic Interactions. World Scientific, 1981.

[3] G. Marciano, H. Pagels. Phys. Rep., 36C:137, 1978.

[4] D. Griffiths. Einführung in die Elementarteilchenphysik. Akad. Verl., Berlin, 1996.

[5] S. Eidelman et.al. The Review of Particle Physics. Phys.Lett.B, 592:1, 2004.

[6] G.G. Ross. Grand Unified Theories. Benjamin \& Cummings, 1985.

[7] Stephen P. Martin. A Supersymmetry Primer. In G.L.Kane, editor, Perspectives on Supersymmetry, pages 1-98. World Scientific Publishing, 1998.

[8] Y.A.Golfand and E.P.Likthman. JETP Letters, 13:452, 1971.

[9] V.P.Akulov. JETP Letters,16:621, 1972.

[10] J.Wess and B.Zumino. Phys.Lett.,B49:52, 1974.

[11] D.I.Kazakov. Beyond the Standard Model, volume BLTP, JINR, Dubna and ITEP, Moscow. Lectures given at European School of High Energy Physics, Aug.-Sept. 2000, Caramulo, Portugal, 2001.

[12] H.E.Haber. Introductory Low-Energy Supersymmetry. Lectures given at TASI 1992, 1993. hep-ph/9306207.

[13] W.deBoer. Grand Unified Theories and Supersymmetry in Particle Physics and Cosmology. Progr. in Nucl. and Particle Phys., 33:201, 1994. hep-ph/9402266.

[14] G.Farrar and P.Fayet. Phenomenology of the production, decay, and detection of new hadronic states associated with supersymmetry. Phys.Lett., B76:575, 1978.

[15] M.S. Turner and J.A. Tyson. Rev. Mod. Phys., 71(145), 1999. 
[16] L. Girardello and M.T. Grisaru. Soft Breaking of Supersymmetry. Nucl.Phys., B194:65, 1982.

[17] R.N.Mohapatra. Unification and Supersymmetry. Springer-Verlag New York Inc., 2002.

[18] J. Ellis. Beyond the Standard Model for Hillwalkers. Presented at 1998 European School of High-Energy Physics, St. Andrews, Scotland, 23 Aug - 5 Sep 1998., 1998.

[19] J. Lykken L. Hall and S. Weinberg. Supergravity as the Messenger of Supersymmetry Breaking. Phys. Rev., D27:2359, 1983.

[20] S. Chen et. al. Phys.Rev.Lett., 87:251807, 2001. hep-ex/0108032.

[21] LEP2 SUSY Working Group. Combined LEP Chargino Results. http://lepsusy.web.cern.ch/lepsusy/www/inos_moriond01/charginos_pub.html, last update February 2001.

[22] LEP2 SUSY Working Group. Combined LEP Selectron/Smuon/Stau Results. http://lepsusy.web.cern.ch/lepsusy/www/sleptons_summer02/slep_2002.html, last update June 2002.

[23] M. Battaglia et.al. Updated Post-WMAP Benchmarks for Supersymmetry. Eur.Phys.J., C33:273, 2004. hep-ph/0306219.

[24] J.L. Feng. Supersymmetry and cosmology. Annals Phys., 315:2, 2005.

[25] K.A. Olive. TASI Lectures on Dark Matter. Lectures given at the Theoretical Advanced Study Institute in Elementary Particle Physics at the University of Colorado at Boulder - June 2-28, 2002, 2003. astro-ph/0301505.

[26] V. Barger, C.E.M. Wagner et al. Report of the SUGRA Working Group for RunII of the Tevatron. FERMILAB-PUB-00-349, 2000. hep-ph/0003154.

[27] W. Beenakker et al. The Production of Charginos/Neutralinos and Sleptons at Hadron Colliders. Phys. Rev. Lett, 83, 1999. hep-ph/9906298.

[28] D. McGinnis. The Engineering of High Energy Particle Accelerators at Fermi National Accelerator Laborartory. Fermilab, 1997.

[29] S. Abachi et al. The DØ upgrade: The Detector and its physics. Fermilab Pub96/357-E, 1996.

[30] V.M. Abazov et al. The Muon System of the Run II DØ Detector. Fermilab-PUB05-034-E, 2005.

[31] DØ Collaboration. The Muon Trigger at DØ. DØ Note 4099 (C.Leonidopoulos), 2003. 
[32] DØ Collaboration. Global Track Finding at Level 3. DØ Note 3808 (D.Whiteson), 2000.

[33] S.Klimenko et al. Fermilab-FN-0741, 2003.

[34] DØ Collaboration. The Updated DØ Luminosity Determination. DØ Note 4328 (Casey et al.), 2004.

[35] T. Sjöstrand et al. High-Energy-Physics Event Generation with PYTHIA 6.1 . Comp. Phys. Comm., 135:238, 2001. LU TP 00-30, hep-ph/0010017.

[36] H.L. Lai et al. Phys. Rev., D55:1280, 1997. hep-ph/9606399.

[37] DØ Run II Simulation of the DØ Collaboration.

http://www-d0.fnal.gov/computing/MonteCarlo/generators/isajet.html, last update April 2003.

[38] SUSY_TOOLS of the DØ Collaboration.

http://www-d0.fnal.gov/computing/montecarlo/generator_tools/susy_tools.html, last update September 2003.

[39] S. Agostinelli et al. GEANT4: A Simulation Toolkit. Nucl.Instr.Meth., NIM A 506:250, 2003.

[40] Simulators of the DØ Collaboration. D0STAR.

http://www-d0.fnal.gov/computing/MonteCarlo/simulation/d0gstar.html, last update May 2004.

[41] Simulators of the DØ Collaboration.

http://www-d0.fnal.gov/computing/MonteCarlo/simulation/d0sim.html, last update May 2001.

[42] J.M. Campbell and R.K. Ellis. Phys.Rev., D 60:113006, 1999. hep-ph/9905386.

[43] N. Kidonakis and R. Vogt. Phys. Rev., D68:114014, 2003.

[44] DØ Run II Simulation of the DØ Collaboration.

http://www-d0.fnal.gov/computing/MonteCarlo/generators/alpgen.html, last update March 2004.

[45] DØ Collaboration. NNLO Cross-Sections for Drell-Yan, $Z$ and $W$ Production using Modern Parton Distribution Functions. DØ Note 4476 (T.Nunnemann), 2004.

[46] R. Hamberg, W.L. van Neerven and T. Matsuura. A complete calculation of the order alpha-s** 2 correction to the drell-yan k factor. Nucl. Phys. B, 359:343, 1991. [Erratum-ibid. B 644 (2002) 403]. 
[47] Common Sample Group for the DØ Collaboration .

http://www-d0.fnal.gov/Run2Physics/cs/skimming/skimming.html, last update May 2005.

[48] DØ Collaboration. Offline run quality database queries. http://d0db.fnal.gov/qualitygrabber/qualQueries.html.

[49] DØ Collaboration. Global Trigger List Description.

http://www-d0.fnal.gov/trigger_meister/private/www/tl_desc/global.html, last update June 2005. Trigger List: global_CMT-12.37.

[50] D $\varnothing$ Collaboration. Search for the Higgs boson in $H \rightarrow W W^{*} \rightarrow \mu \nu \mu \nu$ decays at D $\varnothing$ RunII. DØ Note 4386 (J. Elmsheuser), 2004.

[51] DØ Collaboration. Search for the Associated Chargino-Neutralino Production in the Final States with Two Muons and Additional Lepton. DØ Note 4806 (M.Binder, R.Ströhmer), 2005.

[52] RunII Luminosity for the DØ Collaboration. http://www-d0.fnal.gov/runcoor/RUN/run2_lumi.html, last update May 2005.

[53] DØ Collaboration. http://www-d0.fnal.gov/computing/algorithms/status/p14.html, last update 2004.

[54] Muon Identification group for the $\mathrm{D} \varnothing$ Collaboration. Certification, last update December 2003.

http://www-d0.fnal.gov/phys_id/muon_id/d0_private/muon_id.html.

[55] J. Elmsheuser. Search for the Higgs Boson in $H \rightarrow W W$ Decays at the DØExperiment and Precise Muon Tracking . PhD thesis, Ludwig-MaximiliansUniversität München, 2004.

[56] $\mathrm{D} \varnothing$ Collaboration. Measurement of $\sigma \cdot B r$ for $Z \rightarrow \mu^{+} \mu^{-}$in $p \bar{p}$ Collisions at $\sqrt{s}=$ 1.96 TeV. DØ Note 4689 (E.Nurse), 2005.

[57] G.C. Blazey et al. Run II jet physics. FERMILAB-CONF-00-092-E, hep-ex/0005012, 2000.

[58] Jet Identification group for the D $\varnothing$ Collaboration. Certified jet energy scale v4.2, last update June 2004.

http://www-d0.fnal.gov/phys_id/jes/d0_private/certified/certified.html.

[59] DØ Collaboration. Jet Energy Scale at DØ RunII. DØ Note 4720 (J. Agram et al.).

[60] Jet Identification group for the DØ Collaboration, last update June 2004. http://www-d0.fnal.gov/computing/algorithms/calgo/met/metdoc.html. 
[61] DØ Collaboration. Search for the Associated Production of Chargino and Neutralino in Final States with Two Electrons and an Additional Lepton. DØ Note 4448 (U.Blumenschein, V.Büscher), 2004.

[62] DØ Collaboration. Search for the Associate Chargino-Neutralino Production in the Final States with two Muons and additional Lepton. DØ Note 4482 (M.Binder and R.Ströhmer), 2004.

[63] DØ Collaboration. Search for the Associated Chargino-Neutralino Production in the Final States with Two Muons and Additional Lepton. Conference note, DØ Note 4567 (M.Binder and R.Ströhmer), 2004.

[64] D.J. Lange. The EvtGen particle decay simulation package . Nucl. Instr. Meth., A462:152, 2001.

[65] DØ Collaboration. D0_MESS (D0 Monte Carlo Event Selection System). http://www-clued0.fnal.gov/d0_mess/.

[66] T. Junk. Nucl.Instr. and Meth., A434(435), 1999.

[67] DØ Collaboration. Measurement of the differential z0-boson production crosssection as function of transverse momentum. DØ Note 4660 (B. Tiller, T. Nunnemann), 2004.

[68] DØ Collaboration. Recommandations of the Ad-Hoc Commitee on Limit-Setting Procedures to be Used by DØ in Run II. DØ Note 4629 (V.Buescher et al.), Oct.,2004.

[69] B. Abbott et al. Phys. Rev. Lett., 80:1591, 1998.

[70] F. Abe et al. Phys. Rev. Lett., 80, 1998.

[71] D $\varnothing$ Collaboration. Search for associated production of Charginos and Neutralinos in final states with three leptons. Conference note DØ Note 4738 (M.Binder et al.), 2005.

[72] V.M. Abazov et al. Search for Supersymmetry via Associated Production of Charginos and Neutralinos in Final States with Three Leptons. submitted to Phys. Rev. Lett, 2005. hep-ex/0504032.

[73] DØ Collaboration. Thumbnail: a compact data format. DØ Note 3979 (S. Protopopescu, S. Baffioni, E. Nagy), 2002.

[74] U. Heintz. Proposal for thumbnail contents. http://www-d0.fnal.gov/ serban/thumbnail/, 2000. 
[75] J. Qian. Computing and Software Model.

http://d0server1.fnal.gov/projects/Computing/Web/Meeting/RAC/d0finance_0702_jq.pdf, 2002. 


\section{Acknowledgements}

I'd like to thank Prof.Dr. Dorothee Schaile, who encouraged me to take up this challenge in the research for new physics. I'd like to thank PD.Dr. Raimund Ströhmer for all the fruitful discussions and who encouraged me to go on with the method for the modeling of the multi-jet background. I'd like to thank Dr. Thomas Nunnemann for the fruitful discussions concerning the production of Monte Carlo Simulations, especially multi-jet, and the work he volonteered to do for all trilepton analyses. I'd like to thank Prof.Dr. Otmar Biebel for all the fruitful discussions about physics and Supersymmetry in particular.

I'd like to thank Dr. Ulrike Blumenschein for the fruitful discussions and all the work producing the supersymmetric trilepton Monte Carlo samples and combining the results of the four analysis including mine. I'd like to thank Dr. Johannes Elmsheuser for setting up a software package influencing the analysis software of the experiment including mine and for answering many questions concerning so-called technical details.

I'd like to thank Dr. Tatajna Kennedy, Dr. Frank Fiedler, Dr. Jörg Dubbert and Dr. Fritz Vollmer for the careful review of the first chapters of this dissertation and Dr. John Kennedy for the review of the short but significant chapters. I'd like to thank Philipp Schieferdecker, with whom I shared an office, for the joyful time. Many thanks to Herta Franz and Britta Tiller, all colleagues at LS Schaile and the Rechnergruppe in Garching. Mulţumesc foarte frumos la Attila Varga, cu care am putut sã vorbesc româneşte din cînd în cînd.

Ich danke meinen Eltern Katharina und Michael, meiner Schwester Hannelore, meinem Bruder Michael, meiner Schwägerin Astrid und meinem Schwager Hans für ihre Unterstützung.

Köszönöm szépen to my friend Ágota Szirmai for her support, her patience and for beeing there. Many filacia to my friend Dr. Sofia Chouridou, who encouraged me to start a doctoral thesis in particle physics. Many thanks to my friend Katrin Berger for the fruitful conversations and for her support and many thanks to my friend Dr. Udo Berger for the wonderful flights in that TB-10 airplane and for his support. Many thanks to my dear friend Sander Hos for his support and for beeing there. Many thanks to my friend Ferry van Ruijven, who asked me many questions about the purpose of this dissertation.

"Thank you" to all friends who cheered me up and gave me courage during the last years, especially my friend Julia Buchloh who invited me to Sweden, where I could spent a wonderful christmas in 2004. 



\title{
Curriculum Vitae
}

\author{
Meta Binder \\ LMU Sektion Physik \\ Am Coulombwall 1 \\ 85748 Garching \\ e-mail: meta.binder@physik.uni-muenchen.de
}

\section{Personal Information}

born 19.06.1974 in Sighişoara / România

\section{Education}

1980 - 1984 Şcoala generalã Şaeş / România

1984 - 1988 Şcoala generalã Apold / România

1988 - 1990 Liceu industrial Josheph Haltrich Sighişoara / România

1990 - 1993 Finsterwalder Gymnasium Rosenheim

1993 - 2001 study of physics at the Ludwig-Maximilians-Universität München

Diploma thesis at the OPAL experiment: Search for Single Top Quark Production at LEP2

in the Leptonic W Boson Decay Channel

\section{Doctorate}

since 2001 at Ludwig-Maximilians-Universität München

field of research: new phenomena in elementary particle physics

Dissertation at the DØ experiment: Search for the Trilepton Signal of the Minimal Supergravity Model in DØ Run II

\section{Work experience}

01.07.02 - 31.03.03 guest scientist at the Fermi National Accelerator Laboratory Chicago / Illinois

01.09.02 - 10.12.02 Data Aquisition Shifter at the DØ Experiment

24.08.03 - 06.09.03 Participation at the European Summer School for High-Energy-Physics in Tzakhkadzor / Armenia

27.06.04 - 02.07.04 Participation at the annual meeting of the Nobelpricewinners in Lindau

\section{Memberships}

since 1995 Deutsche Physikalische Gesellschaft

since 2001 DØ Collaboration

since 2004 Graduiertenkolleg Particle Physics at the Energy Frontier of New Phenomena

\section{Publications}

V. M. Abazov et al., Search for Supersymmetry via Associated Production of Charginos and Neutralinos in Final States with Three Leptons, submitted to Phys. Rev. Lett, 2005, hep-ex/0504032 
